\title{
WestVirginiaUniversity
}

THE RESEARCH REPOSITORY @ WVU

Graduate Theses, Dissertations, and Problem Reports

2014

\section{Polylactic Acid-Based Polymer Blends for Durable Applications}

\author{
Adam Finniss \\ West Virginia University
}

Follow this and additional works at: https://researchrepository.wvu.edu/etd

\section{Recommended Citation}

Finniss, Adam, "Polylactic Acid-Based Polymer Blends for Durable Applications" (2014). Graduate Theses, Dissertations, and Problem Reports. 596.

https://researchrepository.wvu.edu/etd/596

This Dissertation is protected by copyright and/or related rights. It has been brought to you by the The Research Repository @ WVU with permission from the rights-holder(s). You are free to use this Dissertation in any way that is permitted by the copyright and related rights legislation that applies to your use. For other uses you must obtain permission from the rights-holder(s) directly, unless additional rights are indicated by a Creative Commons license in the record and/ or on the work itself. This Dissertation has been accepted for inclusion in WVU Graduate Theses, Dissertations, and Problem Reports collection by an authorized administrator of The Research Repository @ WVU.

For more information, please contact researchrepository@mail.wvu.edu. 
Polylactic Acid-Based Polymer Blends for Durable Applications

Adam Finniss

Dissertation submitted to the

Benjamin M. Statler College of Engineering and Mineral Resources

at West Virginia University

in partial fulfillment of the requirements

for the degree of

Doctor of Philosophy

in

Chemical Engineering

Rakesh K. Gupta, Ph.D., Chair

Sushant Agarwal, Ph.D.

Robin Hissam, Ph.D.

Edward M. Sabolsky, Ph.D.

X.Y. Li, Ph.D.

Department of Chemical Engineering

Morgantown, West Virginia

2014

Keywords: Biodegradable Polymers, Polymer Blends, Polymer Stability

Copyright 2014 Adam Finniss 


\section{ABSTRACT \\ Polylactic Acid-Based Polymer Blends for Durable Applications}

Adam Finniss

There has been considerable scientific interest in both research and commercial communities as of late in the area of biologically based or sourced plastics. As the consumption of petroleum rises and concerns about climate change increase, this field is likely to grow even larger. One bioplastic that has received a great deal of attention is polylactic acid (PLA). In the past, this material was used mainly in medical or specialty applications, but advancements in manufacturing have led to a desire to use PLA more widely, especially in durable applications. Unfortunately, PLA has several drawbacks that hinder more widespread usage of the material as a durable item: it has low ductility and impact strength in bulk applications, along with poor stability in the face of heat, humidity or liquid media. To combat these deficiencies, a number of techniques were investigated. Samples were annealed to create crystalline domains that would improve mechanical properties and reduce diffusion, blended with graphene to create barriers to diffusion throughout the material, or compounded with a polycarbonate (PC) polymer phase to protect the PLA phase and to enhance the mechanical properties of the blend. If a material containing biologically sourced components with good mechanical properties can be created, it would be desirable for durable uses such as electronics components or as an automotive grade resin.

Crystallization experiments were carried out in a differential scanning calorimeter to determine the effects of heat treatment and additives on the rather slow crystallization kinetics of PLA polymer. It was determined that the blending in of the PC phase did not significantly alter the kinetics or mechanism of crystal growth. The addition of graphene to any PC/PLA formulation served as a nucleating agent which speeded up the crystallization kinetics markedly, in some cases by several orders of magnitude. Results obtained from these experiments were internally consistent, showing that no matter the treatment or formulation, PLA achieved a maximum of 30-35 percent crystallinity.

Samples receiving no treatment as well as those with annealing, the addition of graphene, and in some cases annealing/graphene were subjected to both solvent and hydrolytic degradation in order to find the most stable blend or treatment. Both pellets and molded parts of varying thicknesses were investigated to evaluate the effect of diffusional resistance on long term durability. It was determined that while the addition of crystallinity or graphene platelets can provide a temporary barrier against diffusion of attacking species, PLA polymer itself is not dimensionally stable over the long lifecycle required for durable applications such as for automotive parts. In fact, PLA-only molded panels aged in distilled water at $50^{\circ} \mathrm{C}$ for 42 days experienced over $99 \%$ viscosity loss regardless of which treatment was applied, and nearly all 
mechanical strength was lost during this time. Furthermore, while the addition of graphene and the heat treatment produced diffusion barriers which could slightly enhance PLA's degradation resistance, the treatments caused the already fragile polymer to become very brittle. Solvent degradation experiments also showed that molded parts containing more than $40 \%$ PLA loading lost in excess of $75 \%$ of the original viscosity no matter what treatment was used. This showed that these materials are likely to fail well before a sufficiently long lifecycle for durable goods is achieved.

Polycarbonate rich blends with less than 30\% PLA as the dispersed phase showed excellent property retention after the accelerated aging tests. Formulations with up to $20 \%$ PLA content had degradation results that were nearly identical to those of $100 \%$ polycarbonate, which literature has shown to have useful lifecycles for durable applications of up to 20 years. By completely encapsulating the PLA in the polycarbonate matrix, which occurred at about $30 \%$ PLA by maximum, it was fully protected by the more stable phase. An investigation was undertaken to alter the morphology of the encapsulated PLA droplets by virtue of different mixing conditions; particle sizes ranging from about 1-6 microns could be created by changing the processing steps. However, no great increase in degradation resistance was observed by minimizing the interfacial surface area. It seemed likely that the effects of shear and thermal history experienced during the compounding step played a larger role in the durability of these formulations.

Lastly, molded parts of differing thicknesses were hydrolytically degraded to examine the effects of diffusion resistance on the mechanical properties of untreated PC/PLA blends. It was determined that, similar to the droplet morphology study, the effect of PC content was the most dominating factor in the durability of the formulations. In fact, if molded parts reach a critical thickness, a transition from ductile to brittle failure modes can be observed. The rate of diffusion through the materials was also determined to be much faster than the rate of PLA hydrolysis.

It is concluded that the most effective way to create a durable material containing a significant bio-based content is to completely encapsulate PLA polymer with the more stable polycarbonate phase. Materials containing up to about 30\% PLA at maximum were shown to be sufficiently durable so that they may be employed in similar automotive and electrical applications as for pure polycarbonate. 


\section{ACKNOWLEDGEMENT}

I would like to thank Dr. Gupta for extending the generous offer of the Bayer Fellowship to complete my doctorate. He has also been my mentor throughout graduate school and I appreciate his knowledge and guidance. Dr. Sushant Agarwal has been of great help to me while performing the experiments required for this work and for teaching me how to use new instruments and techniques when necessary. I also thank Dr. Robin Hissam and Dr. Edward Sabolsky for their advice and knowledge, especially in the field of material science and engineering. Dr. XY Li of Bayer MaterialScience has also provided great insight into my project, and I am thankful for his support and that of many others at Bayer (Dr. Marina Rogunova, Marie Urick, and Diana Mullen among them) while on an internship there.

Thanks also to my friends and colleagues who helped me out in and out of the lab: Matthew Thompson, Elliot Roth, Chris Yurchick, Jeremy Hardinger and many others.

Thanks to the Bayer Foundation for funding several Ph.D. students through a generous donation. 


\section{TABLE OF CONTENTS}

1 INTRODUCTION 1

1.1 Biologically Derived Chemicals and Plastics. .............................................................. 1

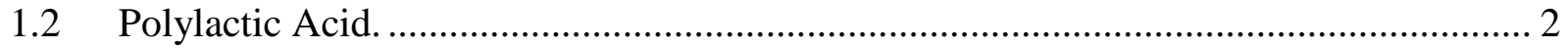

1.2.1 Modification of the Polylactic Acid Component................................................... 4

1.3 Blends of Polylactic Acid and Polycarbonate ................................................................ 6

1.4 Objectives of the Current Work. ................................................................................. 8

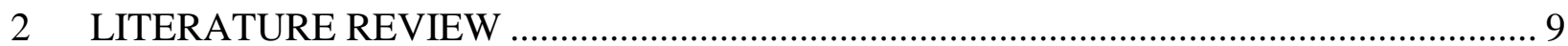

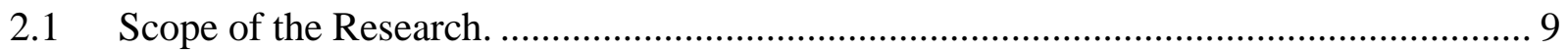

2.2 Degradation of PLA and PLA-Based Materials...................................................... 9

2.3 Degradation Behavior of Polycarbonate ................................................................. 20

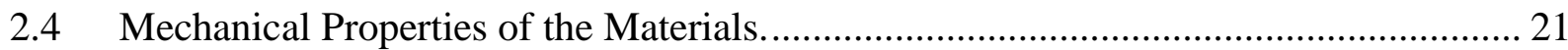

2.4.1 Mechanical Properties of PLA-Based Blended Materials....................................... 21

2.5 Crystalline/Thermal Properties of Polymeric Materials................................................. 26

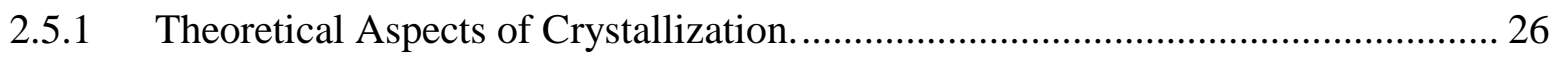

2.5.2 Crystallization of PLA-Based Materials............................................................... 31

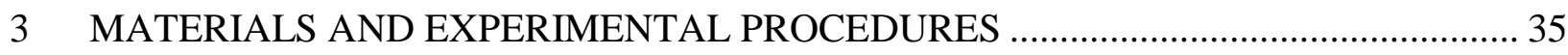

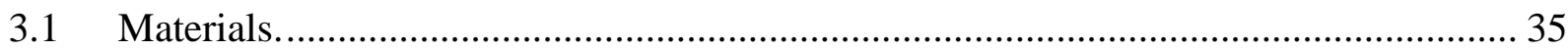

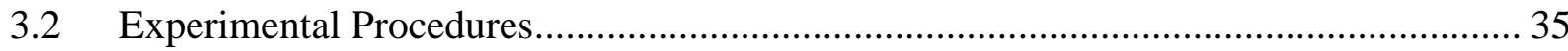

3.2.1 Compounding of the Polycarbonate and Polylactic Acid......................................... 35

3.2.2 Compounding of the Polycarbonate and Polylactic Acid with Graphene. ............. 37

3.2.3 Compression Molding.................................................................................. 38

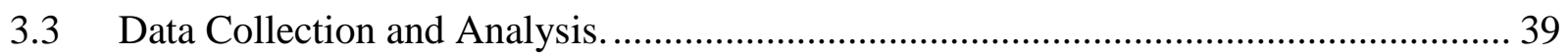

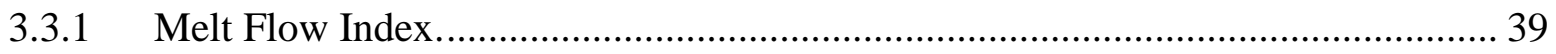

3.3.2 Mechanical Testing..................................................................................... 40

3.3.3 Differential Scanning Calorimetry................................................................. 41

3.3.4 Parallel Plate Rheology................................................................................... 42

3.3.5 Degradation in Acid/Base Media....................................................................... 42

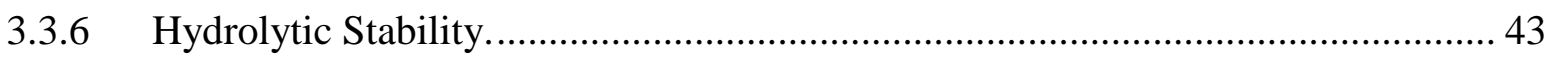

3.3.7 Morphological Studies................................................................................. 43

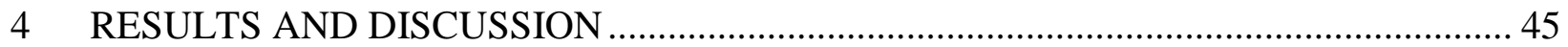




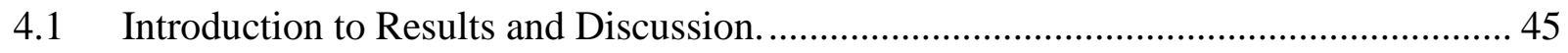

4.2 Effect of Untreated vs. Treatments of PLA Polymer................................................... 45

4.2.1 Crystallization of the Extruded PLA and PLA/Graphene Composite..................... 47

4.2.2 Effect of Treatment on Initial Crystallinity of PLA and PLA/Graphene Composite. 54

4.2.3 Solvent Degradation of PLA and PLA/Graphene Composites............................... 55

4.2.4 Hydrolytic Degradation of PLA and PLA/Graphene Composites.......................... 65

4.3 Gel Permeation Chromatography (GPC) Results for PLA-only Materials.................... 72

4.4 Commentary on PLA and PLA/Graphene Durability.................................................... 74

4.5 Blends of Polylactic Acid and Polycarbonate............................................................. 74

4.5.1 Fabrication of the PC/PLA and PC/PLA/Graphene Composites............................ 74

4.5.2 Crystallization of the PC/PLA and PC/PLA/Graphene Composites. ....................... 79

4.5.3 Initial Crystallinity of PC/PLA and PC/PLA/Graphene Composites. ..................... 81

4.5.4 Solvent Degradation of PC/PLA and PC/PLA/Graphene Composites.................... 83

4.5.5 Hydrolytic Degradation of PC/PLA and PLA/Graphene Composites..................... 91

4.6 Commentary on PC/PLA and PC/PLA/Graphene Durability..................................... 100

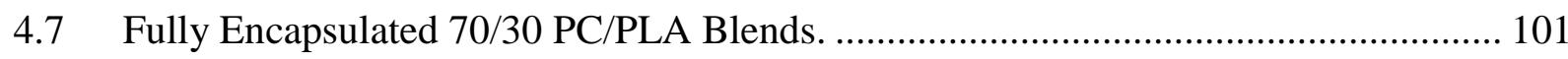

4.7.1 Solvent Degradation of Fully Encapsulated PC/PLA Blends............................... 103

4.7.2 Hydrolytic Degradation of Fully Encapsulated PC/PLA Blends.......................... 108

4.8 Commentary on Fully Encapsulated 70/30 PC/PLA Blend Durability......................... 112

4.9 Effect of Specimen Thickness on PC/PLA Blend Durability ...................................... 113

4.9.1 Comparison of Thin-Walled and Thick-Walled Flexural Bars............................. 115

5 CONCLUSIONS AND RECOMMENDATIONS. ......................................................... 120

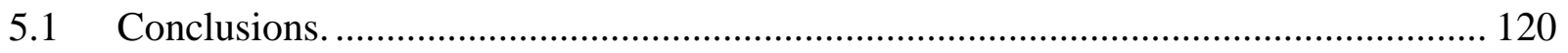

5.2 Recommended or Plausible Further Studies. .......................................................... 122

Appendix A: Viscosity Curves for Solvent Degradation........................................................... 133 


\section{LIST OF FIGURES}

Figure 1.1: Projection for bio-based plastic growth by $2020^{4}$................................................. 1

Figure 1.2: Projection for world bioplastic growth by $2016^{5}$.................................................... 2

Figure 1.3: Chirality of lactic acid and lactide give rise to chirality of PLA polymer ${ }^{16} \ldots \ldots \ldots \ldots \ldots \ldots . . .4$

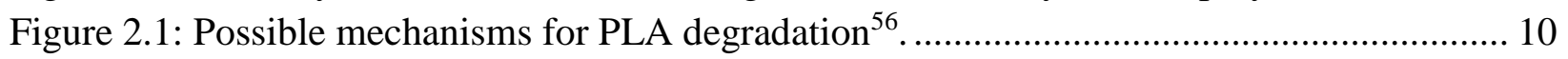

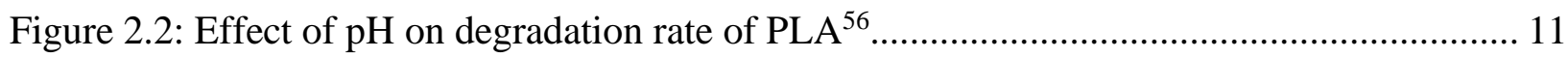

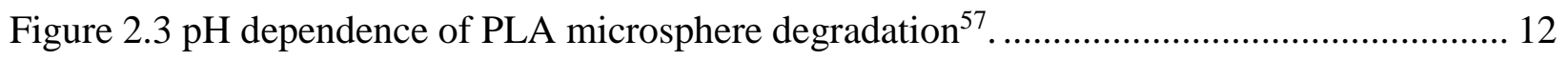

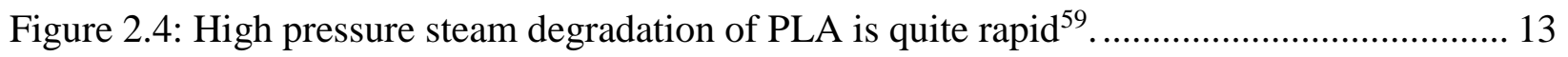

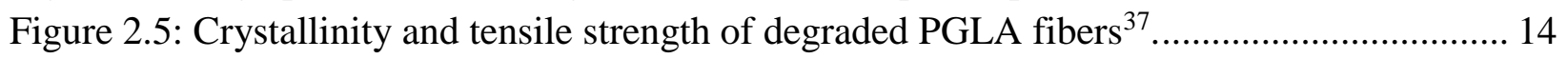

Figure 2.6: Surface or bulk erosion may take place for a given material ${ }^{60}$. ............................... 15

Figure 2.7: Thermal properties change with molecular orientation ${ }^{18}$......................................... 16

Figure 2.8: Changes in mechanical properties after boiling water exposure ${ }^{18}$........................... 17

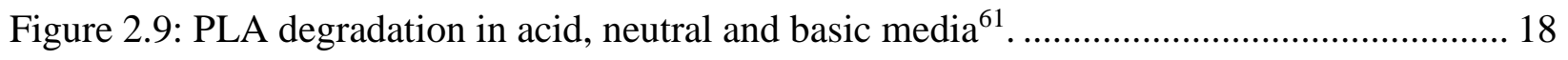

Figure 2.10: Addition of clay reduces $\mathrm{H}_{2} \mathrm{O}$ transmission of PLA films ${ }^{68}$................................. 19

Figure 2.11: Hydrolysis of $\mathrm{PC}$ in a $300^{\circ} \mathrm{C}$ water/steam bomb reactor ${ }^{41}$.................................... 20

Figure 2.12: Compatibilization of PLLA/ABS blends enhances properties ${ }^{28}$............................. 23

Figure 2.13: Inappropriate loading of clay causes properties to decrease ${ }^{68}$................................ 25

Figure 2.14: Loss of properties due to aggregation of nano-scale fillers ${ }^{79}$.................................. 25

Figure 2.15: Contribution of energy terms in generation of possible crystal growth nuclei ${ }^{80} \ldots . .27$

Figure 2.16: General shape of transformation curve on time dependence ${ }^{81}$................................ 29

Figure 2.17: Curve fit of kinetic parameter for various PLA specimens ${ }^{85}$................................. 31

Figure 2.18: PLA crystallinity and blending affect miscibility of PLA/PMMA blends ${ }^{74}$............ 33

Figure 3.1: Leistritz 27 TSE with feeders, control box and vacuum trunk.................................. 36

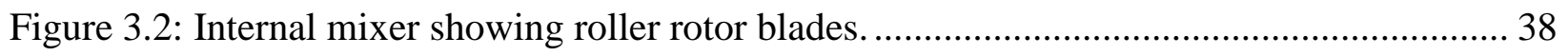

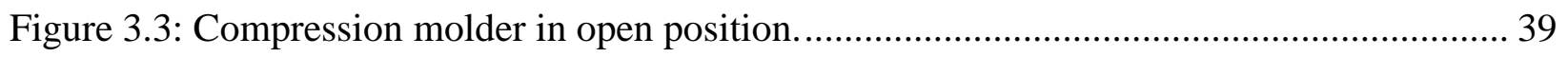

Figure 3.4: Melt flow indexer with piston and weight during test.............................................. 40

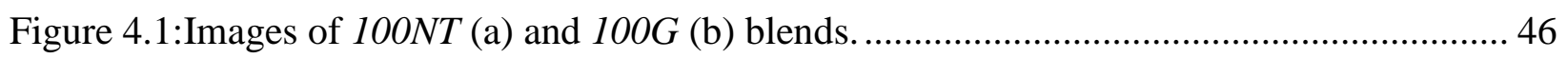

Figure 4.2: Images of amorphous (a) 100\% PLA square contrasted with annealed (b) 100\% PLA

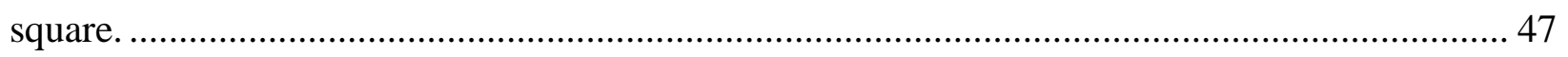

Figure 4.3: Heat flow from sample during isothermal crystallization experiment. ...................... 48

Figure 4.4: Time-transformation curve for the data presented in Figure 4.3 ............................... 49

Figure 4.5: Avrami plot of previous sample over 5-20\% conversion range................................ 50

Figure 4.6: Avrami exponent for primary crystallization of $100 \mathrm{~A}$ and $100 \mathrm{G}$ blends................... 51

Figure 4.7: Kinetic parameter for primary crystallization of $100 \mathrm{~A}$ and $100 \mathrm{G}$ blends. ................. 51

Figure 4.8: Crystallization on cooling for sample containing 98/2 PLA/graphene...................... 53

Figure 4.9: Initial crystallinity of 98-100\% PLA parts and pellets by treatment option. .............. 55

Figure 4.10: Weight loss of PLA and PLA/graphene samples during exposure to acetic acid

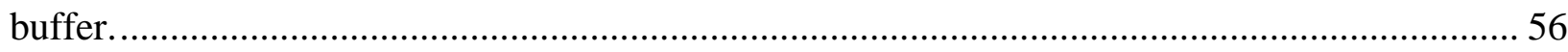

Figure 4.11: Weight loss of PLA and PLA/graphene samples during exposure to distilled water. 
Figure 4.12: Weight loss of PLA and PLA/graphene samples during exposure to carbonate buffer.

Figure 4.13: Development of crystallinity in PLA and PLA/graphene during exposure to acetic acid buffer.

Figure 4.14: Development of crystallinity in PLA and PLA/graphene during exposure to distilled water. 59

Figure 4.15: Development of crystallinity in PLA and PLA/graphene during exposure to carbonate buffer. 59

Figure 4.16: Viscosity change of untreated PLA after exposure to acetic acid buffer............... 61

Figure 4.17: Viscosity change of untreated PLA after exposure to distilled water.................... 61

Figure 4.18: Viscosity change of untreated PLA after exposure to carbonate buffer.................. 62

Figure 4.19: Viscosity change of annealed PLA after exposure to distilled water..................... 63

Figure 4.20: Viscosity change of PLA/graphene after exposure to distilled water. ................... 63

Figure 4.21: Comparison of viscosity loss between varying PLA treatments........................... 64

Figure 4.22: Metal rack for hydrolytic aging of flexural bars. ............................................ 65

Figure 4.23: Initial flexural modulus of untreated, annealed and PLA/graphene materials........ 66

Figure 4.24: Initial flexural strength of untreated, annealed and PLA/graphene materials. ......... 67

Figure 4.25 Initial flexural ductility of untreated, annealed and PLA/graphene materials.......... 67

Figure 4.26: Flexural modulus of untreated, annealed and PLA/graphene flexural bars after

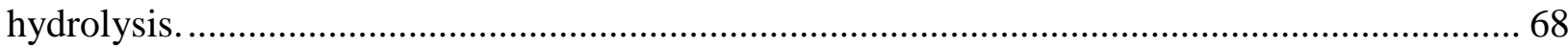

Figure 4.27: Flexural strength of untreated, annealed and PLA/graphene flexural bars after

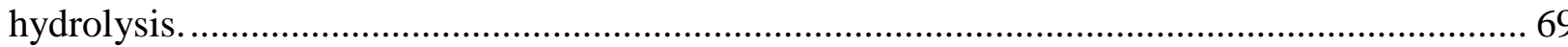

Figure 4.28: Flexural ductility of untreated, annealed and PLA/graphene flexural bars after hydrolysis.

Figure 4.29: Increase in MFI of untreated, annealed, and PLA/graphene materials after hydrolysis. 71

Figure 4.30: Normalized change in MFI for untreated, annealed, and PLA/graphene materials

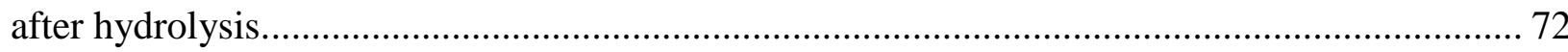

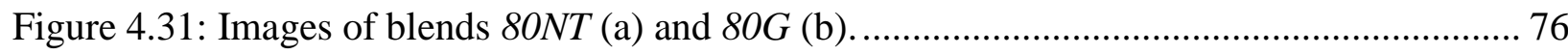

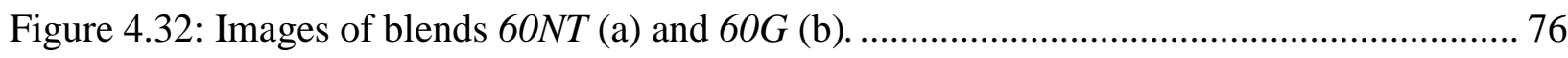

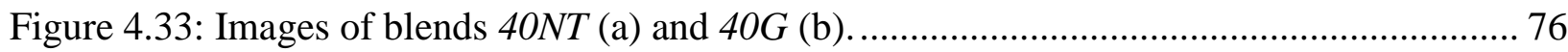

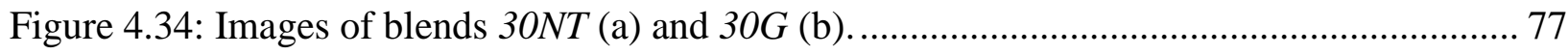

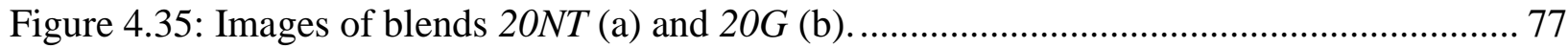

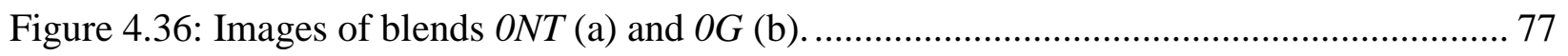

Figure 4.37: Avrami exponent for primary crystallization of PC/PLA and PC/PLA/graphene

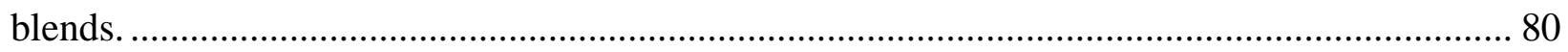

Figure 4.38: Kinetic parameter for primary crystallization of PC/PLA and PC/PLA/graphene

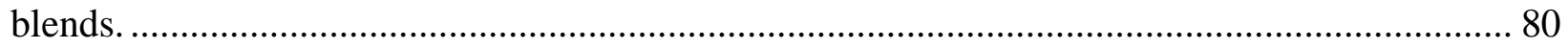

Figure 4.39: Initial crystallinity of PC/PLA and PC/PLA/graphene pellet samples................... 82

Figure 4.40: Initial crystallinity of PC/PLA and PC/PLA/graphene molded parts..................... 82 
Figure 4.41: Weight loss of untreated PC/PLA molded panels during exposure to carbonate

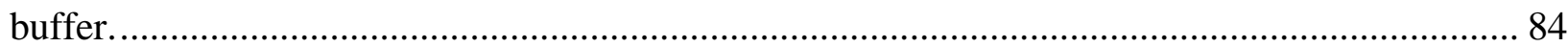

Figure 4.42: Viscosity change of untreated $80 \%$ PLA panels after exposure to distilled water... 85 Figure 4.43: Viscosity change of untreated $80 \%$ PLA panels after exposure to distilled water... 85 Figure 4.44: Viscosity change of 79\% PLA/graphene panels after exposure to distilled water... 86 Figure 4.45: Comparison of viscosity loss between 79-80\% PLA blends with varying treatments.

Figure 4.46: Comparison of viscosity loss between 59-60\% PLA blends with varying treatments. 88

Figure 4.47: Comparison of viscosity loss between 39-40\% PLA blends with varying treatments. 88

Figure 4.48: Comparison of viscosity loss between 29-30\% PLA blends with varying treatments. 89

Figure 4.49: Comparison of viscosity loss between 19-20\% PLA blends with varying treatments.

Figure 4.50 Comparison of viscosity loss between 0\% PLA blends with varying treatments..... 90 Figure 4.51: Initial flexural modulus of the PC/PLA and PC/PLA/graphene materials with

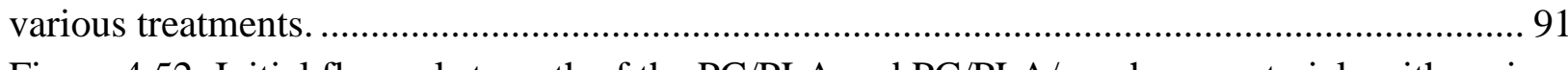
Figure 4.52: Initial flexural strength of the PC/PLA and PC/PLA/graphene materials with various treatments. 92

Figure 4.53: Initial flexural ductility of the PC/PLA and PC/PLA/graphene materials with

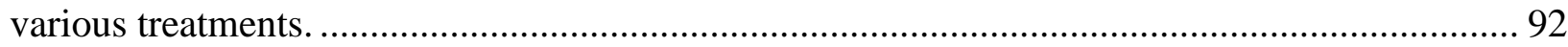

Figure 4.54: Flexural strength loss of untreated PC/PLA bars after hydrolysis. ....................... 93

Figure 4.55: Flexural strength loss of annealed PC/PLA bars after hydrolysis......................... 94

Figure 4.56: Flexural strength loss of PC/PLA/graphene bars after hydrolysis. ........................ 94

Figure 4.57: Flexural strength loss of annealed PC/PLA/graphene bars after hydrolysis........... 95

Figure 4.58: Increase in MFI of untreated PC/PLA pellets after hydrolysis. ............................ 96

Figure 4.59: Increase in MFI of annealed PC/PLA pellets after hydrolysis............................. 96

Figure 4.60: Increase in MFI of PC/PLA/graphene pellets after hydrolysis. ............................ 97

Figure 4.61: Increase in MFI of annealed PC/PLA/graphene pellets after hydrolysis. ............... 97

Figure 4.62: Normalized change in MFI for untreated PC/PLA pellets................................... 98

Figure 4.63: Normalized change in MFI for annealed PC/PLA pellets................................... 99

Figure 4.64: Normalized change in MFI for PC/PLA/graphene pellets. ................................... 99

Figure 4.65: Normalized change in MFI for annealed PC/PLA/graphene pellets.................... 100

Figure 4.66: Images of internally mixed 70/30 PC/PLA blends 30B-E. .............................. 102

Figure 4.67: Viscosity change of sample 30NT after distilled water degradation..................... 104

Figure 4.68: Viscosity change of sample 30B after distilled water degradation. ...................... 104

Figure 4.69: Viscosity change of sample 30C after distilled water degradation...................... 105

Figure 4.70: Viscosity change of sample 30D after distilled water degradation...................... 105

Figure 4.71: Viscosity change of sample 30E after distilled water degradation. ..................... 106 
Figure 4.72: Comparison of viscosity loss between 30\% PLA blends of different internal

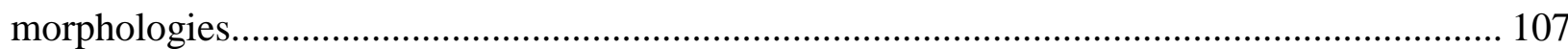

Figure 4.73: Initial flexural modulus of the encapsulated 70/30 PC/PLA blends. ................... 108

Figure 4.74: Initial flexural strength of the encapsulated 70/30 PC/PLA blends..................... 109

Figure 4.75: Initial flexural ductility of the encapsulated 70/30 PC/PLA blends..................... 109

Figure 4.76: Flexural strength loss of encapsulated 70/30 PC/PLA blends of different internal

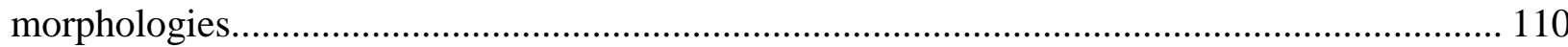

Figure 4.77: Increase in MFI of extruded and internally mixed encapsulated 70/30 PC/PLA

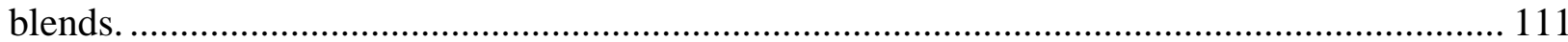

Figure 4.78: Normalized change in MFI for extruded and internally mixed encapsulated 70/30

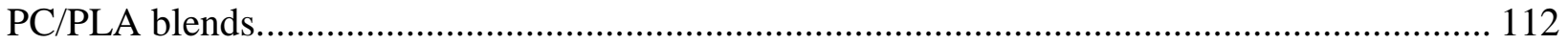

Figure 4.79: Representation of flexural bar exposed to humidity chamber............................. 114

Figure 4.80: Initial flexural moduli of the thin and thick molded untreated PC/PLA blends. ... 116

Figure 4.81: Initial flexural strength of the thin and thick molded untreated PC/PLA blends... 116

Figure 4.82: Initial flexural ductility of the thin and thick molded untreated PC/PLA blends... 117

Figure 4.83: Flexural strength loss of thin and thick molded untreated PC/PLA blends. ......... 118

Figure 4.84: Flexural ductility loss of thin and thick molded untreated PC/PLA blends.......... 118

Figure A 1: Viscosity change of sample 60NT after distilled water degradation...................... 133

Figure A 2: Viscosity change of sample 60A after distilled water degradation ....................... 133

Figure A 3: Viscosity change of sample 60G after distilled water degradation....................... 134

Figure A 4: Viscosity change of sample 40NT after distilled water degradation..................... 134

Figure A 5: Viscosity change of sample 40A after distilled water degradation ....................... 135

Figure A 6: Viscosity change of sample 40G after distilled water degradation ....................... 135

Figure A 7: Viscosity change of sample 40AG after distilled water degradation ..................... 136

Figure A 8: Viscosity change of sample 30A after distilled water degradation ....................... 136

Figure A 9: Viscosity change of sample 30G after distilled water degradation ...................... 137

Figure A 10: Viscosity change of sample 30AG after distilled water degradation ................... 137

Figure A 11: Viscosity change of sample 20NT after distilled water degradation.................... 138

Figure A 12: Viscosity change of sample 20A after distilled water degradation ...................... 138

Figure A 13: Viscosity change of sample 20G after distilled water degradation ...................... 139

Figure A 14: Viscosity change of sample 20AG after distilled water degradation ................... 139

Figure A 15: Viscosity change of sample 0NT after distilled water degradation..................... 140

Figure A 16: Viscosity change of sample 0G after distilled water degradation ....................... 140 


\section{LIST OF TABLES}

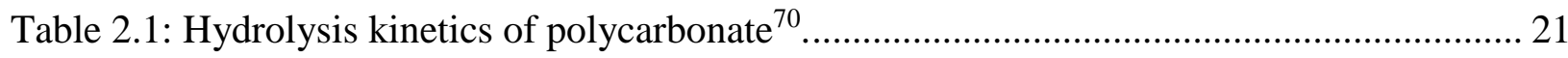

Table 2.2: Dimensional estimates for crystalline patterns ${ }^{81}$............................................... 30

Table 2.3: Crystallization experiments with PLA/PBAT blends at $128^{\circ} \mathrm{C}^{30}$............................ 32

Table 2.4: Composition and process dependent crystallization of PET/PLA blend ${ }^{34}$................ 33

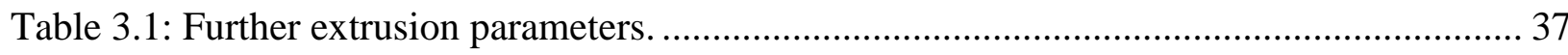

Table 3.2: Solution conditions and times for degradation tests.......................................... 42

Table 4.1 PLA experimental materials and method of production......................................... 46

Table 4.2: GPC results of various PLA and PLA/graphene samples. ....................................... 73

Table 4.3: PC/PLA and PC/PLA/Graphene experimental materials and method of production.. 75

Table 4.4: Particle size analysis of PC/PLA and PC/PLA/graphene composites...................... 78

Table 4.5: Viscosity loss at $1 \mathrm{rad} / \mathrm{s}$ after 42 days aging for the PC/PLA and PC/PLA/graphene

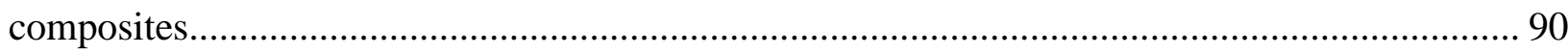

Table 4.6: Mixing conditions for encapsulated 70/30 PC/PLA blends. .................................. 101

Table 4.7: Particle sizes for the 70/30 PC/PLA blends......................................................... 102

Table 4.8: Theoretical interfacial surface areas of internally mixed 70/30 blends with respect to extruded blend. 103

Table 4.9: Viscosity loss at $1 \mathrm{rad} / \mathrm{s}$ after 42 days aging for the $30 \%$ PLA blends of different internal morphologies. 


\section{CHAPTER 1}

\section{INTRODUCTION}

\subsection{Biologically Derived Chemicals and Plastics.}

Biologically based plastics have experienced considerable research and commercial interest in recent years. Trends such as ever-increasing global consumption of petroleum for energy and transportation, coupled with concerns about anthropogenic climate change, as well as a growing "green" movement both domestically and abroad should see this area expand additionally in the short to medium term. Therefore, a variety of biologically derived plastics such as polyhydroxyalkanoate (PHA), polytrimethylene terephthalate (PTT), polylactic acid (PLA), as well as starch and cellulosic polymers have become popular in industry and the literature (for example ${ }^{1,2,3}$ ). In fact, a 2009 technical report suggested the 2007 global budget of 360,000 tons of annual bioplastic production could increase to up to 2.3 million tons in 2013, an increase of $37 \%$ per annum ${ }^{4}$. While ongoing economic forces and the explosive growth of shale gas production for feedstocks may alter these forecasts, these authors predicted fairly stable growth, as shown in Figure 1.1:

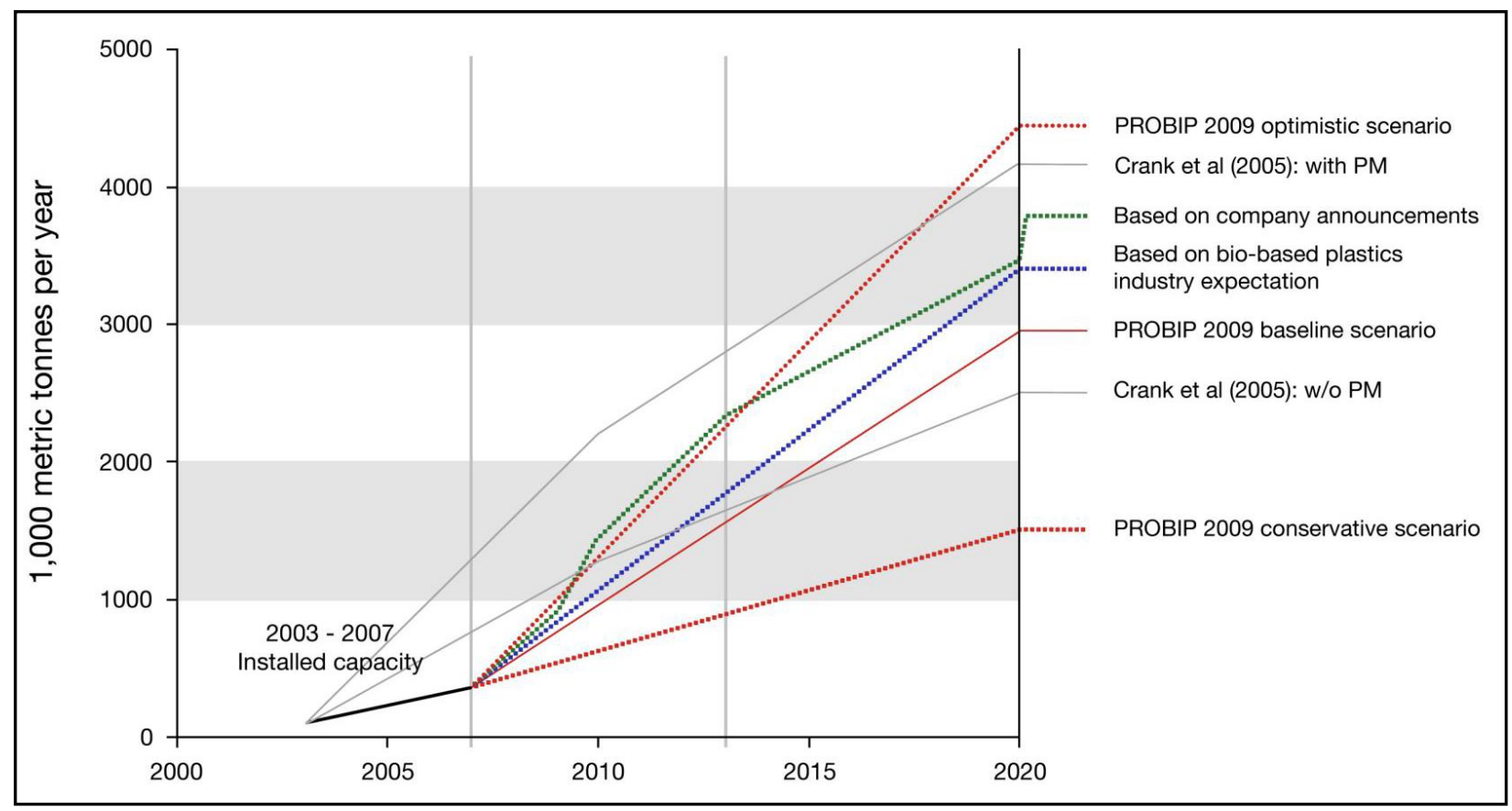

Figure 1.1: Projection for bio-based plastic growth by $2020^{4}$. 
Recent reports have also suggested that this growth is continuing to expand, especially into rapidly growing economies such as those in South America and Asia, as shown in Figure 1.25:

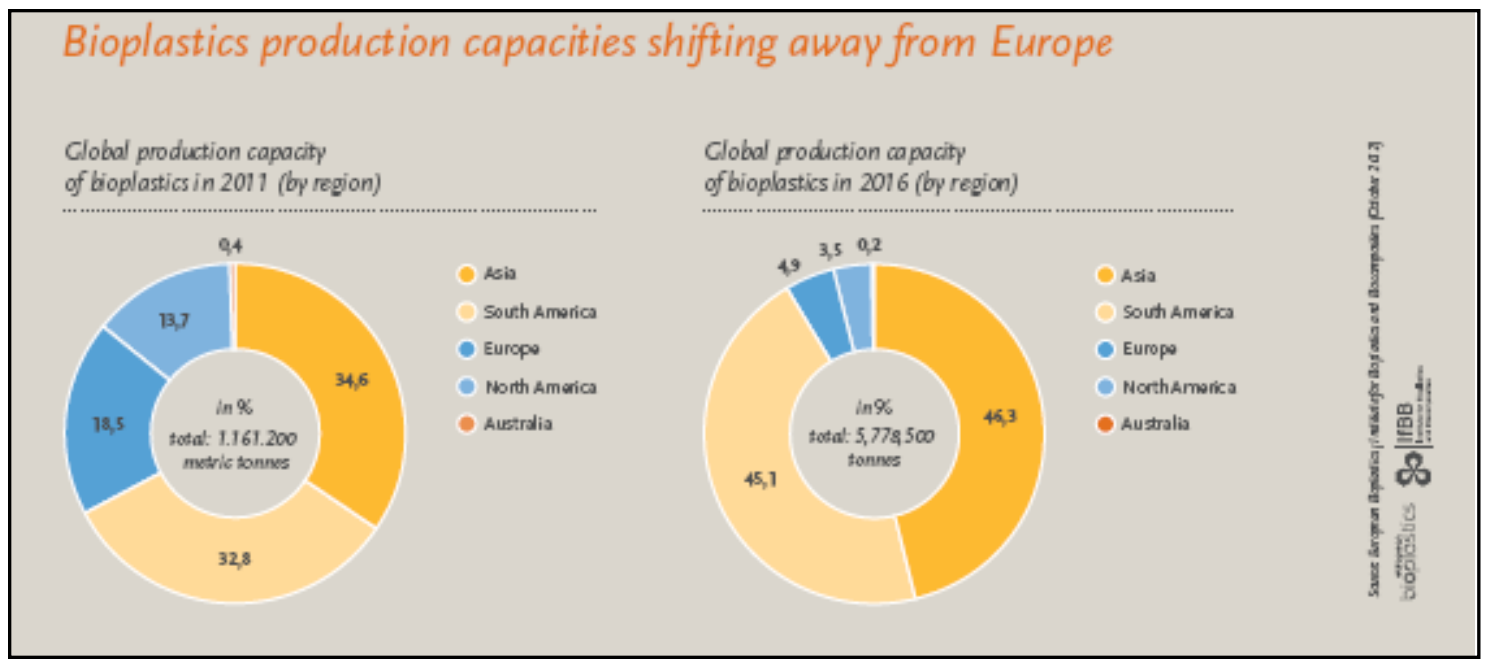

Figure 1.2: Projection for world bioplastic growth by $2016^{5}$.

In the United States, there are significant inroads to be had for naturally sourced feedstocks. As of 2004, the U.S. budget for organic chemicals, lubricants, and plastics was nearly 90 million tonnes, the majority of which is conventional but could be augmented or replaced with green sources $^{6}$. This is not to suggest that swapping agriculturally derived plastics in for the 50 million tonnes of petroleum based ones produced yearly is a trivial undertaking. For instance, some polymers such as polyether ether ketone (PEEK) have tremendous high temperature properties that simply cannot be matched by any natural organic materials. Thus, target areas where biopolymers can successfully supplant conventional plastics would likely be for non-extreme condition applications such as packaging and fiber applications ${ }^{6}$. Here, polylactic acid (PLA) has been shown to be an attractive plastic option that combines favorable properties of both natural fiber and synthetic polymer ${ }^{6}$. PLA is a versatile semi-crystalline material that has been the subject of especially fervent interest as of late, and that shall likely continue into the future if the predictions of European Bioplastics and other pro-industry groups come to fruition.

\subsection{Polylactic Acid.}

As far back as 1932, Wallace Carothers and his team were able to create low molecular weight PLA at DuPont's Experimental Station ${ }^{7}$. For most of the $20^{\text {th }}$ century, however, widespread 
usage was both economically and technically unfeasible ${ }^{7,8}$. The high cost of production and the primitive fermentation science available limited deployment of PLA to mostly the medical realm; it was commonly used for sutures, implants, and drug delivery systems. Then, by around 1990, a global research focus emerged on commoditizing PLA, with the Americans, Europeans and Japanese taking the lead ${ }^{1,6,8}$. At present, companies like the American NatureWorks (the remnant of a Cargill-Dow joint venture) and Purac in the Netherlands are leading manufacturers. The material is still used biomedically, but has further been introduced into food and packaging roles due to its in vivo compatibility (e.g. ${ }^{9}$ ). Current production is based on fermentation of a variety of biomass sugars, but expected future technological advances would allow waste products such as corn stover and cellulosic husks to serve as raw materials as well ${ }^{1,6}$. This would obviously improve the overall efficiency in utilizing materials throughout their agricultural lifecycle, and likely serve to stabilize price uncertainties due to fluctuating fresh crop availability. Ecologically, production of a unit of PLA has been estimated to save up to $50 \%$ in fossil resources and reduce GHG emissions over its lifecycle by $70 \%$ than a unit of competitive products like poly(ethylene terephthalate) (PET) or Nylon ${ }^{1,7,8,10}$. However, there are still drawbacks in introducing these natural materials as wholesale replacements for conventional plastics. PLA, in fact, has the following flaws that must be addressed in order to increase its usage in durable applications:

- Low glass transition temperature and low thermal stability that prevent high temperature applications (e.g. ${ }^{1,9}$ ).

- Rapid physical aging leads to brittleness for bulk PLA applications at room temperature. The period over which significant physical aging occurs has been reported to be as little as 3 to 8 hours $^{11}$ !

- High susceptibility to degradation by attacking species or ions, such as acid and base (e.g. $\left.{ }^{12}\right)$.

- Very poor impact strength, as noted by the manufacturer ${ }^{13}$.

For these reasons, polylactic acid itself has been subject to a number of treatments in attempts to minimize those shortcomings. 


\subsubsection{Modification of the Polylactic Acid Component.}

The most simplistic technique for improving PLA properties that researchers have reported is enhancing the PLA crystallinity so that the mechanical properties and heat resistance are improved $^{3,11,14,15}$. A recent review showed that increased crystallinity is good for improving mechanical, thermal, and degradation properties ${ }^{16}$. This is because crystalline polymer domains form a more ordered structure than amorphous domains, allowing additional hydrogen bonding which strengthen the material. The degree of crystallinity can be enhanced in several ways, such as annealing (e.g. ${ }^{17}$ ), or with molecular manipulation of the structure such as by fiber spinning and drawing ${ }^{18}$, or with oriented injection molding ${ }^{19}$. It has been shown that PLLA exists in a variety of crystalline structures which can appear based on processing conditions ${ }^{17}$. Even more interesting than the number of crystal phases that appear in PLLA is the enantiomeric quality of lactic acid itself. It is a chiral carboxylic acid in monomer form, and as such can take the L or D form. The units can also dimerize into L,L, D,D or the meso form. Figure 1.3 shows the chirality of the lactic acid monomer and a lactide unit ${ }^{16}$ :

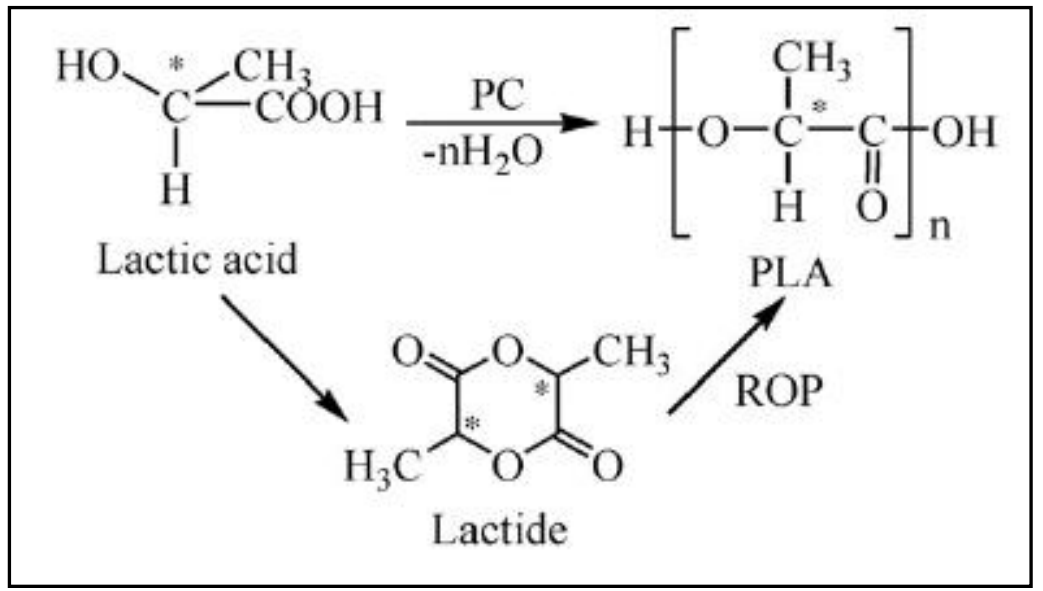

Figure 1.3: Chirality of lactic acid and lactide give rise to chirality of PLA polymer ${ }^{16}$.

In fact, Hirata et al. performed mechanical property analyses on blended PLLA/PDLA materials and found a variety of crystalline structures across compositions that could significantly improve melting temperature and DMA thermo-mechanical properties ${ }^{20}$. Unfortunately, Kolstad found during his crystallization study of poly(L-lactide-co-meso-lactide) that the presence of a mere few percent of the meso component can badly retard crystalline kinetics and reduce the overall crystallization potential $^{21}$. 
One drawback to increasing crystallinity is that ductility is typically reduced, as the crystal lattice is stiff when compared to the amorphous phase ${ }^{17}$. A simple way to increase ductility of a polymer is to introduce plasticizer into the matrix, which increases chain mobility and free volume, thus allowing the plastic additional flexibility. Ning et al. blended PLA with poly(butylene adipate) and an ester control compound and found a remarkable increase in tensile elongation at break, as well as a reduction of the glass transition temperature ${ }^{22}$. The groups of Ali et al. ${ }^{23}$ and $\mathrm{Xu}$ et al. ${ }^{24}$ both added epoxidized soybean oil (ESO) as plasticizer and discovered that viscous properties as well are reduced by this addition, so the material became more pliable as the concentration of plasticizer increased to around $30 \%$ in both studies. In a similar vein, Okamoto et al. used various low molecular weight polyester-diols to achieve the same purpose; at $20 \%$ concentration of the appropriate plasticizer, the engineering strain at break in tension was improved from $0.01 \%$ to over $7 \%$, but the glass transition temperature was reduced or in some cases appeared to be eliminated ${ }^{25}$. However, the very significant drawback of plasticizing the PLA matrix is the severe reduction of all other mechanical properties, which is antethetical to the concept of deploying PLA-based structural plastics. Another, albeit less common, technique of enhancement of PLA properties is chain extension, where additional molecular weight is gained via further polymerization occurring in either a solid or liquid phase reaction. Such a method can occur with a mixed sample ${ }^{26}$ or with the PLA itself ${ }^{27}$. Gu et al. ${ }^{27}$ synthesized their own polylactic acid and extended the chain using hexamethylene diisocyanate (HDI) as one might do if making polyurethanes, and found that the molecular weight increased alongside the mechanical and rheological properties. However, such methods are not commonly encountered, whereas the co-blending of various polymers as well as with fillers and additives is ubiquitous in the polymer industry.

This has been a burgeoning and broad field of recent work by many research teams. PLA has been previously blended with acrylonitrile-butadiene-styrene $(\mathrm{ABS})^{28}$, poly(butylene adipate-coterephthalate) $(\mathrm{PBAT})^{29,30}$, poly(butylene succinate) $(\mathrm{PBS})^{31}$, poly( $\left(\varepsilon\right.$-caprolactone) (PCL) ${ }^{32,33}$, poly(ethylene terephthalate) $(\mathrm{PET})^{34}$, and poly(glycolic acid) (PGA) $35,36,37,38,39$ among others in a variety of ways in attempts to enhance properties such that PLA may be more fully integrated into the marketplace as a commodity plastic, both for medical and structural uses. 
One such conventional polymer that may prove a good candidate for blending with PLA is polycarbonate, or PC. Polycarbonate of bisphenol A has properties that one finds lacking with PLA; it has excellent thermal resistance and is ductile for thin-walled applications. Additionally, amorphous PC is widely renowned for its optical clarity and impressive mechanical properties, including an unrivaled Izod impact strength $\left(\mathrm{e} . \mathrm{g}^{40}\right)$. This physical integrity is the reason that automotive headlamps, laboratory eyewear and face-shields, bulletproof windows, as well as audiovisual media such as CDs and DVDs are common usages of polycarbonate. Furthermore, it has excellent chemical stability when compared with PLA (e.g. $\left.{ }^{41}\right)$. Thus, the PC/PLA system is perhaps capable to address the PLA drawbacks listed above; therefore we should review whatever information is available in the literature on this topic.

\subsection{Blends of Polylactic Acid and Polycarbonate.}

In the past decade alone, there has been a small but significant amount of investigation into blending together polycarbonate and polylactic acid to produce a new material with enhanced properties. Since the new millennium began, there have been a number of patent applications filed, mostly with the European Patent Office, for a variety of co-blended PC/PLA materials ${ }^{42,43,44,45,46,47}$. These patent filings cover conventional polycarbonate as well as some novel carbonate polymers, and were usually filed by large companies such as Sony, Shimadzu, and Bayer MaterialScience. Lately, patents have been awarded to Cheil Industries (an affiliate of the Samsung Group) by both the United States and European Patent Offices (US $8232343^{48}$ and EP $2133392^{49}$ ). Their US grant filing is for the compounding of traditional polycarbonate based on BPA with PLA with the addition of small amounts of PC-co-PLA compatibilizer produced in a separate reaction, optionally with the presence of impact modifier. Improvements in mechanical properties are achieved, significantly so for the heat distortion temperature as measured by ASTM D648. European Patent 2133392 covers the same blends which are instead treated with chain extender based on siloxane, along with optional rubbery modifiers and processing aids. Improvement in properties and HDT are realized in this embodiment as well, though not as significant. Both are suggested as being suitable compositions for molded durable parts.

One point that is widely accepted about blends of PC/PLA is that the two condensation polymers are thermodynamically immiscible (e.g. ${ }^{50,51,52,53,54}$ ). In fact, Lee et al. note in the review section 
of their paper that PLA does not seem to be compatible with very many polymers ${ }^{50}$. They further state this is usually a result of large interfacial tension gradient and that material properties are dependent on the quality of this interface.

Researchers have taken essentially two competing avenues to resolve this problem. In the first camp, Lee ${ }^{50}$ et al., several patent seekers ${ }^{44,45,46,47}$, and Hung ${ }^{52}$ et al. have chosen to blend the two polymers together with compatibilizing agents. They find that addition of only a few parts per hundred resin (phr) concentration of appropriate compatibilizers is enough to improve the sharp interface observed in SEM images of immiscible material ${ }^{50,51,52}$. The alternate approach, somewhat more commonly practiced, is to fabricate a non-bisphenol A based carbonate and blend this with PLA $42,43,51,53,54$. As is typical, there are both advantages and disadvantages to this approach. These same citations show that if one opts to formulate a carbonate polymer, the properties of it may be tailorable as aliphatic carbonates can have a variety of properties. In order to achieve those properties, these particular compositions do not contain the bisphenol A moiety. BPA has been a controversial chemical in recent years in certain circles due to concerns of adverse health effects. For some, then, the psychology of using a "green" plastic is of paramount importance and these novel carbonates should suffice in their applications. On the other hand, PC owes its toughness and incredible impact strength to the bulky BPA component in its backbone, and thus materials without it simply cannot hope to maintain that strength. A third manner of improving miscibility is reactive compatibilization. PC and PLA both can undergo a so-called transesterification reaction ${ }^{54}$. This method allows chemical integration of one polymer into the structure of the other. The reaction, which takes place in the presence in multiple metal catalysts but favorably tin compounds, is integral to at least one patent application $^{47}$ and the work of Nabar and Kale ${ }^{55}$ who worked with PC and PET at elevated temperature. In doing so, they used rheological measurements and differential scanning calorimetry (DSC) to confirm that commercial PET contains enough residual catalyst to effectively transesterify the two materials into a random copolymer. While the production of transesterifed random co-polymer is usually unfavorable due to the inconsistency and unpredictability of the reaction, the proof of concept was demonstrated. All of the above citations show that a variety of methods exist to obtain PC/PLA blended polymers with properties determinable by processing conditions, in order to achieve desirable properties in the final products. 


\subsection{Objectives of the Current Work.}

Our foremost goal is to show how one may create durable plastic materials with good mechanical properties and adequate chemical resistance that contain a significant portion of renewable PLA polymer. We have identified several avenues to achieve this by addressing the issues listed in Section 1.2. Our strategies were therefore:

1. Alter the PLA crystallinity and/or add barrier materials in order to improve ductility and chemical resistance.

2. Blend PLA together with the high strength, more durable PC polymer phase to achieve improvements in mechanical properties and chemical resistance.

3. Fabricate a blend with relatively high PLA loading which is still completely encapsulated with PC. This should protect the PLA and have the good properties of a PC matrix.

4. By means of different compounding conditions, alter the interfacial morphology of the blends to minimize the exposure of the PLA phase to attacking species or ions.

5. Investigate the effects of diffusion kinetics and resistance by altering sample thickness between pellets and molding parts of varied thickness.

These compounds were fabricated by melt-blending in either a twin screw extruder or an internal mixer. The effects of heat treatment and the inclusion of graphene were investigated on the chemical and hydrolytic resistance of either pellets or molded parts. A complete composition range was studied to observe the effect of encapsulations. We set out to determine which treatment or combination of treatments would prove most effective in generating durable materials. 


\section{CHAPTER 2}

\section{LITERATURE REVIEW}

\subsection{Scope of the Research.}

As stated in Chapter 1, the ultimate goal of this work was to determine a method to produce durable plastic goods containing significant renewable or green content. To do so, we must answer a common question asked by materials scientists: Can we understand the processingstructure-property relationships of the materials? Therefore, a literature search that covers the listed drawbacks of PLA polymer and previous attempts to improve the material properties is necessary.

The major issues listed in Chapter 1 about PLA shortcomings can be considered binary; poor mechanical properties and poor chemical and hydrolytic stability. Since it is much easier to address lacking mechanical properties, the first item to investigate will be the stability of PLA.

\subsection{Degradation of PLA and PLA-Based Materials.}

PLA has been developed as a biodegradable, renewable material to be environmentally friendly. However, this poses a problem in creating PLA-based durable goods. Being a polyester material, it will degrade via the uptake of $\mathrm{H}_{2} \mathrm{O}$ which proceeds to hydrolyze ester bonds via the

reverse Fischer esterification reaction ${ }^{56}$. This may take place in the presence of acidic, neutral or basic media. Differing mechanisms have been proposed for degradation depending on solution $\mathrm{pH}$, as illustrated in Figure 2.1: 


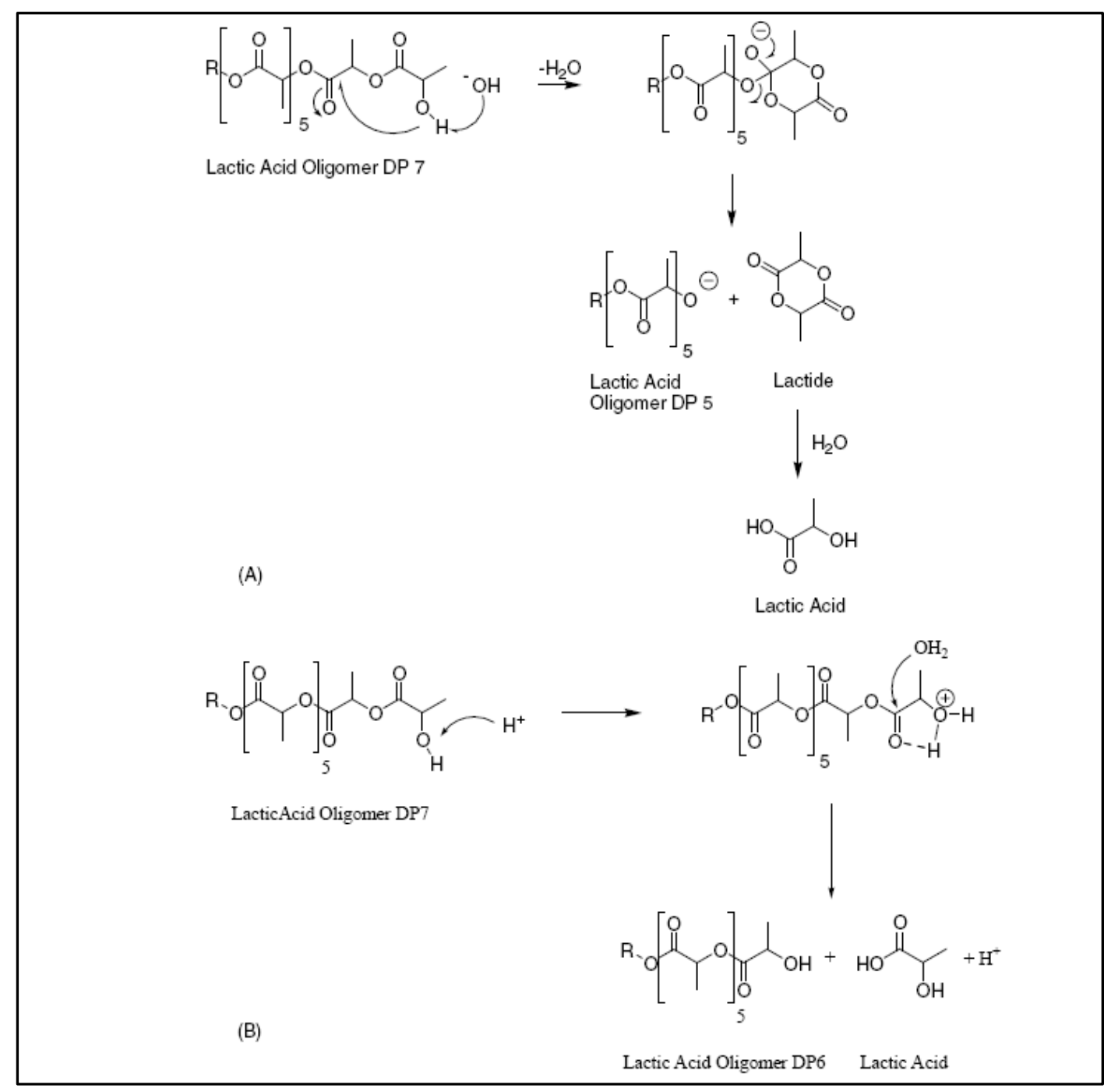

Figure 2.1: Possible mechanisms for PLA degradation ${ }^{56}$.

The same authors degraded these oligomers over a $\mathrm{pH}$ range to determine if the degradation kinetics changed, as shown in Figure 2.2: 


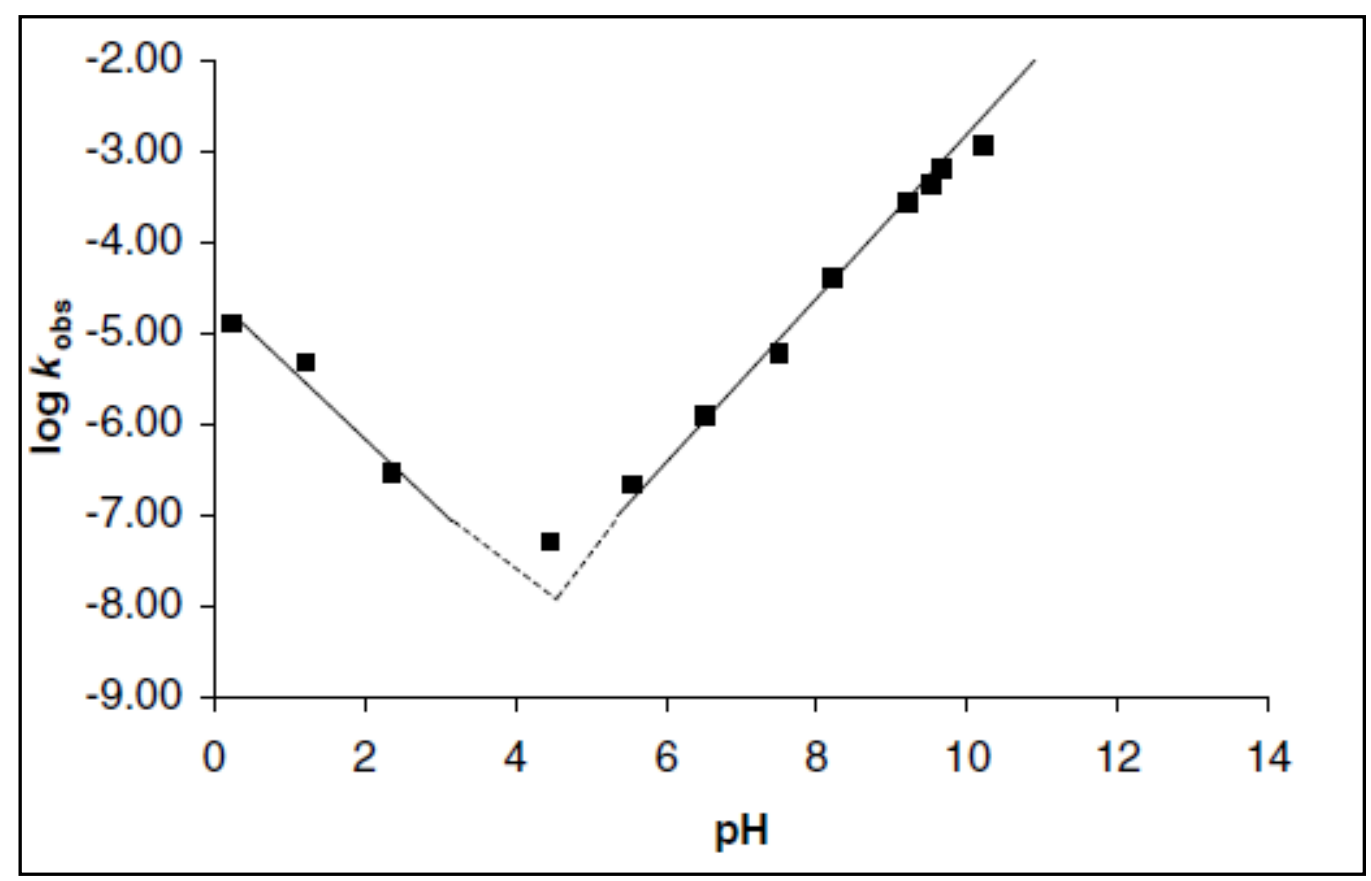

Figure 2.2: Effect of $\mathrm{pH}$ on degradation rate of $\mathrm{PLA}^{56}$.

This study was performed at $37^{\circ} \mathrm{C}$. The diverging lines indicate that a different mechanism begins taking place around $\mathrm{pH} 4$, as the rate passes through a minimum. They posit that in the range above this point, the degradation is catalyzed by hydroxyl ion, and is proton catalyzed below, as shown in Figure 2.1. Because the slopes of the lines are roughly unity, the reaction is first order with respect to the concentration of the attacking hydrogen or hydroxyl ions.

Another set of studies used very small microspheres of PLA for drug delivery purposes ${ }^{57,58}$. Since these particles were roughly $1.5 \mu \mathrm{m}$ in diameter, diffusional resistances would be negligible and would give a true picture of the stability of the spheres. They found that as the $\mathrm{pH}$ moves further from neutral (highly acidic or basic), the rate of molecular weight loss increased, as shown in Figure 2.3: 


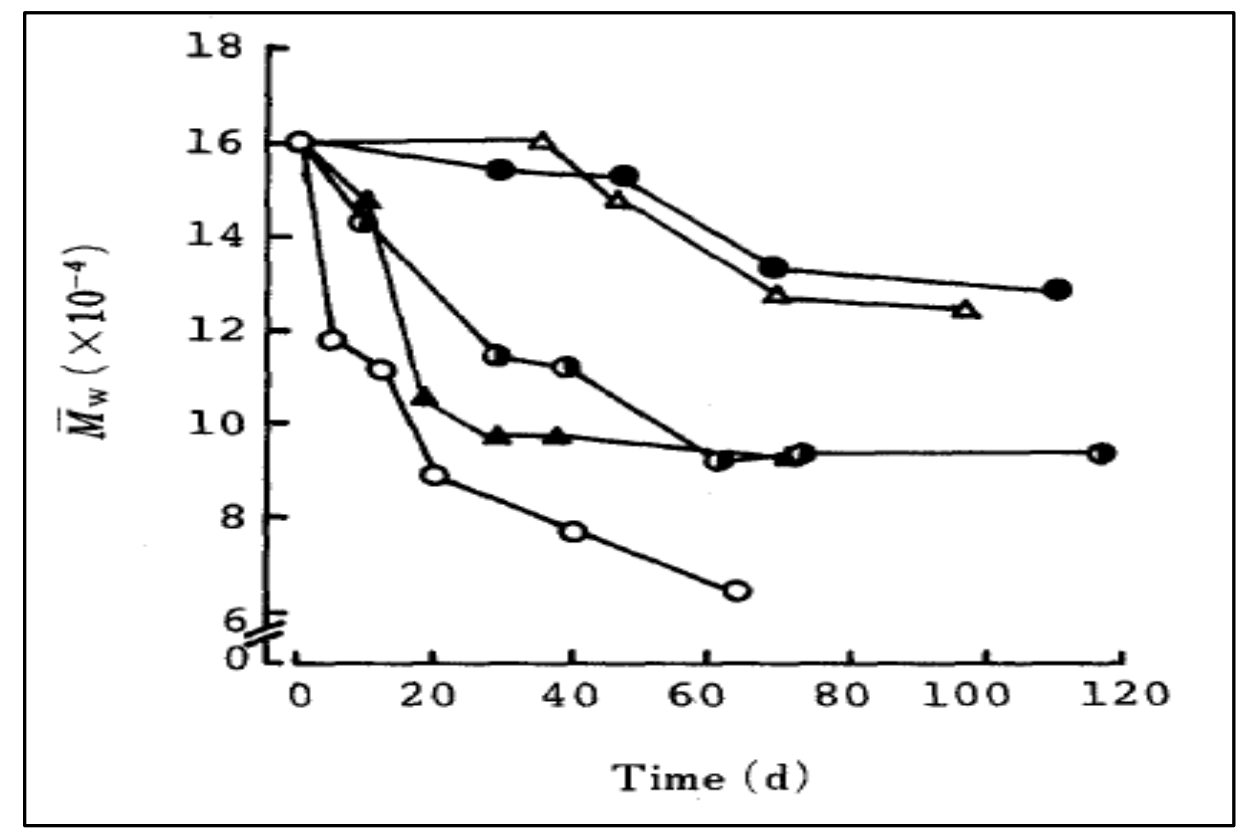

Figure $2.3 \mathrm{pH}$ dependence of PLA microsphere degradation ${ }^{57}$.

$\mathrm{pH}$ values in this study ranged from 1.6 (black triangle) to 9.6 (open circle). The specimens with $\mathrm{pH}$ around 3 (open triangle) and 5 (black circle) appeared to be the most stable, in agreement with the study above ${ }^{56}$. The half-filled circles in the above figure are at $\mathrm{pH}$ 7.4. Makino et al. also tested the solution media for lactic acid residue concentration, and found that essentially none is present except for the case of degradation near $\mathrm{pH} 10$. This was deemed to have been caused by the base catalyzed reaction "unzipping" the PLA backbone, and by the lactic acid units diffusing out of the bulk and into the liquid surroundings. This data set also confirmed the findings of de Jong et al. above, where the degradation rate of the acid-based polymer was fastest in highly basic media. Additional relevant findings from their 1985 paper were an Arrhenius style increase in degradation rate with temperature, and that poly(D,L-lactide) is slightly more susceptible to degradation than poly(L-lactide), presumably due to increased atacticity of the chain and hence lower crystallinity ${ }^{57}$. In the subsequent article, they determined that acid and base preferentially degrade different segments of the polymer; acidic media go after smaller molecular weight fragments in the bulk, while alkaline solutions are able to attack all segments of the backbone ${ }^{58}$.

These articles dealt with slow degradation of PLA material for drug delivery. For very aggressive hydrolysis conditions, however, Mohd-Adnan et al. carried out PLA hydrolysis in 
high pressure steam at up to $130^{\circ} \mathrm{C}$ and showed the material could be recycled back into lactic acid oligomers in less than 24 hours $^{59}$. Figure 2.4 describes the molecular weight loss:

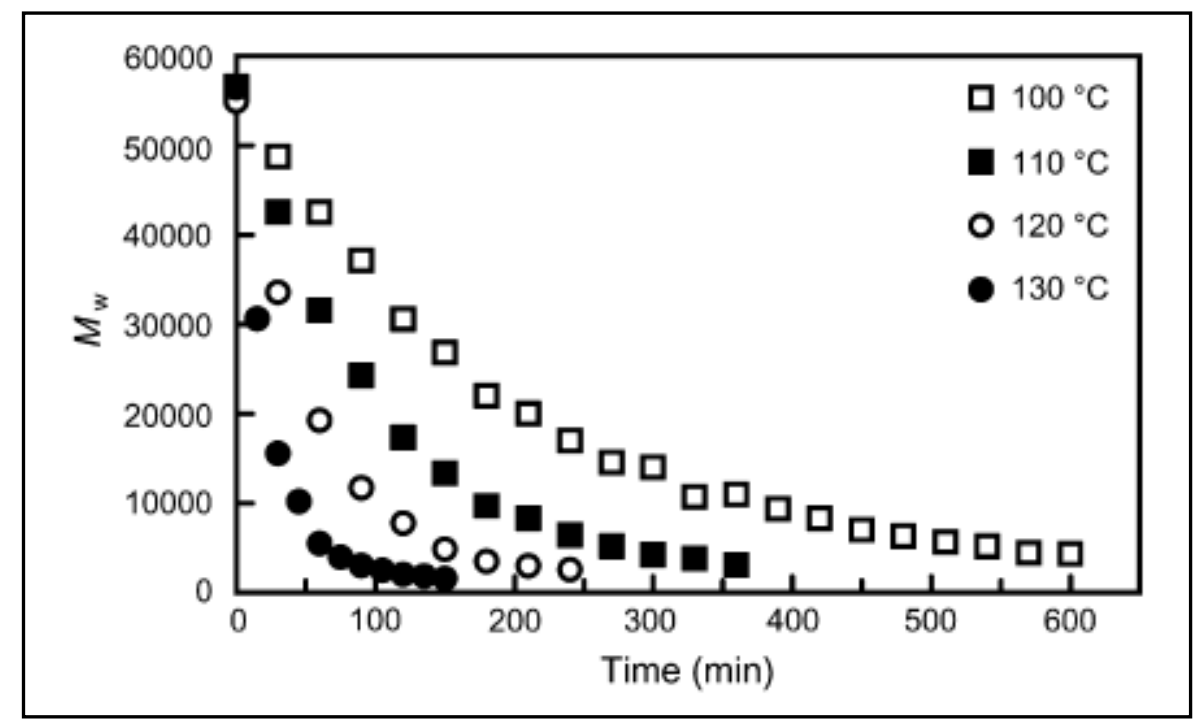

Figure 2.4: High pressure steam degradation of PLA is quite rapid ${ }^{59}$.

Thus it is clear that PLA without treatment or blending is an unsuitable material for use in durable applications, and so we must investigate the literature for blended or treated materials.

As was mentioned previously, polylactic acid has commonly been blended with PGA for biomedical applications, and several studies are available on the degradation properties of these blends. While most were blended to have relatively poor durability so they could degrade more quickly, it is still worthwhile to examine the results. Cohen et al. prepared 75/25 PLA/PGA drug delivery microspheres and degraded them under in vitro conditions, finding only small fragments remaining after about 76 days $^{35}$. The small spheres first underwent surface degradation until the interior was exposed and could diffuse away. Dunne et al. used a PLGA copolymer at similar conditions and found the materials had degraded to less than $20 \%$ of the original mass after about 100 days at $37^{\circ} \mathrm{C}$ and $\mathrm{pH} 7.4^{36}$. Fu et al. drew PLGA fibers to various crystallinities and degraded them at $40.9^{\circ} \mathrm{C}$ and $\mathrm{pH} 7.79$ where they lost strength very rapidly, shown here in Figure $2.5^{37}$ : 


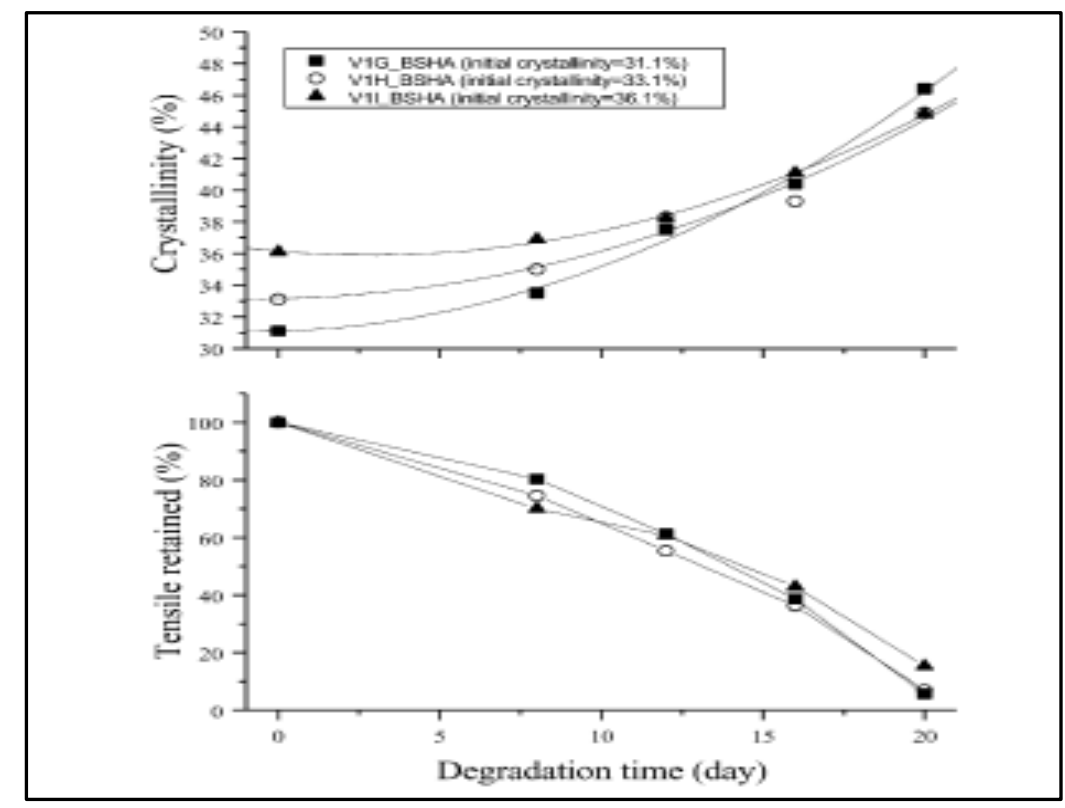

Figure 2.5: Crystallinity and tensile strength of degraded PGLA fibers ${ }^{37}$.

The effect of initial crystallinity did not seem significant in this study. Li used blends of PGA with PLA of various (D,L) compositions for in vitro degradation testing ${ }^{38}$. The parallelepipedshaped samples degraded in a hollowing out fashion if they were amorphous, indicating that the interiors were eroded first in a bulk manner. X-ray diffraction (XRD) was used to confirm that samples became more crystalline as degradation proceeded, showing again that crystalline specimens resist attack. Further, a bi-modal distribution of molecular weights was discovered with GPC, because chains of initially very high molecular weight are also quite resistant to attack, due to the lower concentration of vulnerable end groups. The addition of PGA to PLA reduced the resistance to degradation in some cases quite severely; 100\% PLLA had a degradation half-life of 110 weeks in this phosphate buffer, while that of 85/15 PLLA/PGA was only twenty weeks ${ }^{38}$. A final study considering blended PGA and 50:50 poly(D,L-lactide) evaluated under what conditions this biodegradable composite would erode from the surface or the bulk ${ }^{60}$. It was determined that when the rate of diffusion into the matrix is fast with respect to the rate of ester bond scission, then attack will take place in the bulk; otherwise, if the reaction is much faster than diffusion, the surface will be the area of degradation. This is plotted in Figure 2.6: 


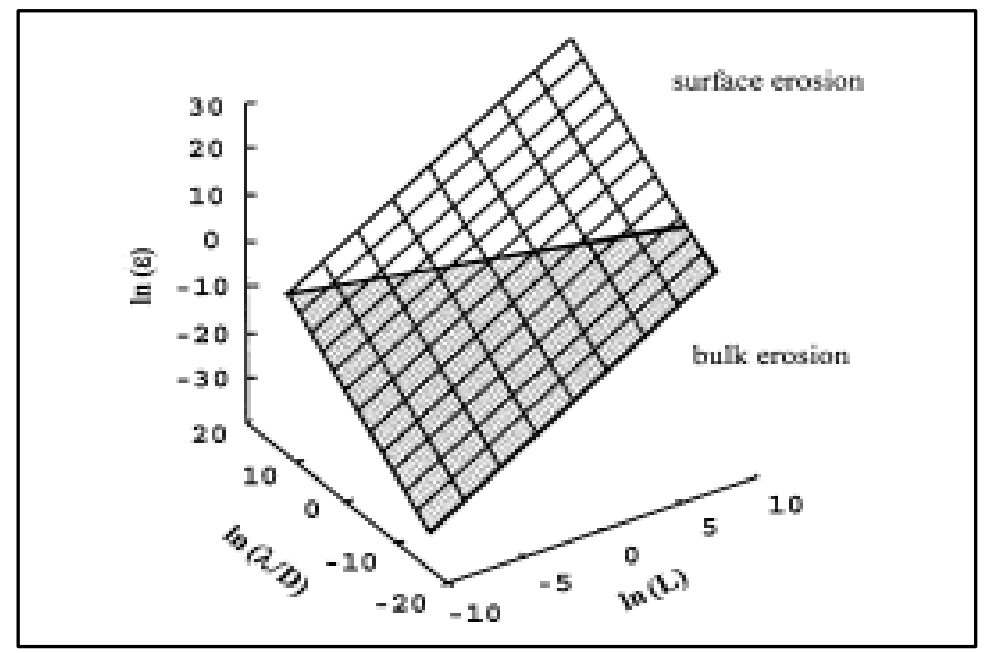

Figure 2.6: Surface or bulk erosion may take place for a given material ${ }^{60}$.

This turned out to be a highly unstable material; for the blended 50/50 PGA/PLA with molecular weight of 14000 , more than $80 \%$ of the original mass had dissolved away within 24 hours. It was also noted that the PGA/PLA blends were more susceptible to degradation than was PLA alone.

Some of these studies also considered the effects of PLA or overall composite crystallinity on the rate of degradation. Kim et al. processed PLA by fiber spinning at various speeds to provide molecular orientation which altered the crystallinity ${ }^{18}$. Increasing the so-called take-up speed could make the material much more crystalline, shown in Figure 2.7: 


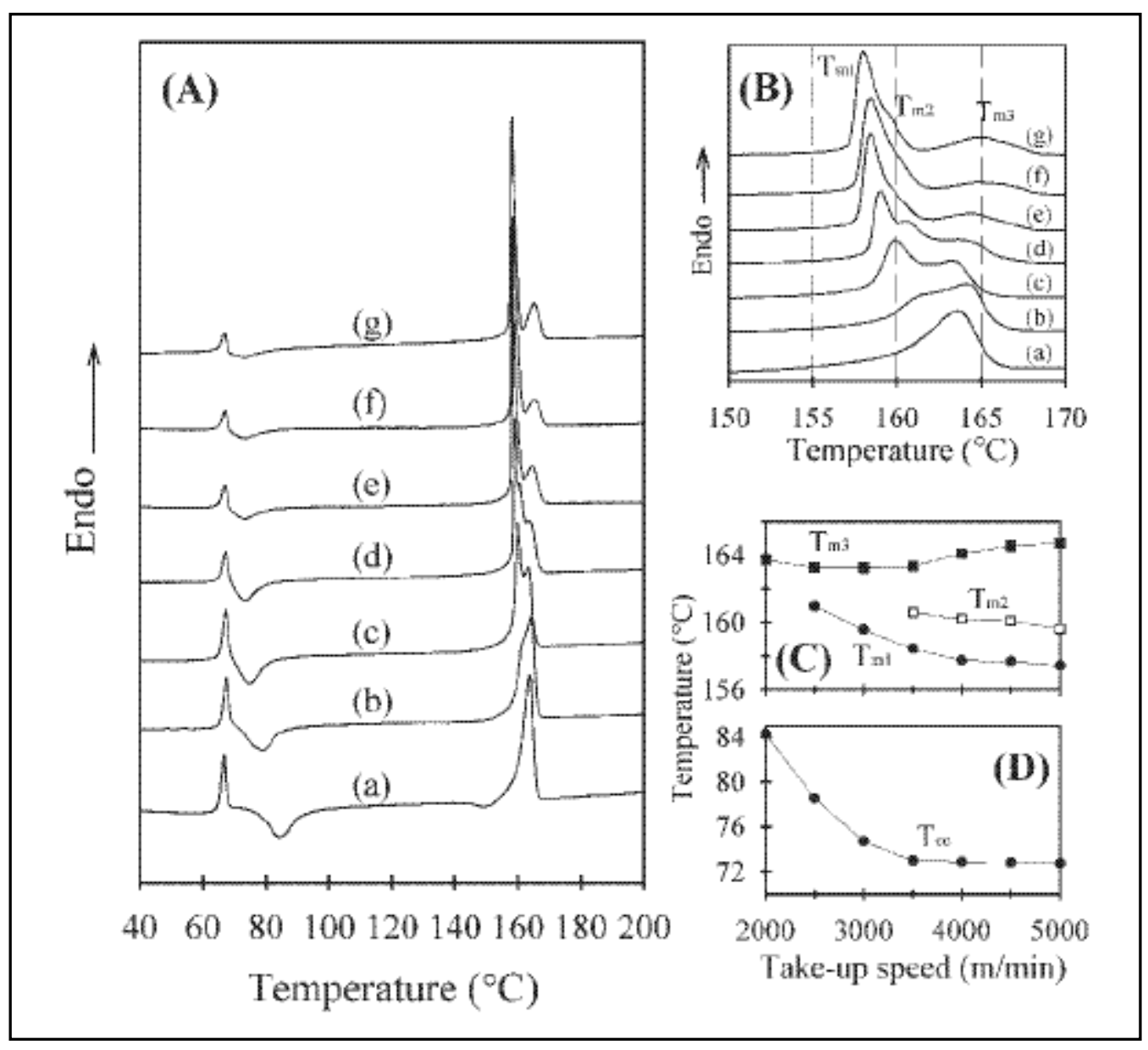

Figure 2.7: Thermal properties change with molecular orientation ${ }^{18}$.

The plots labeled $A$ and $B$ in the figure are DSC curves while $C$ and $D$ give the crystal melting and glass transition temperature. These materials were then subjected to hydrolysis in boiling water, after which the mechanical properties were measured in tension: 


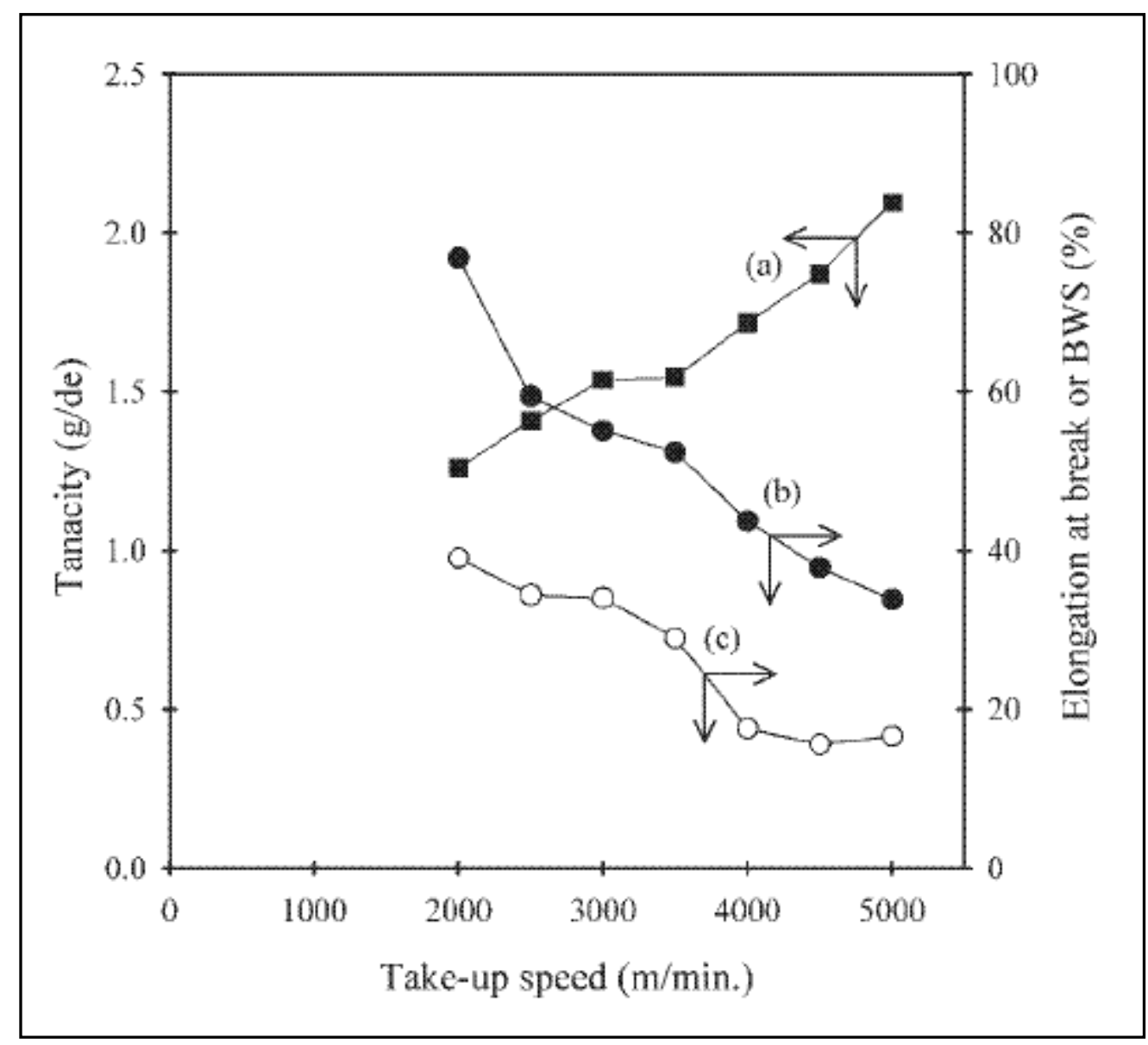

Figure 2.8: Changes in mechanical properties after boiling water exposure ${ }^{18}$.

Figure 2.8 shows that the more crystalline materials retained ductility (or elongation at break, line $b$ ) and strength after hydrolytic degradation due to molecular orientation which reduced the capacity of water to diffuse into the bulk. A study done by Zhang et al. investigated both amorphous and semi-crystalline PLA materials over a range of $\mathrm{pH}$ and time duration ${ }^{61}$. They found that the materials undergo two-stage degradation with both surface and bulk erosion steps, with either mass or molecular weight loss as shown in Figure 2.9: 


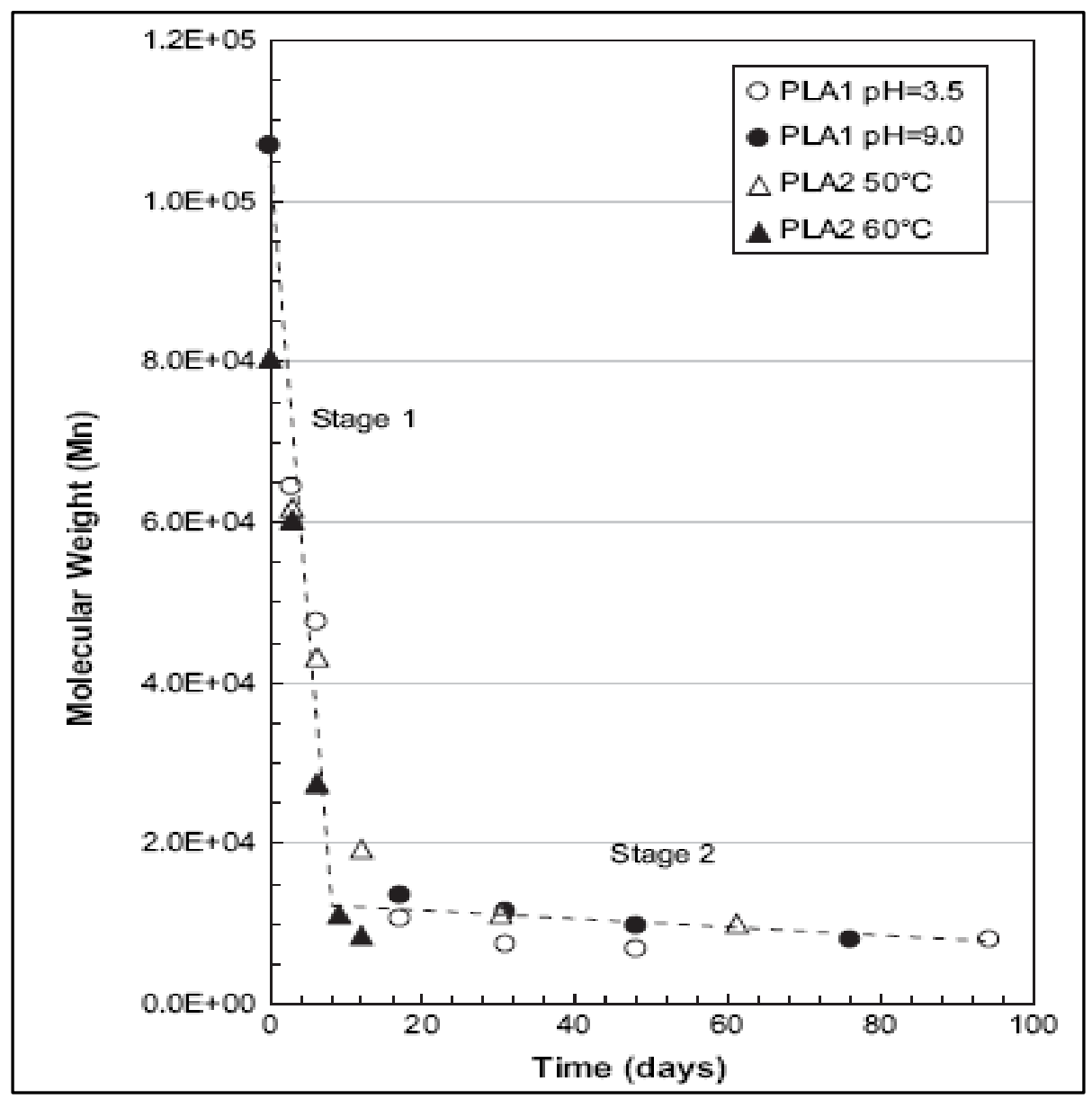

Figure 2.9: PLA degradation in acid, neutral and basic media ${ }^{61}$.

Stage 1 is where bulk diffusion occurred and molecular weight was lost, followed by the material actually falling apart with mass loss taking place. The effect of increased PLA crystallinity was not great in this study. Zhou and Xanthos added nano-clays to amorphous, semi-crystalline and blended PLA samples and degraded the materials in base above and below the glass transition temperature ${ }^{62}$. The addition of the clay into the PLA matrix adds a diffusion resistance which increases the time required for attacking species or ions to fully permeate into the material, enhancing the stability of the composites over short timescales. They found reaction rates to increase with temperature and that while increasing crystallinity improved the resistance, the clays could act as nucleation sites for degradation and enhance the reaction rate. Harris et al. performed hydrolysis experiments with highly crystalline ( $45 \%)$ injection molded PLA specimens and found that after 8 weeks exposure to $50^{\circ} \mathrm{C}$ and $90 \%$ relative humidity, nearly all flexural integrity was gone ${ }^{63}$. As they were Ford employees, they concluded that PLA even 
when highly crystalline could not be used for durable applications like automotive parts on its own. Tsuji and coworkers wrote several articles on degradation of amorphous and crystalline PLA samples at even higher temperatures ${ }^{64,65,66}$. They showed that PLLA could be easily recycled by hydrolysis in the range of $120-190^{\circ} \mathrm{C}$, and at $190^{\circ} \mathrm{C}$ this could occur in as little as 30 minutes ${ }^{64}$. As will be addressed in the next section, polycarbonate must be exposed to much more aggressive temperatures and humidity for significant hydrolysis to occur. Okamoto et al. at Toyota added nano-clays and crystal growth accelerating agents to find improvements in mechanical properties, though they did not study degradation of the samples ${ }^{67}$. Lastly, while not strictly a test of hydrolytic stability, Rhim et al. blended various nano-clays into PLA and showed a reduction in water vapor transmission through the film in Figure 2.1068:

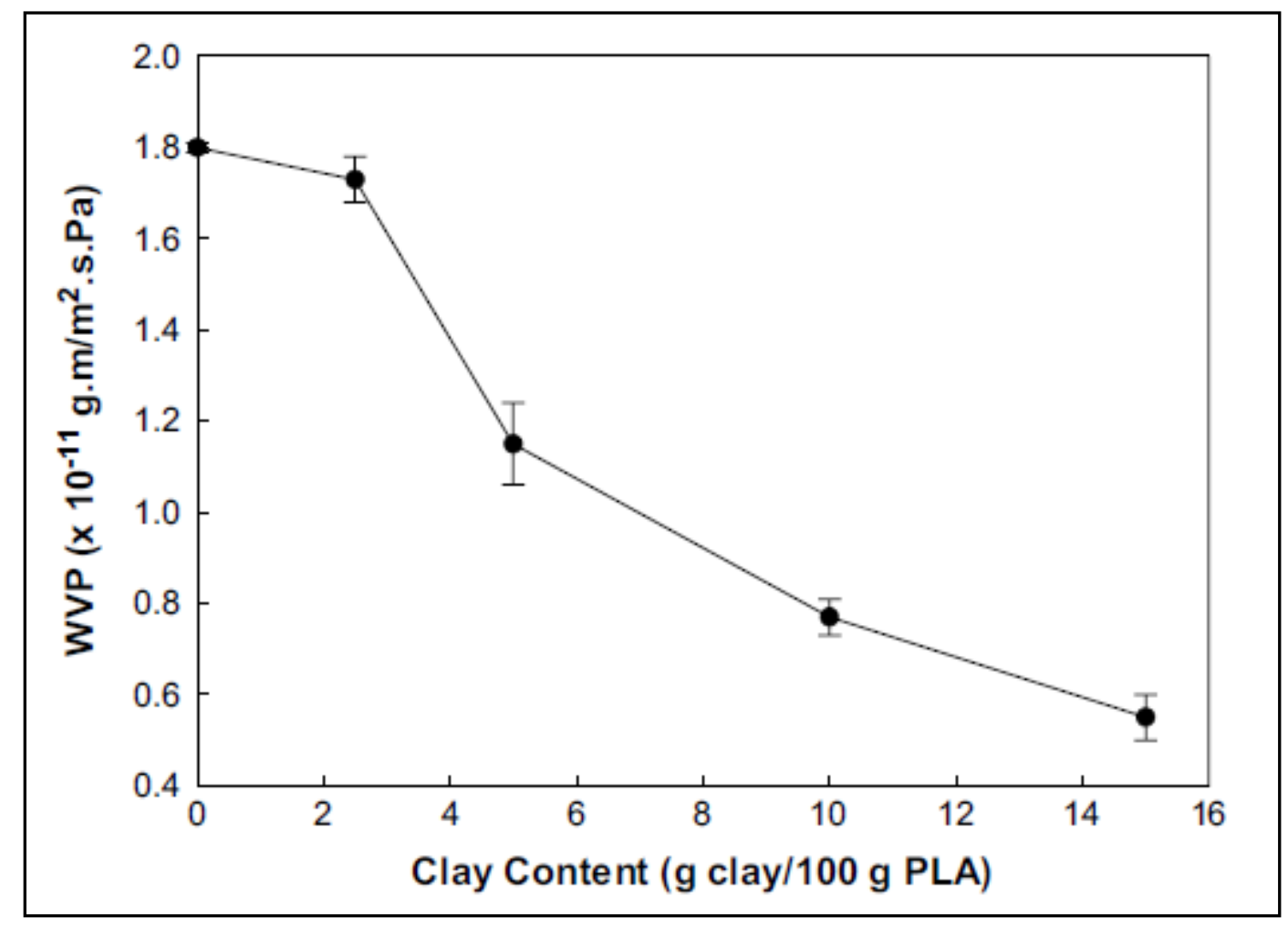

Figure 2.10: Addition of clay reduces $\mathrm{H}_{2} \mathrm{O}$ transmission of PLA films ${ }^{68}$.

Based on the findings from the literature above, it should be possible to employ singular or a combination of treatments such as compounding, annealing and adding barrier materials to enhance the hydrolysis resistance and chemical stability of PLA-based materials. Before moving to the mechanical properties of such materials, there is some literature available to confirm the assertion that blending PC with PLA would help this enhancement. 


\subsection{Degradation Behavior of Polycarbonate.}

While not as chemically unstable as polylactic acid, like any other condensation polymer PC can undergo hydrolytic degradation under appropriate conditions. In a study looking for an environmentally friendly way to recycle PC, Watanabe et al. used bomb reactors to decompose the material in the presence of oxygen-free water and steam at $300^{\circ} \mathrm{C}^{41}$. Under this extremely aggressive, high pressure environment, PC could be decomposed significantly in as little as 5 minutes as depicted in Figure 2.11:

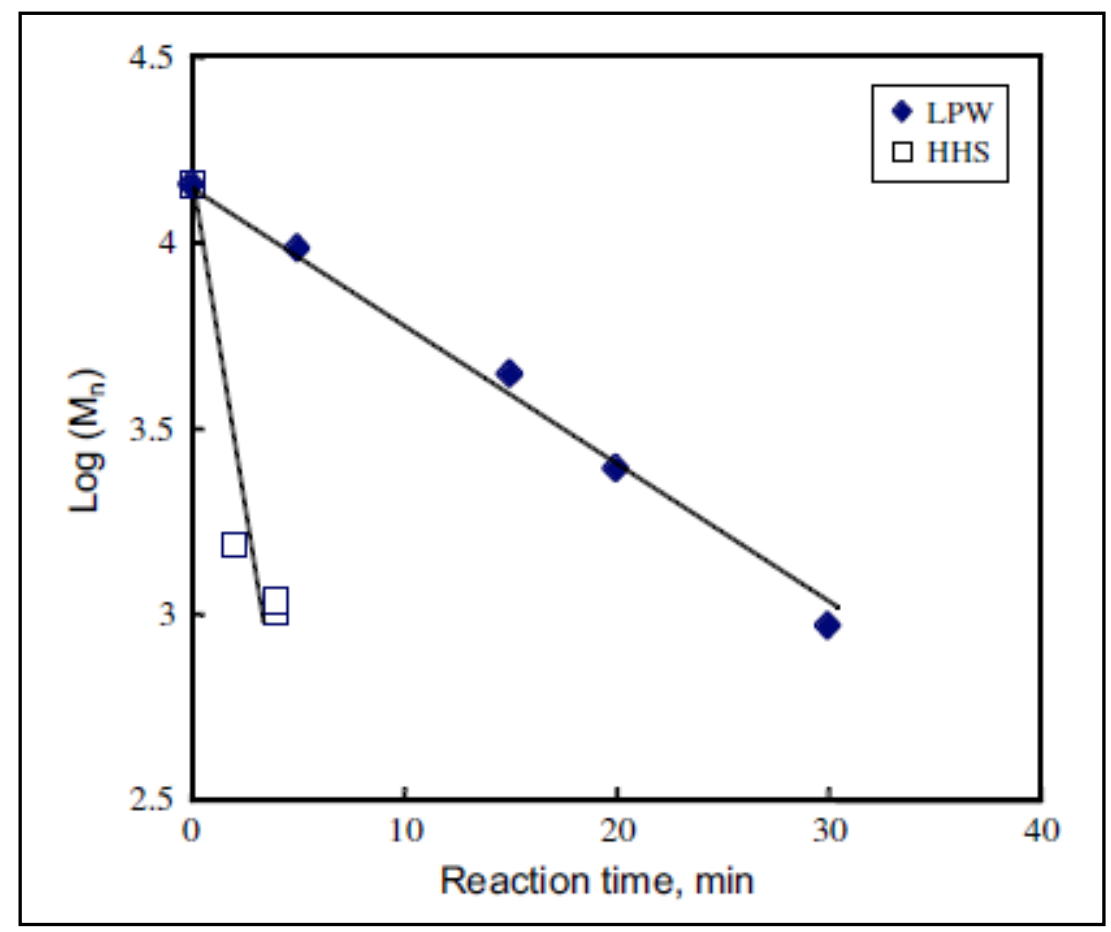

Figure 2.11: Hydrolysis of $\mathrm{PC}$ in a $300^{\circ} \mathrm{C}$ water/steam bomb reactor ${ }^{41}$.

They showed that the molecular weight loss was much more rapid in the steam phase, and suggested that the diffusion rate of the attacking steam was higher due to lower viscosity. Tagaya et al. performed a similar study using subcritical and supercritical water up to $430^{\circ} \mathrm{C}$ and found similar results ${ }^{69}$. Zinbo et al. used commercially available Calibre ${ }^{\circledR}$ and Lexan ${ }^{\circledR}$ polycarbonate samples and performed hydrolytic aging between $65-93^{\circ} \mathrm{C}$ at several relative humidities between 56-100\% $\%^{70}$. They determined that PC samples lose molecular weight by a first order reaction in this range that is of the common Arrhenius form:

$$
\frac{\left(\overline{M_{w}}\right)_{t}}{\left(\overline{M_{w}}\right)_{0}}=e^{-k t}
$$


Where $M_{w}$ is the weight-average molecular weight, $k$ is a kinetic parameter and $t$ is the exposure time. Tabulations of the kinetics for various exposures and samples were listed:

Table 2.1: Hydrolysis kinetics of polycarbonate ${ }^{70}$.

\begin{tabular}{|cccr|}
\hline \multirow{2}{*}{$\begin{array}{c}\text { Relative } \\
\text { Humidity (RH in \%) }\end{array}$} & \multicolumn{3}{c|}{$\mathbf{k \times 1 0 ^ { 3 } \text { (day } ^ { - 1 } \text { ) }}$} \\
\cline { 2 - 4 } & At $\mathbf{5 0 ^ { \circ } \mathbf { C }}$ & At $\mathbf{8 0} 0^{\circ} \mathbf{C}$ & At $\mathbf{1 0 0}{ }^{\circ} \mathbf{C}$ \\
\hline 56 & 0.0419 & 0.4875 & 2.0100 \\
73 & 0.0534 & 0.6209 & 2.5600 \\
87 & 0.0761 & 0.8853 & 3.6500 \\
95 & 0.1270 & 1.4771 & 6.0900 \\
100 & 0.2584 & 3.0053 & 12.3905 \\
\hline
\end{tabular}

While the effect of temperature is obviously important, the rate still increases in each column by about a factor of about five from the lowest to highest humidity. They estimate that the half-life (based on $M_{w}$ ) at $50^{\circ} \mathrm{C}$ and $90 \% \mathrm{RH}$ (the same conditions as Harris et al. ${ }^{63}$ ) to be 8.4 years for Lexan 141 and 19.9 years for Calibre 300-15. It has been suggested that 10 years outdoor exposure is the minimum acceptable service lifetime for automotive grade plastics ${ }^{63}$; therefore both PC grades exceed the standard and are much more stable than PLA. Therefore it seems that PC would be an excellent choice for a highly durable polymer phase to blend with PLA to enhance the hydrolytic stability of the composites.

\subsection{Mechanical Properties of the Materials.}

\subsubsection{Mechanical Properties of PLA-Based Blended Materials.}

While it has been successfully applied for biodegradable and non-extreme condition durable goods, polylactic acid must be enhanced or modified in terms of its material properties for use as a structural component, and is often blended with other plastics and additives to do so. Most of these are common engineering or industrial polymers, but some exotic materials can be found as well. A number of rigorous review articles can be found to outline some of the information that may be addressed herein; Yu et al. ${ }^{2}$ and Rasal et al. ${ }^{7}$ are good examples listing the varieties of PLA based plastics available. However, many of those described therein are for high renewable and biodegradable content applications, not durable ones. 
PLA has long been mixed with other bio-sourced materials since it became popular for use in medicine. One such example is PGA as was listed earlier, for degradation properties $35,36,37,38,39$, and is therefore certainly not durable, but physical integrity of the sutures or drug delivery capsules is paramount to achieving the correct dissolution profile. Fu et al. ${ }^{37}$ drew fibers of PGA-co-lactide and tested them for thermo-physical properties as in vitro degradation took place. They found that materials having the greatest initial crystallinity had the best strength over the course of degradation, and crystallinity tends to increase while strength is lost as degradation proceeds. This shows that amorphous segments are preferentially eroded away. PCL has also been co-blended with PLA frequently. Takayama showed that the two immiscible materials can be compatibilized together, and further that via annealing the flexural modulus can be improved; this of course reduces the flexural strength by way of inducing brittleness. Fracture energy was greatly improved with the addition of their triisocyanate compatibilizing catalyst ${ }^{33}$. Viljanmaa et al. ${ }^{71}$ studied PCL/PLA blends as hot melt adhesives and found that while the properties are adequate, the samples degrade rapidly and are not shelf stable. By chemically protecting the chain ends with peroxide modification and acetyl end-capping, this instability could be somewhat mitigated. Broz et al. ${ }^{72}$ experimented at NIST with a full range of compositions of PCL/PLA, and found them to form a lower critical solution temperature system with limited miscibility. Neither DSC nor SEM could confirm any substantial miscibility. The PBS/PLA blend was investigated by Yokohara and though the polymers were totally immiscible, PBS was capable of nucleating the crystallization of PLA ${ }^{31}$.

More durable conventional PLA blends have also been investigated. Li added acrylonitrilebutadiene-styrene (ABS) with PLLA to improve toughness, but they actually discovered the system to be antagonistic without compatibilization. Once compatibilized with styreneacryonitrile (SAN)-grafted-maleic anhydride in the presence of a bromine catalyst, the system showed an improvement in mechanical and thermal properties, as shown in Figure $2.12^{28}$ : 


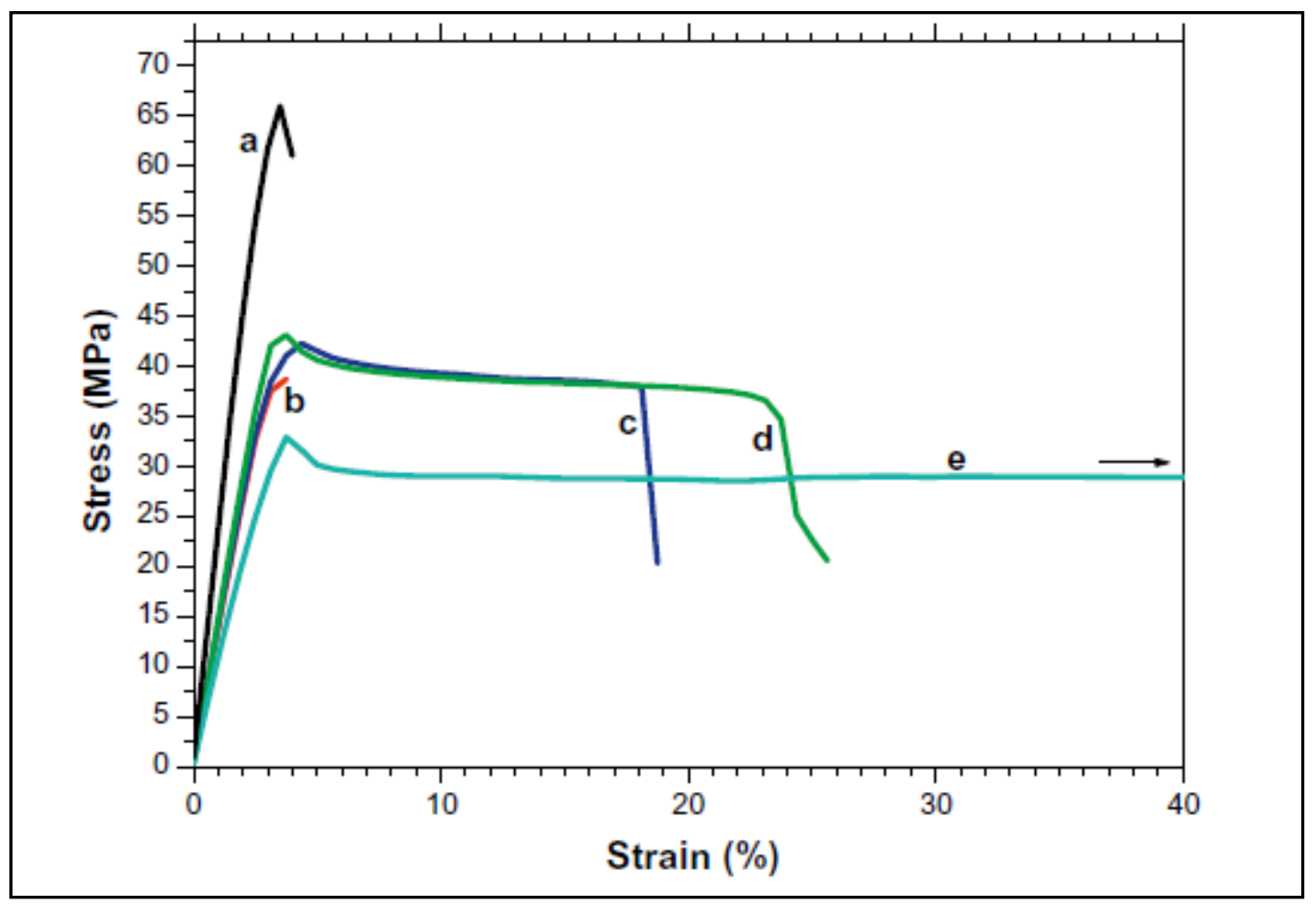

Figure 2.12: Compatibilization of PLLA/ABS blends enhances properties ${ }^{28}$.

Curve $A$ is PLLA, $B$ is 50/50 PLLA/ABS, $C$ and $D$ are compatibilized blends and $E$ is neat ABS. They included TEM images which confirmed that the compatibilization improved the morphology by allowing phase mixing. Leclair investigated the HDPE/PC/PS system and found that very poor interfacial contact and phase inversion lead to these antagonistic behaviors ${ }^{73}$. Zhang found PLA and poly(methyl methacrylate) (PMMA) to exhibit similarly poor miscibility, when the PLA component was crystalline. Apparently the crystals were capable of expelling the PMMA domain from the PLA matrix. Amorphous DL-containing polylactic acid did not exhibit this phenomenon ${ }^{74}$.

Some of the recent patents and patent applications listed in the introduction addressed the blending of PLA with conventional or unconventional polycarbonate materials. Lee et al. found that differing types of compatibilizers gave improvements in the impact, flexural, and tensile strength of 70/30 PC/PLA, but at differing optima in the range of single parts per hundred resin (phr) concentrations ${ }^{45}$. These agents also modestly helped the blends resist hydrolytic degradation. Ikehara et al. used a biodegradable semi-crystalline polycarbonate they called PEC (polyester carbonate) to verify that spherulites of the two polymers can interpenetrate, which 
could improve compatibility ${ }^{51}$. Hung et al. blended PC and PLA with an epoxidizing catalyst, which eliminated the double glass transition hallmark of immiscibility and improved the storage modulus $^{52}$. Other researchers compounded varieties of PLA with aliphatic carbonate polymers, though with an unexpected research focus. Zhou added a low molecular weight carbonate to plasticize (D,L)-lactide, nearly obliterating its strength and modulus while enhancing the elongation enough to make the copolymer suitable as a packaging material ${ }^{53}$. Another group used a polycarbonate-diol and chain extension scheme to achieve high ductility at the expense of strength $^{26}$.

Besides polymeric blends, high strength organic and inorganic additives have also been used to attempt to create durable PLA compositions. Cellulose ${ }^{10}$, carbon nanotubes $^{9,29}$, several types of clay $^{75,76}$, and natural plant fibers ${ }^{77,78}$ have been integrated into the PLA matrix to improve the heat deflection temperature, ductility and barrier properties. Clays and nanotubes have the added bonus of serving as nucleation agents for enhancing PLA crystallization, while the natural fibers add heat resistance and ductility. However, a major caveat with certain additives is the tendency to aggregate rather than homogeneously disperse throughout the matrix, even at loadings as low as 3-5 weight percent (e.g. ${ }^{79}$ ). This leads to zones of stress concentration, causing materials to become brittle. The work of Rhim et al. presented earlier included mechanical property testing into the water vapor permeability study, which illustrated the embrittlement effect: 


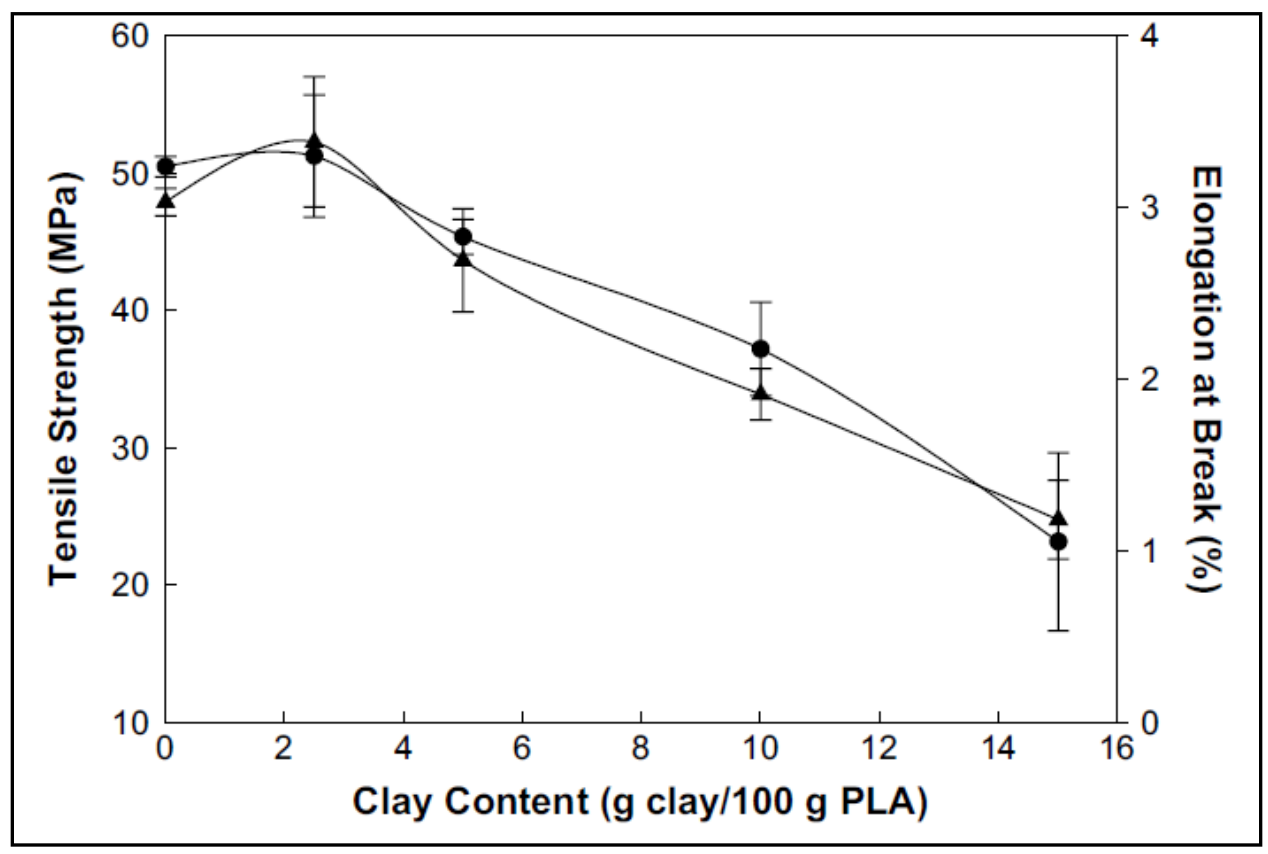

Figure 2.13: Inappropriate loading of clay causes properties to decrease ${ }^{68}$.

In Figure 2.13 above, the tensile strength is represented by the circles and elongation at break by the triangles. Narimissa et al. added up to $10 \%$ graphene platelets to PLA in a twin screw extruder and also noticed a decrease in mechanical properties such as tensile strength and modulus ${ }^{79}$ :

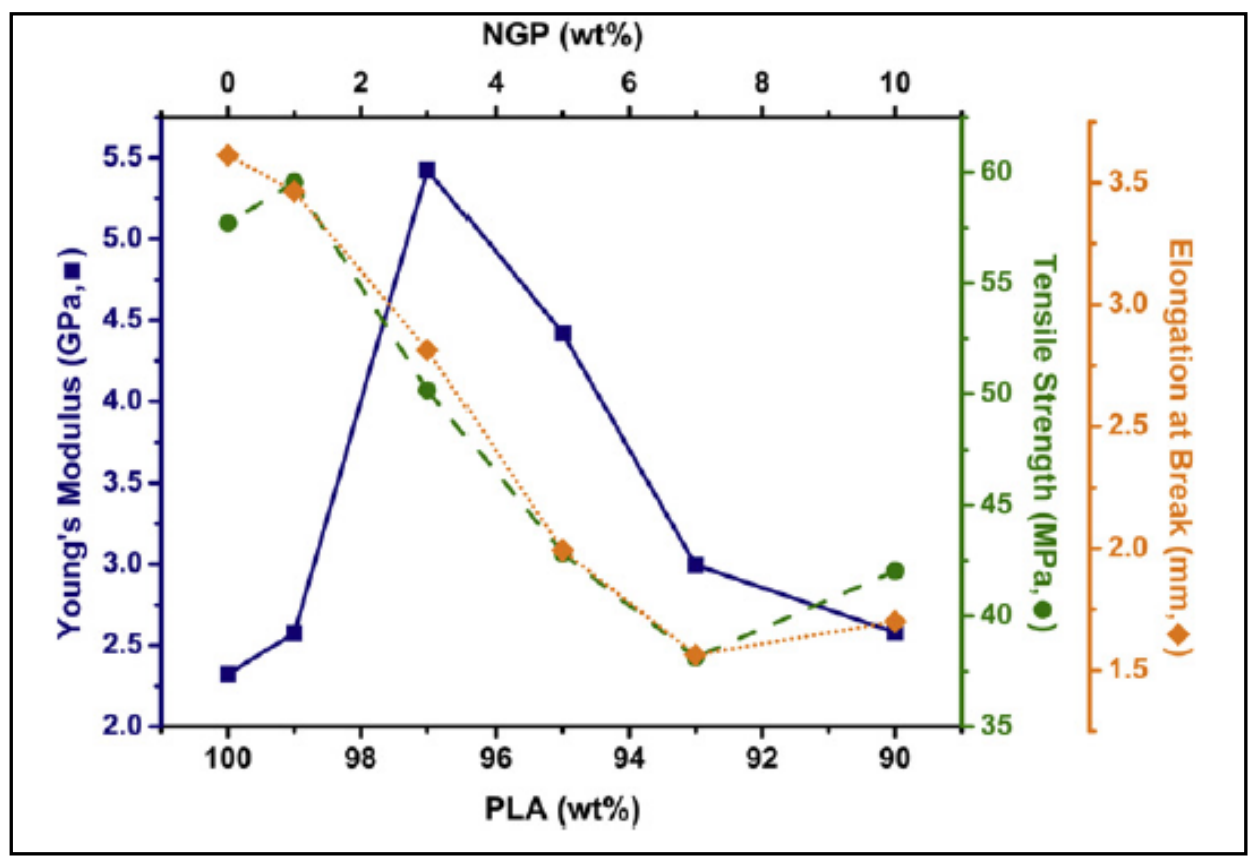

Figure 2.14: Loss of properties due to aggregation of nano-scale fillers ${ }^{79}$. 
It appears that about 2-3\% loading of nano-fillers produces the best effects, which is clear from the maximum modulus in Figure 2.14. With these publications in mind, we know that by blending PLA into a composite with a more durable material or by judiciously adding high strength filler, it is possible to enhance the material properties. The next item to address is the effect of polymer crystallinity on material properties.

\subsection{Crystalline/Thermal Properties of Polymeric Materials.}

Many polymeric blends contain individual components with divergent thermocrystalline properties. PLA in most of forms is semi-crystalline while PC is amorphous. Blended materials with an amorphous/crystalline character have been studied before, and we will address literature about these systems. This section will cover the theory of crystallization and describe the crystalline properties of PLA and PLA-based blends.

\subsubsection{Theoretical Aspects of Crystallization.}

While polymer crystallization is the topic of this work, many other materials such as metals and minerals also undergo crystallization. The theory that underpins the phenomena at play is fundamentally identical, being driven by thermodynamic means. Much of this theory of crystallization is due to the work of Melvin Avrami, who lends his name to it ${ }^{80,81,82}$.

Crystallization of polymeric materials occurs in order to lower the overall energy state of the material by packaging chains into a regularly structured lattice. This rearrangement takes place whenever there is sufficient molecular mobility or free volume for chains to move into place in the lattice. The temperature range over which this happens is between the glass transition temperature and the melting point ${ }^{83}$. The process may be initiated spontaneously within a material (homogeneous) or be promoted by the presence of a second phase, impurity, or some other nucleation site (heterogeneous). The mathematics involved can be found in materials or polymer textbooks (e.g. $\left.{ }^{83}\right)$.

Avrami theory begins by assuming that in a rubbery polymer, there are $\bar{N}$ nuclei from which crystals can grow from. The number of nuclei is temperature and time dependent ${ }^{80}$. The probability that a seed nucleus will become a growth nucleus is a thermally activated process:

$$
n(T)=k * \exp \left(-Q+\frac{A(T)}{R T}\right)
$$


Here, $k$ is the pre-exponential kinetic factor, $Q$ is the activation energy, and $A$ (a function also of temperature) is work required to spawn a growth nucleus. Then, the above relation may be plotted in Figure 2.15 as the contribution of both terms:

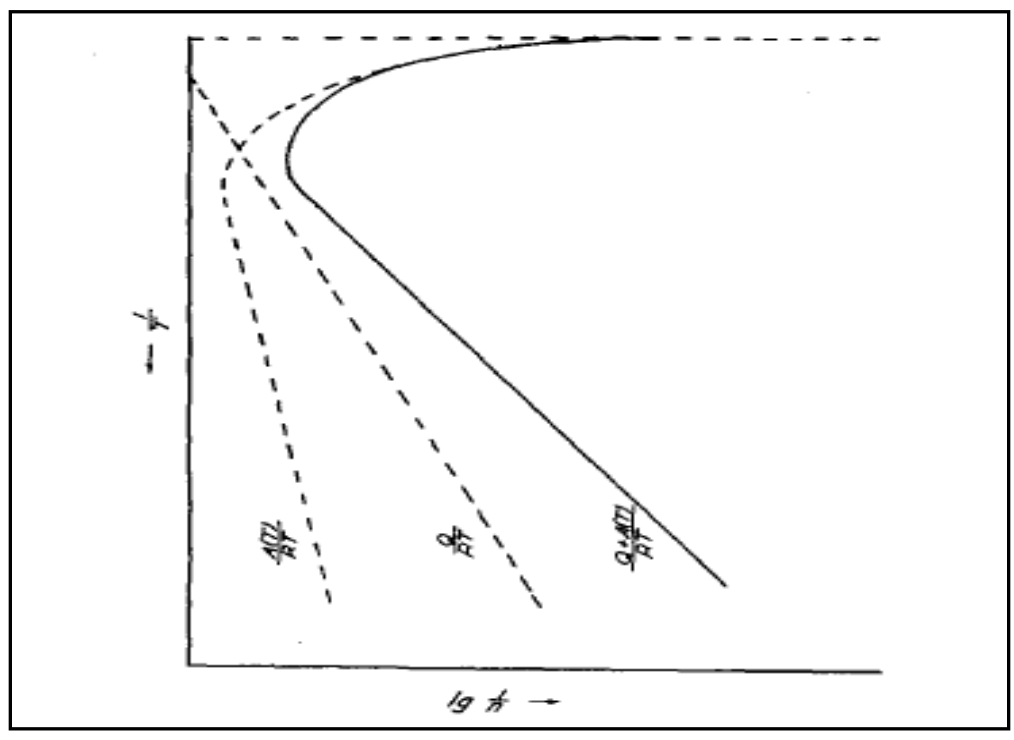

Figure 2.15: Contribution of energy terms in generation of possible crystal growth nuclei ${ }^{80}$.

Because the number of crystal nuclei may change by either exhaustion of growth or by impingement into another crystal, we may write the general differential balance on the quantity of nuclei:

$$
d N=-d N^{\prime}-d N^{\prime \prime}
$$

Where $d N^{\prime}=n N d t$ and $d N^{\prime \prime}=\bar{N} d V$. The first term is representative of time dependent germ generation and the second the enveloping of nuclei into others. During the beginning of crystallization the time term is dominant, so it can be integrated:

$$
N^{\prime}=\int_{0}^{t} n N d t=\bar{N}(1-\exp (-n t))
$$

Next, we can merge the time and $n$ parameters together so we can be write:

$$
n d t=d \tau, N(t)=N(\tau), V(t)=V(\tau)
$$

By changing from ordinary time to characteristic time, all crystallization times can be shifted onto a master curve ${ }^{80}$. This allows the rewriting of [2.3] into: 


$$
\frac{N(\tau)}{\bar{N} \exp (-\tau)}=1-V(\tau)
$$

Where $\mathrm{V}$ is the volume fraction of material that has crystallized by time $\tau$. At this point in the derivation, a new spatial coordinate $\alpha$ is introduced, which is related to the growth velocity of a crystal by:

$$
\frac{G}{n}=\alpha
$$

The purpose of defining $\alpha$ in this way is that for the so-called isokinetic range where the temperature of crystallization and the composition of the substance are constant, $\alpha$ is a constant. This provides another characteristic parameter to help in the generation of master curve for crystallization.

For many crystallizable substances, as the growth velocity increases the dimensions of growth decrease $^{81}$. This introduces shape factors into the growth of crystals in the above equations. Regardless of shape or dimension, the following may be written arbitrarily for a region in which no material has yet crystallized:

$$
\frac{v^{\prime}}{v_{1 e x}}=1-V
$$

Where $v^{\prime}$ is the volume of that arbitrary region and $v_{l e x}$ is the extended volume of a randomly chosen, overlapped crystallized region. The right hand side, assuming constant density between both solid phases, also gives the relative volume of untransformed material. This may be integrated to give:

$$
V=1-\exp \left(-V_{1 e x}\right)
$$

Under isokinetic conditions, this can be extended for three-dimensional growth ${ }^{81}$ :

$$
V_{1 e x}=\sigma \alpha^{3} \bar{N} \int_{0}^{t}(\tau-z)^{3} \exp (-z) d z
$$

Here, $\sigma$ is a shape factor, and the third order terms assume polyhedral growth and must be altered to account for growth in less than three dimensions. Equation [2.9] may be integrated to obtain the terms relating to those growth dimensions, and expressed as a Taylor series: 


$$
V_{1 e x}=\frac{6 \sigma G^{3} \bar{N}}{n^{3}}\left[\exp (-\tau)-1+\frac{\tau^{2}}{2 !}-\frac{\tau^{3}}{3 !}\right]=\frac{6 \sigma G^{3} \bar{N}}{n^{3}} E_{3}(-\tau)
$$

Where the fraction outside the bracket is abbreviated $\beta$ and the shape term $E_{i}$ is tabulated. If the assumption is made that $n$ is large (otherwise crystallization is unlikely to occur), then $\tau$ is also large and the third order term will dominate. We obtain, for three-dimensional growth:

$$
V=1-\exp \left(-\sigma G^{3} \bar{N} t^{3}\right)
$$

This can also be written in a more generic form:

$$
V(t)=1-\exp \left(-k t^{n}\right)
$$

This is the most commonly seen form of the Avrami expression, with $k$ as a kinetic parameter and $n$ is related to the crystalline shape. Avrami plotted the possible forms of this expression in Figure $2.16^{81}$ :

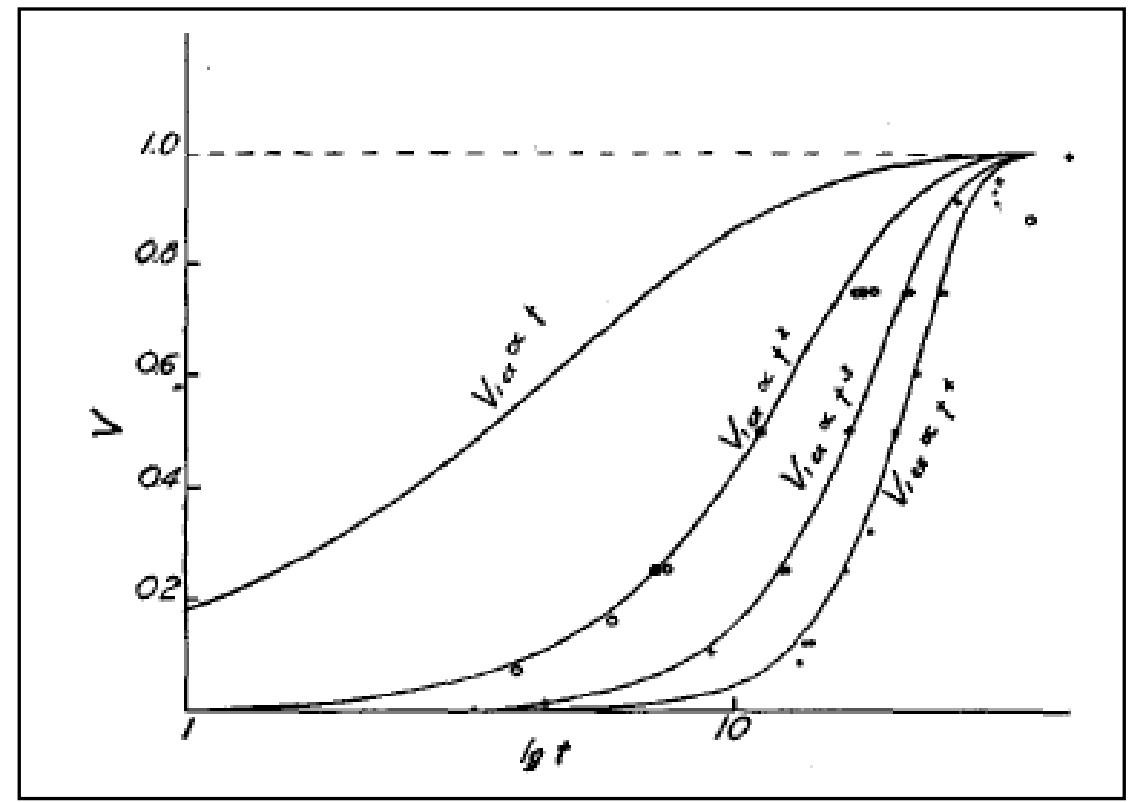

Figure 2.16: General shape of transformation curve on time dependence ${ }^{81}$.

From theoretical considerations ${ }^{80}$, the time dependence of the phase change is limited to $t^{4}$. The exponent is actually composed of two terms, $n=p+q$, where $p$ is either 0 or 1 based on heterogeneous or homogeneous nucleation, and $q$ represents the dimensionality of growth ${ }^{82}$. Finally, by taking the ratio of times required to achieve $75 \%$ and $25 \%$ phase transformation, we can estimate the shape of the crystals formed: 
Table 2.2: Dimensional estimates for crystalline patterns ${ }^{81}$.

$1.48 \leqslant \frac{t_{0.75}}{t_{0.25}}=\frac{\tau_{0.75}}{\tau_{0.25}} \leqslant 1.69$ for polyhedral growth.
$1.69 \leqslant \frac{t_{0.75}}{t_{0.25}} \quad \leqslant 2.2$ for plate-like growth.
$2.2 \leqslant \frac{t_{0.75}}{t_{0.25}}$

These are of course, estimates; this theory was developed about 75 years ago as an improvement over what was current knowledge. If one is concerned with crystalline shape, analysis with instruments such as XRD or polarized optical microscopy can give quantitative information. Some of the assumptions made to simplify the derivation introduce error into the results; for instance, Avrami noted that crystals that have been impinged upon in one growth direction likely do not immediately stop growth but may go in a different direction ${ }^{81}$. Furthermore, the dimensionality of growth should be limited only to integers but this is commonly not the case. For instance, Sun et al. reviewed much data for crystallization primarily in metals and found non-integer exponents that also shift as a function of composition and of non-isothermal crystallization $^{84}$. It seems that the genesis of the inconsistency is the sometimes faulty assumption of random distribution of seed nuclei. For an inhomogeneous distribution, crystallization occurs rather normally for low conversion, but as nuclei are exhausted the exponent tends to be lower. This is likely due to extensive impingement at high relative transformation, but Sun et al. have not offered any speculation as if this were the case.

It was previously stated that crystallization only occurs between the glass transition and melting temperature, due to chain and molecular mobility. Pantani et al., among others, have done experiments with PLA and other materials to determine the kinetics of crystallization processes ${ }^{85,86,87}$. Pantani et al. used a theory developed by Hoffman et al. ${ }^{88}$ to describe the effect of temperature on the kinetic parameter $k$ for PLA that had undergone various treatments. This expression was:

$$
k(T)=A_{1} \exp \left(\frac{-A_{2}}{T-T_{\infty}}\right) \exp \left(\frac{-A_{3}\left(T_{m}+T\right)}{2 T^{2}\left(T_{m}-T\right)}\right)
$$


Where the $A$ 's are experimentally determined constants and $T_{\infty}$ is a temperature roughly $30^{\circ} \mathrm{C}$ below the glass transition temperature ${ }^{85}$. All temperatures were reported in Kelvin. The fit of this curve was quite good with the collected data, shown in Figure 2.17:

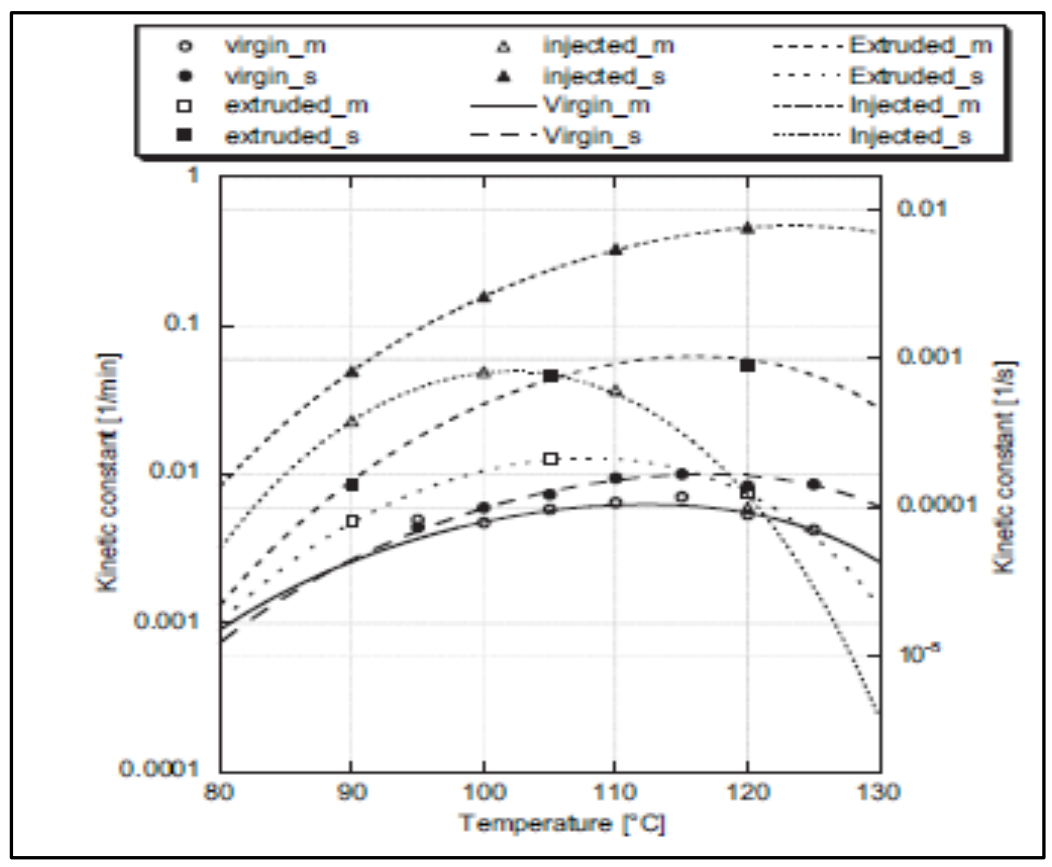

Figure 2.17: Curve fit of kinetic parameter for various PLA specimens ${ }^{85}$.

The materials were either virgin, extruded or injection molded PLA 2002D that had been isothermally crystallized from the molten or solid state. The kinetic constant in this work was calculated as the inverse of the time required for a sample to achieve 50\% of maximum crystallinity. They found that crystallization from the melt was slower than crystallization from the solid, and the extruded material was capable of the fastest crystallization. While there was no justification provided for the observation of faster crystallization from the glassy state, they explained that extruded samples experienced the most thermal degradation during processing, which created lower molecular weight fragments that could have acted as nucleation sites ${ }^{85}$. Data collected during this work can used for comparison of PLA-based materials.

\subsubsection{Crystallization of PLA-Based Materials.}

Because it is likely that PLA is unsuitable for durable use as a stand-alone material, it is more appropriate to review information on PLA-based blends. Because PLA is chiral, it may exist in either the $\mathrm{L}$ or $\mathrm{D}$ isomer, or can dimerize into a meso lactide. Kolstad studied formulations of 
PLA with varying amounts of meso compound and in some samples talc was added ${ }^{21}$. The crystallization half-time was found to increase by about $40 \%$ with the addition of every $1 \%$ of meso lactide, a severe retardation of the kinetics. In addition, he noted that the melting temperature could be reduced by up to $50^{\circ} \mathrm{C}$ which would cause difficulty for one wanting to create a PLA-based material with good thermal stability. The addition of talc was found to strongly nucleate crystallization ${ }^{21}$. In terms of nucleation, Kawamoto et al. used two different dibenzoylhydrazide agents at low loadings and found much faster crystallization rates and resultant improvement in mechanical properties, especially heat distortion temperature ${ }^{14}$.

Okamoto et al. blended several different low molecular weight plasticizers they referred to as polyester-diols with PLA and found them to increase the PLA crystallinity ${ }^{25}$. Unfortunately, the plasticizers made the blends less strong but more ductile. $\mathrm{Yu}$ et al. made PLA/aliphatic polycarbonate-diol copolymers and then performed chain extension using $\mathrm{HDI}^{26}$. This treatment gave the PLA chains a slightly higher melting temperature which indicated the formation of larger crystals, but these materials too were highly plasticized. For a more robust blend, Li and Shimizu fabricated compatibilized PLA/ABS materials which maintained most of the PLA crystallinity $^{28}$. While the blends lost stiffness, the addition of the ABS phase improved the ductility and imparted a large increase in impact strength. Xiao et al. created fully biodegradable blends of PLA/PBAT and found that a 40/60 blend had the maximum crystal growth rate (at the optimum annealing temperature) and crystallinity ${ }^{30}$. This point came with a change in the Avrami parameter, suggesting that once the phases inverted that crystal growth had been altered, perhaps due to different spatial dimensions of the different materials:

Table 2.3: Crystallization experiments with PLA/PBAT blends at $128^{\circ} \mathrm{C}^{30}$.

\begin{tabular}{|ccc|}
\hline PLA/PBAT & $n$ & $k\left(\mathrm{~min}^{-n}\right)$ \\
\hline $100 / 0$ & 2.5 & $5.5 \times 10^{-5}$ \\
$80 / 20$ & 2.5 & $7.2 \times 10^{-5}$ \\
$60 / 40$ & 2.4 & $2.4 \times 10^{-4}$ \\
$40 / 60$ & 2.0 & $7.0 \times 10^{-4}$ \\
\hline
\end{tabular}

One of the more interesting crystallization studies was that of Chen et al. who performed nonisothermal crystallization with PET/PLA blends ${ }^{34}$. They proposed that rather than considering 
the system as being a miscible two-phase blend, a three-phase blend in which mobile and rigid amorphous phases exist along with a crystalline phase. The mixtures exhibited a single glass transitions across the composition range, but the crystallization behavior was quite different. They found that PET could undergo crystallization in all circumstances, but if the crystallinity of the PET phase exceeded $10 \%$ the PLA would not crystallize:

Table 2.4: Composition and process dependent crystallization of PET/PLA blend ${ }^{34}$.

\begin{tabular}{|llllllll|}
\hline PET/PLA & $100 / 0$ & $90 / 10$ & $70 / 30$ & $50 / 50$ & $30 / 70$ & $10 / 90$ & $0 / 100$ \\
\hline$\phi_{\mathrm{C}}(\mathrm{PET})^{\mathrm{a}}( \pm .01)$ & 0.30 & 0.27 & 0.23 & 0.17 & 0.10 & 0.04 & 0 \\
$\phi_{\mathrm{C}}(\mathrm{PLA})^{\mathrm{b}}( \pm .01)$ & 0 & 0 & 0.01 & 0.10 & 0.18 & 0.22 & 0.25 \\
$\phi_{\mathrm{C}}(\mathrm{PET})^{\mathrm{c}}( \pm .01)$ & 0.31 & 0.25 & 0.20 & 0.14 & 0.08 & 0.03 & 0 \\
$\phi_{\mathrm{C}}(\mathrm{PL})^{\mathrm{d}}( \pm .01)$ & 0 & 0 & 0 & 0 & 0 & 0.02 & 0.25 \\
\hline
\end{tabular}

The explanation given for these unusual circumstances was that PET would form a much greater proportion of the rigid amorphous domain than PLA, which could impinge on space into which PLA might normally crystallize. Similarly strange results were obtained by Zhang et al. who experimented with PLA/PMMA blends in order to create durable materials ${ }^{74}$. Poly(D,Llactide)/PMMA blends were found to be miscible, but antagonistic, when prepared by solution/precipitation. If the same compositions were solution-cast, then intermediate compositions showed immiscibility. When PMMA was blended with PLLA and solution-cast, these blends had antagonism like the first blends, shown in Figure 2.18:

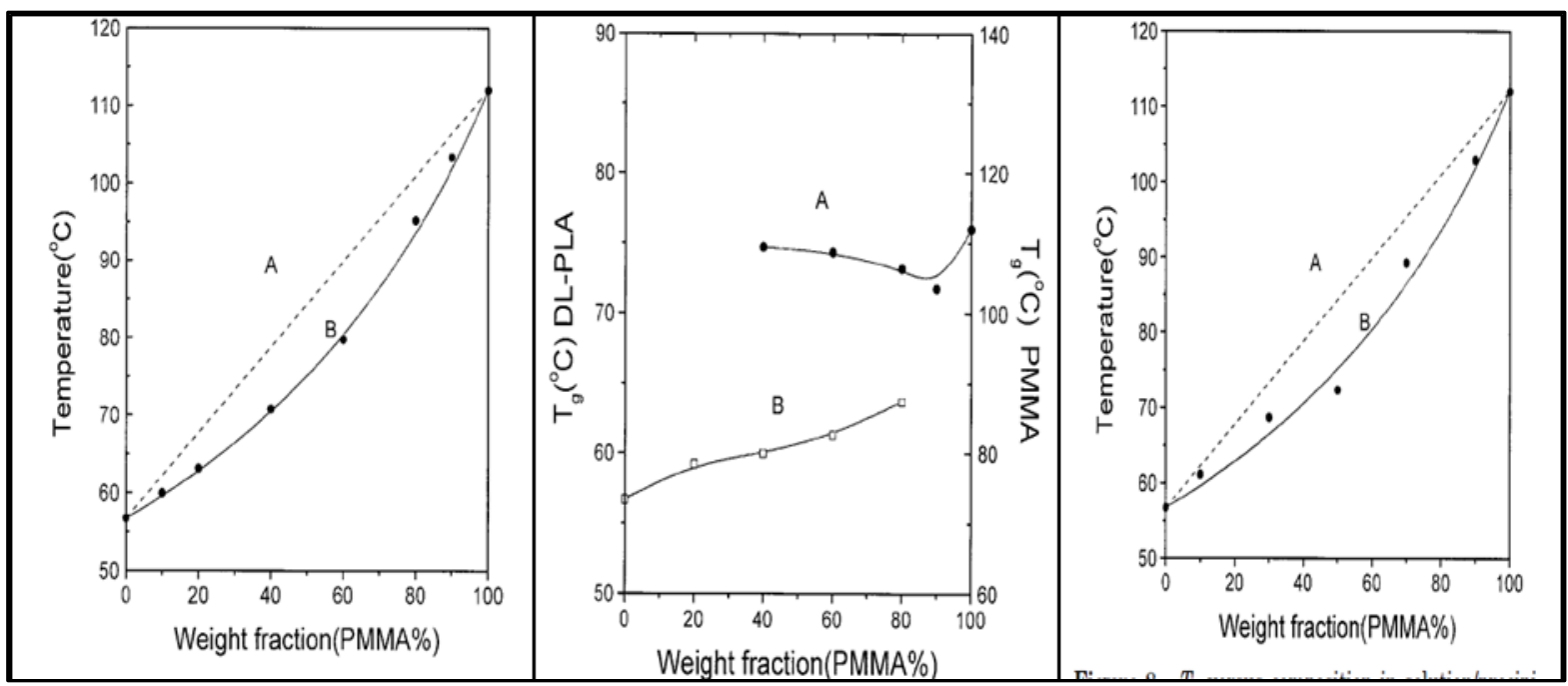

Figure 2.18: PLA crystallinity and blending affect miscibility of PLA/PMMA blends ${ }^{74}$. 
This behavior was suggested to have occurred due to the higher optical purity of the PLLA blends; these are much more crystalline than the practically amorphous poly(D,L-lactide). While the PLLA crystallization was hampered by the presence of PMMA as with the rigid amorphous PET/PLA blend above, there was still enough driving force created by the PLA to push the phases apart ${ }^{74}$. A final polymer blend for examination is also three phase, in this case the $\mathrm{PC} / \mathrm{PTT} / \mathrm{PBT}$ system $^{89}$. Both PTT and PBT are aromatic polyesters with fast crystallization rates, compared to the amorphous PC phase. The blend studied was 25/25/50 PTT/PBT/PC by weight and was prepared by twin-screw extrusion, and PC was found to retard the crystallization of the other phases, perhaps due to interference with chain rearrangement due to its bulkiness ${ }^{89}$.

By examining the available literature addressing the lacking properties of PLA and PLA-based blends, it should be possible to formulate solutions to them. Therefore, we planned to examine the effects of PLA crystallinity, blending/encapsulating PLA with the more stable PC phase and introducing graphene into the composites as a barrier material to improve mechanical properties and hydrolytic stability. 


\section{CHAPTER 3}

\section{MATERIALS AND EXPERIMENTAL PROCEDURES}

\subsection{Materials.}

Polymeric materials for this study were obtained from the manufacturers and processed without further purification. Polycarbonate was provided from Bayer MaterialScience in the form of Makrolon 2608, a transparent FDA-approved medical grade polycarbonate often used for cardiovascular devices, inhalers, and catheters. Polylactic acid was purchased from NatureWorks in the form of PLA 2002D, an extrusion grade used alone or in formulations for food packaging and serviceware applications. To test the effect of fillers with enhanced barrier properties, graphene nano-platelets $\mathrm{xGnP}-\mathrm{H}$ from XG Sciences were employed. This additive consists of graphite sheets with nominal thickness of $12-15 \mathrm{~nm}$ and width $25 \mu \mathrm{m}$, with a surface area of $60-80 \mathrm{~m}^{2} / \mathrm{g}$. No additional treatments were performed on the graphene additive. The PC, PLA and graphene were then blended together in compositions ranging from pure PC to pure PLA in 20 weight $\%$ increments. If graphene particles were added, the amount was kept constant at 2 weight \%; for instance, an unfilled system of 80/20 PC/PLA would translate to 79/19/2 when graphene filled. The resultant blends were then used directly in pellet form for crystallization experiments, melt flow analysis or for hydrolytic degradation, or compression molded into ASTM standard sized pieces for mechanical testing and into plates used for viscosity and solution degradation tests. The remainder of this section will outline how each mode of processing or data collection was undertaken.

\subsection{Experimental Procedures.}

\subsubsection{Compounding of the Polycarbonate and Polylactic Acid.}

To create the PC/PLA blended polymers, the WVU Leistritz Micro-27 twin screw extruder (TSE) was utilized. It is shown here with ancillary equipment in Figure 3.1: 


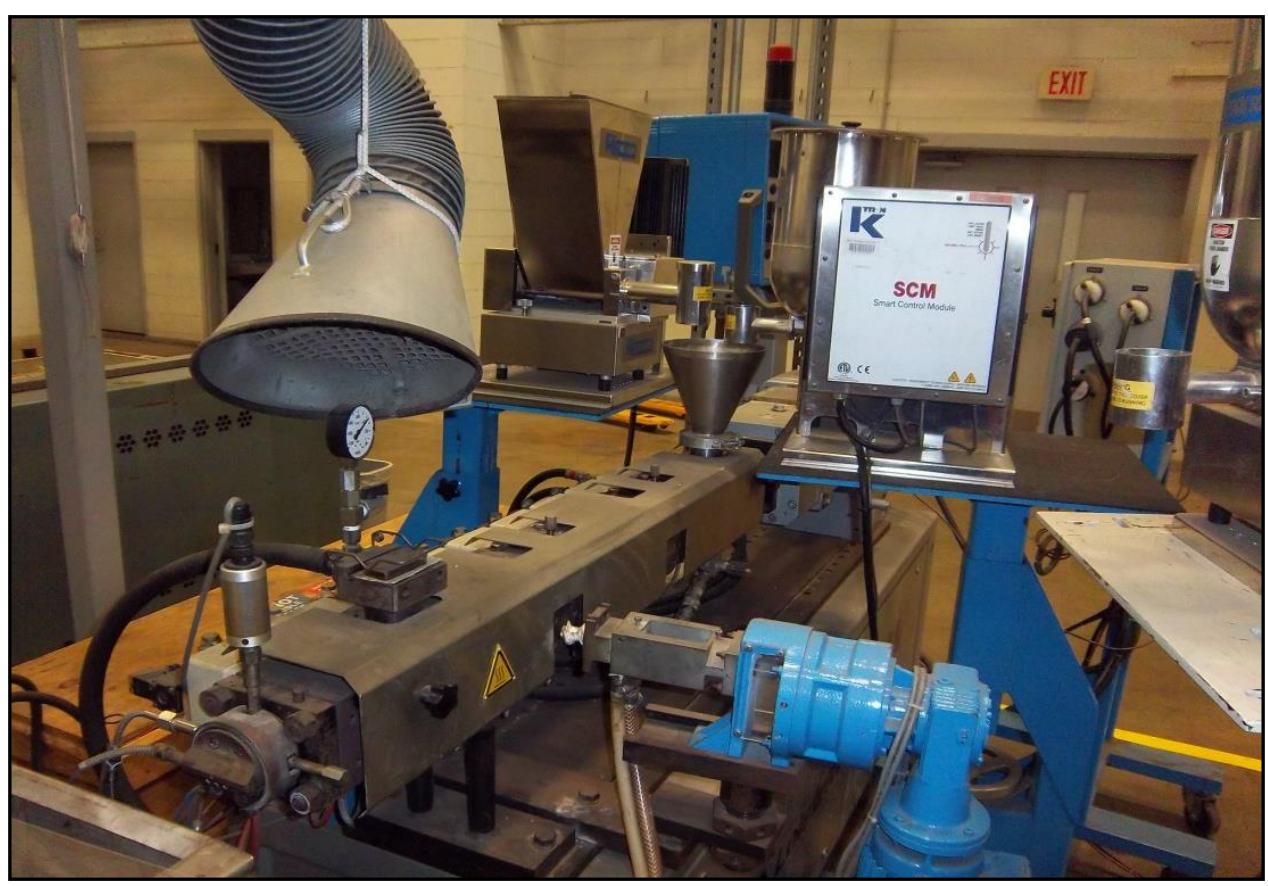

Figure 3.1: Leistritz 27 TSE with feeders, control box and vacuum trunk.

Prior to extrusion, the polymers were thoroughly dried to remove residual moisture which would cause degradation and molecular weight loss. Polycarbonate was dried at $120^{\circ} \mathrm{C}$ in a standard oven without convection for at least 4 hours while PLA was dried at $90^{\circ} \mathrm{C}$ for 4 hours, as recommended on manufacturer's data sheets. The machine was then operated in co-rotational mode, with constant temperature profile across all blend compositions. The thermal profile was selected based on advice from Bayer MaterialScience, and is a compromise between thermal degradation of the PLA and softening PC to a degree that allows adequate blending. The profile used for the blends, from feed hopper to die, is 100-175-230-230-230-240-240-240-240-250 ${ }^{\circ}$. A vacuum of about 400 Torr was imposed near the extruder die in order to remove any possible moisture that remained in the melt. In order to achieve a constant but significant screw torque, vital to blending materials with a large viscosity ratio, the remaining extrusion parameters were varied. The following combination of variables was used to maintain a screw torque of roughly 70 percent of maximum: 
Table 3.1: Further extrusion parameters.

\begin{tabular}{|c|c|c|c|c|c|}
\hline Sample & $\%$ PLA (wt.) & $\%$ PC (wt.) & $\dot{m}(\mathrm{~kg} / \mathrm{hr})$ & Screw speed $\Omega$ (rpm) & Die $\Delta P(p s i)$ \\
\hline $100 N T$ & 100 & 0 & 11 & 110 & 360 \\
\hline $80 N T$ & 80 & 20 & 11 & 125 & 400 \\
\hline $60 N T$ & 60 & 40 & 11 & 150 & 480 \\
\hline $40 N T$ & 40 & 60 & 10 & 150 & 530 \\
\hline $30 N T$ & 30 & 70 & 8 & 150 & 580 \\
\hline $20 N T$ & 20 & 80 & 8 & 150 & 660 \\
\hline ONT & 0 & 100 & 4 & 150 & 600 \\
\hline
\end{tabular}

The sample names describe the PLA content by weight and the suffix $N T$ means no subsequent treatment has been performed; this will be expounded upon later. By adding food coloring to the samples of neat PLA and PC, the residence time was estimated to be between about 1-6 minutes depending on exact conditions. The extrusion system was operated using a KSL Smart Line Control device, which oversaw two feed units, the K-Tron Soders K-CL-24-KQx4 and K-CL-24KT20. After extrusion, the molten strands of polymer were collected on aluminum trays and left to cool to solidification in ambient conditions. Conventionally, extrudate is passed through a water bath for cooling and then cut into pellets; however, exposing PC and PLA in the melt to $\mathrm{H}_{2} \mathrm{O}$ can cause de-polymerization and loss of molecular weight. After these large blocks had cooled overnight, they were then fed into the Econogrind ESL180/430 for pelletizing. As with all operations, proper safety attire such as steel toed shoes, heavy gloves and eyewear were worn during use. After granulation, the pellets were collected in labeled bags for storage. No postcollection drying is necessary as all operations were carried out in the absence of moisture save for atmospheric conditions.

\subsubsection{Compounding of the Polycarbonate and Polylactic Acid with Graphene.}

While use of twin screw extrusion is extremely common for industrial scale production of all varieties of plastics, it is not the best option for lab scale formulation of the graphene-containing materials. It is quite difficult to maintain consistent feeding of minute amounts of additives, especially since graphene is a light, fluffy powder which is prone to adhering to surfaces. Therefore, blends containing graphene were created using a Haake PolyDrive internal mixer in small batches. Prior to blending, the PC and PLA were dried as above, with graphene dried using the same conditions as the polycarbonate. The mixer is operated by accompanying software, and 50 gram samples of the appropriate compositions were mixed at $240^{\circ} \mathrm{C}$ for 5 
minutes with roller rotor speed of $80 \mathrm{rpm}$ as the standard condition. The machine can be seen in Figure 3.2 as follows and compared in size to the much larger TSE:

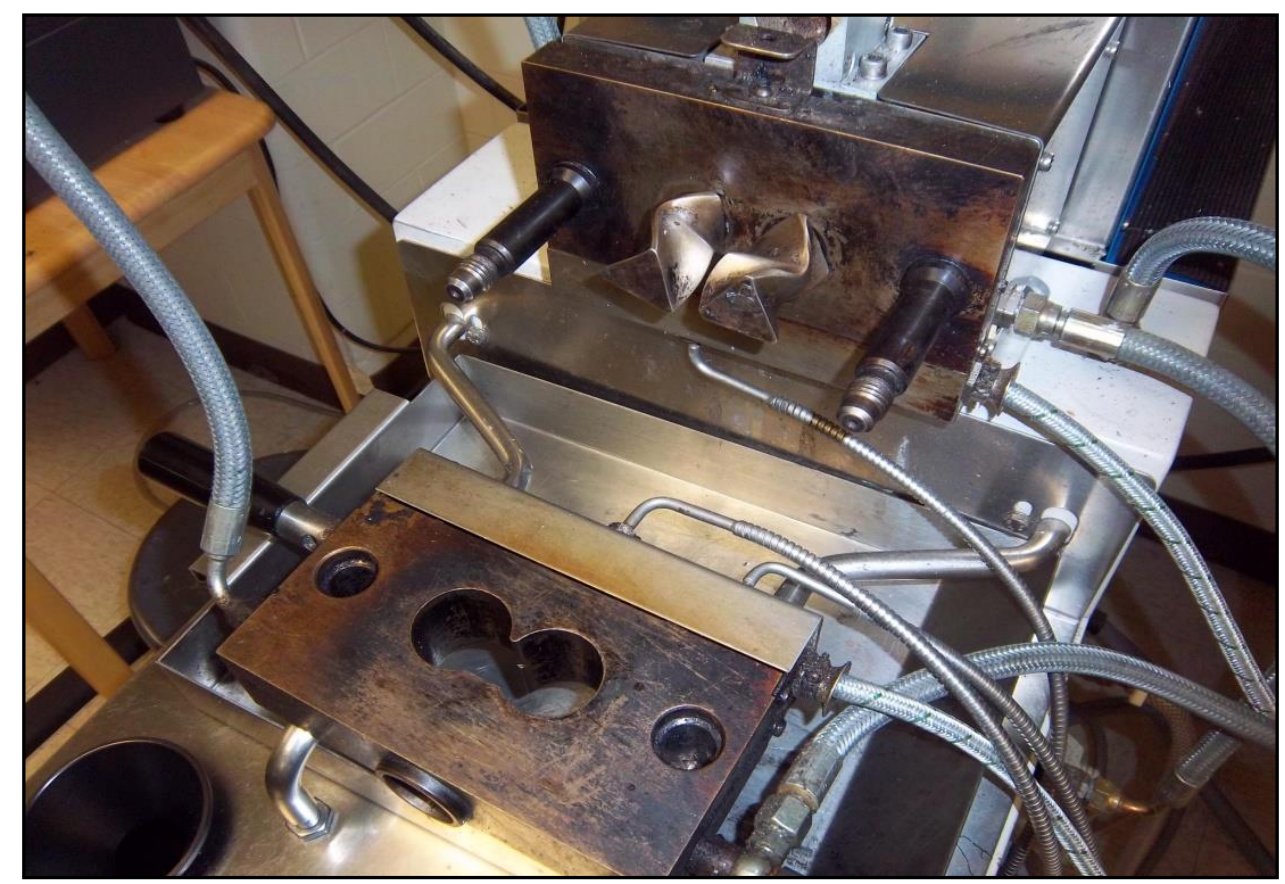

Figure 3.2: Internal mixer showing roller rotor blades.

Upon completion of mixing, the molten blends were scraped from the components of the mixer and collected, and the mixer vigorously cleaned with brass implements. Because sample sizes from the mixer were much smaller than those from the extruder, the bench scale Dynisco Mini granulator was used to create pellets. The samples were then collected into labeled bags as before.

\subsubsection{Compression Molding.}

While the pellets were used for hydrolytic testing and part of the DSC evaluation, molded parts were required for flexural property and some degradation tests. The PHI SQ-230H compression molder was utilized to create these pieces, shown in Figure 3.3: 


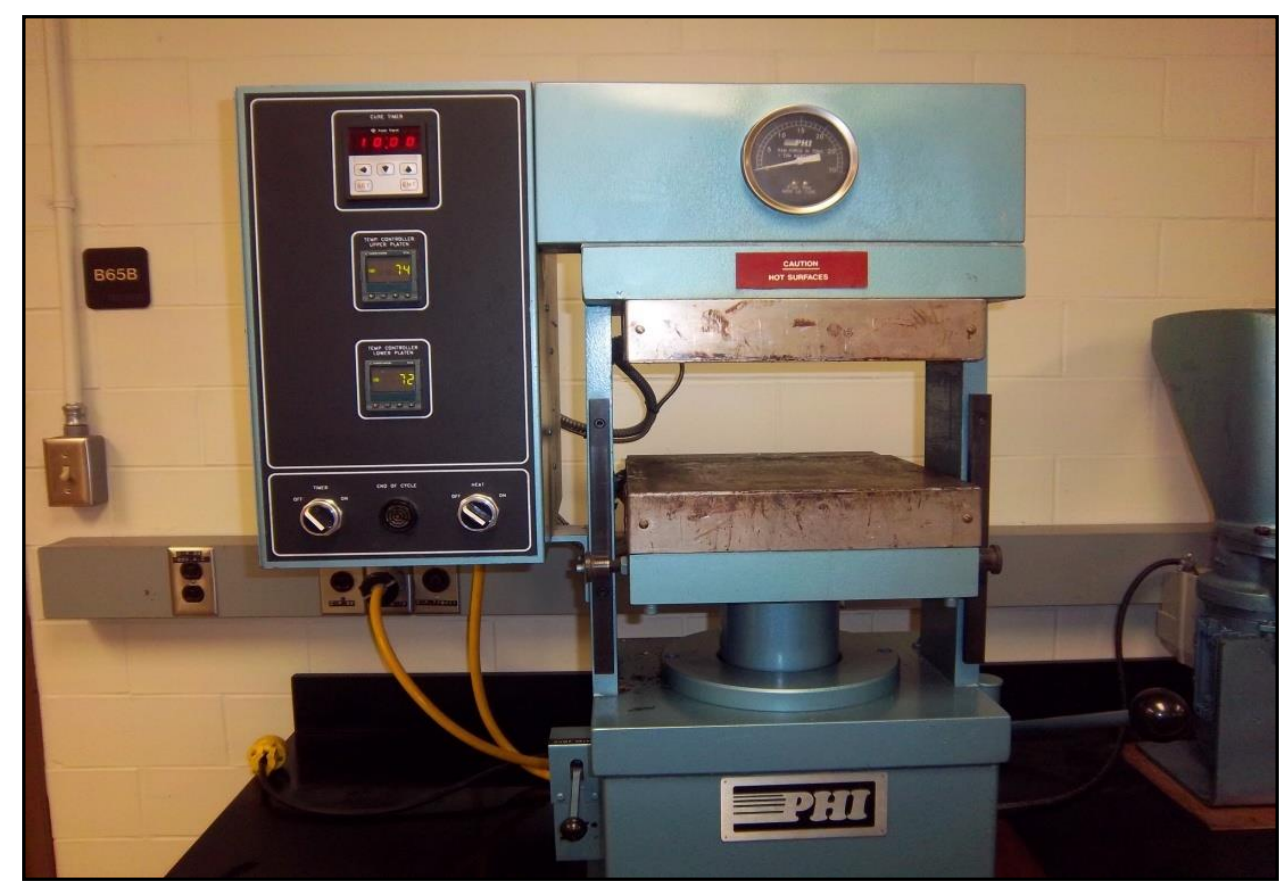

Figure 3.3: Compression molder in open position.

Pellets dried in the manner above were molded at $225^{\circ} \mathrm{C}$ between aluminum foil sheets coated with PTFE-based mold release for 10 minutes under a force of 10 tons. For degradation testing a parallelepiped of width and length $127 \mathrm{~mm}$ and $3 \mathrm{~mm}$ thickness was molded. After removal from the machine, the panel was left to cool fully in ambient lab conditions. Next, each individual panel was cut into smaller squares of approximately $30 \mathrm{~mm}$ size using a band saw. These were the pieces for degradation and rheological tests. Flexural bars were created at the same conditions using 7 cavity wells, producing pieces that were roughly $127 \mathrm{~mm}$ long and 12.5 mm wide. Two sets of molds having either $1 / 10^{\text {th }}$ or $3 / 16^{\text {th }}$ inches nominal thickness were used to investigate the effect of part thickness on hydrolytic stability.

\subsection{Data Collection and Analysis.}

\subsubsection{Melt Flow Index.}

A simple yet powerful tool for quality control of polymeric materials is the melt flow index or MFI, performed according to ASTM D1238. In our laboratory, the Dynisco LMI Series 4000 Melt Indexer was used to carry out melt flow experiments in a manner similar to a capillary rheometer. It is shown here in Figure 3.4: 


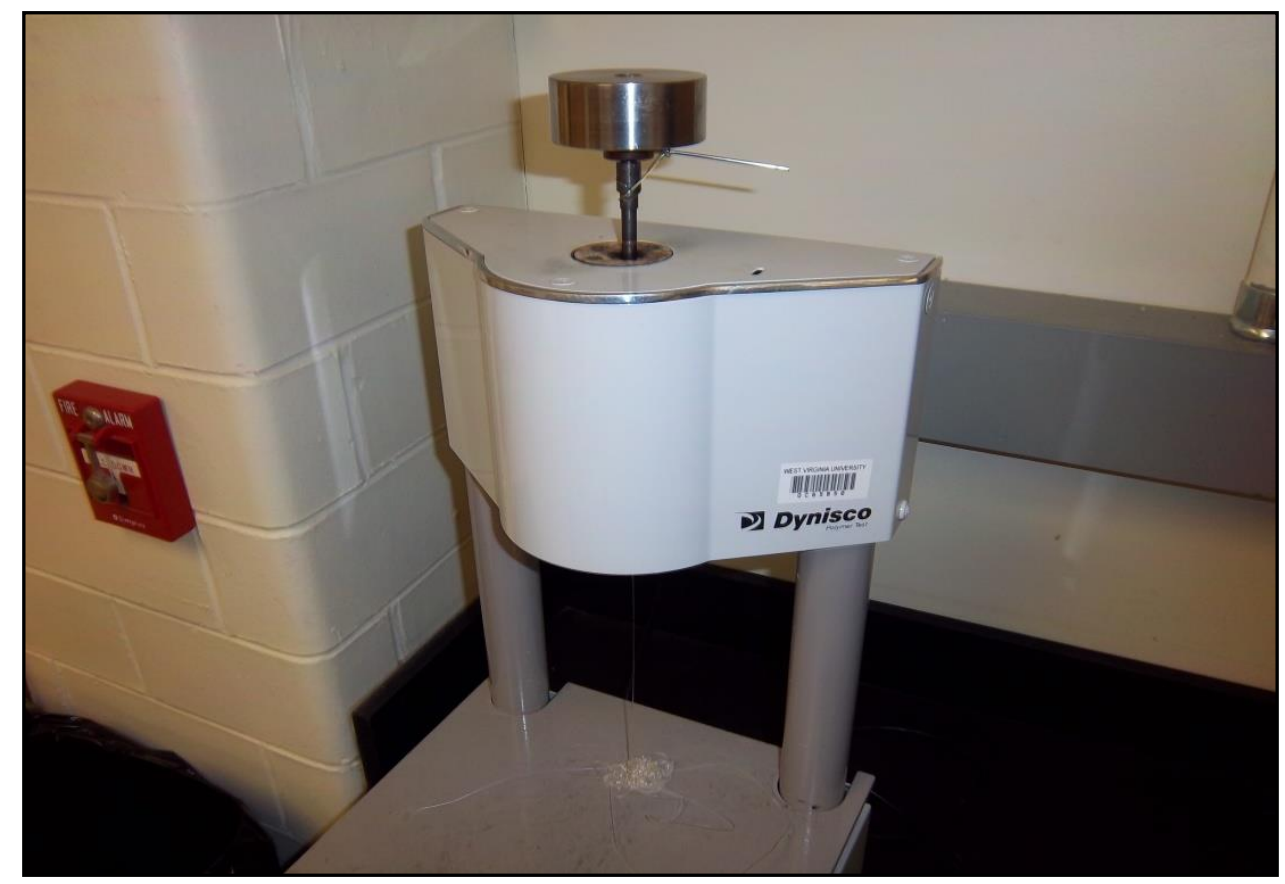

Figure 3.4: Melt flow indexer with piston and weight during test.

One charges a small mass ( 4-5 g) of dried pellets into the heated barrel of the indexer, and allows the material to melt. A load in the form of a piston and weight is added at the outset or during this melting period such that the test is ready to begin after $7 \pm 0.5$ minutes. Material then flows from the bottom orifice, which is collected while a demarcated region on the piston plunger is inside the capillary barrel. The mass of extrudate and time to collect are used to convert the flow rate into the specified units of grams per 10 minutes. While the technique is used to measure a flow rate, it is a proxy for the molecular weight and viscosity. If a significant change in MFI is noted, one should expect that physical property changes will occur in the pellets or a subsequently molded part. For accuracy and repeatability the testing was performed in triplicate at minimum.

\subsubsection{Mechanical Testing.}

The flexural specimens obtained from the compression molder were allowed to rest for at least 40 hours and then were subjected to testing. An Instron 5567 machine was used to obtain mechanical properties in accordance with ASTM D790. Bars underwent the three point bending experiment with a cross head speed of $1.387 \mathrm{~mm} / \mathrm{min}$, and were performed at least 5 times for accuracy and repeatability. 


\subsubsection{Differential Scanning Calorimetry.}

To determine the crystalline and thermal properties of the blends, DSC was used in two modes; a "first heat" mode for basic thermal properties and the Avrami kinetic mode for crystallization behavior. Each was performed using 6-9 mg samples pressed into aluminum pans in the TA Instruments Q100 DSC under nitrogen and helium flow. The first heat procedure is given:

- Load the sample into the DSC at $25^{\circ} \mathrm{C}$.

- Cool $10^{\circ} \mathrm{C} / \mathrm{min}$ to $10^{\circ} \mathrm{C}$ and remain isothermal for 1 minute.

- Heat $10^{\circ} \mathrm{C} /$ min to $250^{\circ} \mathrm{C}$ and remain for 5 minutes.

- $\mathrm{Cool} 10^{\circ} \mathrm{C} / \mathrm{min}$ to $25^{\circ} \mathrm{C}$.

From this experiment, the glass transition(s) and any residual crystallinity from previous processing could be evaluated. Furthermore, this procedure was used to evaluate selected degraded and aged samples to find the effect of those processes on the thermal properties. The Avrami experiments are isothermal annealing tests. Using the same sample sizes and conditions as before, these tests were performed on both unfilled and graphene containing pellets.. The test procedure is as follows:

- Load the sample into the DSC at $25^{\circ} \mathrm{C}$.

- $\mathrm{Cool} 10^{\circ} \mathrm{C} / \mathrm{min}$ to $10^{\circ} \mathrm{C}$ and remain isothermal for 1 minute.

- Heat at $10^{\circ} \mathrm{C} / \mathrm{min}$ to $250^{\circ} \mathrm{C}$ to destroy any present crystals $\left(\mathrm{T}_{\mathrm{m} . \text {.eq,PLA }} \sim 210^{\circ} \mathrm{C}^{14,90}\right)$.

- Remain isothermal for 5 minutes.

- Cool at $40^{\circ} \mathrm{C} / \mathrm{min}$ to the desired crystallization temperature $\mathrm{T}_{\mathrm{c}}$.

- Remain at $\mathrm{T}_{\mathrm{c}}$ for 3 hours.

- Melt again at $10^{\circ} \mathrm{C} / \mathrm{min}$ to $250^{\circ} \mathrm{C}$, cool to room temperature and remove sample.

This procedure gives information regarding time dependent crystallization from the melt, which is important considering that industrial processes such as injection molding cool from the melt. This series of experiments is similar to that of Pantani et al. who studied the crystallization behavior of pure PLA that was processed in a variety of ways ${ }^{85}$. These tests were performed on all blends except those without any PLA content, as PC is known to be fully amorphous under typical circumstances. As a result, the crystallinity given for any calculation is based on the weight fraction of PLA. For example, for an annealing experiment performed on a 60/40 PC/PLA blend, the actual DSC value is divided by 0.4 to give the proper result. 


\subsubsection{Parallel Plate Rheology.}

Rheological measurements give an indication of how molten polymer will flow under varying conditions. The Rheometrics RMS-800 rheometer with parallel plate fixture coupled to the associated RSI Orchestrator software package was used. A $25.0 \mathrm{~mm}$ diameter plate was used at a gap set to $1.4 \mathrm{~mm}$. Samples were inserted into the rheometer, melted, and tests begun within 10 minutes to reduce the chances for thermal degradation. To collect the data, a sample was loaded and tested to completion three times consecutively. Each test takes roughly 10 minutes to sweep from 0.1 to 100 radians per second, meaning one polymer sample experiences slightly less than 40 minutes in the rheometer. Tests were performed using $12 \%$ strain to assure the results were in the linear viscoelastic region for both materials.

\subsubsection{Degradation in Acid/Base Media.}

Part of the appeal of PLA is that it is a biodegradable material. In Chapter 2, we detailed the many pertinent variables and phenomena that are involved in the dissolution of PLA. While polycarbonate shares some chemical characteristics with polylactic acid, it is much more resilient in the face of hydrolytic or similar attack. Here, we desire to evaluate the impact of degradation media and temperature on the materials. The conditions of the degradation tests are:

Table 3.2: Solution conditions and times for degradation tests.

\begin{tabular}{|c|c|}
\hline Condition & Values \\
\hline Temperature & $50^{\circ} \mathrm{C}$ \\
\hline $\mathrm{pH}$ & $4.0,7,10.0$ \\
\hline Exposure Time & $7,14,21,42$ days \\
\hline
\end{tabular}

The media were prepared in a manner similar to that of Ginde et al. where the acid or alkali was prepared as 0.25 molar buffer solution ${ }^{91}$. The degradation temperature was chosen as $50^{\circ} \mathrm{C}$. The acid solution was the acetic acid/sodium trihydrate acetate system, while the base was $\mathrm{Na}_{2} \mathrm{CO}_{3} / \mathrm{NaHCO}_{3}$. Both of these are commonly encountered in biological studies, which are appropriate given the nature of PLA. Neutral media of $\mathrm{pH} 7$ is de-ionized water, changed out weekly in order to prevent $\mathrm{pH}$ swings due to formation and dissolution of acidic or basic breakdown by-products. Further, the acid and base samples were tested weekly for $\mathrm{pH}$ drift and corrected by titration if necessary. Samples were placed in $125 \mathrm{ml}$ glass jars and checked for mass loss at each time interval. After removal from the jars, samples were washed clean with 
distilled water and vacuum dried at room temperature to remove any residual solution. Each week, pieces are collected for morphological, crystalline, and viscosity measurements.

\subsubsection{Hydrolytic Stability.}

In addition to testing the effects of liquid solvents of differing $\mathrm{pH}$, a practice for evaluating the degradation resistance of plastics is exposure to high temperature and high humidity. This test is inspired by ASTM D7444 for heat and humidity aging of oxidatively degradable plastics. Large Pyrex desiccator jars are sealed into a watertight unit by smearing a thin layer of silicone grease on the lip of both the jar and lid. They are then filled with approximately $500 \mathrm{~mL}$ of deionized water and placed into an oven with good temperature control. After coming to equilibrium, either pellets or mechanical testing pieces are introduced into the jar and resealed. The pots are held at $50^{\circ} \mathrm{C}$ and the duration of exposure is varied during this testing. According to workers at Ford, samples that are aged for 1 week at $50^{\circ} \mathrm{C} / 90 \% \mathrm{RH}$ experience the equivalent of 2 months outdoor aging in Florida ${ }^{63}$. When testing is complete, the samples are evacuated into a room temperature vacuum oven (also containing desiccant) to remove moisture without altering the thermal properties. Pellets are then subjected to DSC study for evaluation of thermal and crystalline properties, as well as MFI to determine a loss in viscosity or mechanical properties. It is known from preliminary results that negligible weight change occurs during exposure. Flexural bars are tested in this manner to ascertain the loss of mechanical properties during exposure. However, a standard does not exist for this particular type of degradation experiment. ASTM D3826 gives the brittle point for degradation of polyethylene and polypropylene as "the point in the history of a material when $75 \%$ of the specimens tested have a tensile elongation at break of $5 \%$ or less ${ }^{92}$, but this is not an equivalent test. Harris performed experiments very similar to those of this work and simply reported a point by which severe property damage or

loss had occurred ${ }^{63}$. Therefore, we will do as Harris did and deem that materials with obviously severe loss of mechanical properties are unfit for durable application; it would be up to a product designer with a specific target application to propose a criterion for unacceptable degradation.

\subsubsection{Morphological Studies.}

To probe the small scale structure of the blended material, the Hitachi S-4700 Scanning Electron Microscope (SEM) was operated by WVU staff. All samples were first cryogenically fractured using liquid $\mathrm{N}_{2}$ in order to preserve the morphology. They were then sputter coated with an 
extremely thin layer of gold, necessary for non-conductive specimens such as plastics and ceramics.

Using all of the above techniques, we can then evaluate the initial and final properties of the composites and blends to determine what set or combination of treatment provides a material with mechanical and physical integrity sufficient for durable applications. 


\section{CHAPTER 4}

\section{RESULTS AND DISCUSSION}

\subsection{Introduction to Results and Discussion.}

By using the techniques listed in Section 1.4, an evaluation of both compositions and available treatments was carried out in order to make materials that would contain both significant biobased content and be durable enough for exposure to harsh conditions. The materials tested ranged from $0-100 \%$ PLA phase with the possibility of no treatment, annealing to increase crystallinity, the inclusion of a small graphene loading to provide a barrier to diffusion, and in some cases both annealing and graphene. Blends that were PC-rich in which the PLA was fully encapsulated by PC were internally mixed with the goal of altering the interfacial morphology so as to reduce the diffusive flux of an attacking species into the PLA phase. There was also a study on the effect of part thickness on retarding diffusion, ranging from small polymer pellets up to molded parts with thicknesses of about $4 \mathrm{~mm}$. These were tested in a variety of ways to collect initial mechanical, thermal and flow properties, then subsequently degraded either by solvent or in a heat/humidity chamber up to a maximum period of 1 year equivalent outdoor exposure. The task is then to determine what combination of formulation and treatment options works best to provide a material suitable for use in durable goods with lifecycles long enough for use in valuable sectors such as consumer or commercial electronics or automotive.

The results will be presented in the order of objectives listed in Section 1.4 to evaluate the outcomes of the least complex procedures first.

\subsection{Effect of Untreated vs. Treatments of PLA Polymer.}

We first evaluate PLA polymer with and without any subsequent treatments to determine the stability of this material. The PLA specimens examined in this research are listed in Table 4.1: 
Table 4.1 PLA experimental materials and method of production.

\begin{tabular}{|l|r|r|r|l|c|}
\hline Sample & \%PLA & \%PC & \% Graphene & Method & Mixing Conditions \\
\hline $100 N T$ & 100 & 0 & 0 & extrusion & see Table 3.1 \\
\hline $100 A$ & 100 & 0 & 0 & extrusion & see Table 3.1 \\
\hline $100 G$ & 98 & 0 & 2 & int mix & $240^{\circ} \mathrm{C}-80 \mathrm{rpm}-5 \mathrm{~min}$ \\
\hline
\end{tabular}

In this and all subsequent nomenclature, $N T$ is for no treatment, $A$ is for annealed and $G$ for graphene containing. The number preceding the lettering gives the nominal PLA content in weight percentage. The extrusion conditions are not given in this Table; rather they are listed previously in Chapter 3 as these are not identical across all compositions.

The structure of each of the samples was investigated by SEM as described in Chapter 3 to evaluate the morphology and dispersion of the blends. Each of the images comes from a cryofractured plaque that had been compression molded at $225^{\circ} \mathrm{C}$ for 10 minutes under 10 tons force; examination of the extruded or mixed pellets was infeasible due to small size and obvious artifacts of processing. No annealed samples were imaged as it was deemed unlikely that heat treatment should affect the morphologies of the blends. The surfaces shown in Figure 4.1 are the internal cross sections of unfilled (a) and graphene-containing (b) PLA plaques:
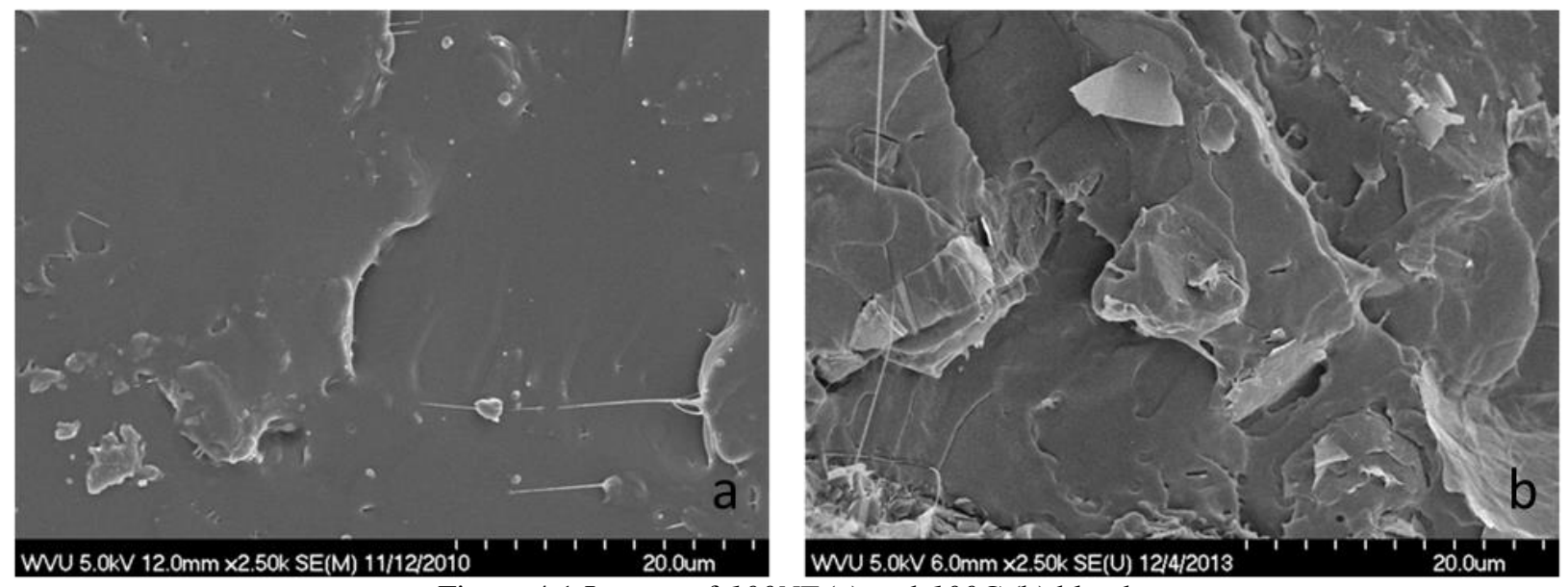

Figure 4.1:Images of 100NT (a) and 100G (b) blends.

Then, in Figure 4.2 there is a comparison of an untreated square compared to an annealed panel to verify this assertion: 

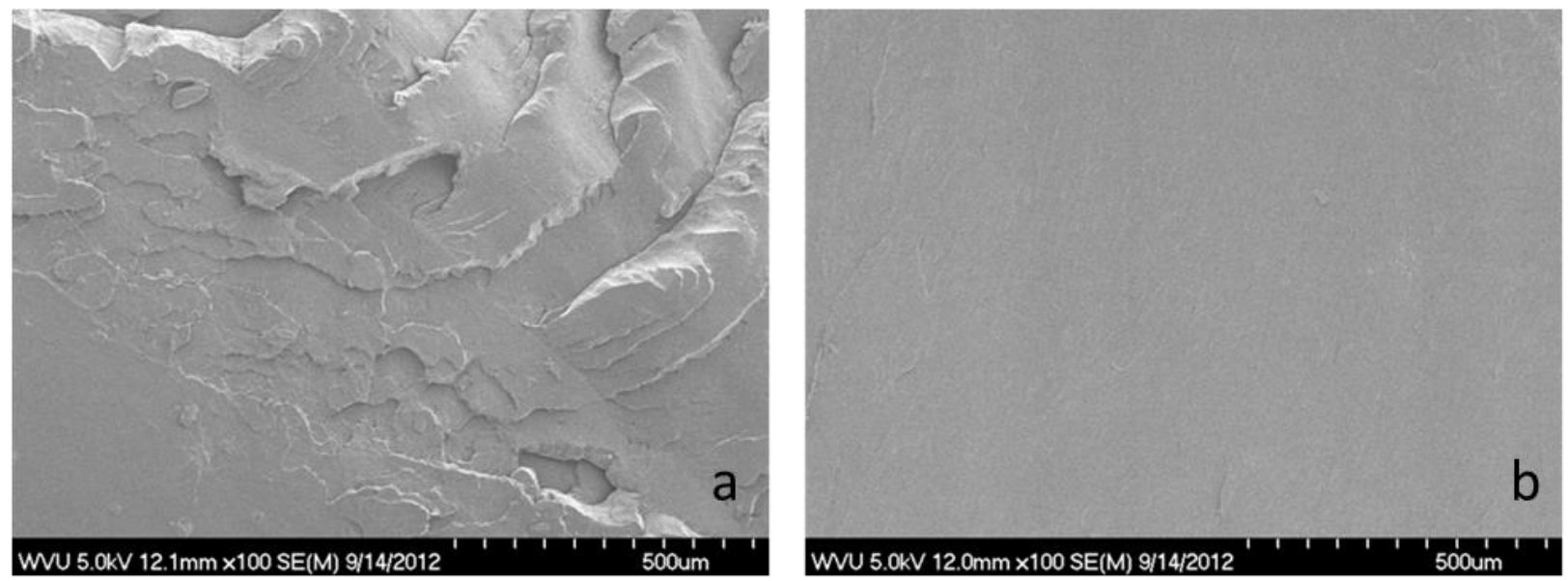

Figure 4.2: Images of amorphous (a) 100\% PLA square contrasted with annealed (b) 100\% PLA square.

At most, the untreated square actually appears to have fractured in a more ductile manner than the annealed sample; mechanical testing will later show this to be true. In Figure 4.1(b), there are large, rough graphene plates protruding in and out of the image; it is known that total exfoliation and dispersion of nano-scale materials is difficult to achieve by conventional processing methods (e.g. ${ }^{93}$ ) Particle size analysis is not necessary for these images and will be addressed later. The next step is to address the annealing process itself and address the crystallization kinetics before finally comparing the effect of these treatments on the hydrolytic stability of the PLA formulations.

\subsubsection{Crystallization of the Extruded PLA and PLA/Graphene Composite.}

The procedure for performing time-dependent crystallization experiments was given in Section 3.3.3. Raw data from the DSC experiments is used for calculation of the crystallization kinetics. The linked Universal Analysis software is used to plot the heat flow into the sample to obtain the crystallinity, as in Figure 4.3: 


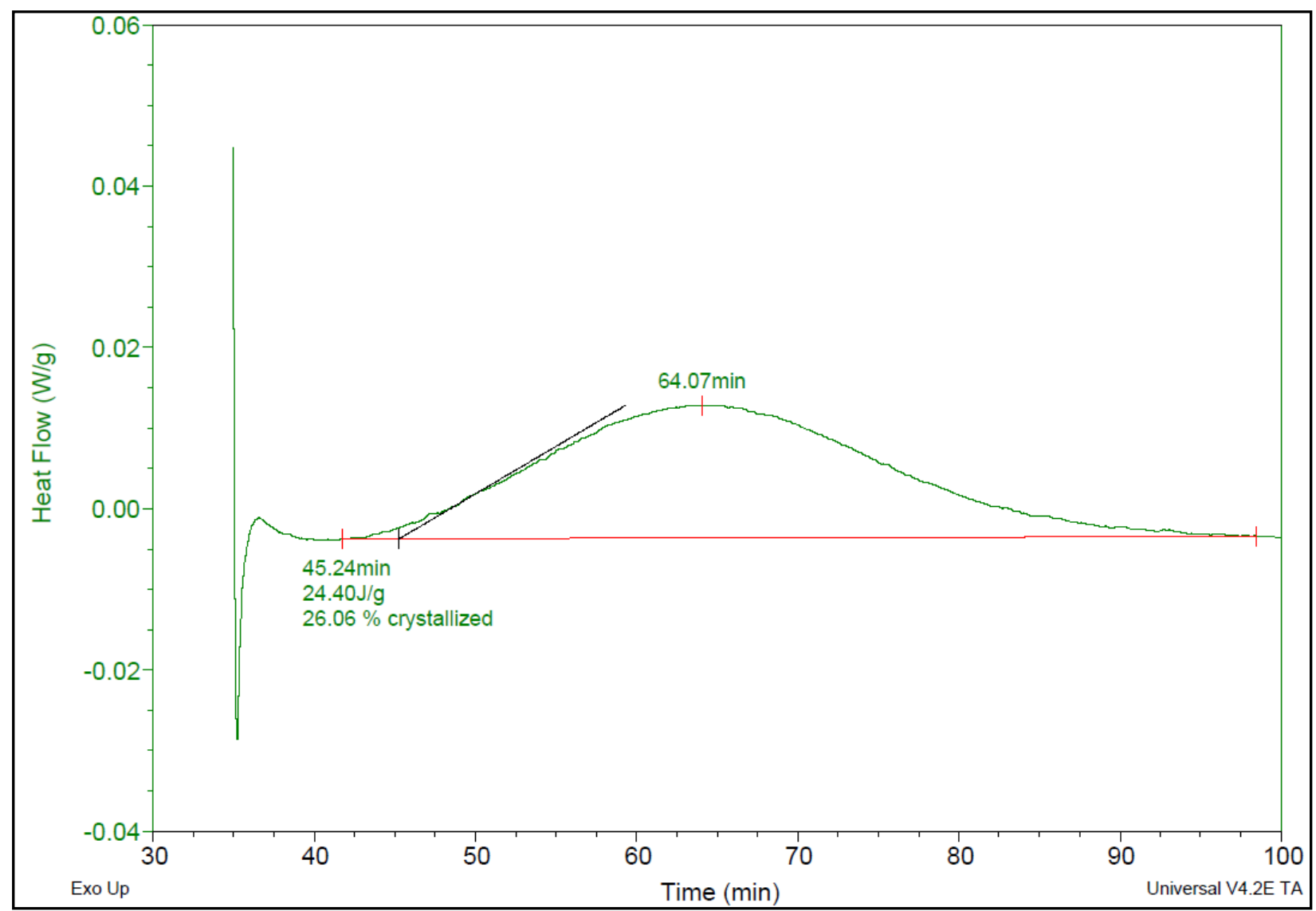

Figure 4.3: Heat flow from sample during isothermal crystallization experiment.

This sample was $100 \%$ PLA annealed at $90^{\circ} \mathrm{C}$ without the addition of graphene. It must also be mentioned that the above case shows a flat baseline of integration; this is not always the case and then it must be corrected for. The value from the software is then used to guide the numerical integration of this curve to obtain the characteristic S-shaped time-transformation curves that were shown as Figure 2.16. Any method such as the trapezoid rule or Simpson's rule may be utilized. For instance, the time-transformation curve generated from the data in Figure 4.3 is plotted in Figure 4.4: 


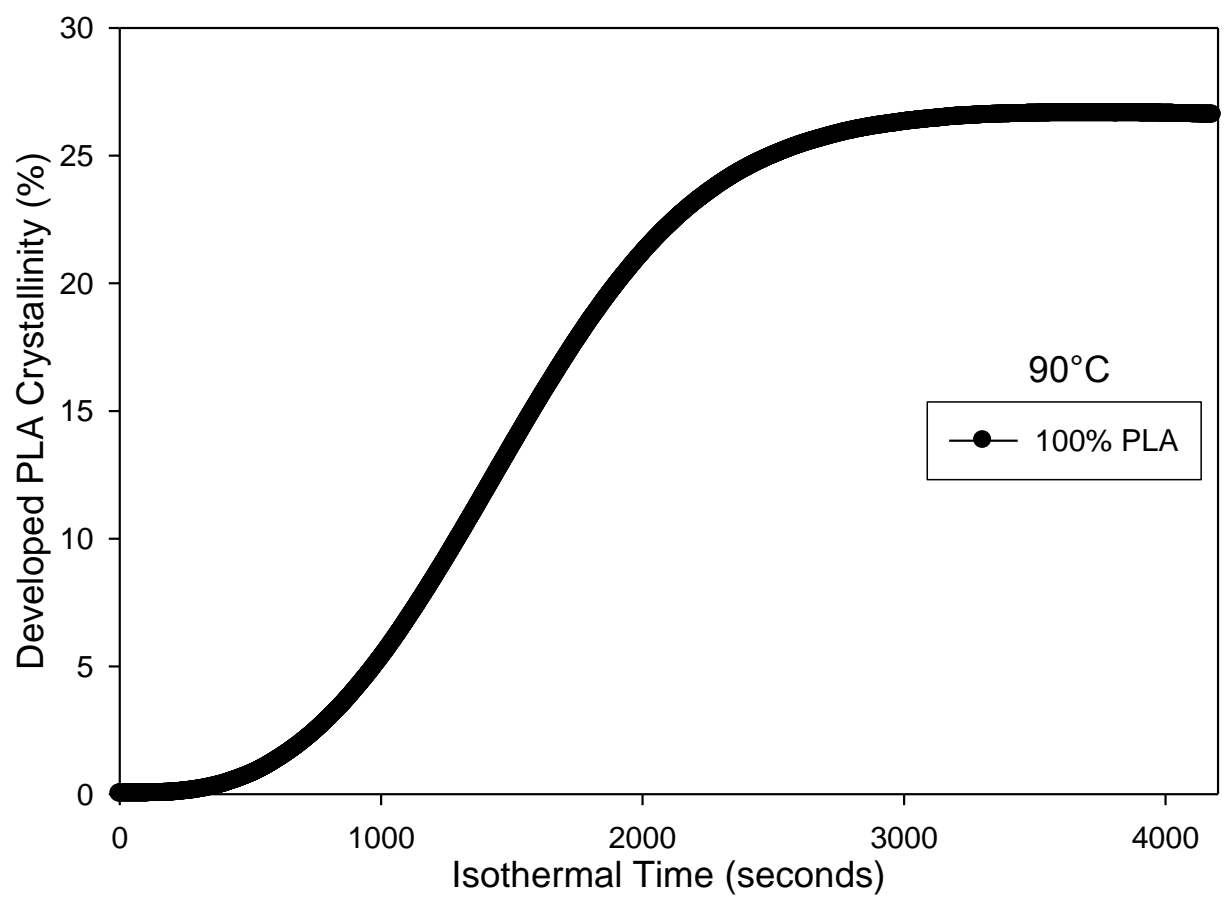

Figure 4.4: Time-transformation curve for the data presented in Figure 4.3.

Here the calculation must take into account the published infinite heat of fusion of PLA (93.6 $\mathrm{J} / \mathrm{g}$ ) and the weight equivalent of PLA must be considered. We then can normalize the plot from $0-100 \%$ achievable crystallinity, though this makes the implicit assumption that the amorphous and crystalline domains have the same density. From the normalized curves generated in this manner, we may start to collect kinetic data. The Avrami theory for crystallizable materials was previously described in Section 2.4.1. In order to apply the theory, we begin with Equation [2.12]:

$$
V(t)=1-\exp \left(-k t^{n}\right)
$$

This describes the shape of Figure 4.4. We can rewrite this expression in a more usable form for crystallization analysis by taking the double logarithm of both sides and rearranging the terms:

$$
\ln (-\ln [1-V])=n \ln (t)+\ln (k)
$$

We should also note at this point that Figure 4.4 presents a non-linear curve which indicates that several mechanisms are at play over the crystallization period, and that Equation 4.1 is double logarithmic. These factors can cause significant variations in calculated values depending upon 
where the data lie on the transformation curve. For this reason, the work of Lorenzo et al. was consulted and the period between 5-20\% relative crystallization was considered. Their suggestion is that the underpinning theory behind the equation is most accurate in this region where only primary crystallization occurs without impingement of crystals upon each other or the termination of crystal growth ${ }^{94}$. Plotting Equation 4.1 for the above sample over this region gives a highly linear plot as seen in Figure 4.5:

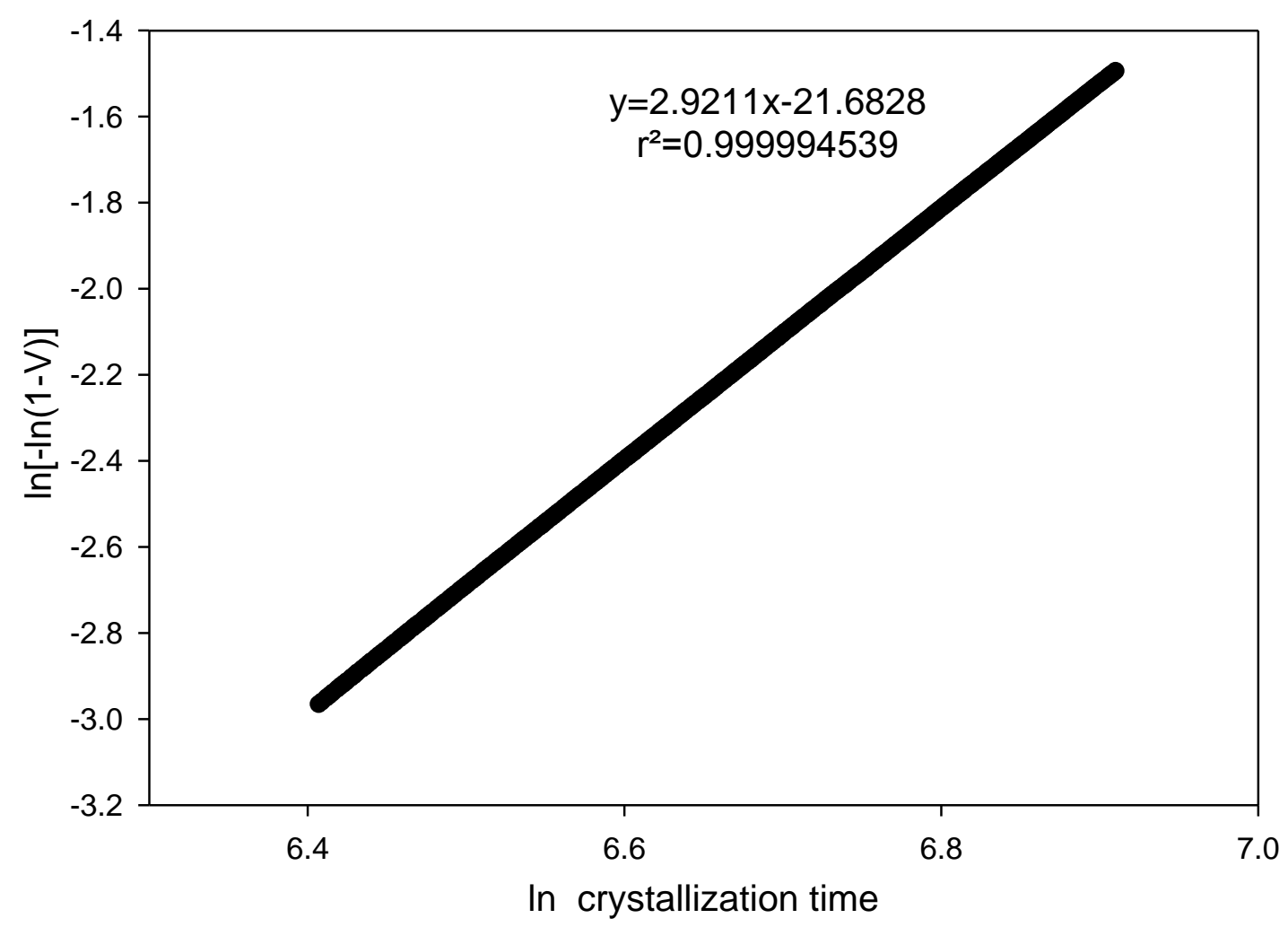

Figure 4.5: Avrami plot of previous sample over 5-20\% conversion range.

The very high $\mathrm{r}^{2}$ value confirms that this is an accurate portion of the curve to select. For both the unfilled and graphene-containing compositions, 3 replicates were analyzed at 90-100-110$120^{\circ} \mathrm{C}$ to generate the Avrami parameters. These are plotted in Figures 4.6 and 4.7: 


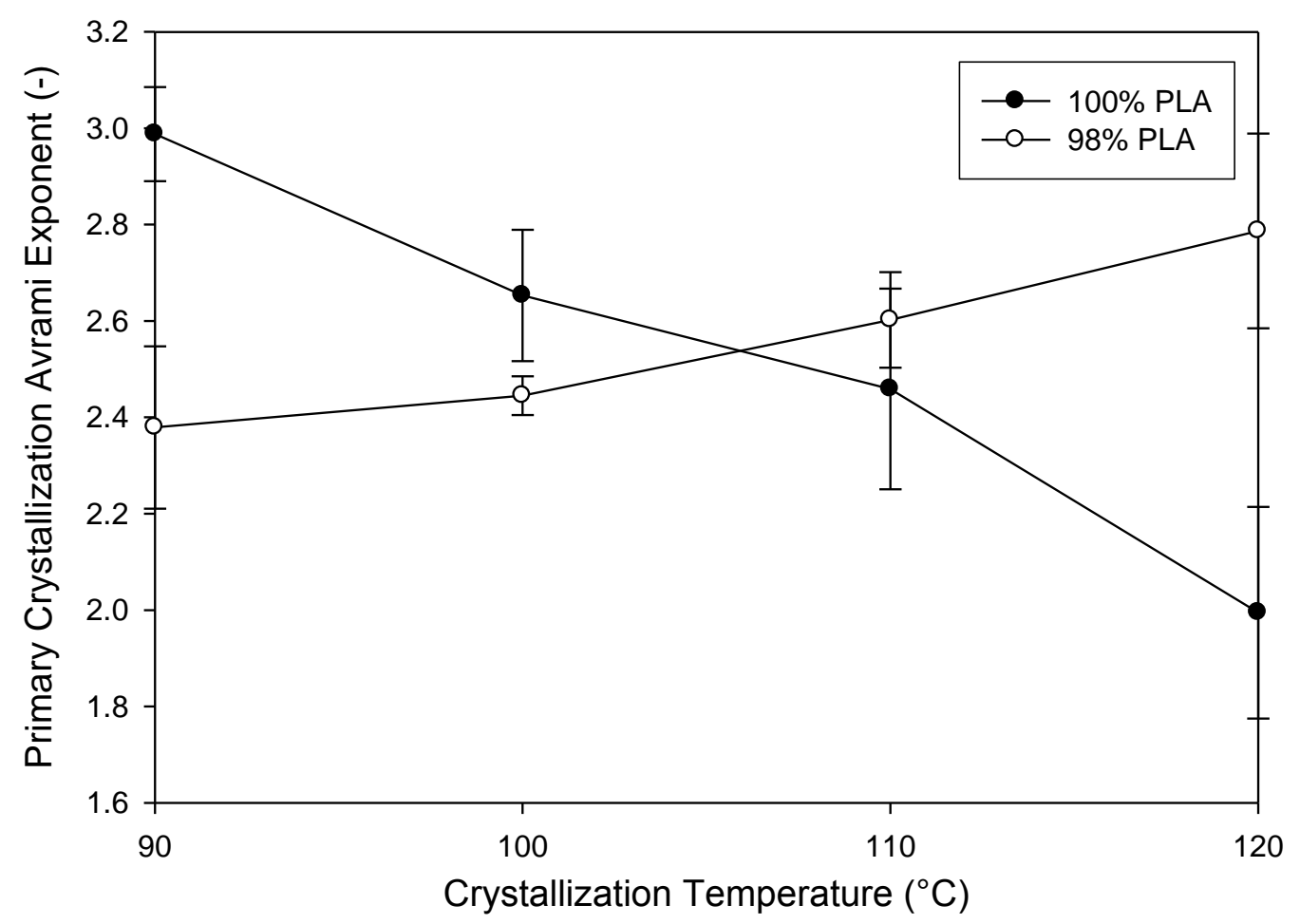

Figure 4.6: Avrami exponent for primary crystallization of $100 \mathrm{~A}$ and $100 \mathrm{G}$ blends.

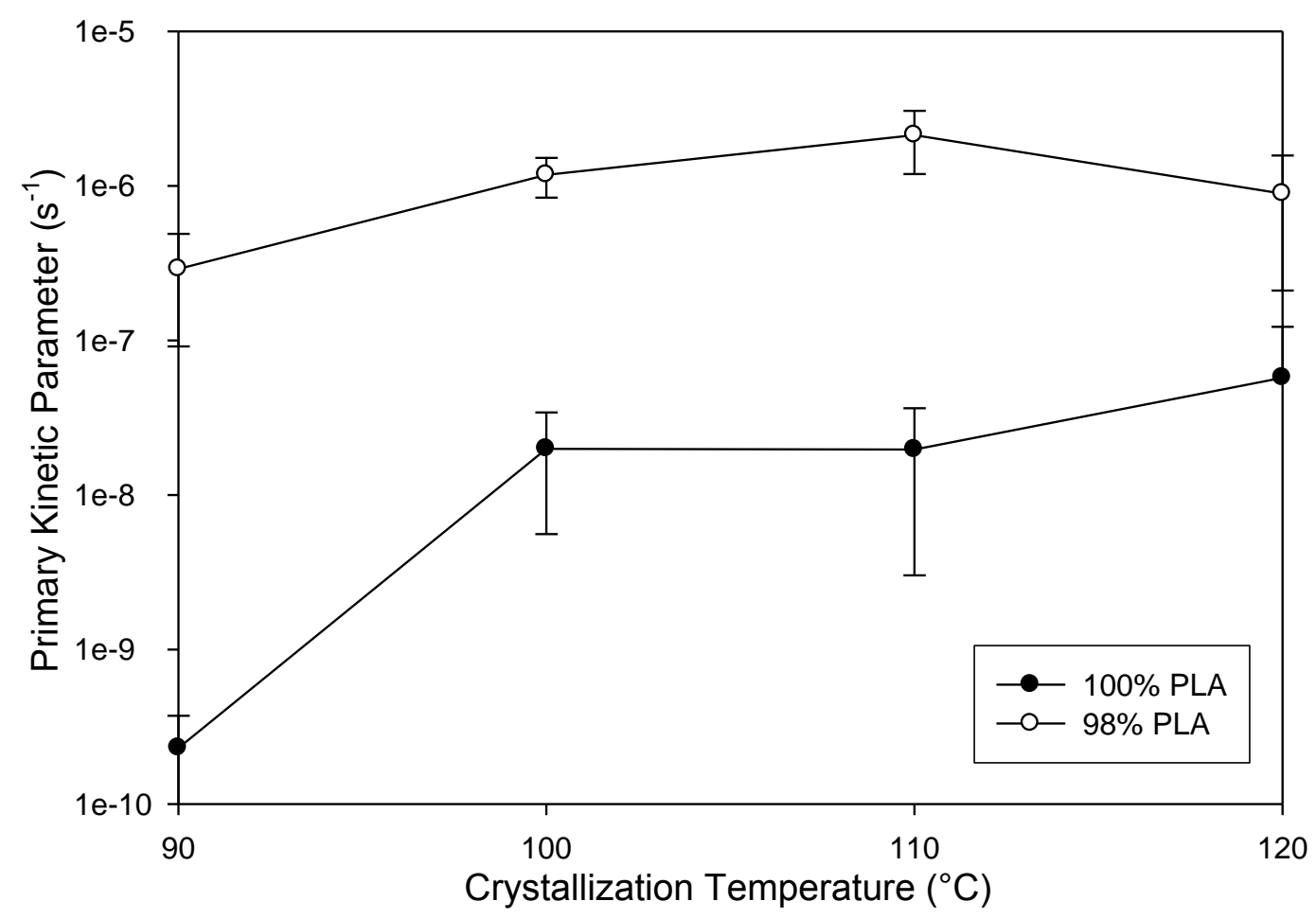

Figure 4.7: Kinetic parameter for primary crystallization of $100 \mathrm{~A}$ and $100 \mathrm{G}$ blends. 
We note that lines are added not to suggest trends, but to connect data points within a set for clarity. A comparison can be made with the data presented in Figure 2.17, the works of Pantani et al. with a variety of processed PLA materials. They determined the Avrami exponent for their PLA samples to be 2.7-2.9 depending on which processing was performed; here within $90-110^{\circ} \mathrm{C}$ it is about 3 , and closer to 2 at $120^{\circ} \mathrm{C}$ for the unfilled sample. The sample that contains graphene has a marginally lower exponent of 2.4 at $90^{\circ} \mathrm{C}$ which increases to about 3 at $120^{\circ} \mathrm{C}$. It should be noted that the extrusion processing was much less severe in their work, only reaching $200^{\circ} \mathrm{C} / 40 \mathrm{rpm}$ as compared to $240^{\circ} \mathrm{C} / 110 \mathrm{rpm}$ in the current research; this different thermal history could cause a change in material properties which would affect the crystallization. However, comparing this data to theirs shows a degree of internal consistency; they measured or modeled maximum PLA crystallinity also using PLA2002D to be about $25 \%$ whereas this value in nearly all cases is around $30 \%$ in the current work $^{85}$.

Since the samples showed exponents ranging from about 2-3 from $90-120^{\circ} \mathrm{C}$, this suggests dimensionality of crystallinity was between plate-like and polyhedral, with spontaneous nucleation. In terms of the kinetic parameter, Pantani et al. measured considerably higher values than this work. Their calculation was based on taking the inverse of the calculated crystallization half-time whereas here it was determined by the intercept of the data presented in Figure 4.5. Therefore, examining their data again in Figure 2.17, the values of $k$ for the extruded material crystallized from the melt (open squares) over the $90-120^{\circ} \mathrm{C}$ range are approximately $10^{-4}$ seconds $^{-1}$. Inverting this we obtain a half-time on the order of $10^{4}$ seconds, which can be compared to the fastest $\left(\sim 130\right.$ seconds for graphene-filled at $\left.110^{\circ} \mathrm{C}\right)$ to the slowest $(\sim 4000-5000$ seconds for unfilled at $120^{\circ} \mathrm{C}$ ) volume-transformation half-times in this work. Due to the inherent sensitivity of the data range selected for analysis, it is unsurprising to find some discrepancies in this area; this is especially true considering they selected the half time at $50 \%$ crystallization compared to tabulating it from data using 5-20\% crystallinity for this work. Furthermore, the slightly modified Avrami equation from their work (which includes a $-\ln (2)$ factor) may also add to this difference.

What is quite clear from Figure 4.7 is there is a strong nucleating and crystal growth-accelerating effect that comes from the addition of $2 \%$ graphene by weight into the PLA phase. The increase in kinetics in this figure is about 1-3 orders of magnitude. This is easily observable from a 
simple dynamic DSC test on the PLA/graphene blend, which exhibited crystallization during cooling from the melt at $10^{\circ} \mathrm{C} /$ minute as shown in Figure 4.8:

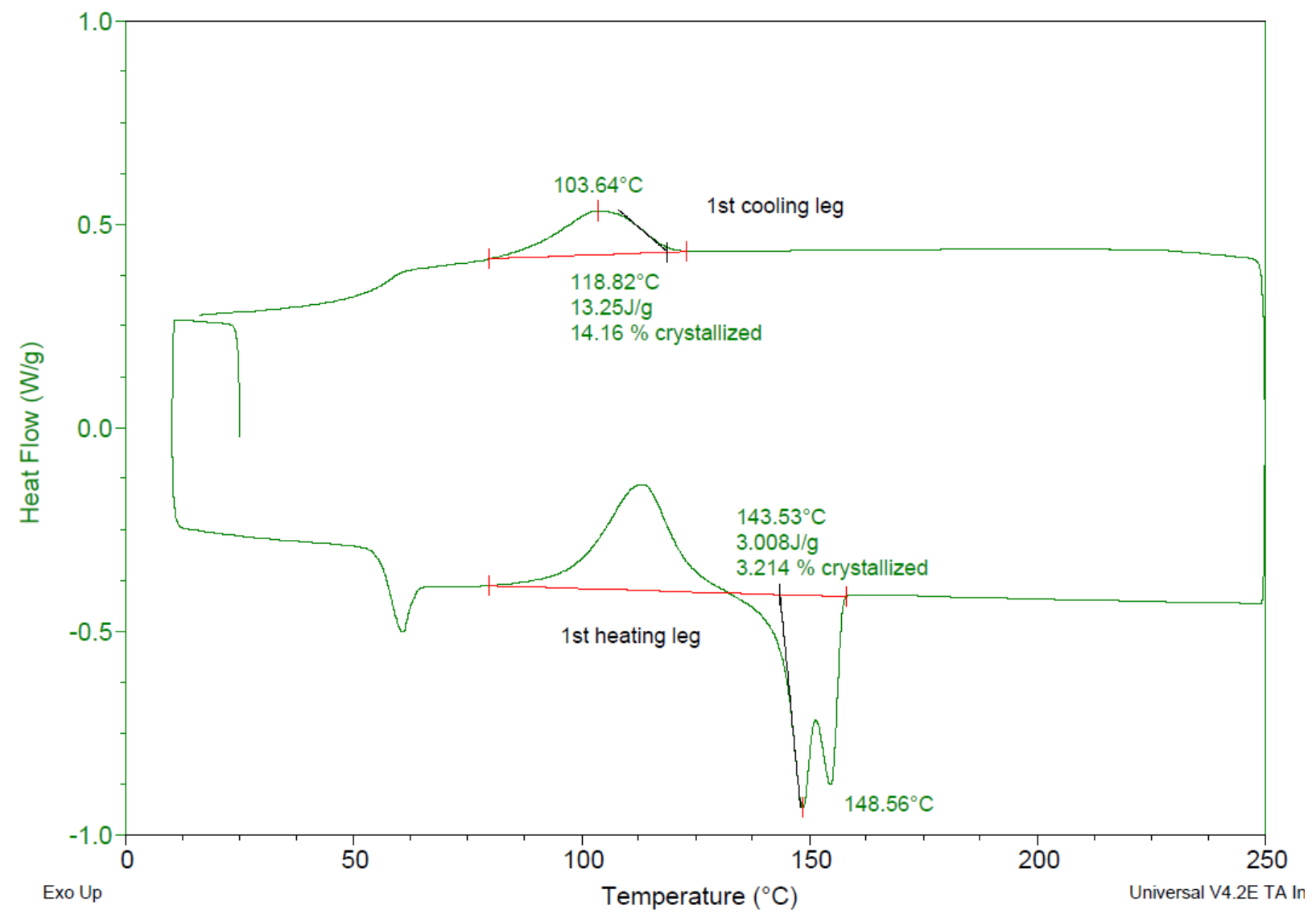

Figure 4.8: Crystallization on cooling for sample containing 98/2 PLA/graphene.

This effect is not present for unfilled PLA, nor any other sample. Next, if the ratio of the time for $75 \%$ conversion to $25 \%$ conversion as suggested by Avrami in Table 2.2 is taken, the data for these PLA compositions and temperatures is on the order of 1.6-1.9, suggesting plate-like to polyhedral growth ${ }^{81}$. However, since the values for both the unfilled and filled composition are all in this range, it would not seem that the graphene affects the shape of crystal growth, only the kinetics.

These experiments have shown that the crystallization of PLA is describable by the traditional Avrami theory during primary crystallization. While the crystallization is slow, it can be accelerated by orders of magnitude with the inclusion of a nucleating agent such as graphene; this has been previously noted (e.g. ${ }^{95}$ ). However, the graphene does not appear to alter the 
crystal growth shape. The next step is to determine the effect of crystallinity and other treatments on chemical stability.

\subsubsection{Effect of Treatment on Initial Crystallinity of PLA and PLA/Graphene Composite.}

In spite of collecting the crystallization kinetic data, heat treatment was not performed according to an Avrami-style protocol. This is because of inherent error in calculating those kinetics and that initial experiments showed that molded parts (especially the large flexural bars) tended to warp if heat-treated for significant times above $90^{\circ} \mathrm{C}$. Therefore, any annealed pellets, panels or flexural bars were all treated in the same manner to eliminate variation in exposure time. Heat treatment was carried out at $80^{\circ} \mathrm{C}$ for 48 hours in an oven without convection. The pellets were arranged in aluminum trays in roughly 30 gram batches, while molded parts were sandwiched between metal trays that were in turn covered with aluminum foil to prevent warping. After removal from the oven, the samples were placed on a large metal table at room temperature to quickly remove heat from the material to halt any further thermal effects.

After cooling, a few milligrams of the pellets or small pieces snipped from the molded parts were analyzed in the DSC for dynamic analysis. The initial crystallinities of the materials in both pellet and molded part form are given in Figure 4.9: 


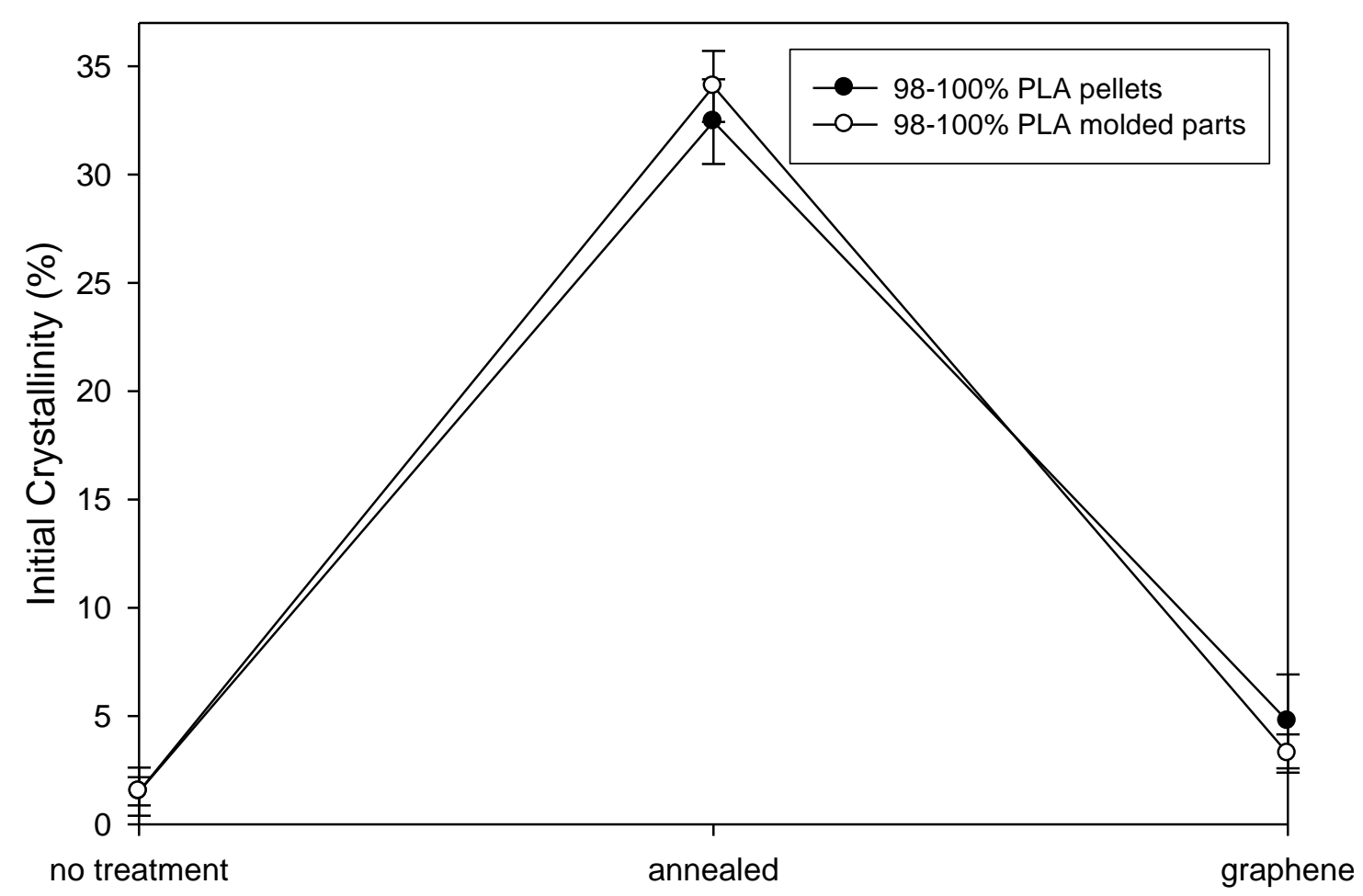

Figure 4.9: Initial crystallinity of 98-100\% PLA parts and pellets by treatment option.

Here it is clear that annealing the materials is the best way to achieve appreciable crystallinity within either the pellets or molded pieces. It is also internally consistent with previous results where the maximum PLA crystallinity achieved is on the order of 30-35\%. We also note that in spite of the ability of the graphene to accelerate crystallization from the melt, there is not much crystallinity formed due to the overall slow crystallization rate. Both the untreated and graphene-containing PLA samples are for all intents and purposes totally amorphous while the annealed samples are at maximum crystallinity; this should provide conclusive evidence as to the crystallinity effect on the stability of PLA in the face of solvent or hydrolytic degradation.

\subsubsection{Solvent Degradation of PLA and PLA/Graphene Composites.}

Degradation in various media was carried out to understand the stability of the materials that would come in contact with real-world situations that may be acidic or basic. Compression molded parts in the form of $30 \mathrm{~mm} * 30 \mathrm{~mm} * 3 \mathrm{~mm}$ squares were measured for weight, crystallinity, and viscosity changes as a result of exposure at $50^{\circ} \mathrm{C}$ over a period of 42 days. The 
effect of solvent degradation on the weight change of $98-100 \%$ PLA materials is shown in Figures 4.10-12:

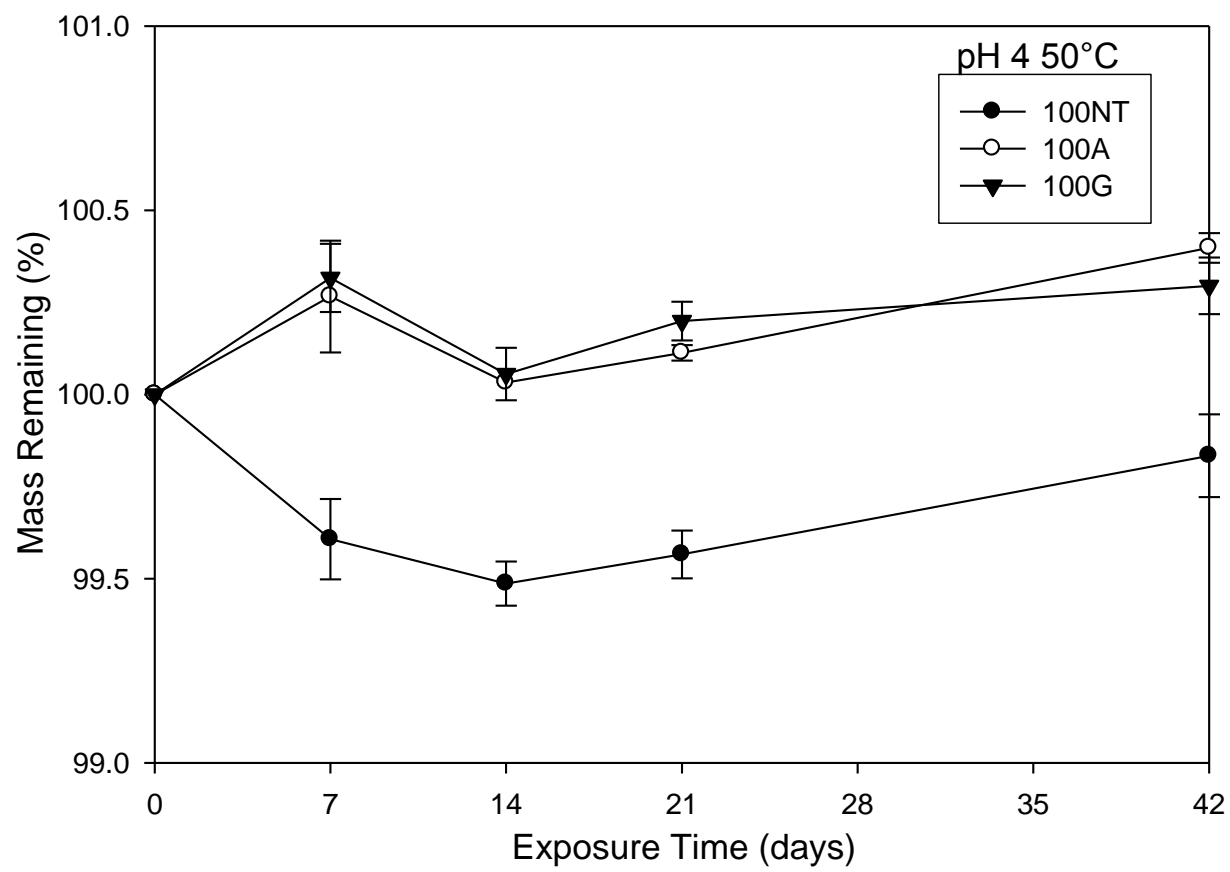

Figure 4.10: Weight loss of PLA and PLA/graphene samples during exposure to acetic acid buffer.

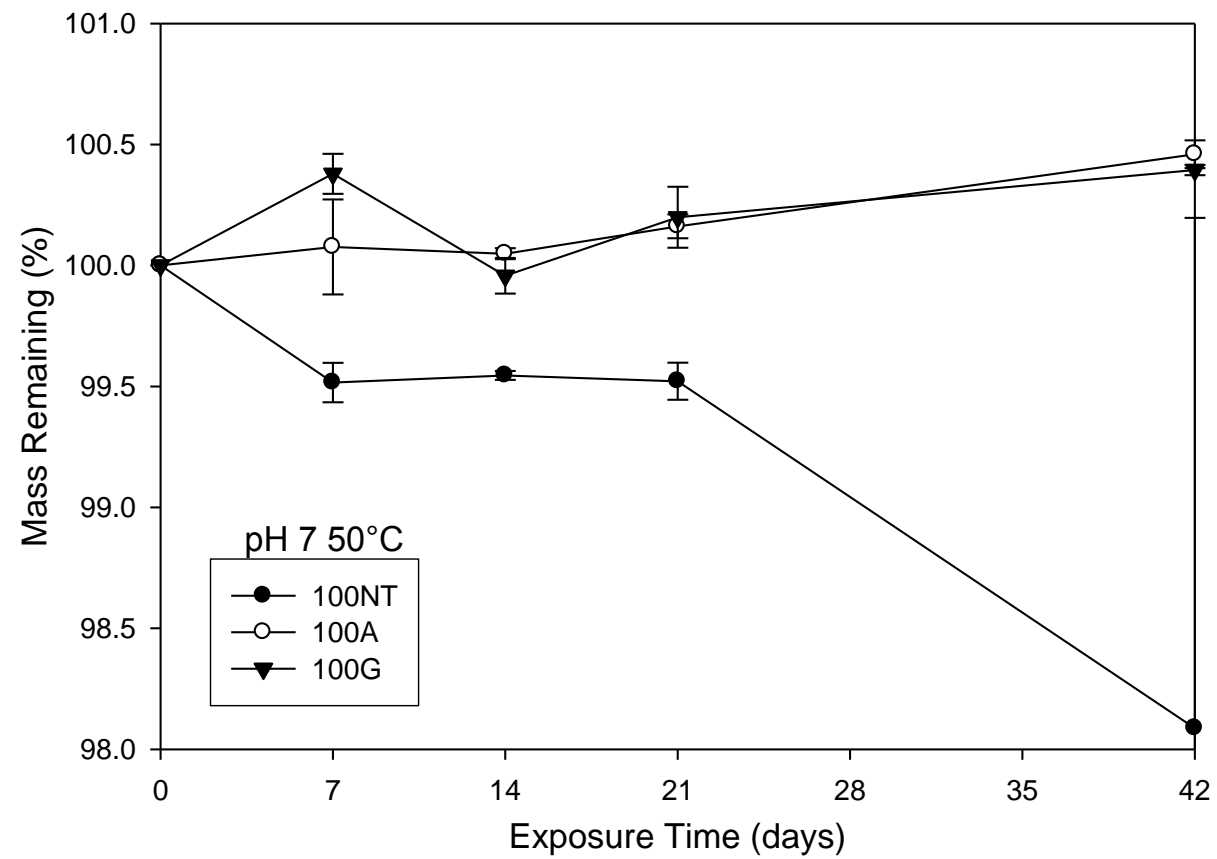

Figure 4.11: Weight loss of PLA and PLA/graphene samples during exposure to distilled water. 


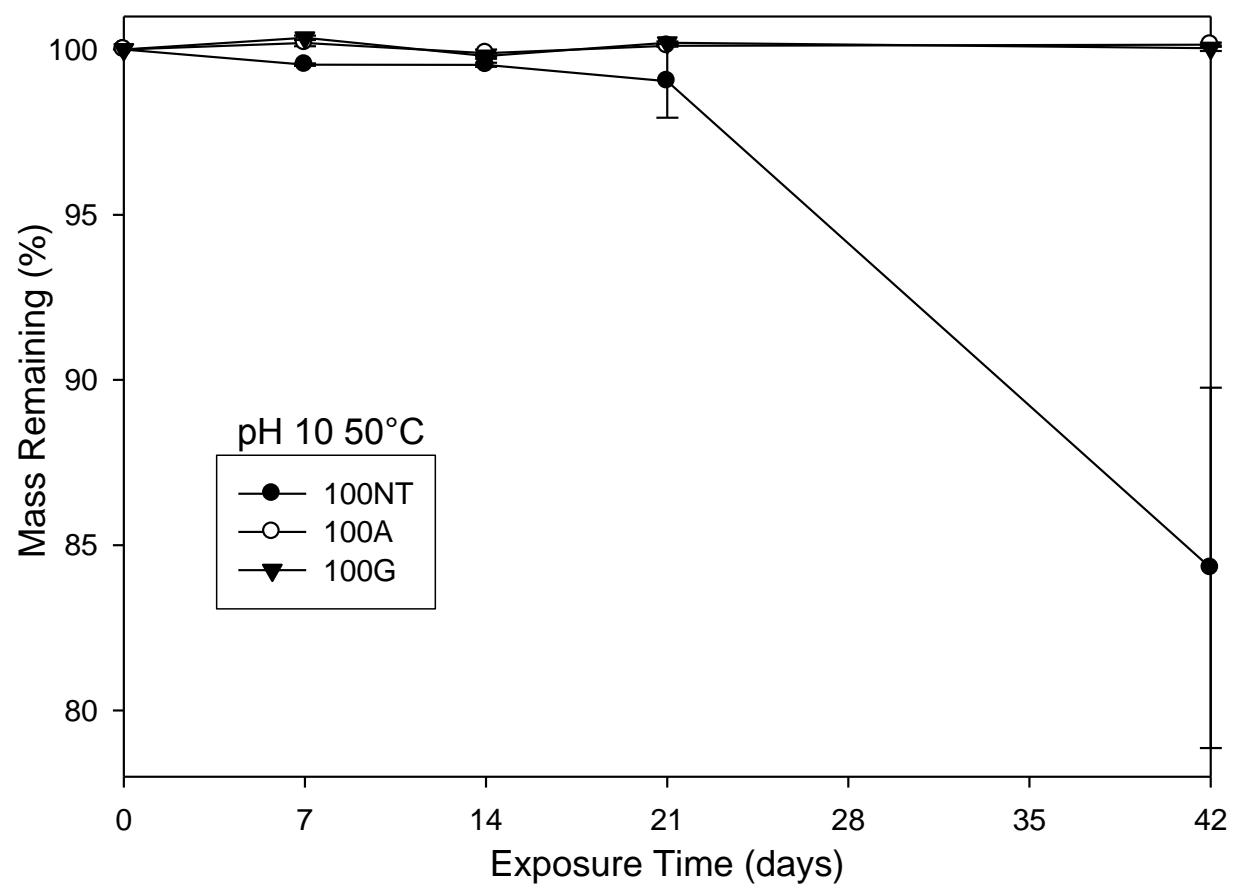

Figure 4.12: Weight loss of PLA and PLA/graphene samples during exposure to carbonate buffer.

In both the acetic acid buffer and distilled water solution, the weight change over 6 weeks degradation is essentially neglible. Recalling Figure 2.2, it was observed that PLA was most stable around $\mathrm{pH} 4$ and the rate of degradation increased as the $\mathrm{pH}$ moved further in either direction. It is also clear here that the basic solution was highly aggressive to the untreated PLA, causing around 15\% mass loss after 42 days exposure. This too was suggested in Figure 2.2, that hydroxyl attack which cleaves off a lactide molecule is a likely mechanism for the degradation of PLA polymer. Since mass was lost only during long duration attack by base, it also seems likely that bulk erosion is the more prominent mechanism of degradation, where species diffuse into the material and attack the polymer chains. Then, after significant damage is done to allow pores or channels to open through the bulk of the sample, the degraded portions can diffuse out and finally the material begins to fall apart. This also suggests that incorporating barriers to diffusion either by imparting crystalline domains or adding the graphene platelets should retard the bulk degradation process. However, the untreated sample shows that after diffusional resistance is overcome, the PLA will begin to break down. 
The untreated compression molded samples were also analyzed by DSC to determine the effects of initial crystallinity and if the crystalline properties changed with exposure to the liquid media. The crystallinity changes for the PLA materials in each solvent are presented in Figures 4.13-15:

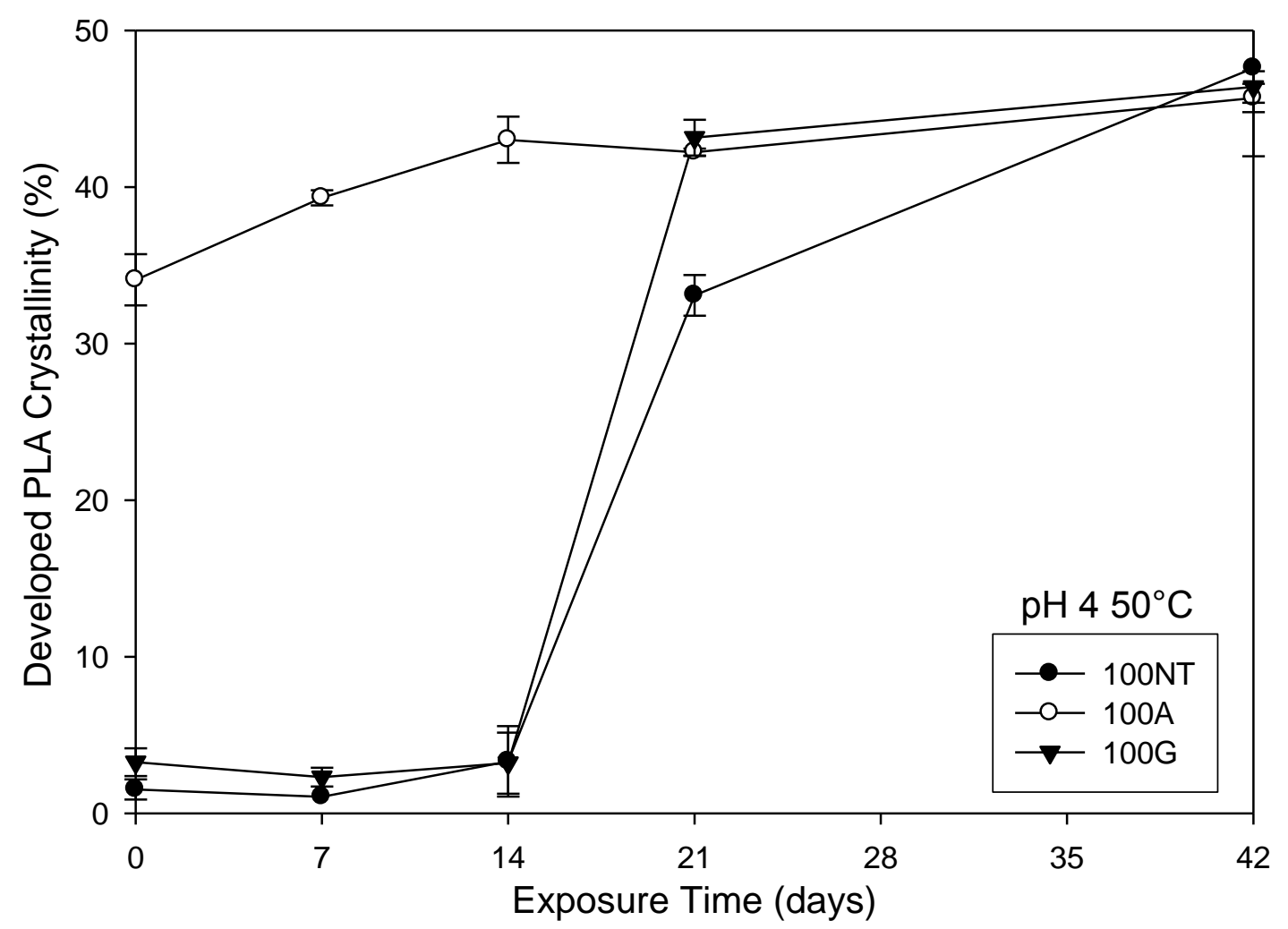

Figure 4.13: Development of crystallinity in PLA and PLA/graphene during exposure to acetic acid buffer. 


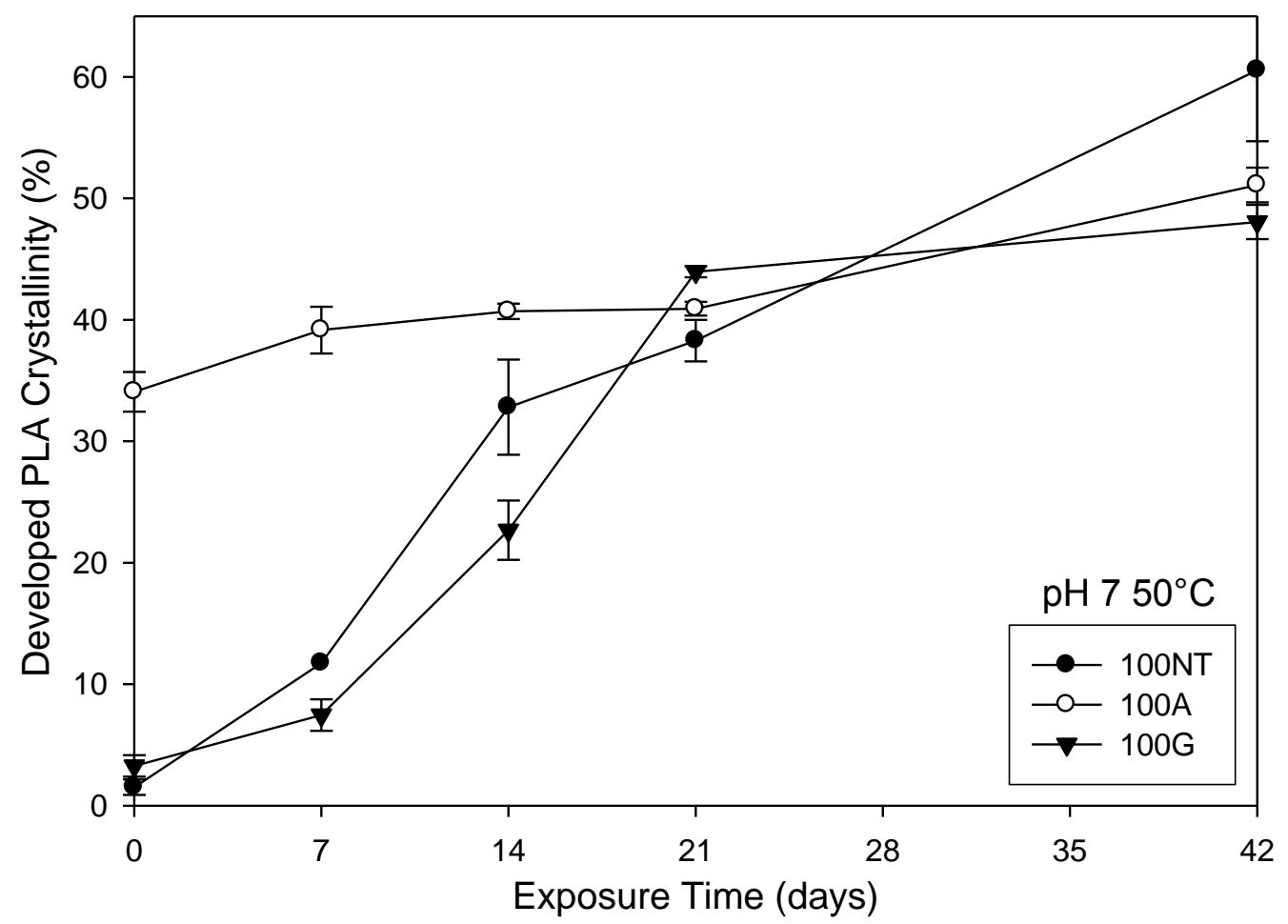

Figure 4.14: Development of crystallinity in PLA and PLA/graphene during exposure to distilled water.

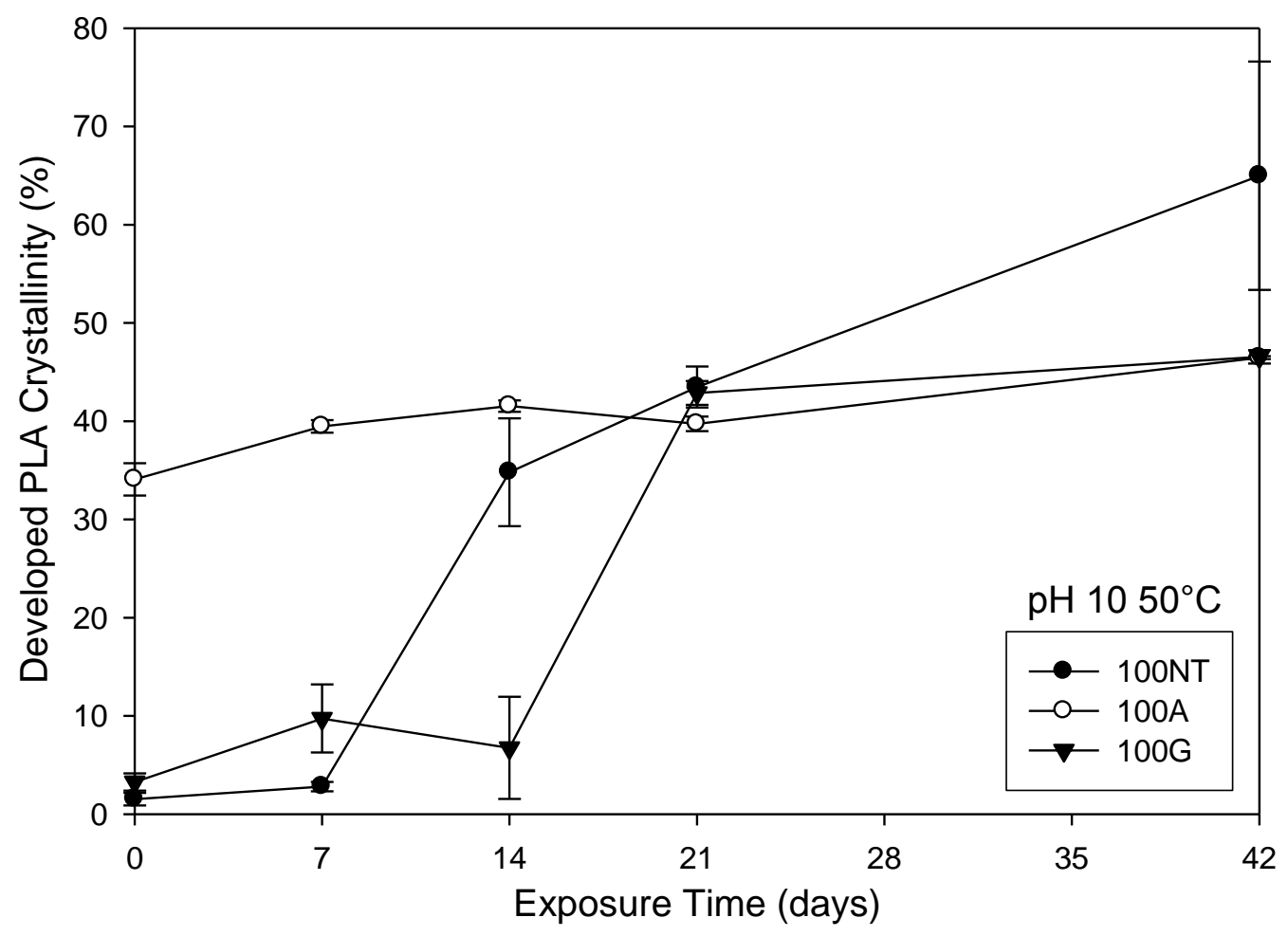

Figure 4.15: Development of crystallinity in PLA and PLA/graphene during exposure to carbonate buffer. 
Earlier, Fu et al. showed an increase in crystallinity as their PLGA fibers were degraded in a phosphate-type buffer ${ }^{37}$. This has been attributed to two processes: amorphous sections of the bulk can be preferentially degraded away and then diffuse out of the material, leaving behind a smaller mass that contains a higher mass of crystalline domains, or a slightly different process sometimes called chemicrystallization can take place. An explanation for this phenomenon is that as the polymer backbone is broken down, the smaller fragments and oligomers that are generated are capable of reforming into smaller crystallites, perhaps due to enhanced mobility which may allow easier formation of hydrogen bonding ${ }^{96}$.

For the untreated and graphene-filled samples, a monotonic rise in crystallinity was observed as expected. The annealed samples experienced a slight increase in crystallinity. The development of final crystallinity increased as the media became more basic, again confirming that PLA is most susceptible to attacking hydroxyl ion. Further, because neither the annealed nor the graphene sample lost any appreciable amount of weight, we can likely say this was a result of species diffusing into the bulk, scissioning the polymer backbone and causing chemicrystallization to take place. However, since there was mass lost for the untreated sample, we cannot say for certain whether chemicrystallization or the preferential surface degradation of amorphous regions lead to the increase in crystallinity; likely it is some combination of both.

Another method used to characterize the extent of solvent degradation was by the change in viscosity. These samples were tested with the parallel-plate rheometer at $170^{\circ} \mathrm{C}$ in oscillatory mode under $12 \%$ strain. The effects of solvent exposure on the viscosity loss on untreated PLA samples is shown in Figures 4.16-18: 


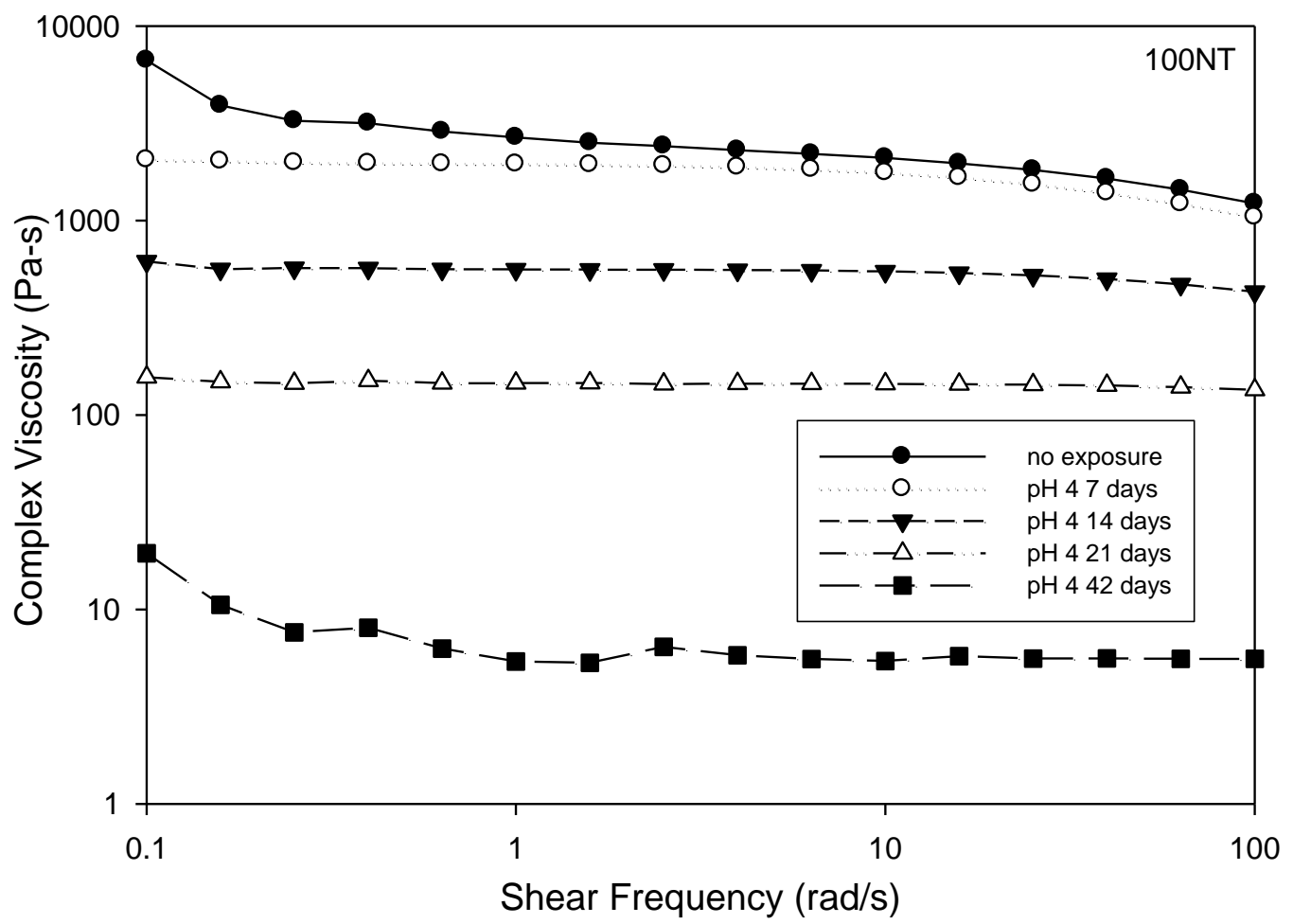

Figure 4.16: Viscosity change of untreated PLA after exposure to acetic acid buffer.

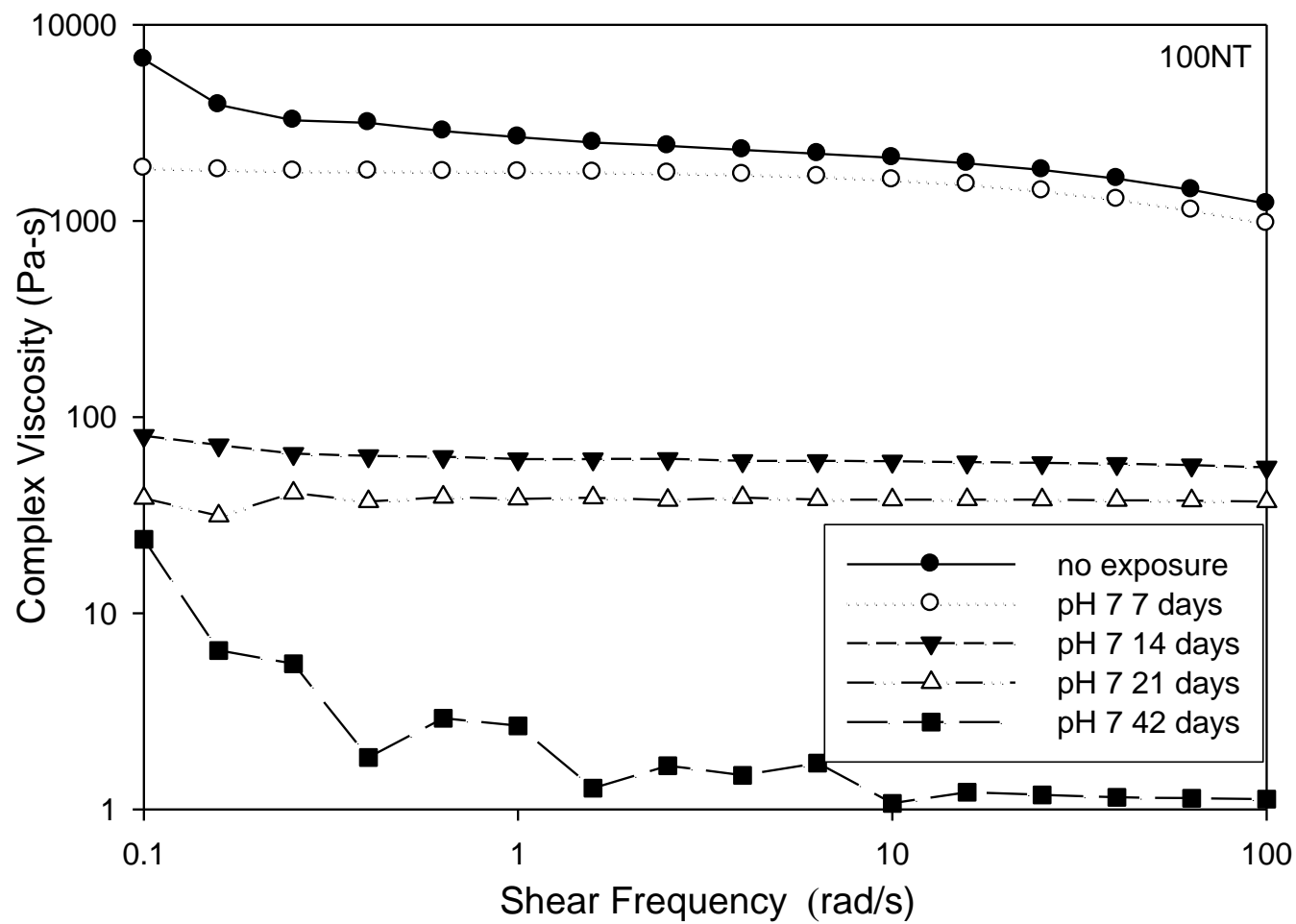

Figure 4.17: Viscosity change of untreated PLA after exposure to distilled water. 


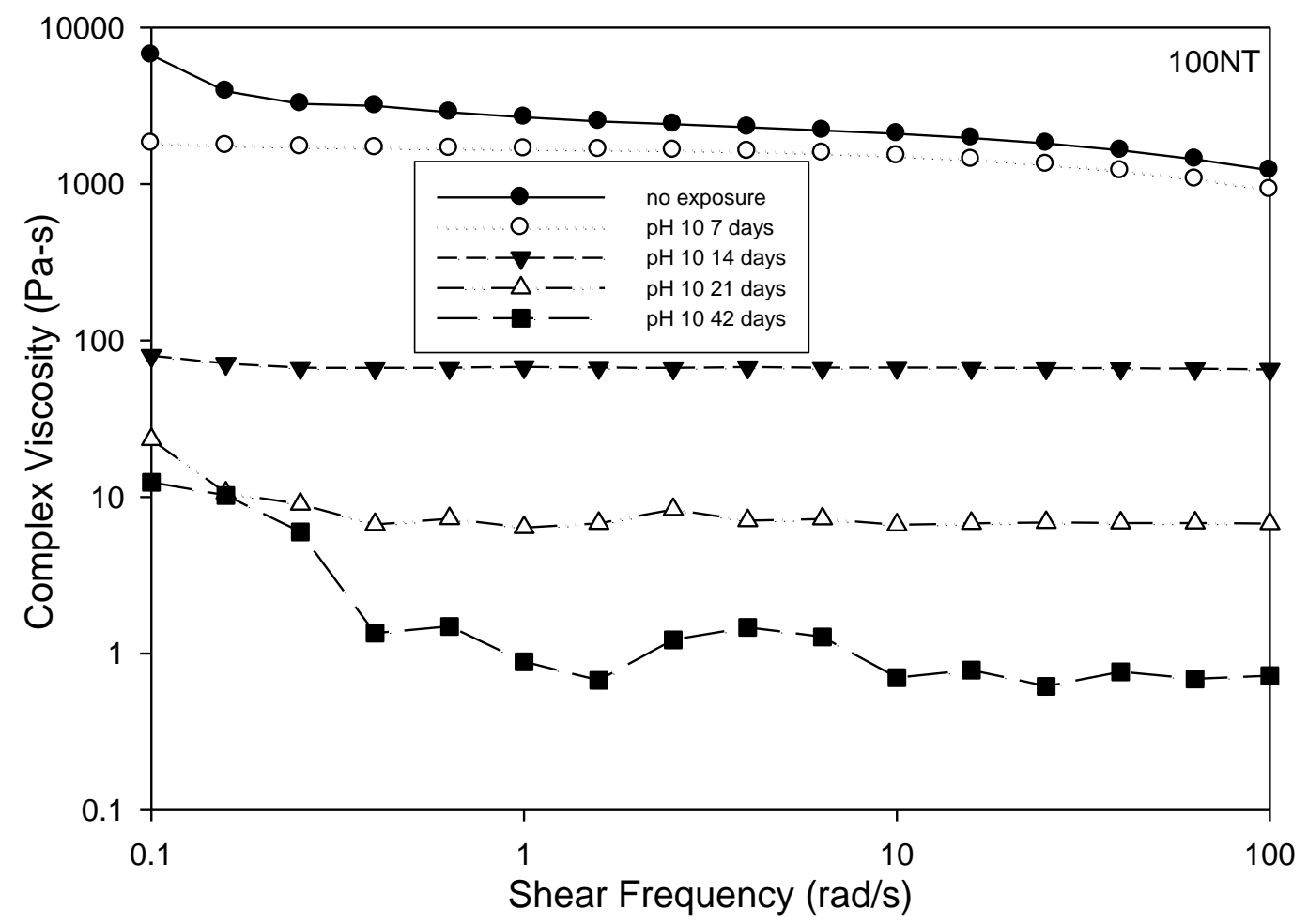

Figure 4.18: Viscosity change of untreated PLA after exposure to carbonate buffer.

From Figures 4.16-18, it is clear that significant viscosity loss has occurred by about 14 days exposure in all of the media, and after 42 days exposure the material has very little viscosity remaining. Samples degraded in the acidic solution have slightly higher viscosities during degradation, which again shows that PLA is more stable around the $\mathrm{pH} 4$ mark as shown in Figure 2.2. However, after 42 days of exposure (a comparable situation to 1 year outdoor aging $^{63}$ ) these materials have negligible viscosity regardless of the attacking solution. In any case since the PLA-only blends undergo tremendous degradation in all media therefore we will show the results of degradation in distilled water hereon. To confirm this is true, now the annealed and graphene viscosity loss curves are also shown as Figures 4.19-20 for distilled water degradation: 


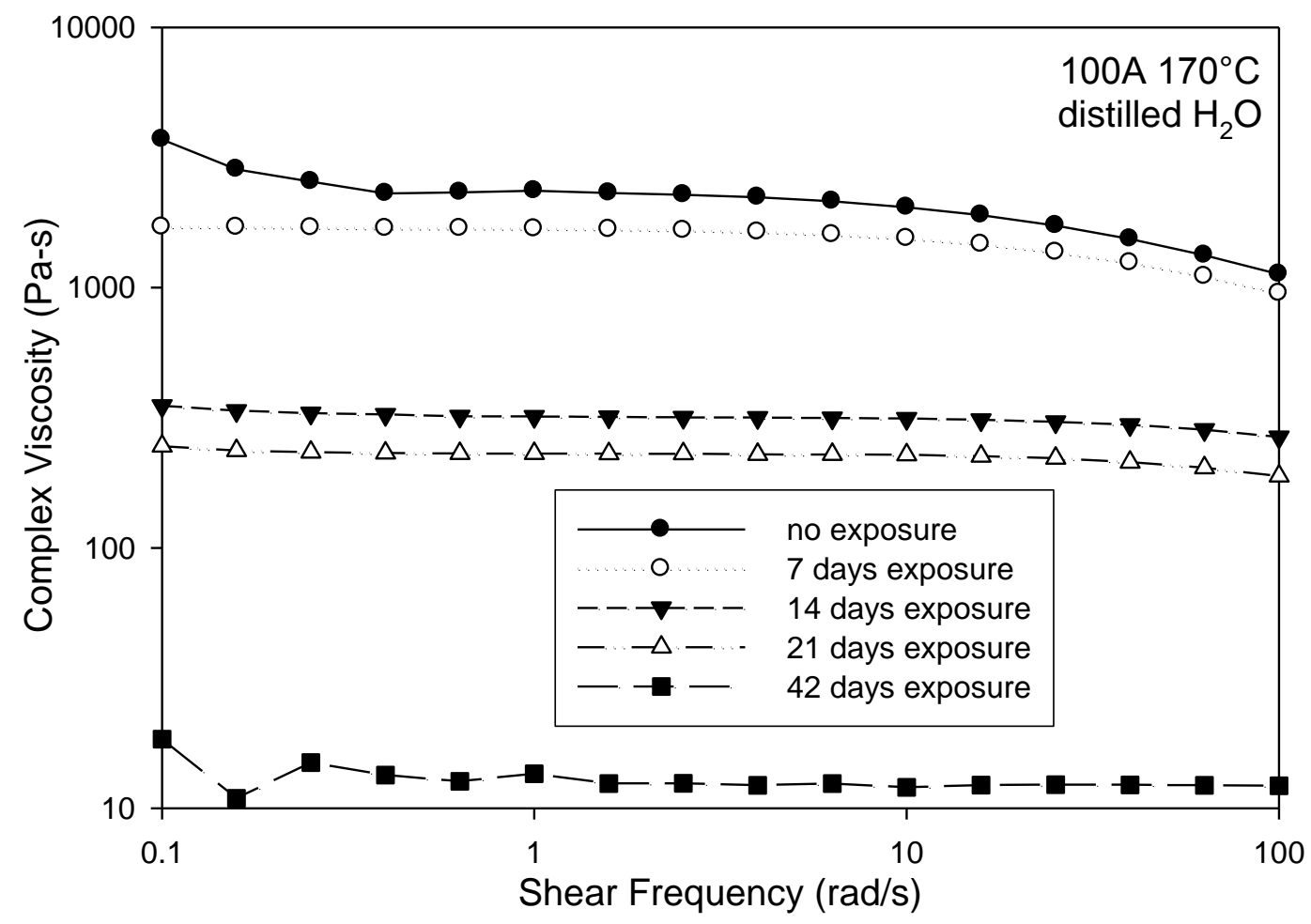

Figure 4.19: Viscosity change of annealed PLA after exposure to distilled water.

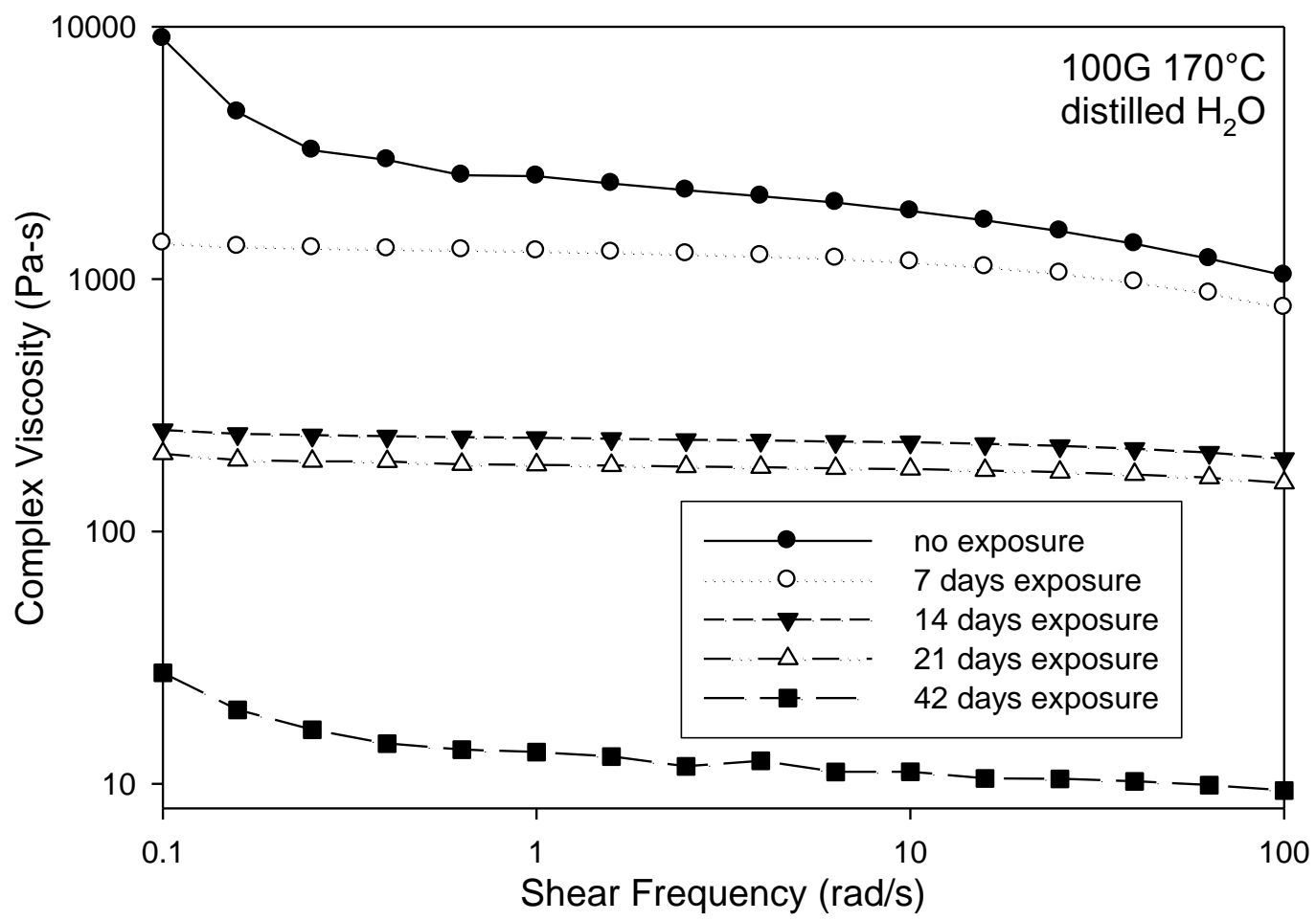

Figure 4.20: Viscosity change of PLA/graphene after exposure to distilled water. 
Like the previous samples, there is significant viscosity loss by 14 days exposure in all cases. However, by the end of degradation there is slightly more residual viscosity in both the annealed and graphene-containing samples but this effect is mostly insignificant. Another way to plot a comparison between the materials is to choose a shear frequency and show the effect of exposure time and treatments. Figure 4.21 is shown for distilled water exposure:

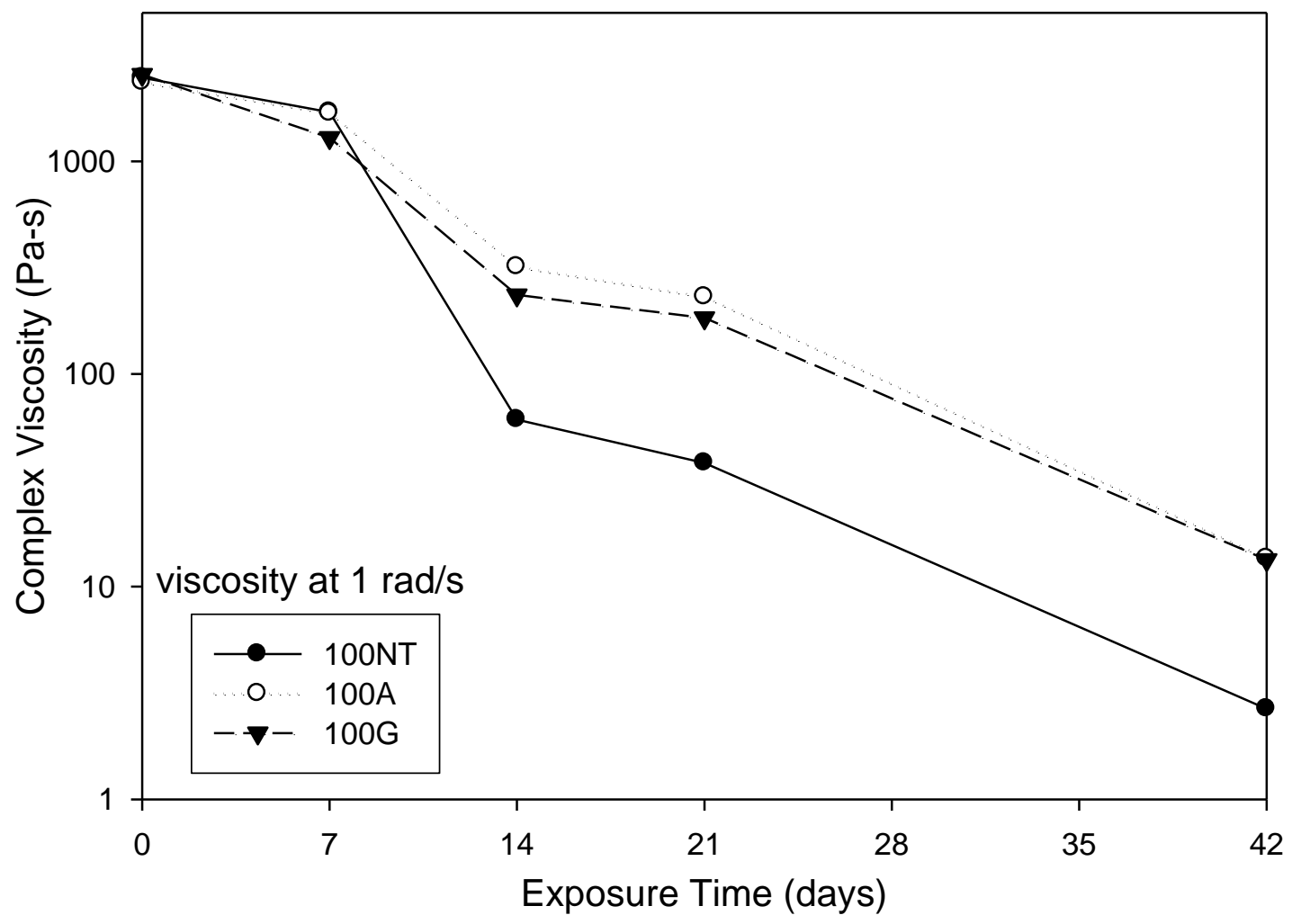

Figure 4.21: Comparison of viscosity loss between varying PLA treatments.

Here again we can see that all the samples undergo tremendous loss of viscosity (and hence molecular weight) over the simulated one year exposure. From this graph, we can see that the initial data point for all samples (at $1 \mathrm{rad} / \mathrm{s}$ shear frequency) is about $2500 \mathrm{~Pa}-\mathrm{s}$ whereas at the end of degradation it is roughly $10 \mathrm{~Pa}-\mathrm{s}$, a reduction of over 99\%. Clearly, none of these 98 $100 \%$ PLA materials with any treatment have the required hydrolytic stability for a durable application at elevated temperature and exposure to attacking media. 
In conjunction with solvent degradation, hydrolytic degradation in the presence of humidity was carried out on pellet samples as well as compression molded flexural bars as described in Section 3.3.6. Those results are the next to address for the PLA and PLA/graphene composites.

\subsubsection{Hydrolytic Degradation of PLA and PLA/Graphene Composites.}

\subsubsection{Effect of Hydrolytic Degradation on Mechanical Properties.}

Besides maintaining molecular weight, any material used for a durable application must also maintain its physical integrity during use. Flexural bars created by compression molding were aged in a humidity chamber at $50^{\circ} \mathrm{C}$ and roughly $100 \%$ relative humidity for up to 42 days, or 1 year of equivalent outdoor exposure to test the change in mechanical properties. Ideally, parts would be fabricated by injection molding to keep pieces as consistent and within the tightest tolerances, but as graphene samples could not be produced reliably by extrusion and batches large enough for injection molding were infeasible to make, the parts were compression molded and each piece was checked carefully for its dimensions. To perform the aging, a custom rack was built out of steel mesh plates that were connected by stainless steel bolts and set apart with PVC spacers, shown here in Figure 4.22:

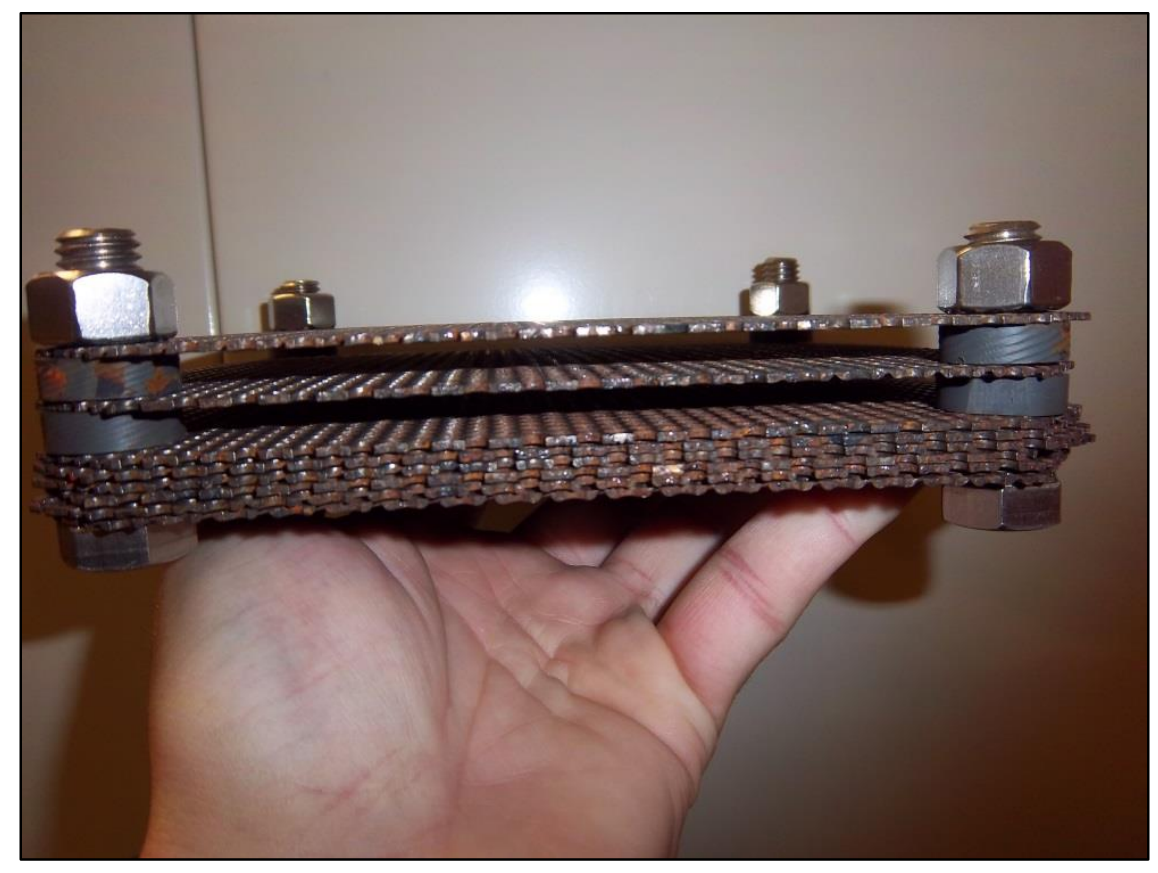

Figure 4.22: Metal rack for hydrolytic aging of flexural bars. 
This was done so as to allow the humidity access to all surfaces of the bars and to ensure that no additional stresses were placed on the parts during aging. These were then aged up to 42 days (in some cases) in the humidity chamber, which under the example given by Harris et al. would be equivalent to about one year outdoor exposure in Florida ${ }^{63}$. The initial flexural modulus, strength and ductility as measured by the Instron 5567 are shown in Figures 4.23-25:

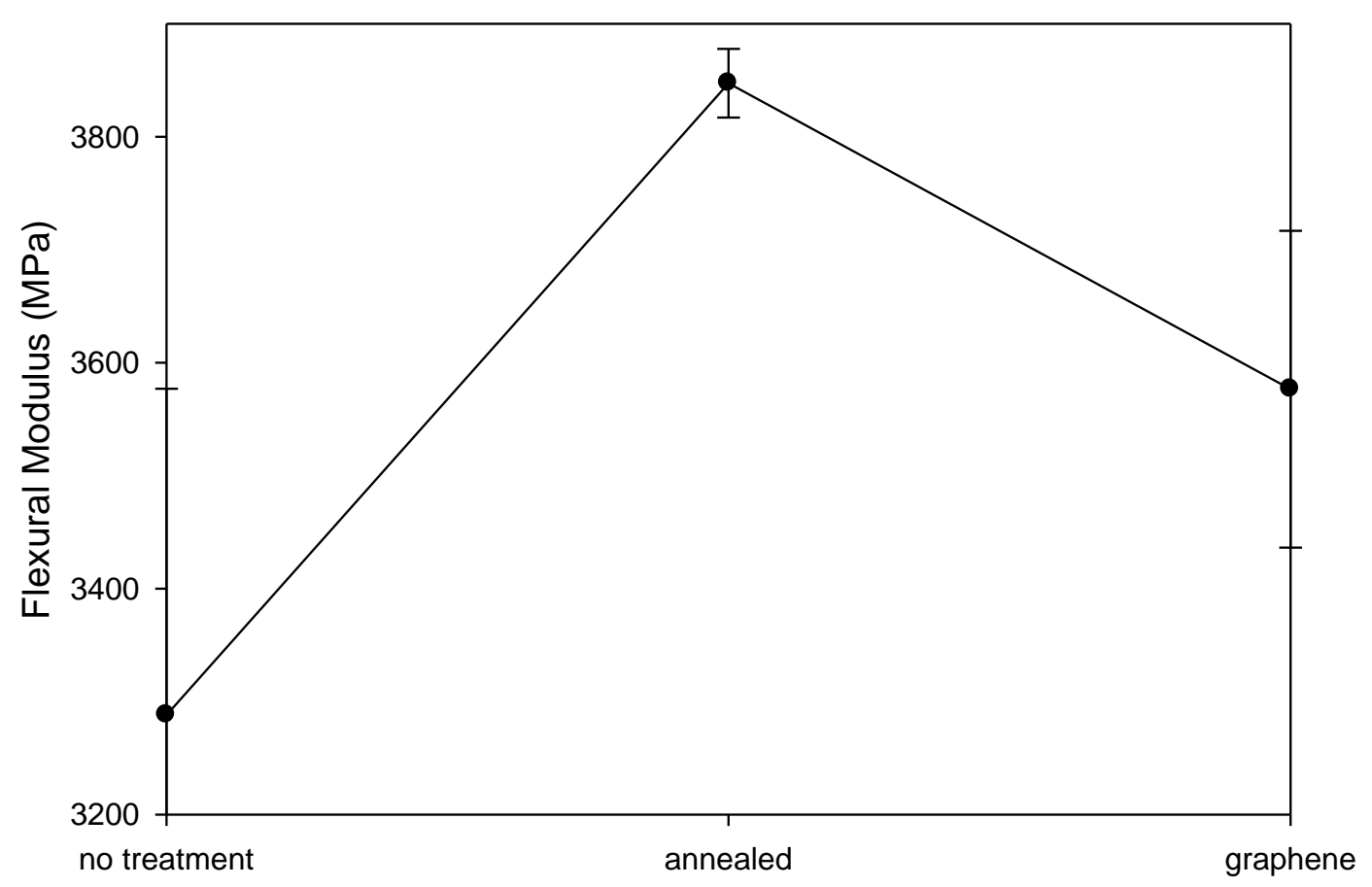

Figure 4.23: Initial flexural modulus of untreated, annealed and PLA/graphene materials. 


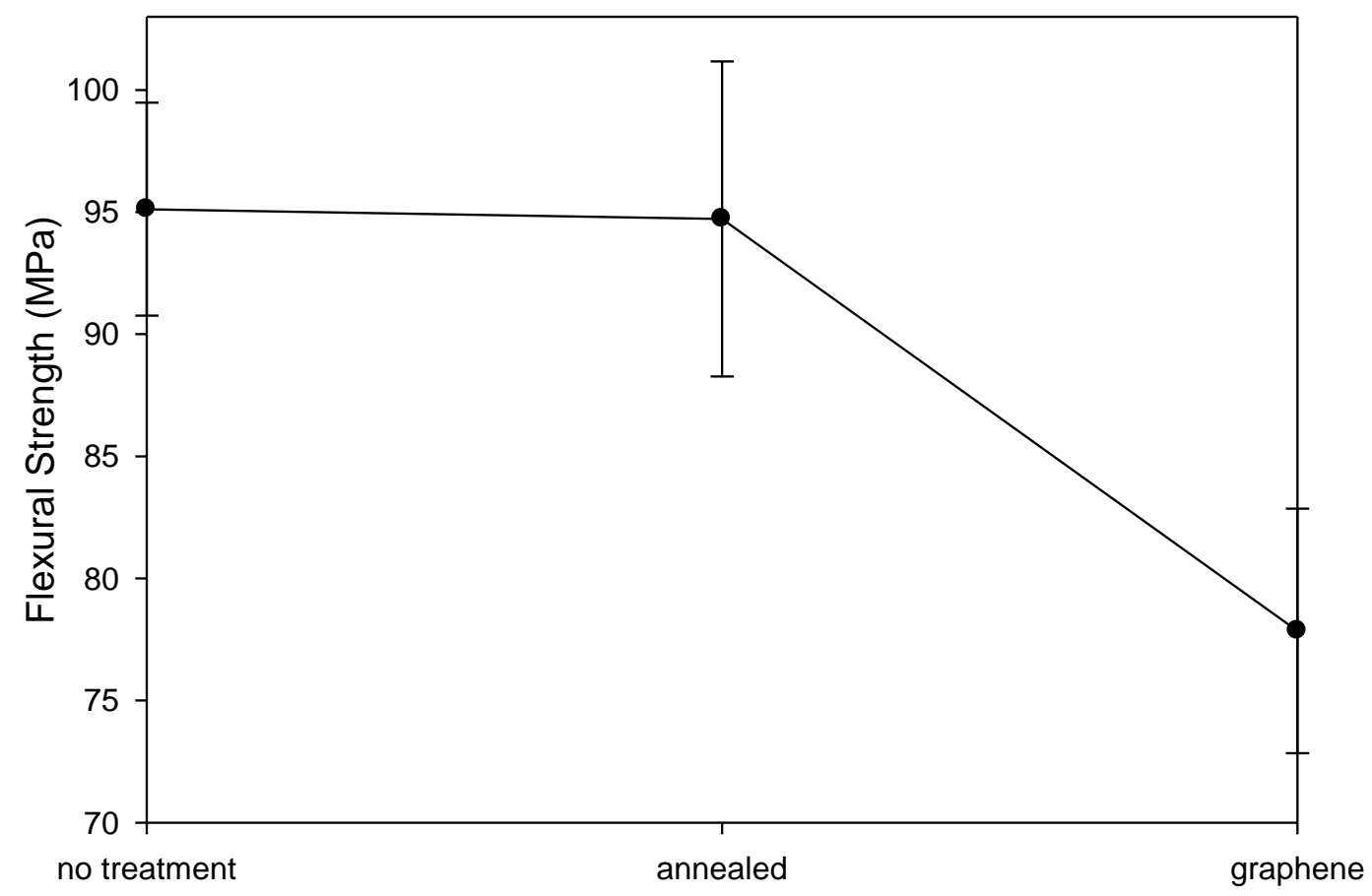

Figure 4.24: Initial flexural strength of untreated, annealed and PLA/graphene materials.

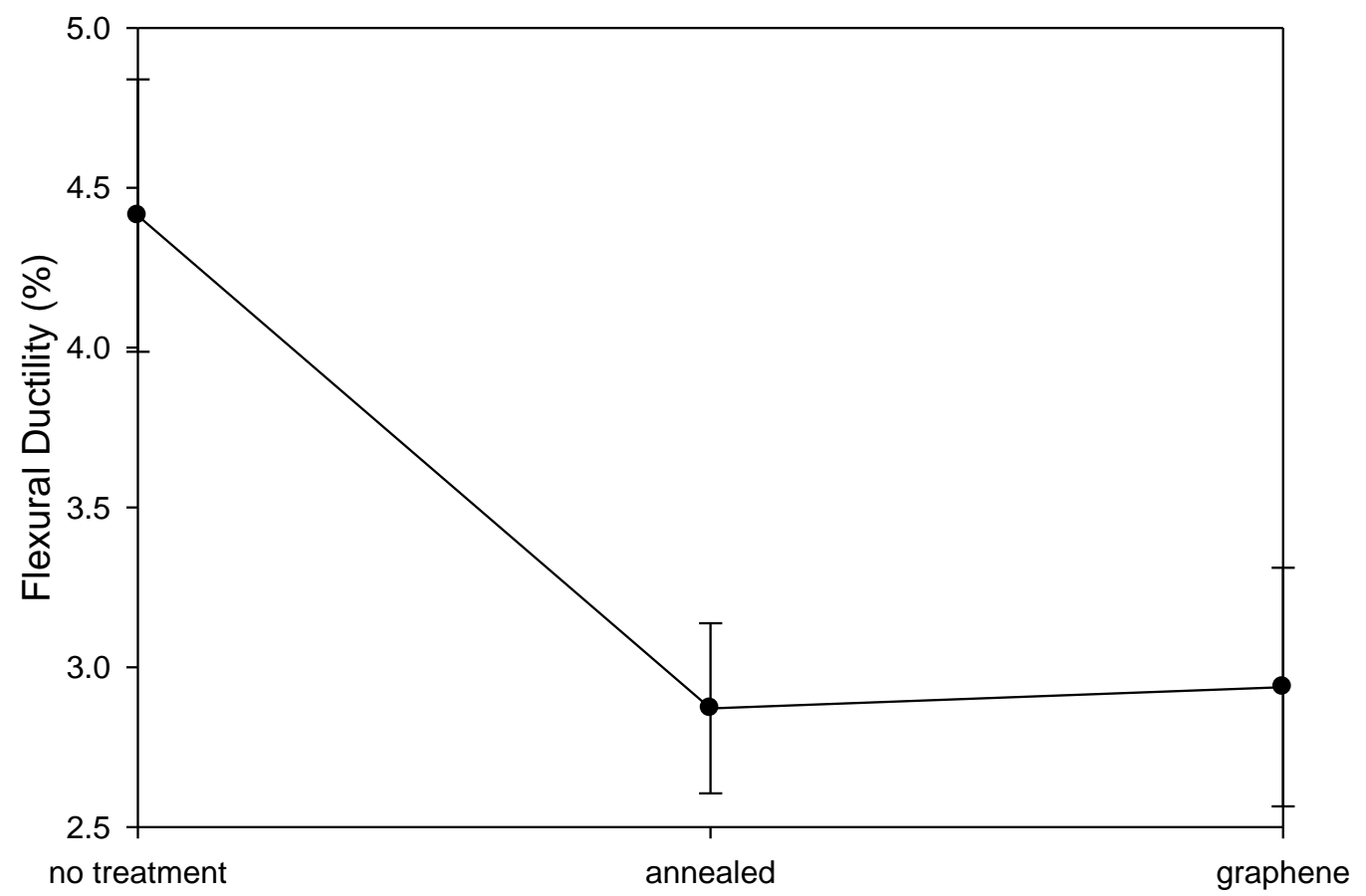

Figure 4.25 Initial flexural ductility of untreated, annealed and PLA/graphene materials. 
Here it is clear that the additional treatments to the PLA polymer impart slight stiffening by increasing the flexural modulus, but this benefit is lessened somewhat as the materials become more brittle. The crystalline domains of the annealed sample are formed by enhanced hydrogen bonding between adjacent polymer chains which make the material less ductile, and the fact that it is difficult to fully disperse nano-fillers into polymer matrices means that aggregates of filler exist which can act as stress concentrators. The data are not dissimilar to those measured by Harris et al., though they used a slightly more crystalline grade of PLA ${ }^{63}$.

As the materials age, we expect the mechanical properties to worsen, and therefore the properties are plotted as a percentage of the initial value in Figures 4.26-28:

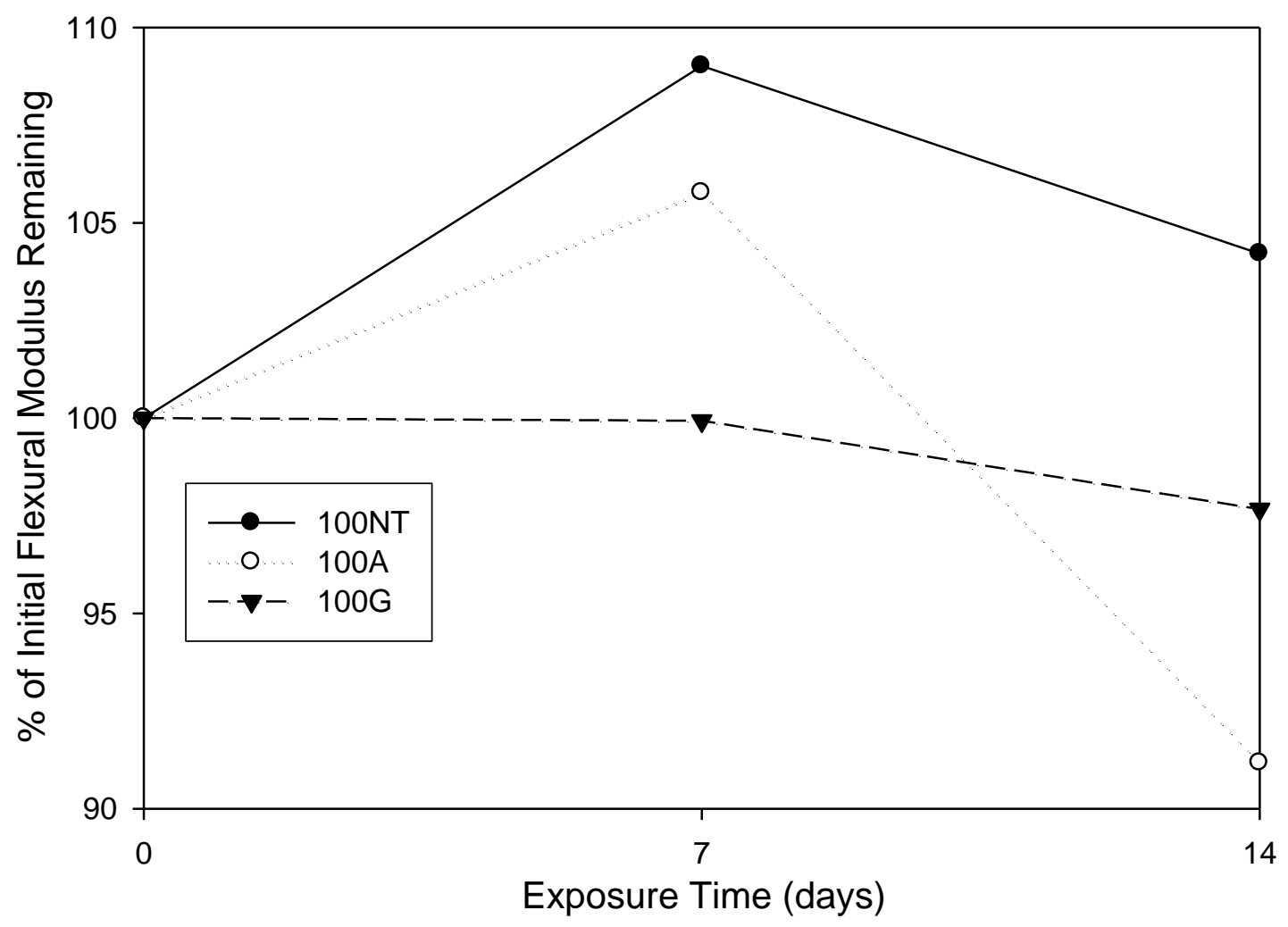

Figure 4.26: Flexural modulus of untreated, annealed and PLA/graphene flexural bars after hydrolysis. 


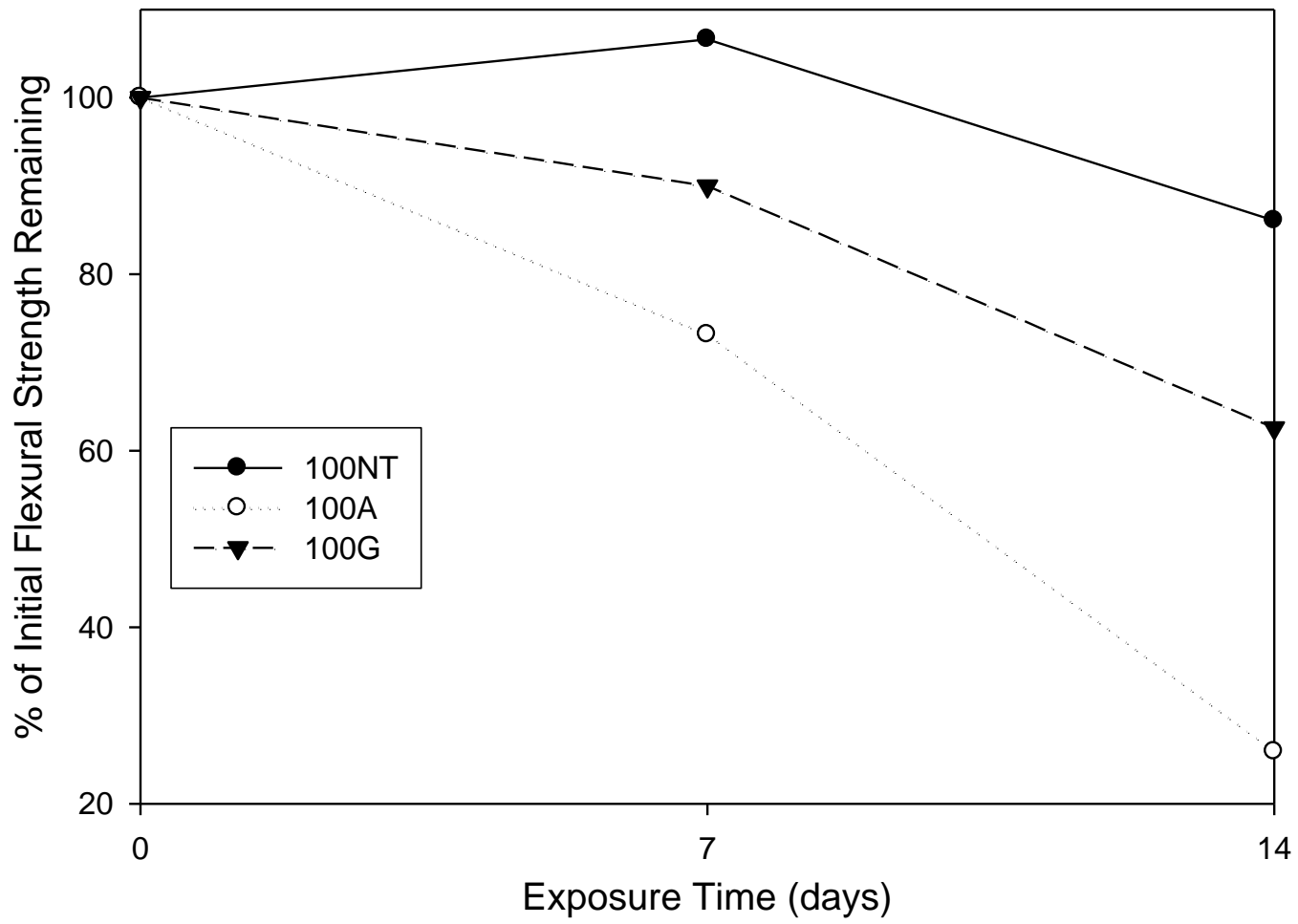

Figure 4.27: Flexural strength of untreated, annealed and PLA/graphene flexural bars after hydrolysis.

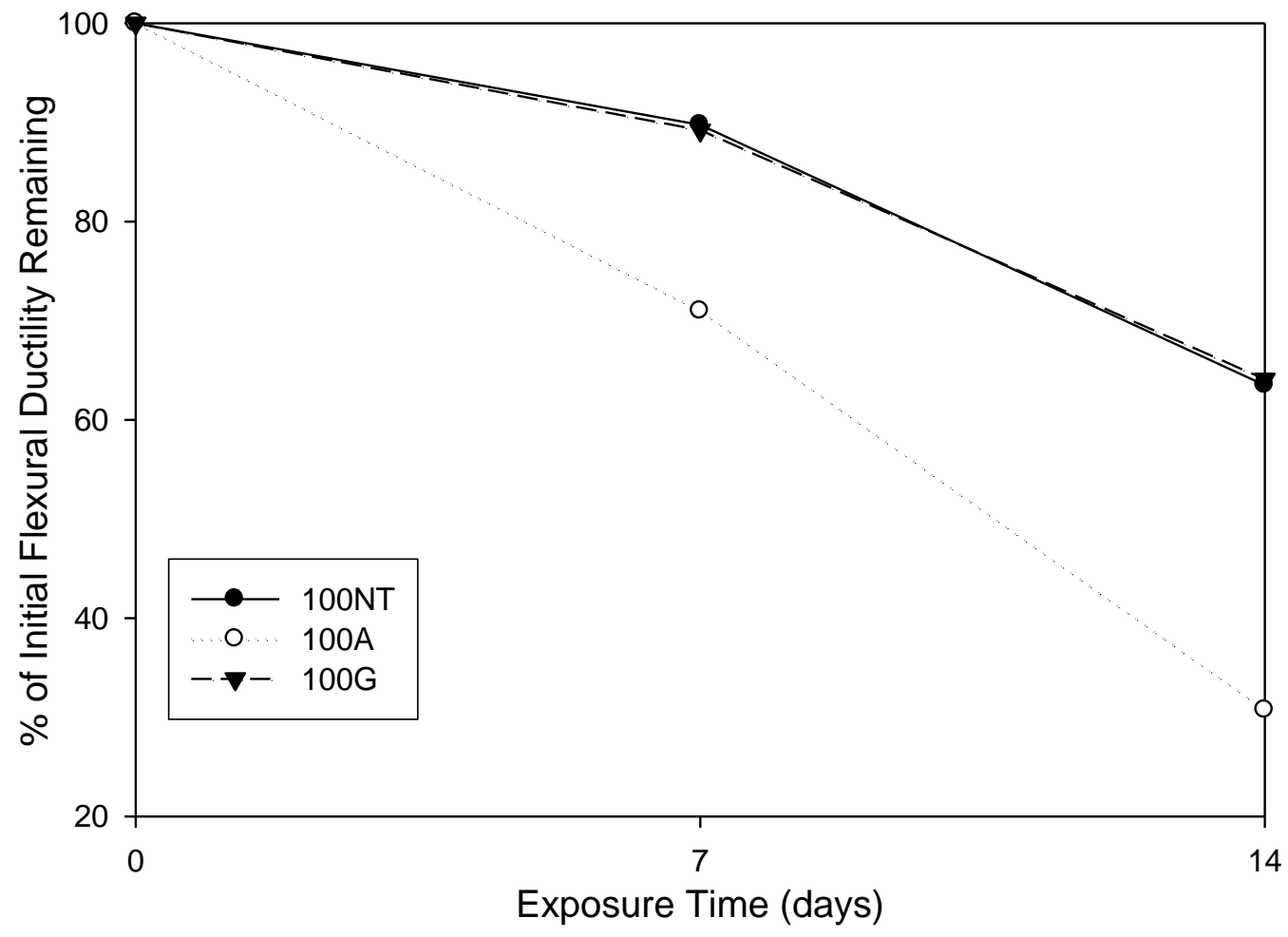

Figure 4.28: Flexural ductility of untreated, annealed and PLA/graphene flexural bars after hydrolysis. 
From these figures, we can see that the modulus is not greatly influenced by the aging process, as it is a low strain property. However, the flexural strength and ductility are by their definition measured at high strain near the failure point and therefore can give a more accurate representation of the damage done during hydrolysis. Here, the treated materials experience greater strength and ductility loss than the untreated; this is likely due to the fact that the treated samples were more brittle to begin with. Because the untreated sample had an amorphous character without the addition of possibly aggregated graphene clusters, it could undergo some degree of hydrolysis before becoming unacceptably brittle. Another interesting result appears if a comparison is made between the viscosity and flexural property losses; by 14 days exposure, the panel's viscosity been reduced by roughly $90 \%$ but the reduction in strength and ductility is about $75 \%$ in the worst case. This seems to suggest that about 14 days exposure (or 4 months outdoor equivalent) is sufficient to damage the molded PLA-only specimens to an unacceptable degree.

However, by testing molded parts we have actually provided a conservative estimate of the materials' hydrolytic stability by adding a thickness-based diffusion resistance. The next step in the investigation is to remove this resistance by testing pellets of the blends under the same hydrolysis conditions.

\subsubsection{Effect of Hydrolytic Degradation on PLA and PLA/Graphene Composites.}

Because pellets of the composites and blends are smaller and thinner than molded pieces, they have a larger specific surface area. This allows attacking media to move more quickly into the bulk of the material without much diffusive resistance, providing a worst-case scenario in terms of degradation. The pellets were degraded like the flexural bars, only in 30 gram batches in aluminum trays laid out in a single level inside the hydrolysis pots rather than in the metal rack. The pellets were then aged for up to 7 days ( 2 months equivalent outdoors) and the melt flow index was measured over this period. Figure 4.29 shows the effect of aging on the absolute value of the MFI, as measured at $250^{\circ} \mathrm{C}$ with $2.16 \mathrm{~kg}$ load for all treatment options: 


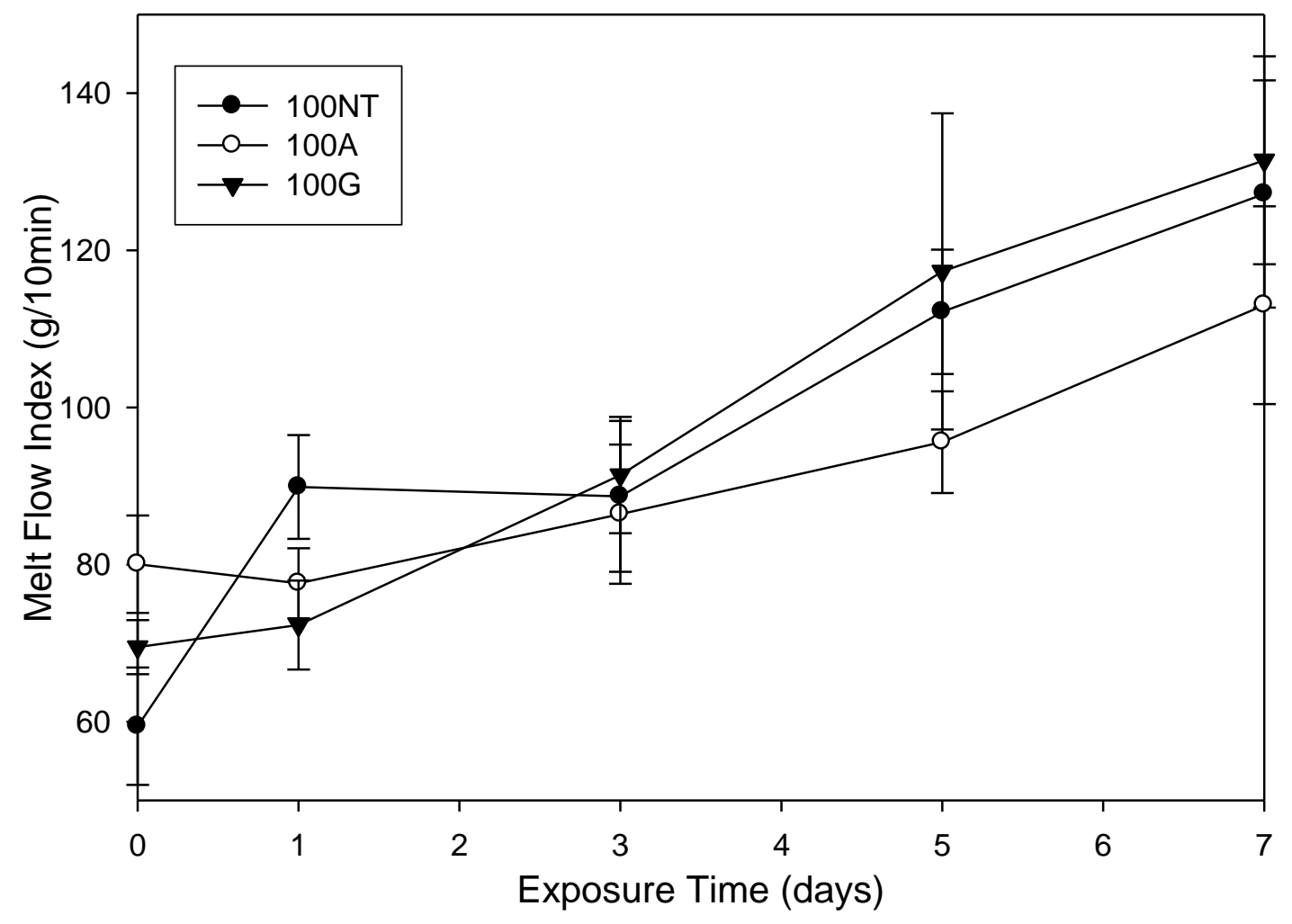

Figure 4.29: Increase in MFI of untreated, annealed, and PLA/graphene materials after hydrolysis.

It is clear from this figure that each material has an initial MFI of roughly $70 \mathrm{~g} / 10 \mathrm{~min}$ which then increases to about $120 \mathrm{~g} / 10 \mathrm{~min}$. We can then plot the increase in melt flow as a percentage in Figure 4.30: 


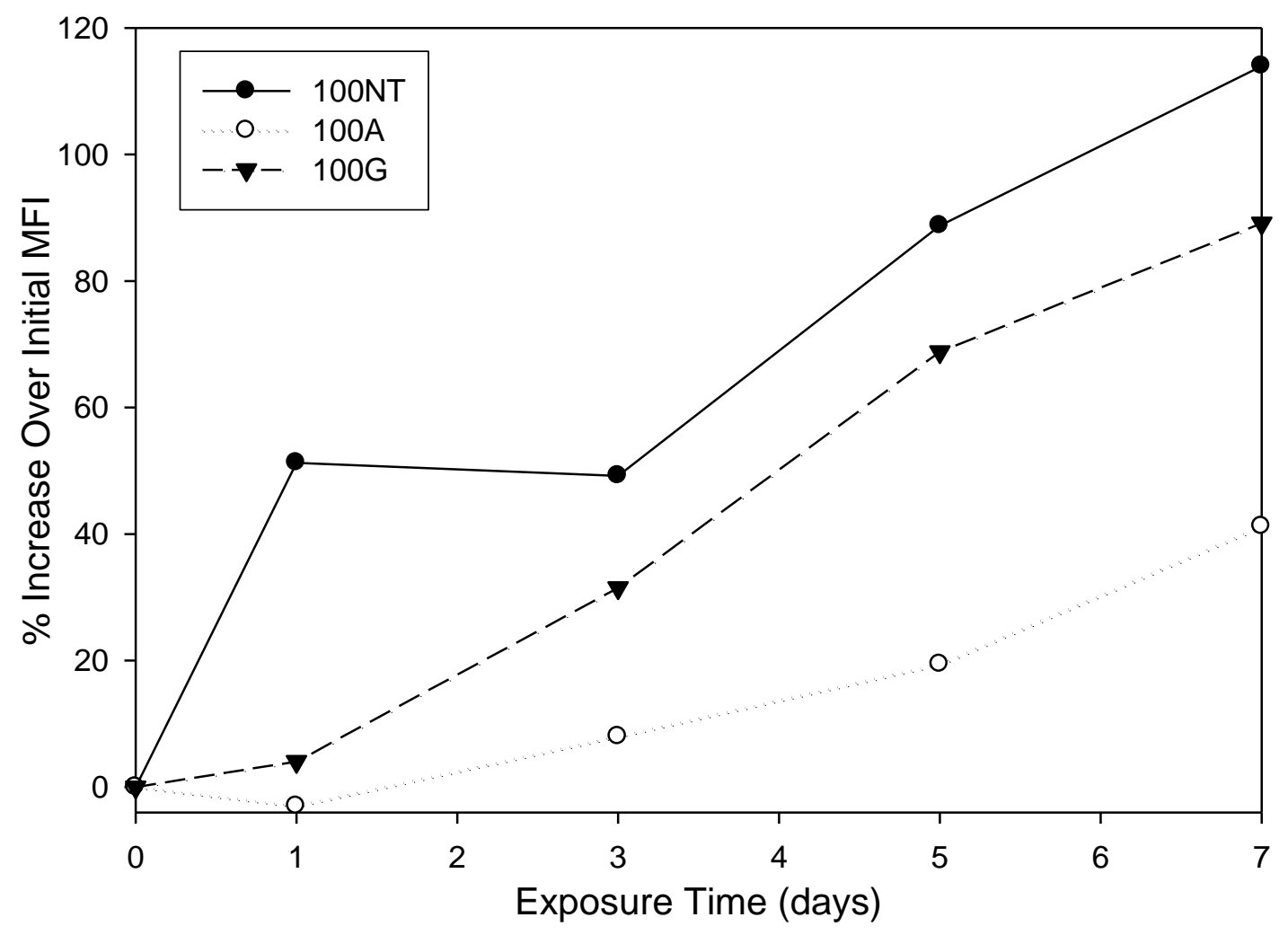

Figure 4.30: Normalized change in MFI for untreated, annealed, and PLA/graphene materials after hydrolysis.

This chart clarifies that in this case, the act of annealing the unfilled PLA pellets creates the best barrier to humidity diffusion. It shows about a $40 \%$ increase in MFI over 7 days exposure compared to about $90 \%$ for the graphene composite and nearly $120 \%$ for the untreated PLA. However, since this only corresponds to 2 months outdoor equivalent aging it would be preferable to see very small increases in the melt flow index. Indeed, if we extrapolate these fairly linear increases (noting that extrapolation outside a data range is dangerous territory!) then by 42 days aging time each sample would experience between a $240-720 \%$ increase in melt flow index depending on the treatment. We next examine some molecular weight measurements that were taken externally at Bayer MaterialScience to quantify the extent to which the materials degraded.

\subsection{Gel Permeation Chromatography (GPC) Results for PLA-only Materials.}

GPC was performed using a Waters Alliance Gel Permeation Chromatograph. Samples were dissolved in methylene chloride, then diluted 50:50 by volume in tetrahydrofuran (THF) and then analyzed in $\mathrm{THF}$ at $35^{\circ} \mathrm{C}$ with a flow rate of $1.0 \mathrm{~mL} / \mathrm{min}$. Molecular weights were determined 
using a calibration curve relative to polystyrene standards. Samples that have not been degraded, along with several that have are shown in the following table:

Table 4.2: GPC results of various PLA and PLA/graphene samples.

\begin{tabular}{||l|c|c|c||}
\hline \multicolumn{1}{|c|}{ Sample } & Mn & Mw & Mw/Mn \\
\hline \hline PLA extruded & 95,200 & 174,250 & 1.83 \\
\hline PLA/graphene & 82,980 & 155,710 & 1.88 \\
\hline $\begin{array}{l}\text { Graphene, 1 } \\
\text { day hydrolysis }\end{array}$ & 77,380 & 150,070 & 1.94 \\
\hline $\begin{array}{l}\text { No treatment , } \\
\text { day } \\
\text { hydrolysis }\end{array}$ & 90,390 & 166,580 & 1.84 \\
\hline $\begin{array}{l}\text { Annealed, 1 } \\
\text { day hydrolysis }\end{array}$ & 81,660 & 151,890 & 1.86 \\
\hline $\begin{array}{l}\text { 3mm pH 7, 1 } \\
\text { week } \\
\text { hydrolysis }\end{array}$ & 50,880 & 102,400 & 2.01 \\
\hline
\end{tabular}

The first two samples are extruded pellets and internally mixed PLA/grapheme pellets with no exposure, therefore these are the initial molecular weights of the materials; as the graphenecontaining samples had a longer mixing time and thermal history the initial molecular weight is slightly lower than the extruded batch. Communications with NatureWorks staff suggest that the initial weight average-molecular weight of PLA 2002D is about 210,000 g/mole so there has not been significant degradation by the chosen processing methods. The next three are pellets of each treatment that were exposed to the hydrolytic chamber for 1 day. The untreated sample had a molecular weight loss of about $8000 \mathrm{~g} /$ mole over this period, while the graphene was slightly lower, indicating that the barrier effect improved stability over this short duration. The final sample was from an untreated $3 \mathrm{~mm}$ thick plaque submerged in distilled water at $50^{\circ} \mathrm{C}$ for one week, so there is molecular weight loss from both the exposure and the second thermal history from molding the plaque. Using the extruded PLA pellet as a benchmark, the plaque had a loss of roughly 70,000 g/mole after 1 week, equivalent to about $7000 \mathrm{~g} / \mathrm{mole} /$ day which is similar to the degradation experienced by the pellets after one day. Harris et al. degraded PLA 3001Dmolded flexural bars in their study and observed a molecular weight loss of roughly 6000 
$\mathrm{g} / \mathrm{mole}_{\mathrm{w}} \mathrm{week}$, for comparison ${ }^{63}$. They also observed that the critical molecular weight below which strength and integrity are lost is about $10,000 \mathrm{~g} / \mathrm{mole}$. This information along with the degradation results leads us to a simple conclusion.

\subsection{Commentary on PLA and PLA/Graphene Durability.}

A high molecular weight, semi-crystalline grade of PLA was employed to test the material's inherent stability for possible usage in the creation of durable engineering plastic goods. Untreated, annealed and graphene-composite samples were fabricated and subjected to a variety of degradation tests. It was determined that the addition of graphene can greatly accelerate the slow crystallization of the PLA, but the dimensions of the crystals formed do not appear to be altered. Both the addition of crystalline domains created by heat treatment and the inclusion of graphene nano-platelets to add diffusion barriers were capable of slowing the rate of degradation. However, long duration tests both in solvents and in a humidity chamber showed the materials to undergo extreme degradation by the equivalent of 1 year outdoor aging in southern Florida. It is logical that the lactic acid monomer contains too high a concentration of vulnerable ester groups to be inherently stable. It is also likely that the rate of diffusion is fast with respect to the rate of the degradation reaction for attacking species through the PLA bulk, which would explain the lack of a significant enhancement in durability by way of retarding diffusion; later we will investigate this claim in more detail. Therefore, the addition of a second, more stable polymer phase in the form of polycarbonate will be investigated by the same techniques.

\subsection{Blends of Polylactic Acid and Polycarbonate.}

We previously mentioned that one of the most common ways to improve a lacking characteristic of a chosen polymer was to blend it with another phase which can make up for that deficiency. In this case, polycarbonate has better mechanical properties and is much more thermally and hydrolytically stable than PLA; therefore, a suite of blends ranging from 20-80\% polycarbonate (including 100\% PC as well) was created and evaluated as before to ascertain the material durability.

\subsubsection{Fabrication of the PC/PLA and PC/PLA/Graphene Composites.}

As was listed in Table 4.1, these blends and composites were compounded in either a twin-screw extruder or an internal mixer for graphene-filled composites. The compounding conditions in either case are listed in Table 4.3: 
Table 4.3: PC/PLA and PC/PLA/Graphene experimental materials and method of production.

\begin{tabular}{|l|r|r|r|l|c|}
\hline Sample & \% PLA & \% PC & \% Graphene & Method & Mixing Conditions \\
\hline $80 N T$ & 80 & 20 & 0 & extrusion & see Table 3.1 \\
\hline $80 A$ & 80 & 20 & 0 & extrusion & see Table 3.1 \\
\hline $80 G$ & 79 & 19 & 2 & int mix & $240^{\circ} \mathrm{C}-80 \mathrm{rpm}-5 \mathrm{~min}$ \\
\hline $60 N T$ & 60 & 40 & 0 & extrusion & see Table 3.1 \\
\hline $60 A$ & 60 & 40 & 0 & extrusion & see Table 3.1 \\
\hline $60 G$ & 59 & 39 & 2 & int mix & $240^{\circ} \mathrm{C}-80 \mathrm{rpm}-5 \mathrm{~min}$ \\
\hline $40 N T$ & 40 & 60 & 0 & extrusion & see Table 3.1 \\
\hline $40 A$ & 40 & 60 & 0 & extrusion & see Table 3.1 \\
\hline $40 G$ & 39 & 59 & 2 & int mix & $240^{\circ} \mathrm{C}-80 \mathrm{rpm}-5 \mathrm{~min}$ \\
\hline $40 A G$ & 39 & 59 & 2 & int mix & $240^{\circ} \mathrm{C}-80 \mathrm{rpm}-5 \mathrm{~min}$ \\
\hline $30 N T$ & 30 & 70 & 0 & extrusion & see Table 3.1 \\
\hline $30 A$ & 30 & 70 & 0 & extrusion & see Table 3.1 \\
\hline $30 G$ & 29 & 69 & 2 & int mix & $240^{\circ} \mathrm{C}-80 \mathrm{rpm}-5 \mathrm{~min}$ \\
\hline $30 A G$ & 29 & 69 & 2 & int mix & $240^{\circ} \mathrm{C}-80 \mathrm{rpm}-5 \mathrm{~min}$ \\
\hline $20 N T$ & 20 & 80 & 0 & extrusion & see Table 3.1 \\
\hline $20 A$ & 20 & 80 & 0 & extrusion & see Table 3.1 \\
\hline $20 G$ & 19 & 79 & 2 & int mix & $240^{\circ} \mathrm{C}-80 \mathrm{rpm}-5 \mathrm{~min}$ \\
\hline $20 A G$ & 19 & 79 & 2 & int mix & $240^{\circ} \mathrm{C}-80 \mathrm{rpm}-5 \mathrm{~min}$ \\
\hline$O N T$ & 0 & 100 & 0 & extrusion & see Table 3.1 \\
\hline$O G$ & 0 & 98 & 2 & int mix & $240^{\circ} \mathrm{C}-80 \mathrm{rpm}-5 \mathrm{~min}$ \\
\hline
\end{tabular}

Here, the same nomenclature applies as it did with Table 4.1. In this case, samples that were PCrich had an additional treatment of annealing with grapheme and were labeled as $A G$; this was not performed with PLA-rich samples as these were extremely brittle. Once more, the extrusion conditions are listed in Chapter 3 for each composition to achieve a roughly constant $70 \%$ torque for each.

Cryo-fractured plaques of each of these multi-phase materials was investigated with SEM to discern if the processing methods produced morphologies with acceptable distribution and dispersion of the minor phase and additives. In this case as before, samples that have been annealed have not been included as this is not expected to alter the droplet shape or dispersion. These pictures are listed as Figures 4.31-4.36: 

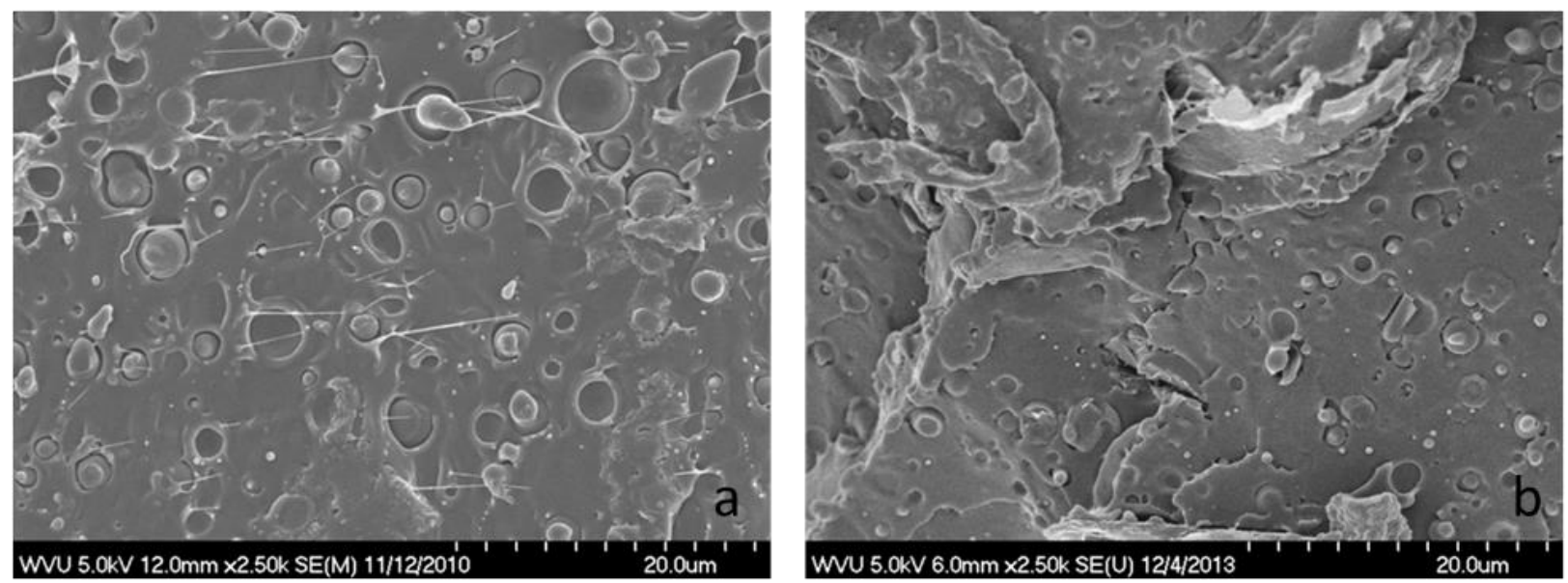

Figure 4.31: Images of blends $80 N T$ (a) and $80 G$ (b).
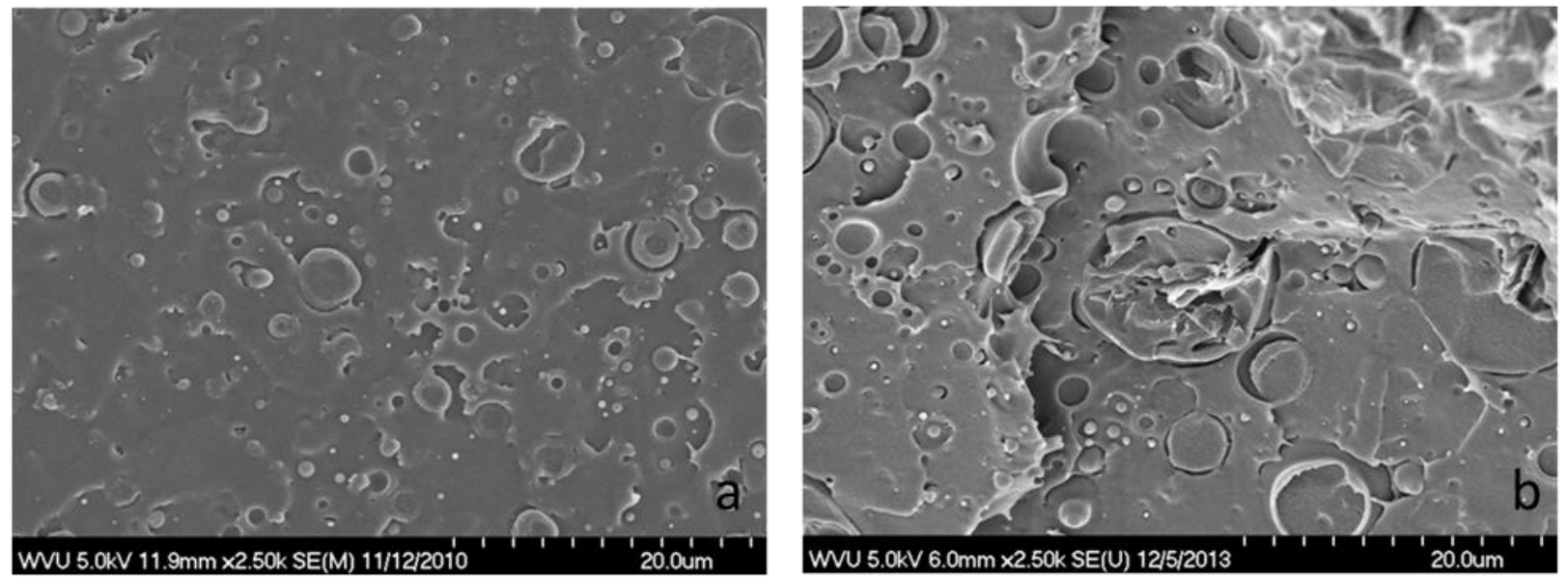

Figure 4.32: Images of blends $60 N T$ (a) and $60 G$ (b).
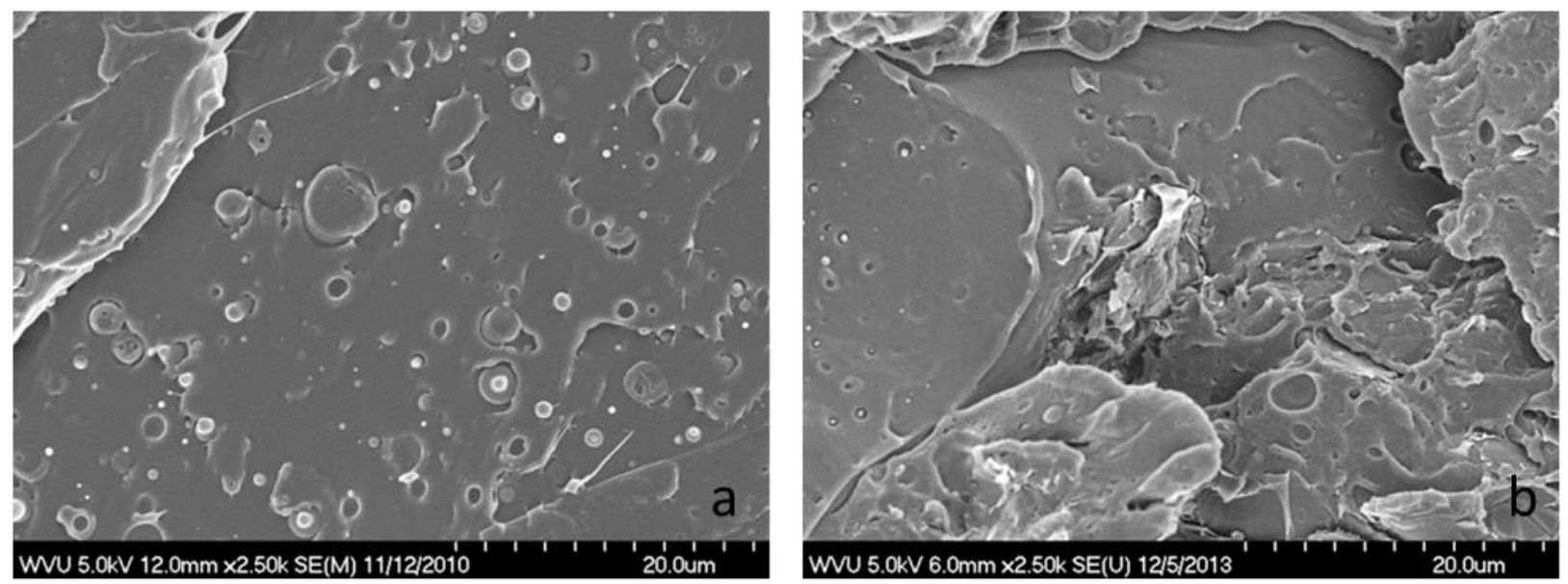

Figure 4.33: Images of blends $40 N T$ (a) and $40 G$ (b). 

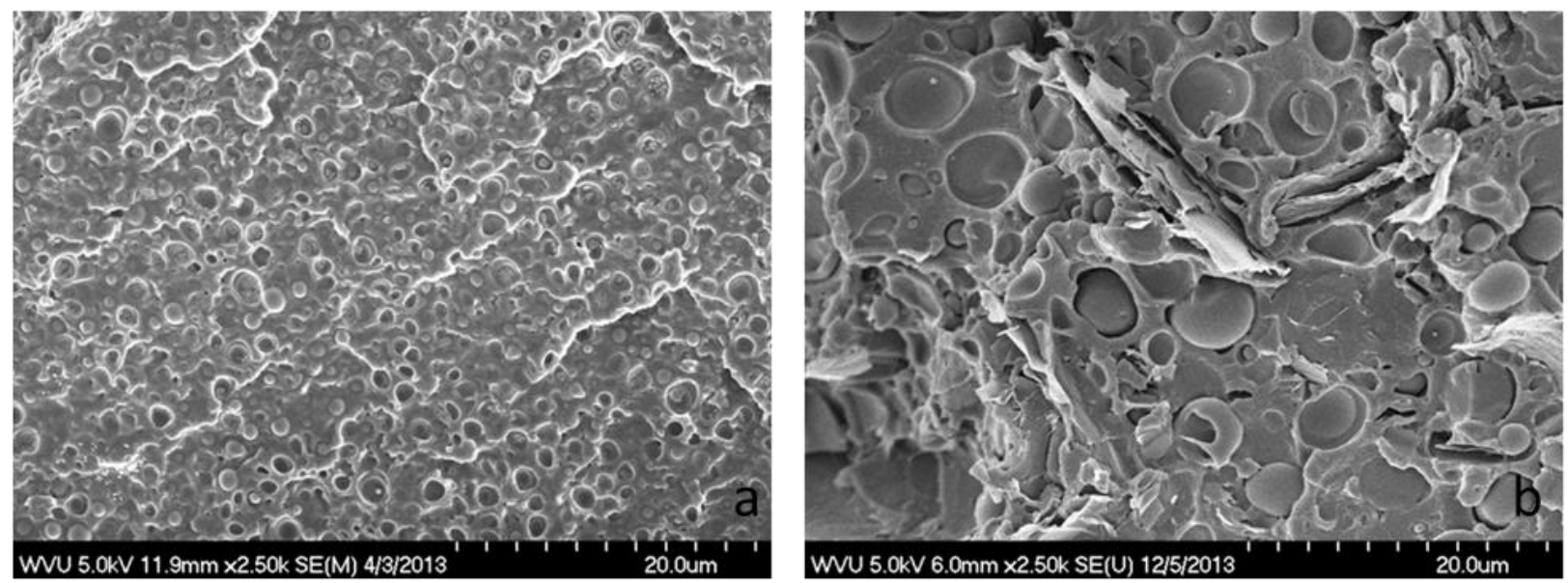

Figure 4.34: Images of blends $30 N T$ (a) and $30 G$ (b).
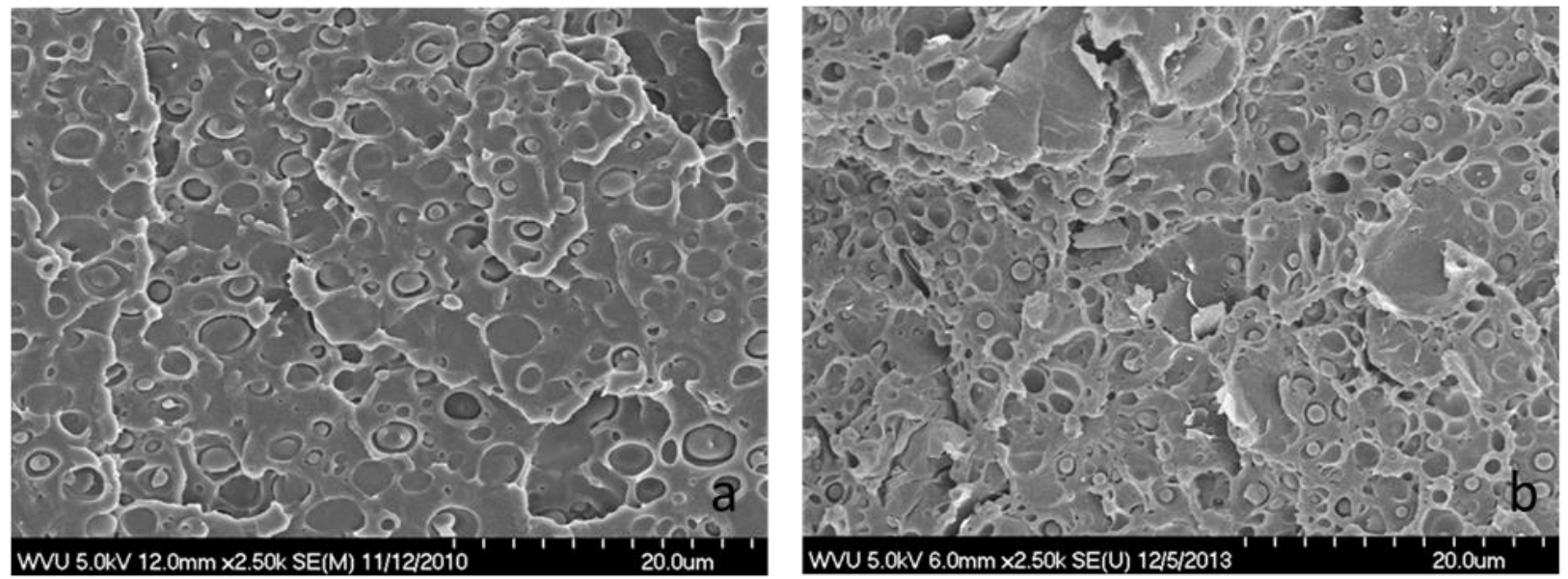

Figure 4.35: Images of blends 20NT (a) and 20G (b).
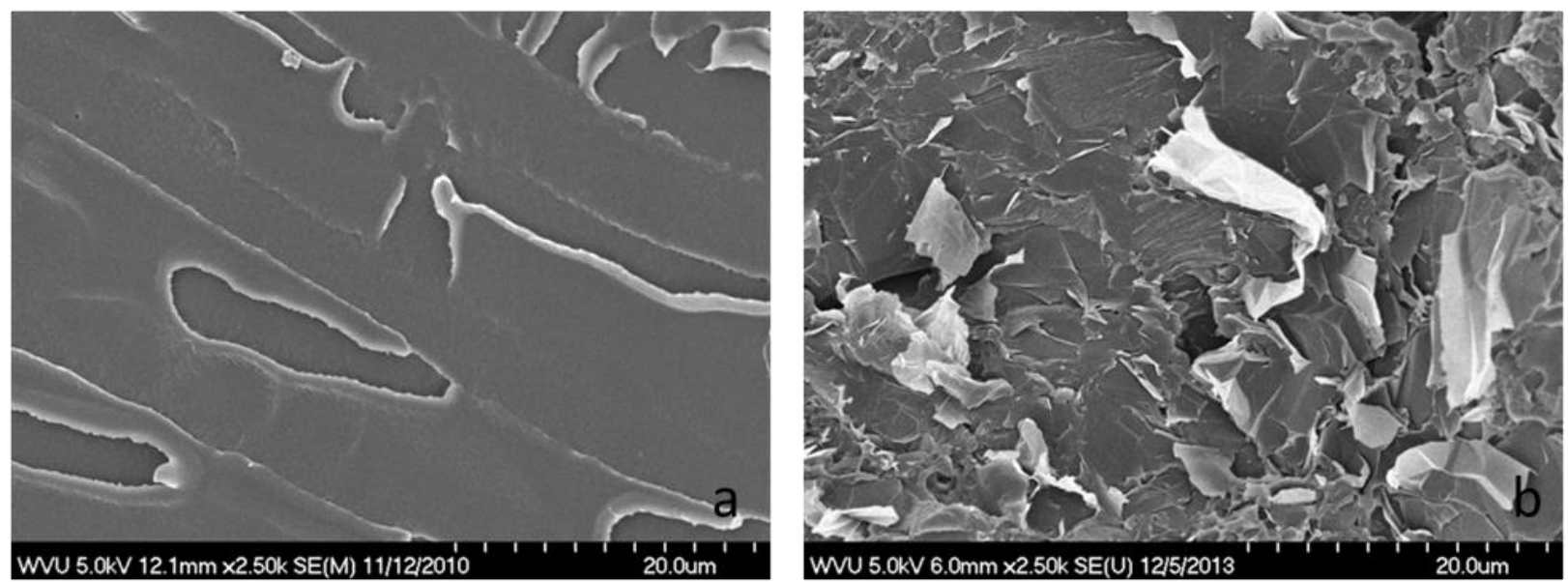

Figure 4.36: Images of blends $O N T$ (a) and $O G(\mathrm{~b})$.

All images are shown at 2500 times magnification, where the scale bar denotes a $20.0 \mu \mathrm{m}$ length. There are different structures present in the two-phase polymer blends, where the PLA-rich 
blends have needle-like and droplet images moving but only droplets are present for blends that are rich in polycarbonate; the needles may be some type of long-range crystalline PLA domains. It is also clear that the two polymers are immiscible, as there is a pull-away at the interface. In terms of composition, images 4.32-33 seem to form roughly co-continuous matrices; this is not uncommon for blends with two phases that are roughly equal in weight (e.g. ${ }^{97}$ ). The clearly matrix-dispersed images with 80, 30, and $20 \%$ weight PLA are slightly different from each other in that the PC-rich materials have very fine morphologies but the 20/80 PC/PLA blend does not; this is likely a function of the viscosity ratio, where the highly viscous PC matrix can easily transfer stress and mechanical energy to the low viscosity dispersed phase PLA to break it up, but the reverse may not hold true. Whatever the case, image analysis was performed on the above images to quantify the particle sizes when possible; the ImageJ software originally developed by NIH researchers was used. At least 100 particles were sized wherever possible and varying particle size calculations were performed using the following equation ${ }^{98}$ :

$$
\bar{D}_{p q}^{(p-q)}=\frac{\sum_{i} D_{i}^{p}}{\sum_{i} D_{i}^{q}}
$$

In this case, the number average $d_{10}$ and the so-called DeBroukere average $d_{43}$ particle diameters were of interest. The DeBroukere or Herdan diameter has been described in the literature as the mean diameter over volume ${ }^{98}$. The particles sizes are listed in Table 4.4:

Table 4.4: Particle size analysis of PC/PLA and PC/PLA/graphene composites.

\begin{tabular}{|c|c|c|}
\hline Sample & dB avg diam. d43 $(\boldsymbol{\mu m})$ & num. avg diam. d $\mathbf{1 0}(\boldsymbol{\mu m})$ \\
\hline $80 N T$ & 2.98 & 1.96 \\
\hline $80 G$ & 2.10 & 1.21 \\
\hline $60 N T$ & 4.51 & 1.85 \\
\hline $60 G^{*}$ & 4.32 & 1.22 \\
\hline $40 N T$ & 2.88 & 1.27 \\
\hline $40 G$ & 1.77 & 0.89 \\
\hline $30 N T$ & 1.31 & 0.93 \\
\hline $30 G$ & 4.53 & 2.22 \\
\hline $20 N T$ & 2.35 & 1.60 \\
\hline $20 G$ & 1.52 & 1.14 \\
\hline
\end{tabular}

The sample $60 G$ did not have 100 easily discernable particles to size, so only 94 were included in that calculation. It appears in most cases there is a slight decrease in particle sizes between a 
given composition and the graphene-containing version; this is probably due to extra shearing and mixing action due to the additive. However, the large, almost blade-like graphene structures present seem to confirm that these are aggregates rather than fully exfoliated platelets; this again is common for polymer nano-composites as conventional processing does not seem to be adequate to break up the graphene layers. Lastly, these numbers are in no way perfectly indicative of the actual particle sizes and size distributions of the morphology of a molded part but only a qualitative estimate. For all intents and purposes, however, it seems that the morphologies of all blends and composites studied have particles on the order of 1-5 microns with good dispersion and that the graphene remains in a somewhat aggregated state.

We will now address the crystallization of the PC/PLA blends and composites to determine if the addition of another polymer phase alters the kinetics or dimensionality of crystal growth.

\subsubsection{Crystallization of the PC/PLA and PC/PLA/Graphene Composites.}

The blends were subjected to the same crystallization procedure as the PLA-only materials to obtain the crystallization kinetics. The Avrami exponent and kinetic parameters are now plotted in Figures 4.37-38, again from the primary crystallization regime over which $5-20 \%$ conversion has taken place: 


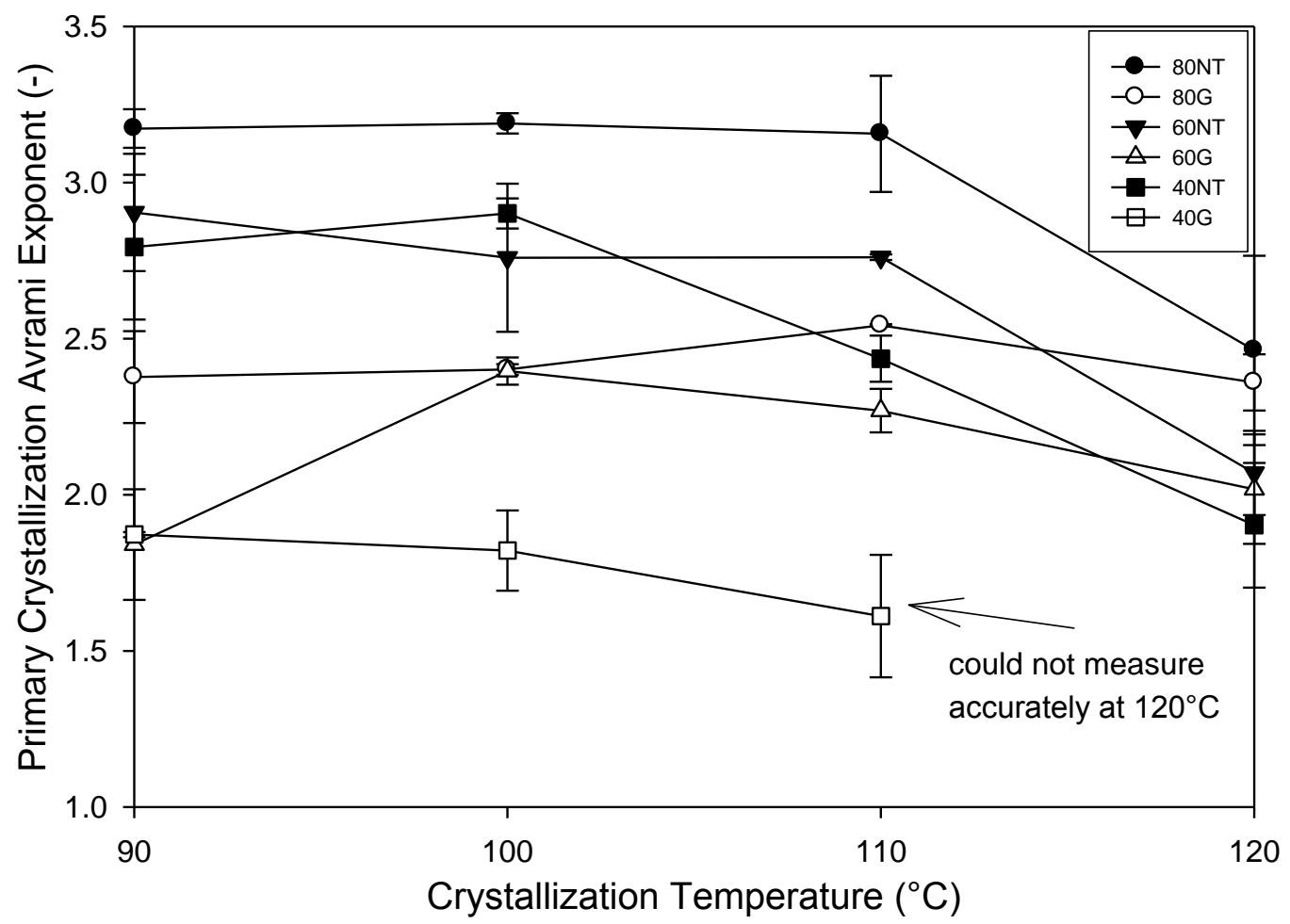

Figure 4.37: Avrami exponent for primary crystallization of PC/PLA and PC/PLA/graphene blends.

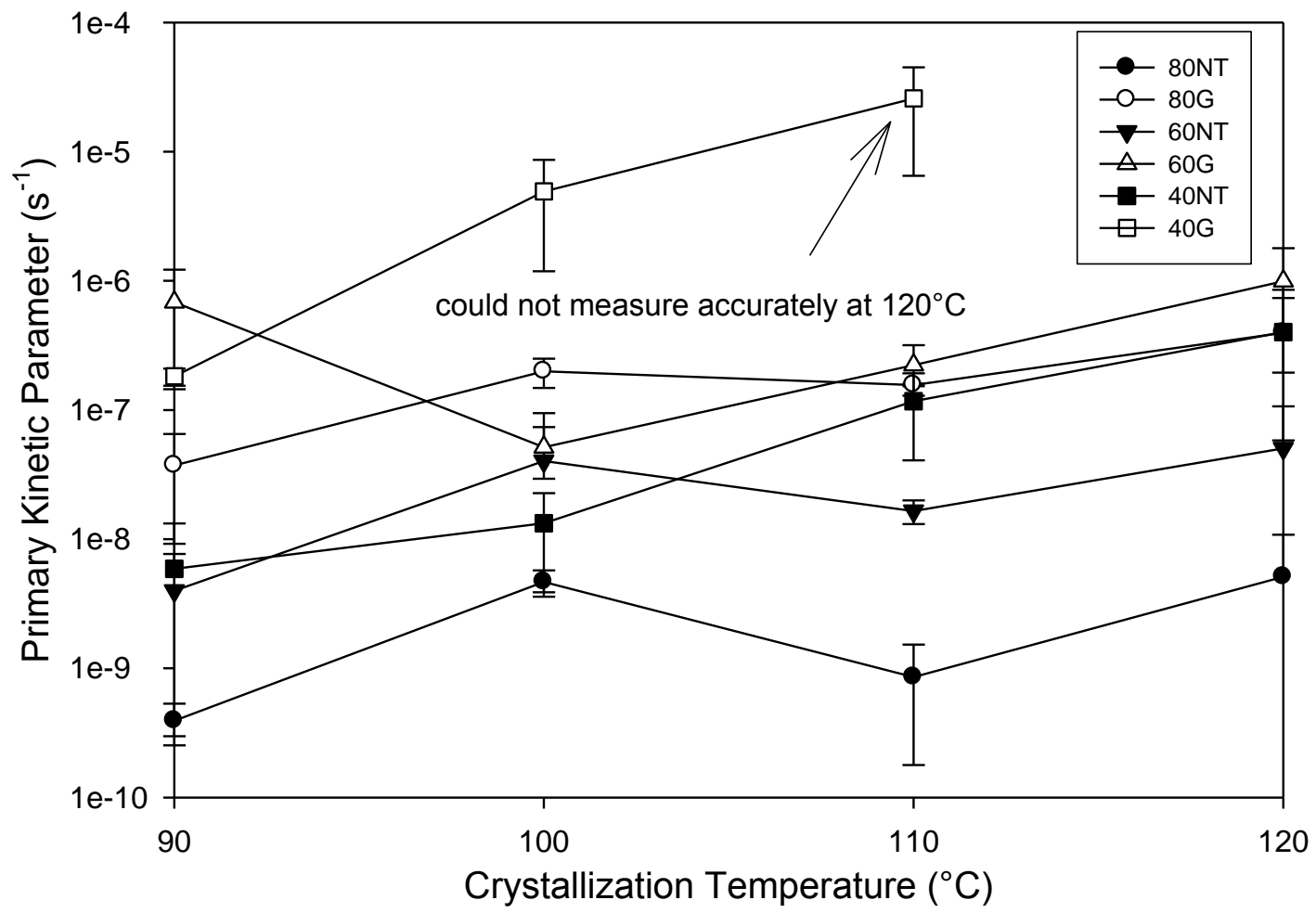

Figure 4.38: Kinetic parameter for primary crystallization of PC/PLA and PC/PLA/graphene blends. 
Samples containing less than $\sim 40 \%$ PLA content gave crystallization data that was inconsistent and very difficult to analyze, although dynamic DSC thermograms were still of use. Additionally, the data for sample $40 G$ crystallized at $120^{\circ} \mathrm{C}$ were also essentially noise even after multiple replications; the data at lower temperatures were normal so it is not clear why this result was obtained. However, we can see from the figures that as graphene is added to the mixture, the Avrami exponent shifts lower from about 3 for the unfilled samples to 2.5 or lower still for the $40 \%$ PLA sample with graphene. This is sensible as theory tells us that an imperfection or additive that acts as a nucleation site would decrease the exponent as it is comprised of both a shape and nucleation term, and the nucleation term is 0 for heterogeneous and 1 if homogeneous. The exponent is also roughly constant across the temperature range for each composition until $120^{\circ} \mathrm{C}$, where it again decreases. This means that crystals grown at the higher temperature have a different shape than those grown between $90-110^{\circ} \mathrm{C}$; unpublished preliminary results showed that no discernable crystallization occurred during experiments at $130^{\circ} \mathrm{C}$ so this may be near the upper temperature limit where PLA crystals can exist. From Figure 4.38, comparing the open marks to the filled, one can observe the increase in the kinetic parameter when graphene is present, though the effect is not quite as profound as for the PLA-only materials (see Figure 4.7). In spite of the effects of graphene, it does not appear that the presence of polycarbonate in these compositions affects the crystal growth or kinetics. This may not be universally true, however; as mentioned above, the highly PC-rich samples gave inconsistent data which may be due to interference by the PC matrix.

With the effects of graphene and the inclusion of polycarbonate on PLA crystallization known, it is again time to examine the effects of these variables on the properties of the composites.

\subsubsection{Initial Crystallinity of PC/PLA and PC/PLA/Graphene Composites.}

As before, the samples were heat treated at $80^{\circ} \mathrm{C}$ for 48 hours in an oven without convection to produce crystallinity and to have the same thermal history. The initial crystallinities of the PC/PLA and PC/PLA/graphene blends in both pellet and molded part form are shown in Figures 4.39-40: 


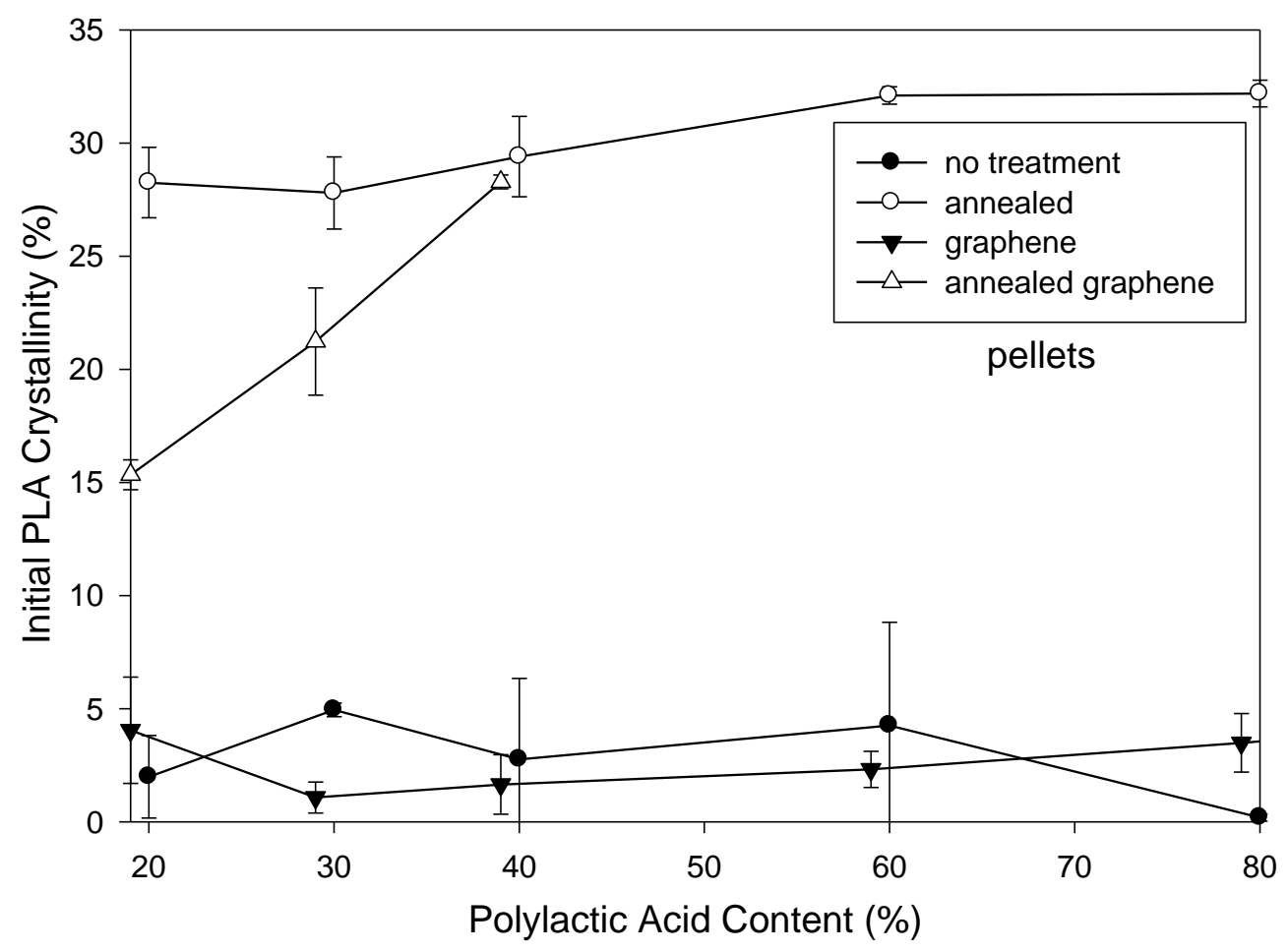

Figure 4.39: Initial crystallinity of PC/PLA and PC/PLA/graphene pellet samples.

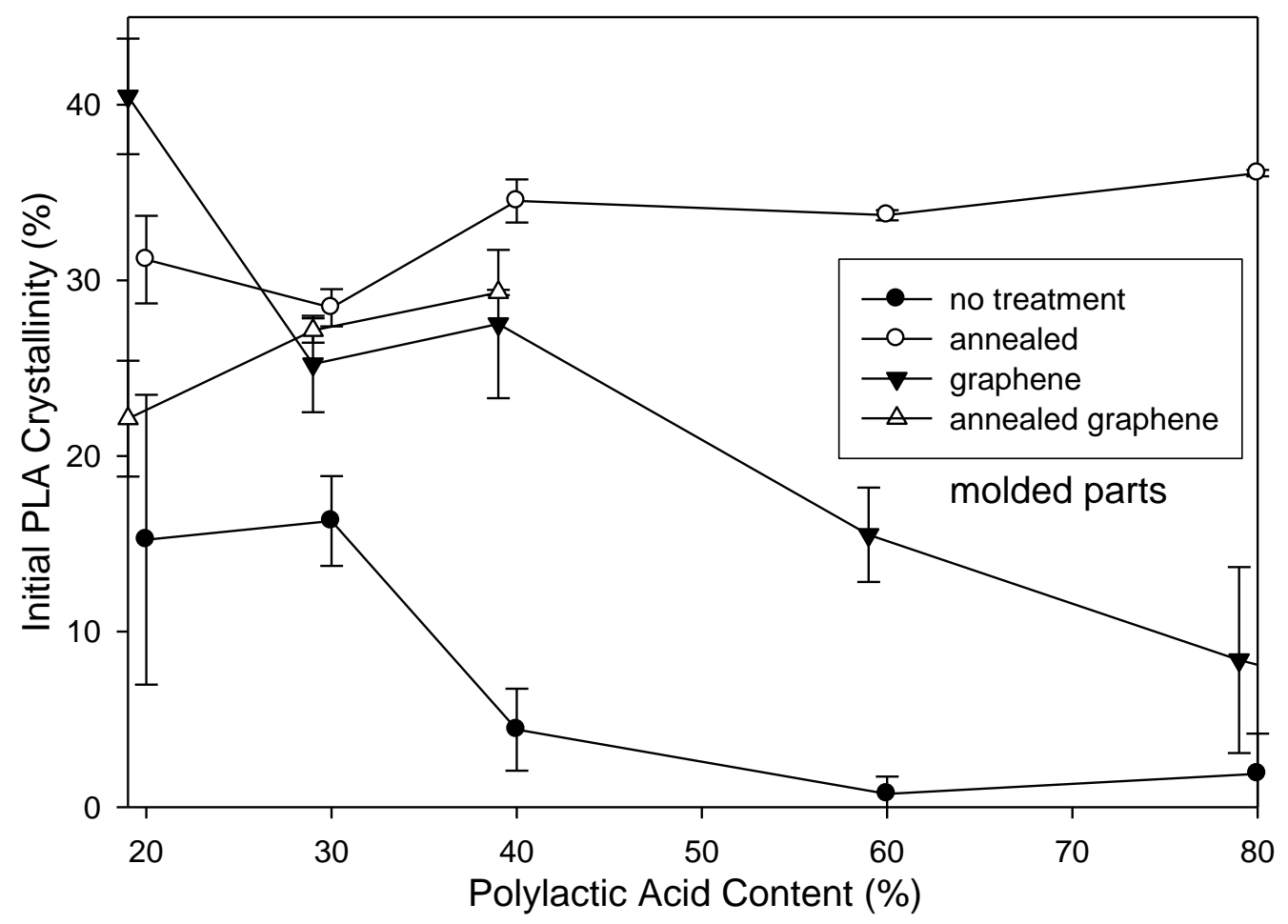

Figure 4.40: Initial crystallinity of PC/PLA and PC/PLA/graphene molded parts. 
As listed in Table 4.3, the samples that contained nominally 20-40\% PLA phase had the additional treatment of annealing plus graphene. Here, the pellets without any heat treatment were essentially amorphous and those that were annealed had near the maximum of $30 \%$ crystallinity. For annealed/graphene pellets, the crystallinity increased as the PLA content increased rather than being a constant near 30\%; the nucleating effect of graphene in the PLA phase may explain this observation. For the molded parts, the annealed samples act as expected, though other compositions are a bit different. The graphene-containing samples that are PC-rich have a much higher crystallinity than the pellets, which may come about from nucleating effects or from the second thermal history required to mold the parts. The untreated and the annealed/graphene samples have similar crystallinities to the pellets.

Now that the initial crystalline properties are known, that effect on hydrolytic stability may be observed.

\subsubsection{Solvent Degradation of PC/PLA and PC/PLA/Graphene Composites.}

The untreated, annealed, and graphene-containing blends of varying PC/PLA compositions were subjected to the solvent degradation regime at $50^{\circ} \mathrm{C}$. The change in weight of the untreated PC/PLA molded panels after exposure to the $\mathrm{pH} 10$ carbonate buffer is given in Figure 4.41: 


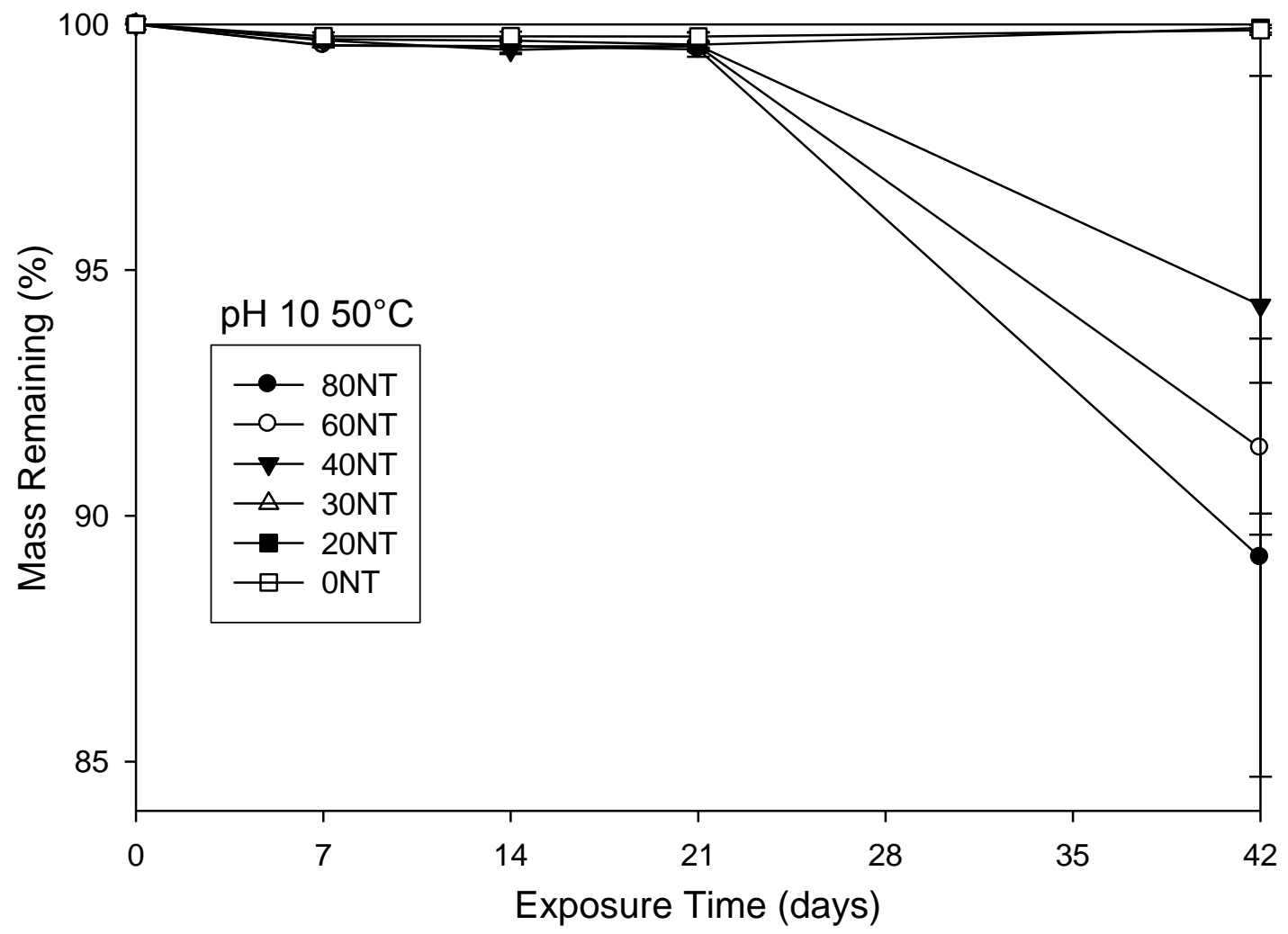

Figure 4.41: Weight loss of untreated PC/PLA molded panels during exposure to carbonate buffer.

Here, it is clear that the parts that were increasingly PLA-rich lost increasing amounts of mass, as the PC phase is much more stable; we can recall Figure 4.12 and see that sample 100NT lost about $16 \%$ of its mass for comparison. Further, this set of data is the only one besides the 98 $100 \%$ PLA panels to show any weight loss; this seems to suggest that the heat and graphene treatments provided enough of a barrier to diffusion that only bulk degradation took place. This also confirms the PLA phase is susceptible to highly basic solutions and that even with polycarbonate present, there must be enough PC phase so that the vulnerable PLA is encapsulated to protect it.

The next step in evaluating the durability of the PC/PLA blends and composites is to observe the change in viscosity after degradation has taken place. The rheological plots in Figures 4.42-44 are for plaques of all treatment options of the blend containing 79-80\% PLA content: 


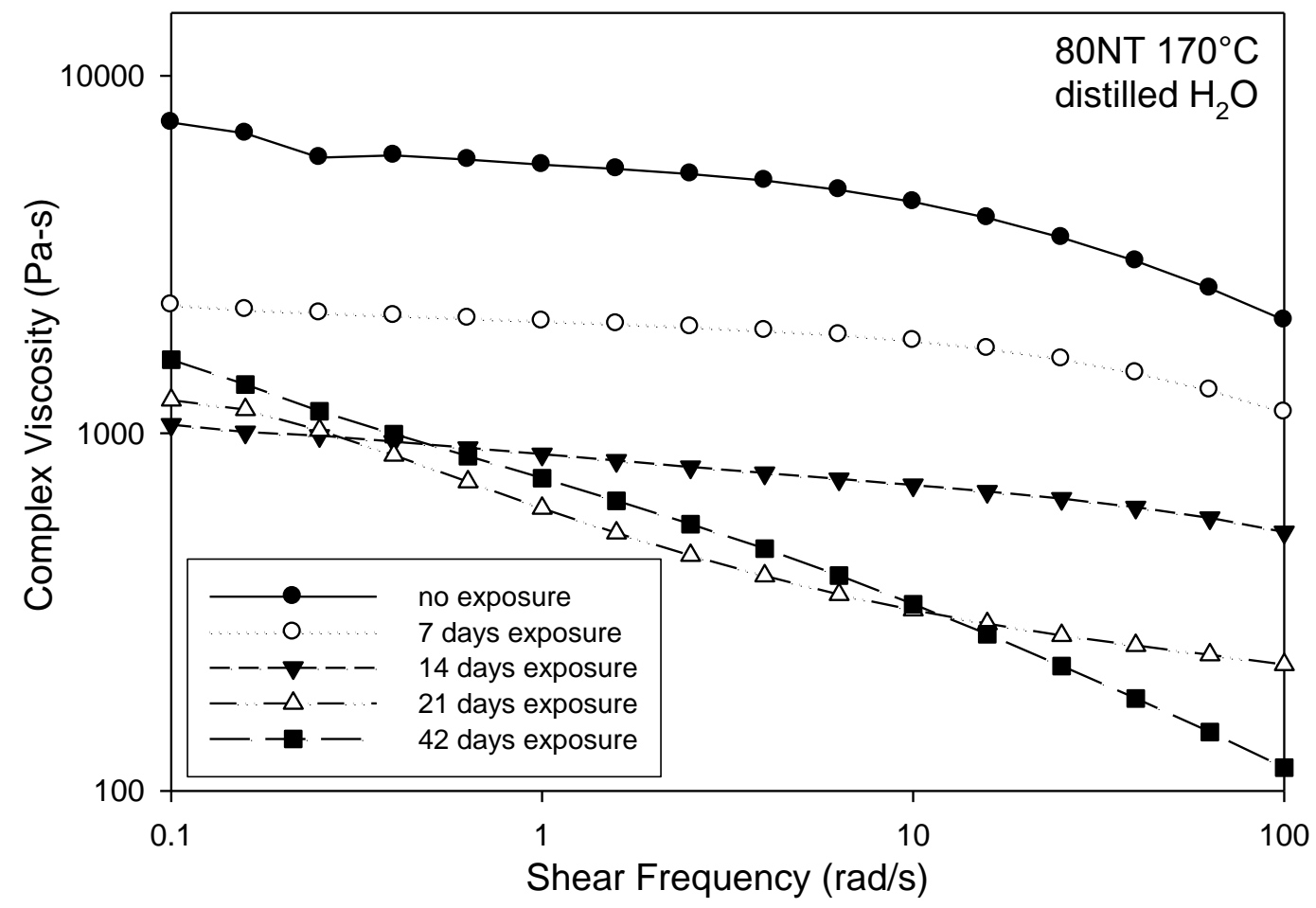

Figure 4.42: Viscosity change of untreated $80 \%$ PLA panels after exposure to distilled water.

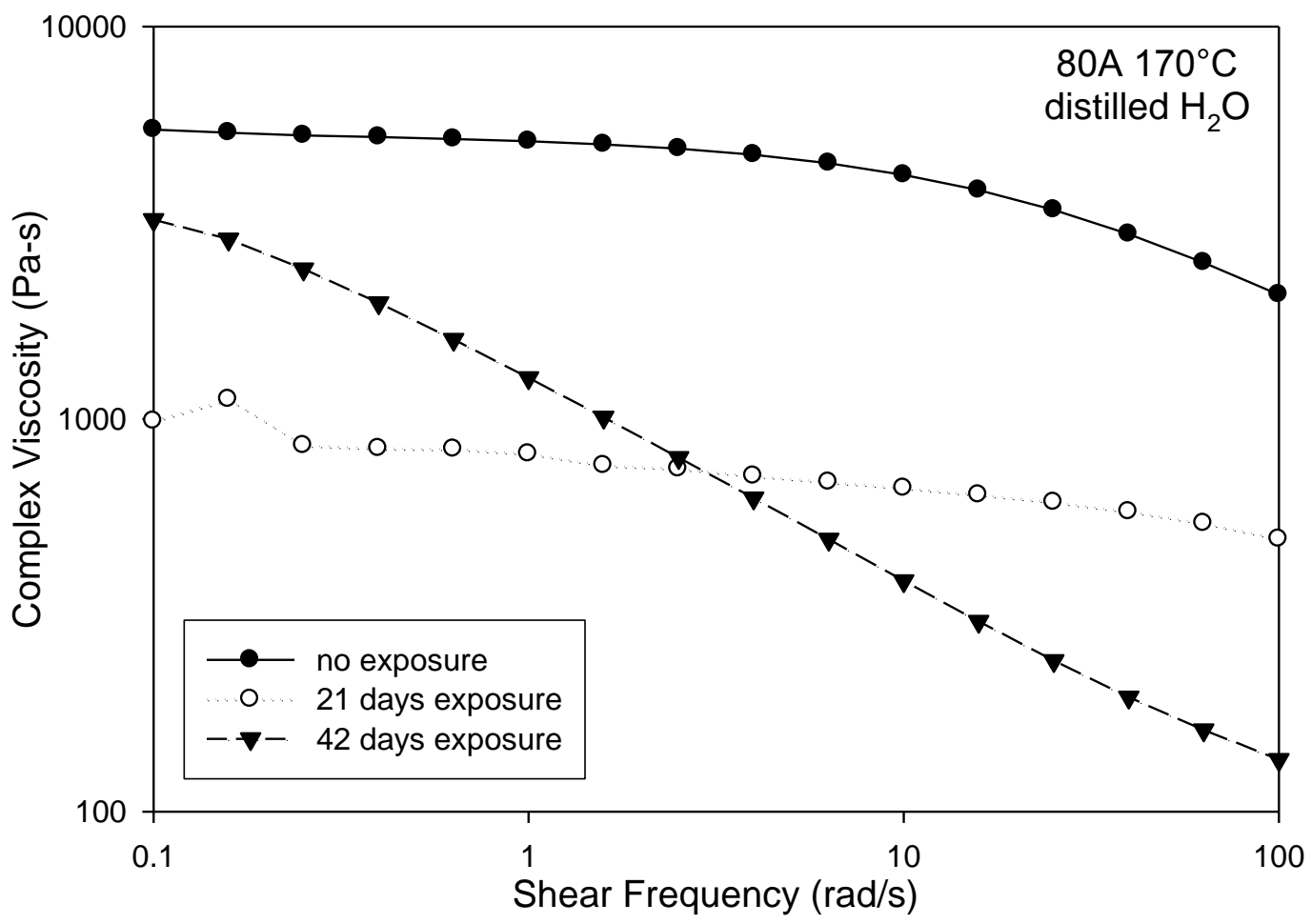

Figure 4.43: Viscosity change of untreated $80 \%$ PLA panels after exposure to distilled water. 


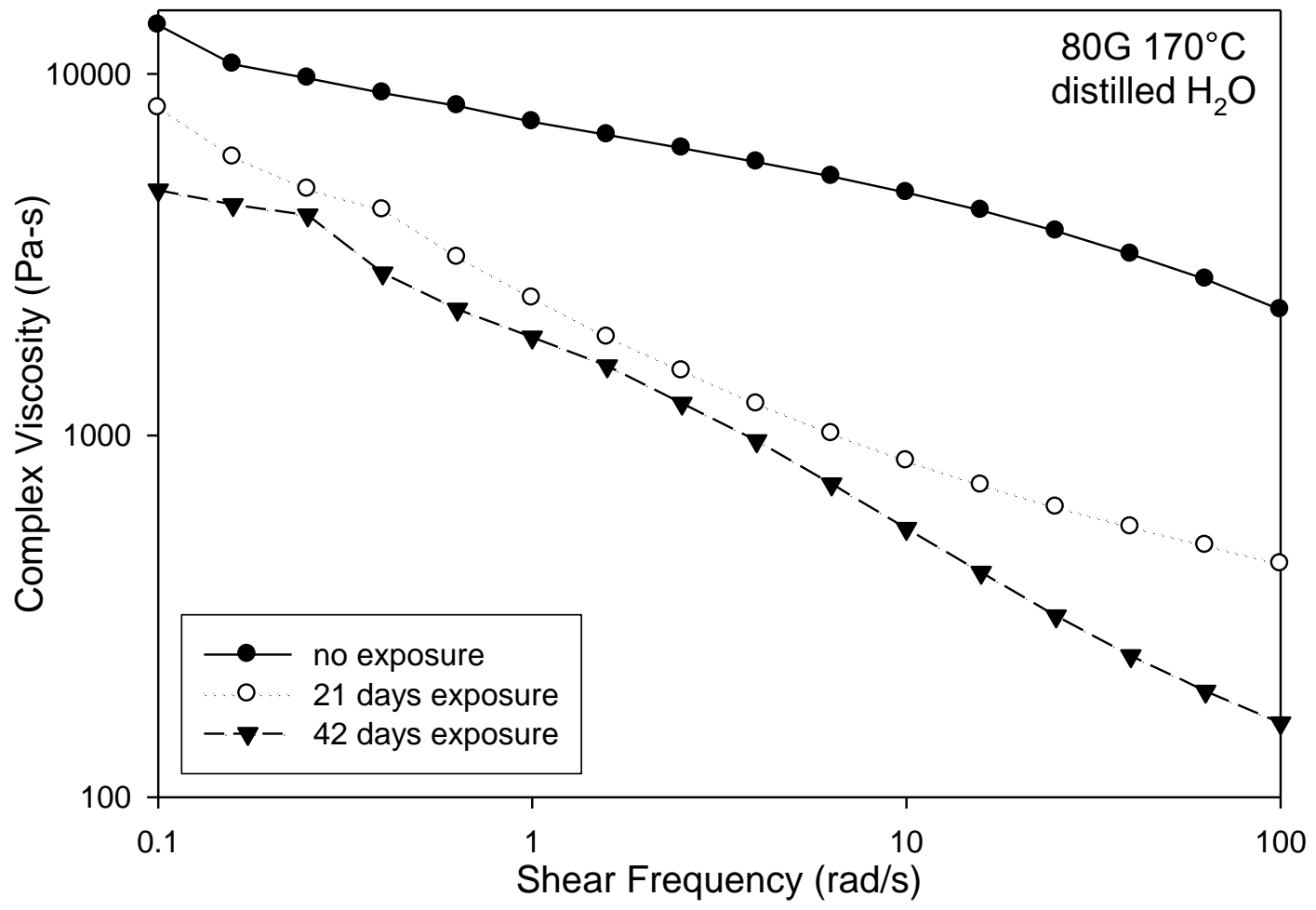

Figure 4.44: Viscosity change of 79\% PLA/graphene panels after exposure to distilled water.

Unlike the viscosity loss curves shown for the 98-100\% PLA blends with different treatments, these materials have retained the shear-thinning characteristic of polymeric melts. Further, we can see that while appreciable degradation has taken place over the 42 days of aging, the materials have not been completely destroyed like those containing only PLA. The next step is to again show the plots of each treatment at $1 \mathrm{rad} / \mathrm{second}$ to compare the effect of each treatment option in Figure 4.45: 


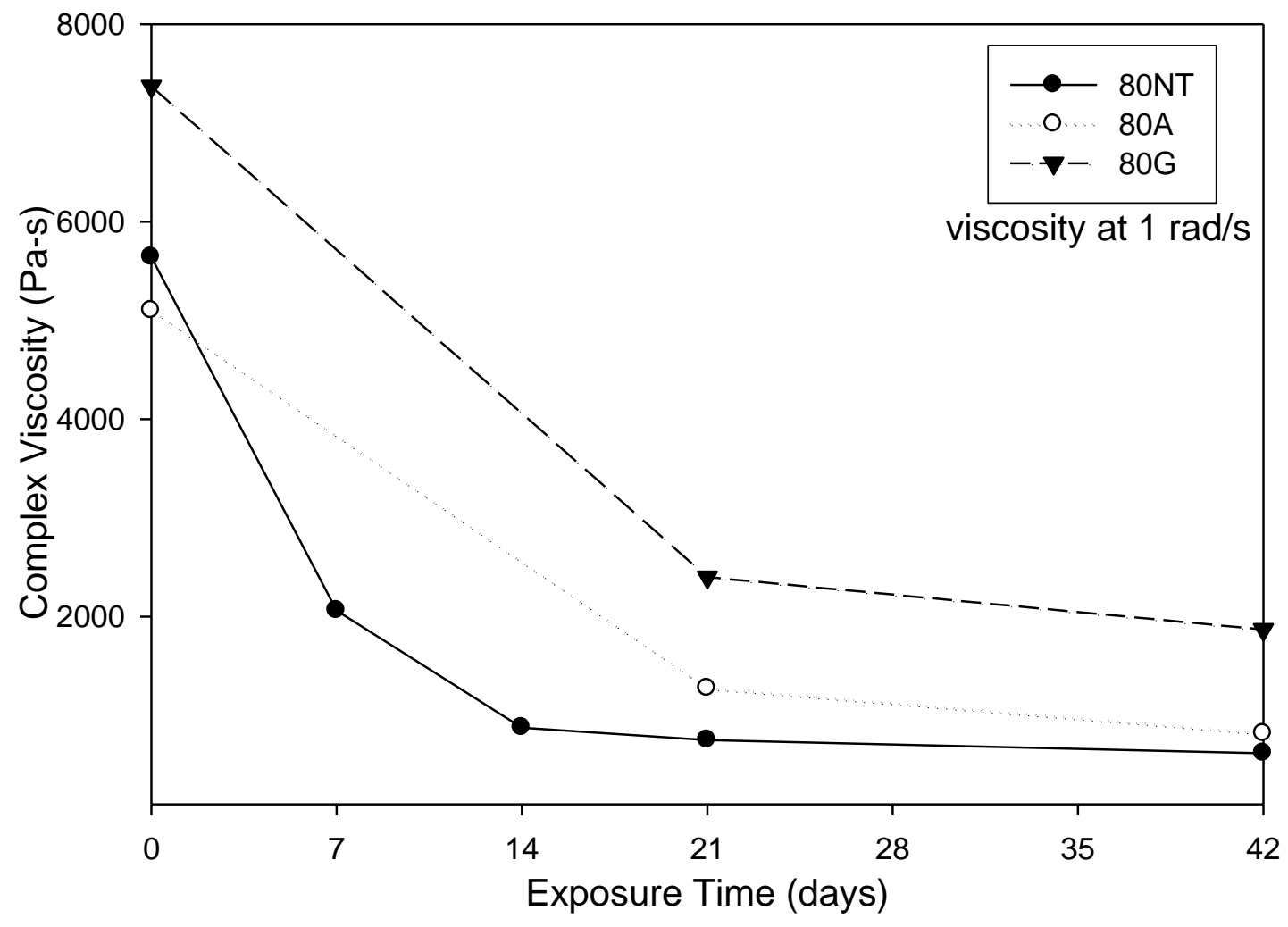

Figure 4.45: Comparison of viscosity loss between 79-80\% PLA blends with varying treatments.

Here, the untreated panel lost about $85 \%$ viscosity compared to roughly $75 \%$ for both the annealed and graphene-containing materials. This shows that the treatments have a tangible effect but are definitely insufficient protection against solvent degradation. Therefore, the best technique is to continue to increase the amount of polycarbonate present. Figures 4.46-49 show the complex viscosity as above for the remaining compositions with increasing PC content: 


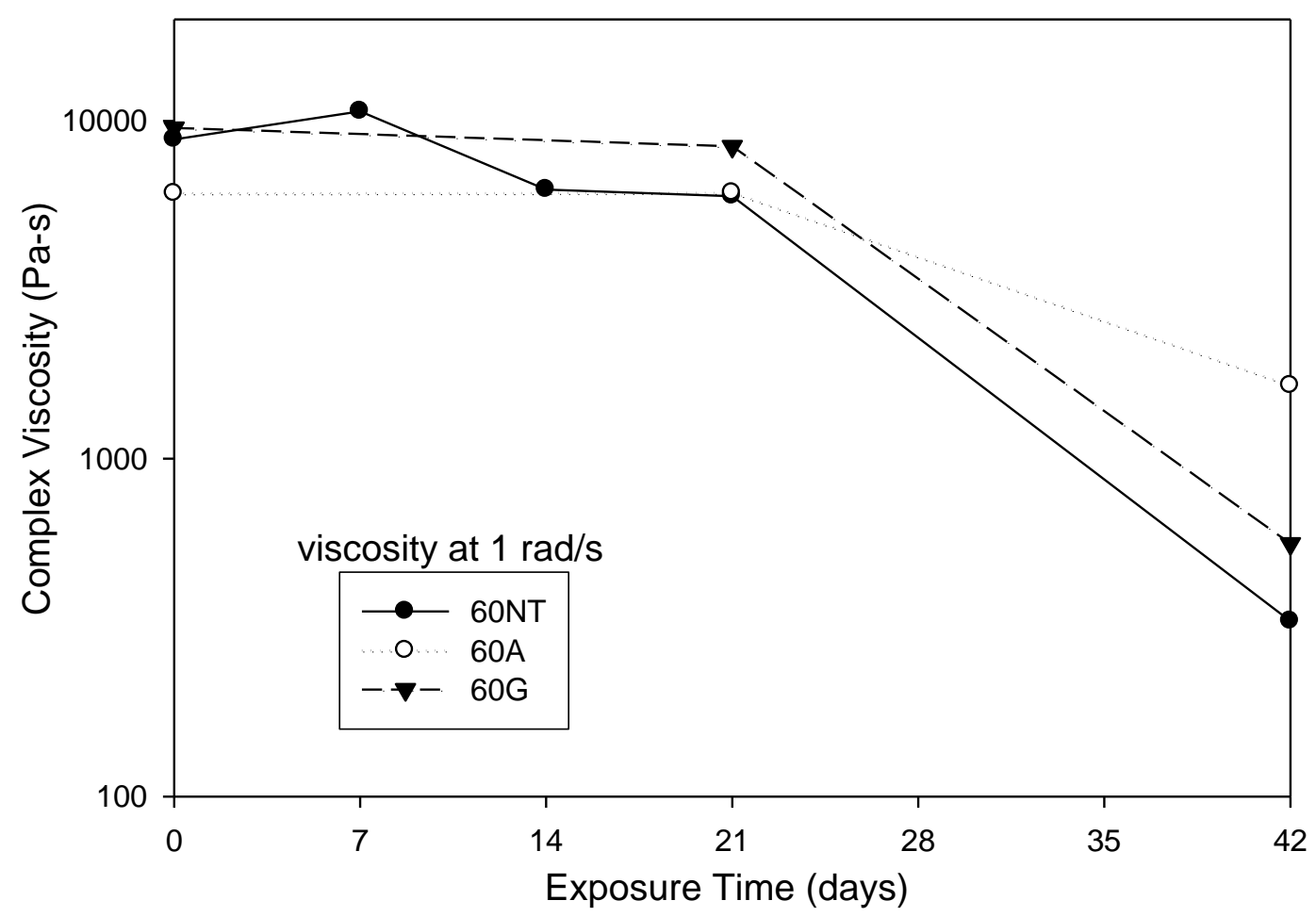

Figure 4.46: Comparison of viscosity loss between 59-60\% PLA blends with varying treatments.

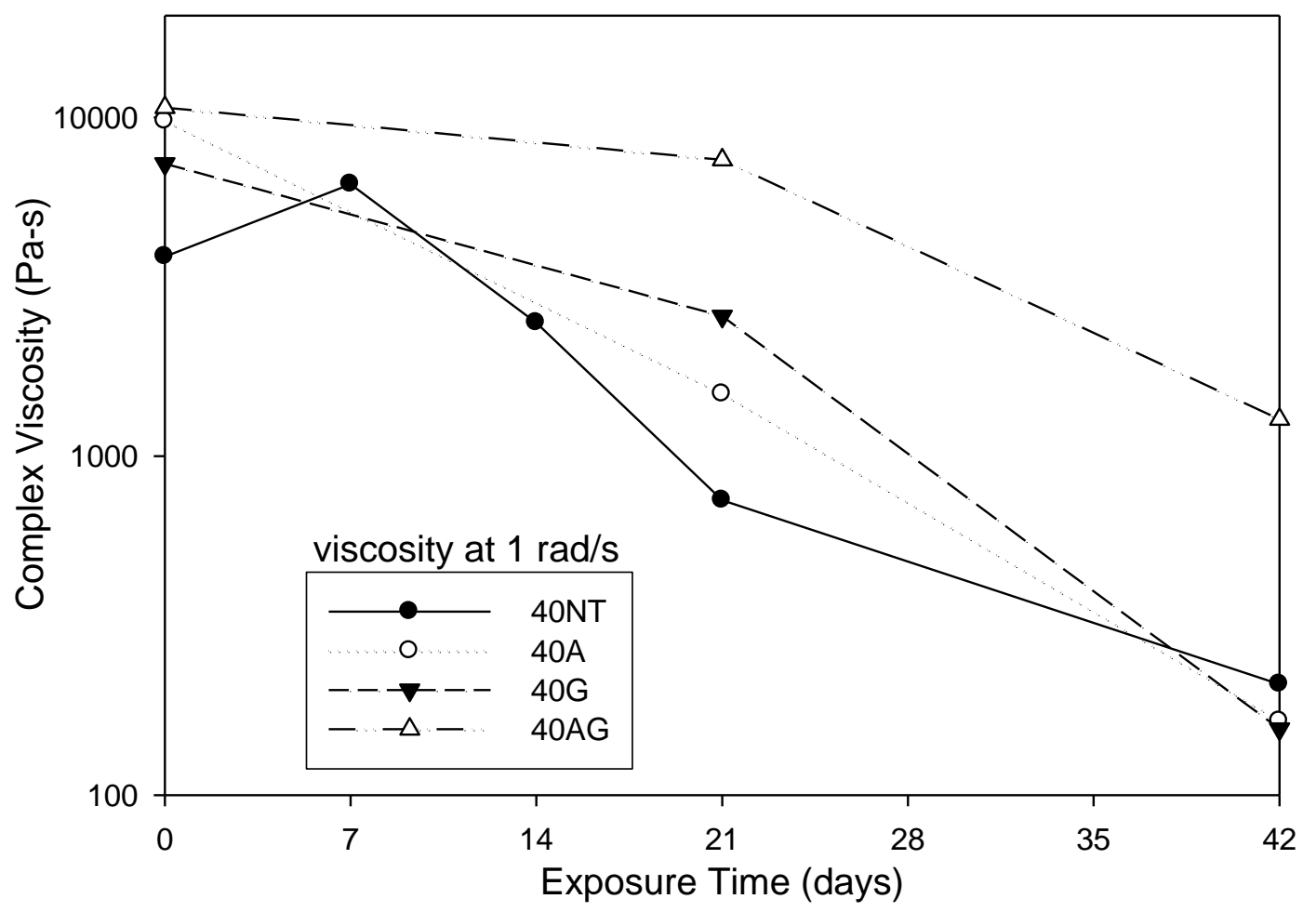

Figure 4.47: Comparison of viscosity loss between 39-40\% PLA blends with varying treatments. 


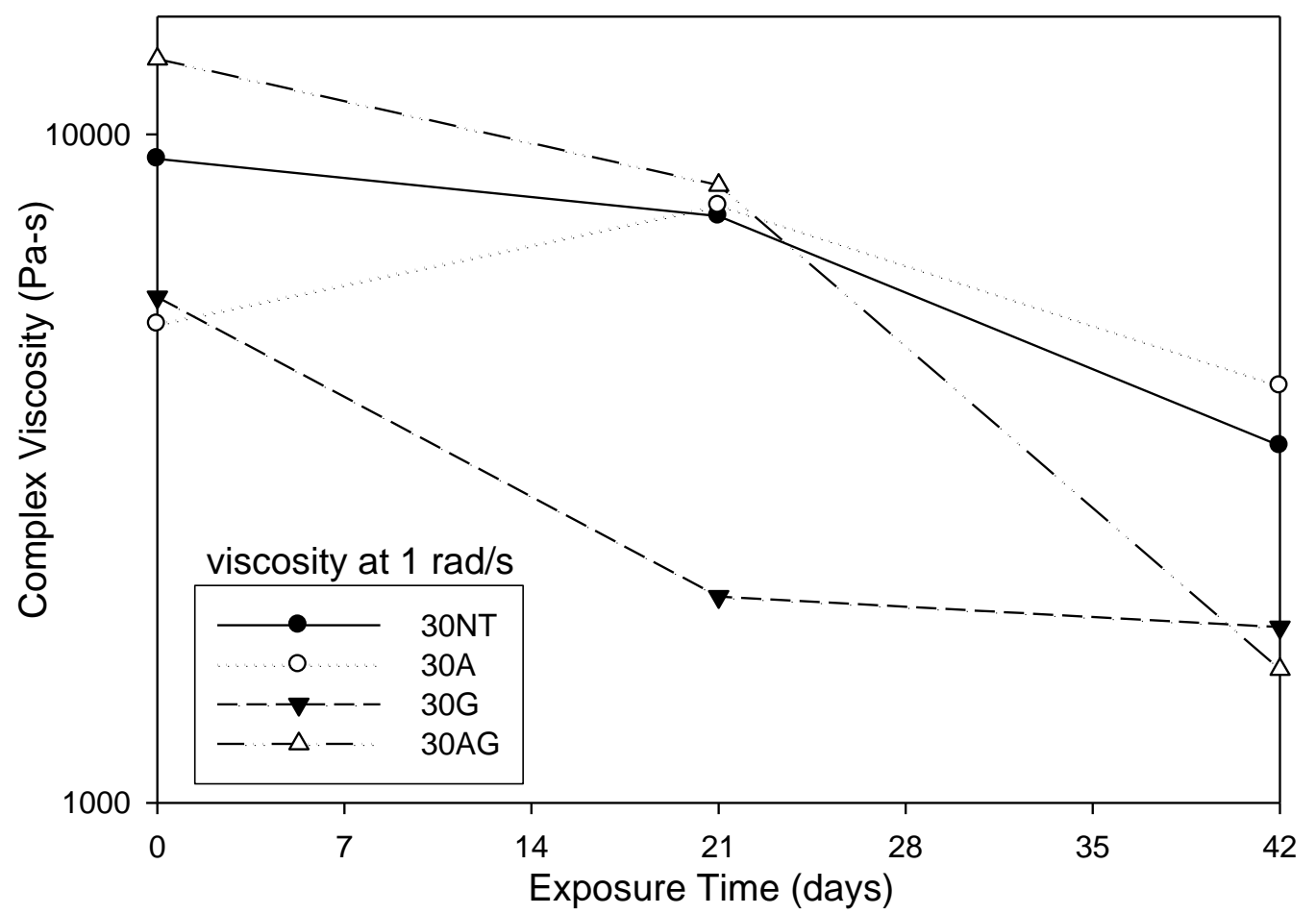

Figure 4.48: Comparison of viscosity loss between 29-30\% PLA blends with varying treatments.

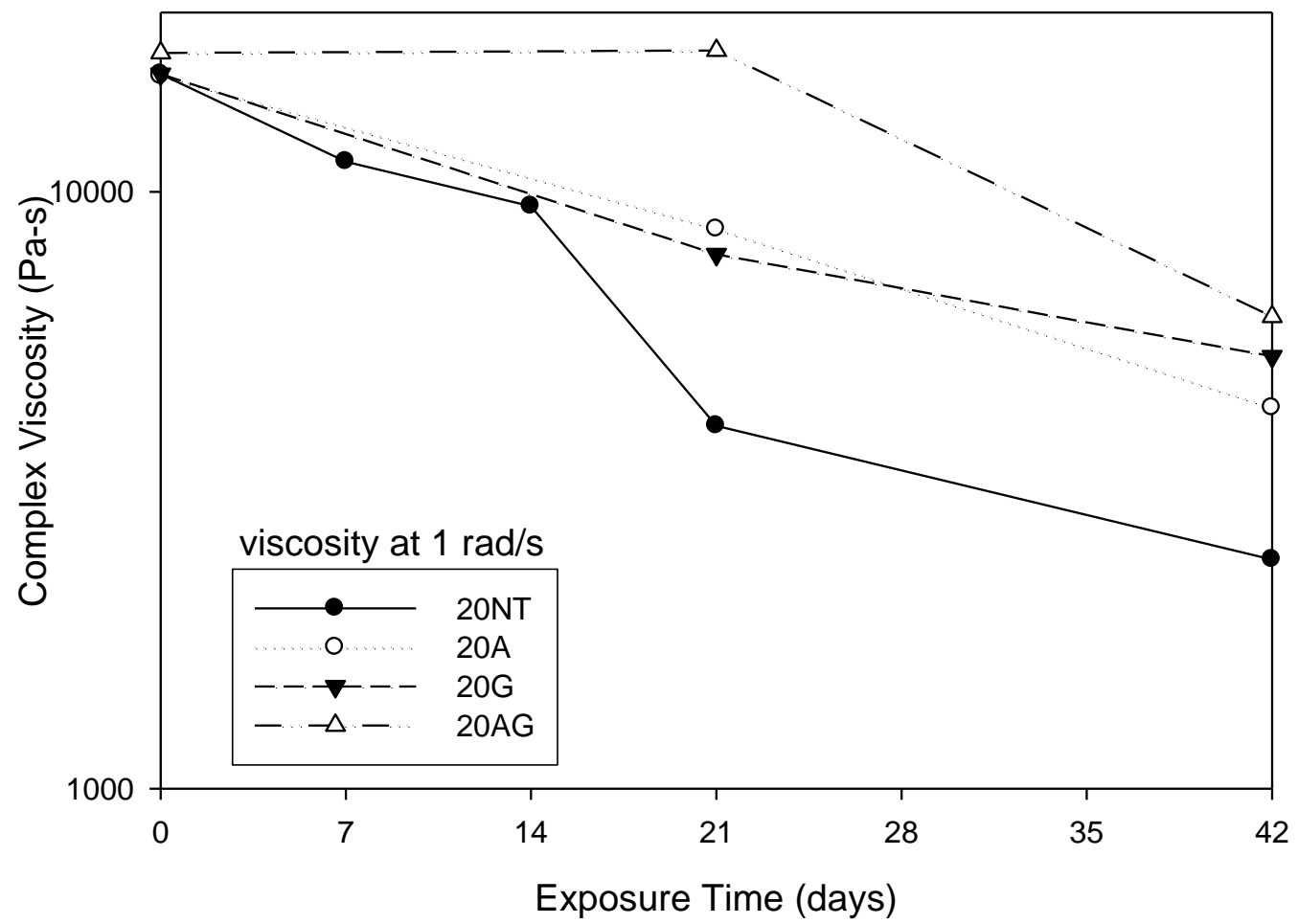

Figure 4.49: Comparison of viscosity loss between 19-20\% PLA blends with varying treatments. 


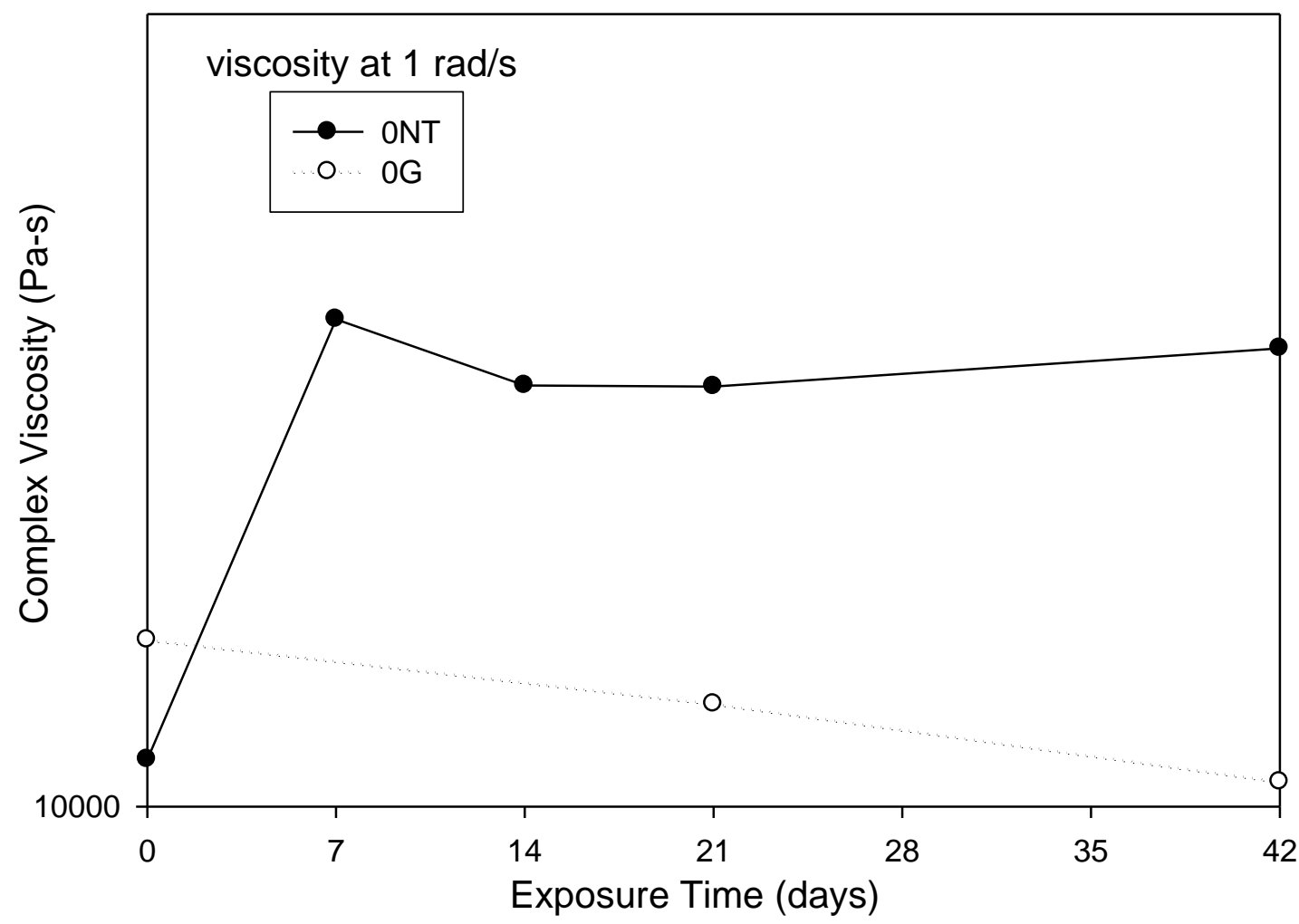

Figure 4.50 Comparison of viscosity loss between 0\% PLA blends with varying treatments.

The omitted total viscosity curves may be found in Appendix A. As the PC content increases, there is a demonstrably better retention of viscosity for the materials. The percentage viscosity loss for each material and treatment is listed here in Table 4.5, including the values for the PLAonly samples previously described:

Table 4.5: Viscosity loss at $1 \mathrm{rad} / \mathrm{s}$ after 42 days aging for the PC/PLA and PC/PLA/graphene composites.

\begin{tabular}{|r|c|c|c|c|}
\hline PLA content (\%) & no treatment & annealed & graphene & annealed/graphene \\
\hline $98-100$ & -99.9 & -99.4 & -99.5 & N/A \\
\hline $79-80$ & -86.7 & -75.0 & -74.6 & N/A \\
\hline $59-60$ & -94.0 & -73.0 & -94.1 & N/A \\
\hline $39-40$ & -94.5 & -98.3 & -97.9 & -88.0 \\
\hline $29-30$ & -62.8 & -22.4 & -67.9 & -87.8 \\
\hline $19-20$ & -84.6 & -72.2 & -66.4 & -63.8 \\
\hline 0 & +60.8 & N/A & -15.1 & N/A \\
\hline
\end{tabular}


From these data it becomes very clear that any material that is PLA-rich is unsuitable for any durable application and that about 30\% PLA content is the maximum desirable loading. The increase in viscosity for sample ONT is unusual but is supported by several replications. Depending on the treatment, one can minimize viscosity loss to about $15-30 \%$ at compositions containing $30 \%$ PLA or less. Interestingly, these data suggest that materials that are both annealed and that contain graphene do not have an improved resistance. The next step is to again observe the behavior of the PC/PLA and PC/PLA/graphene composites after aging in the environmental chamber.

\subsubsection{Hydrolytic Degradation of PC/PLA and PLA/Graphene Composites.}

\subsubsection{Effect of Hydrolytic Degradation on Mechanical Properties.}

The initial flexural properties of the PC/PLA blends and composites are given here in Figures 4.51-53:

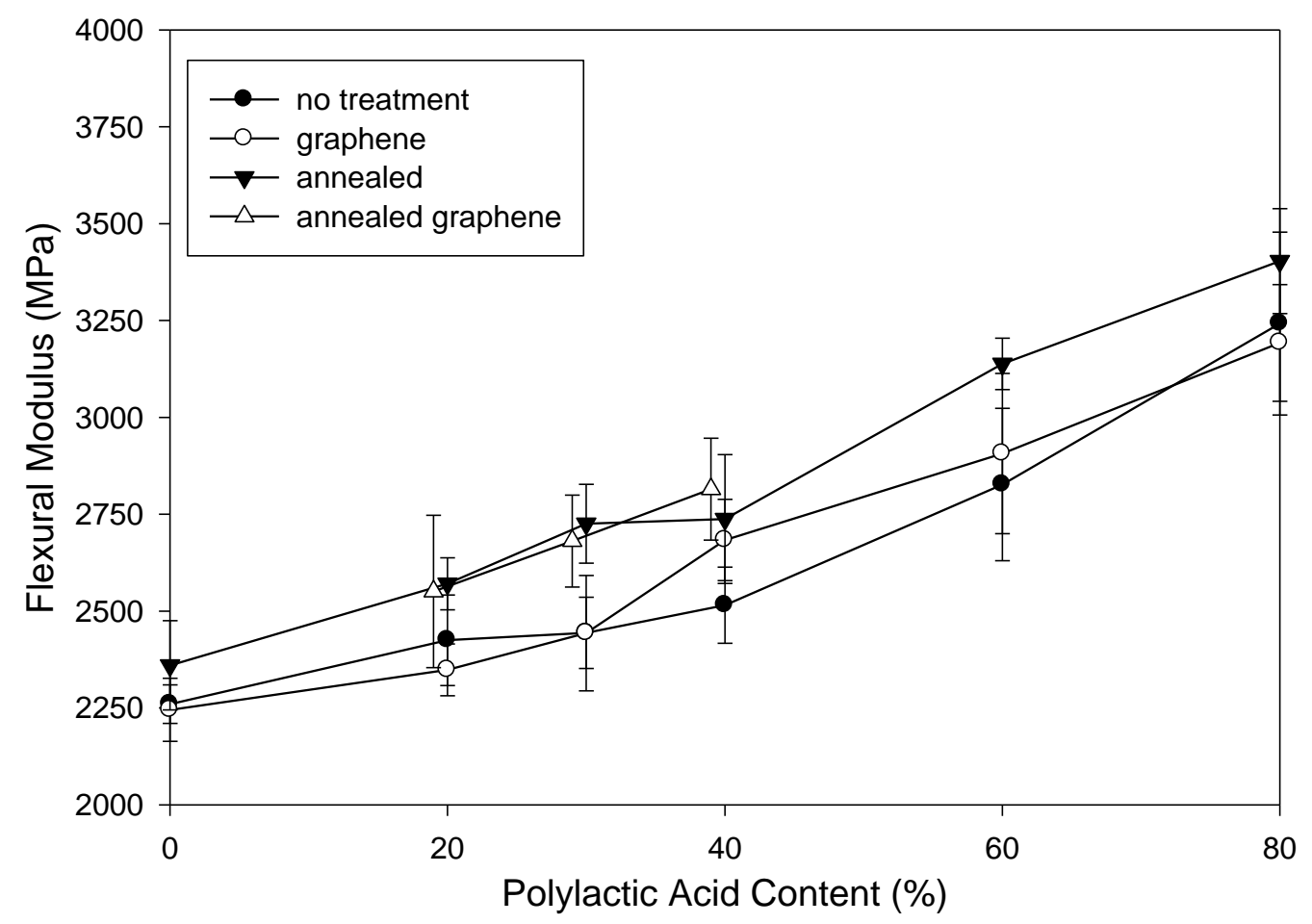

Figure 4.51: Initial flexural modulus of the PC/PLA and PC/PLA/graphene materials with various treatments. 


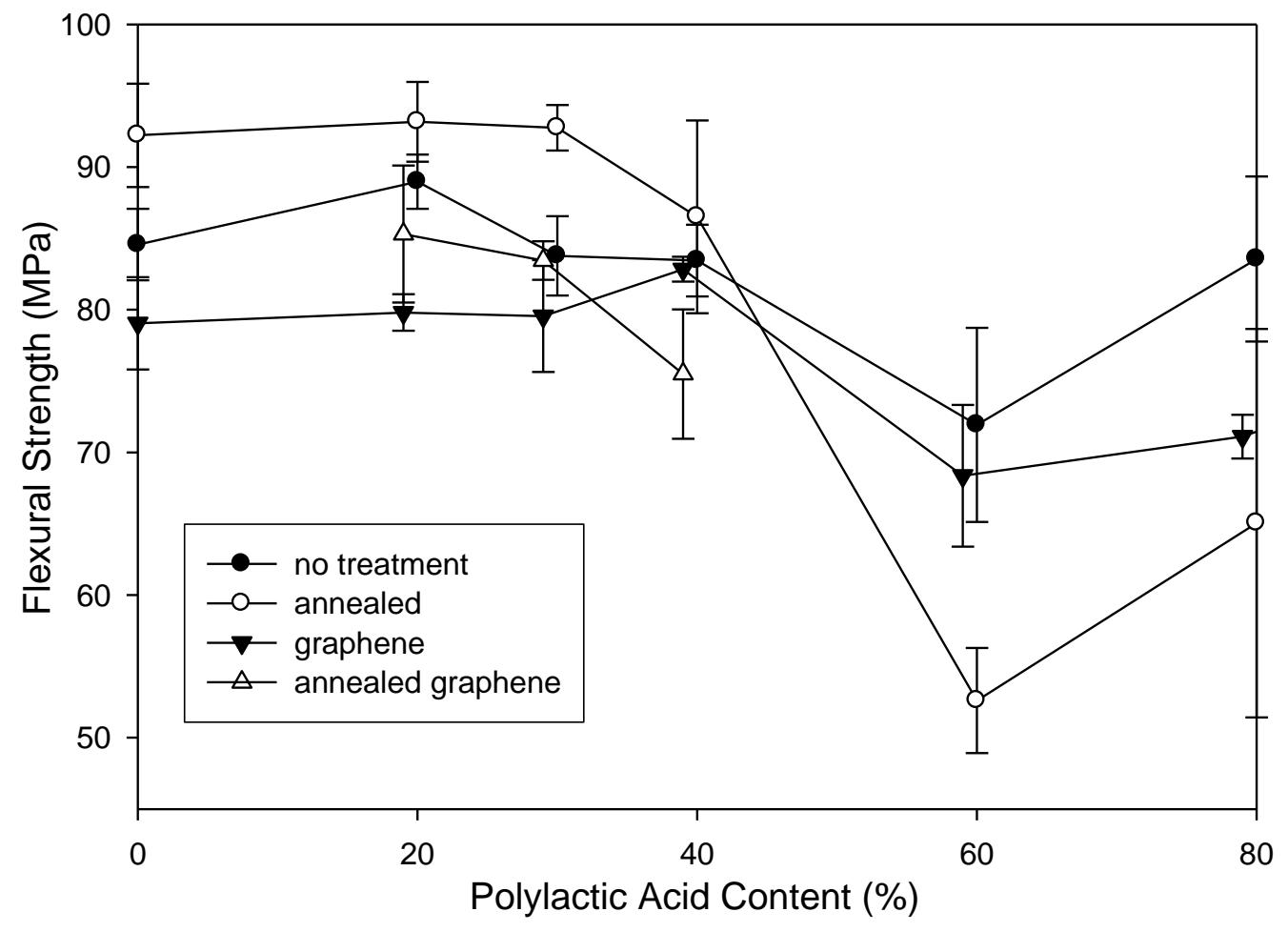

Figure 4.52: Initial flexural strength of the PC/PLA and PC/PLA/graphene materials with various treatments.

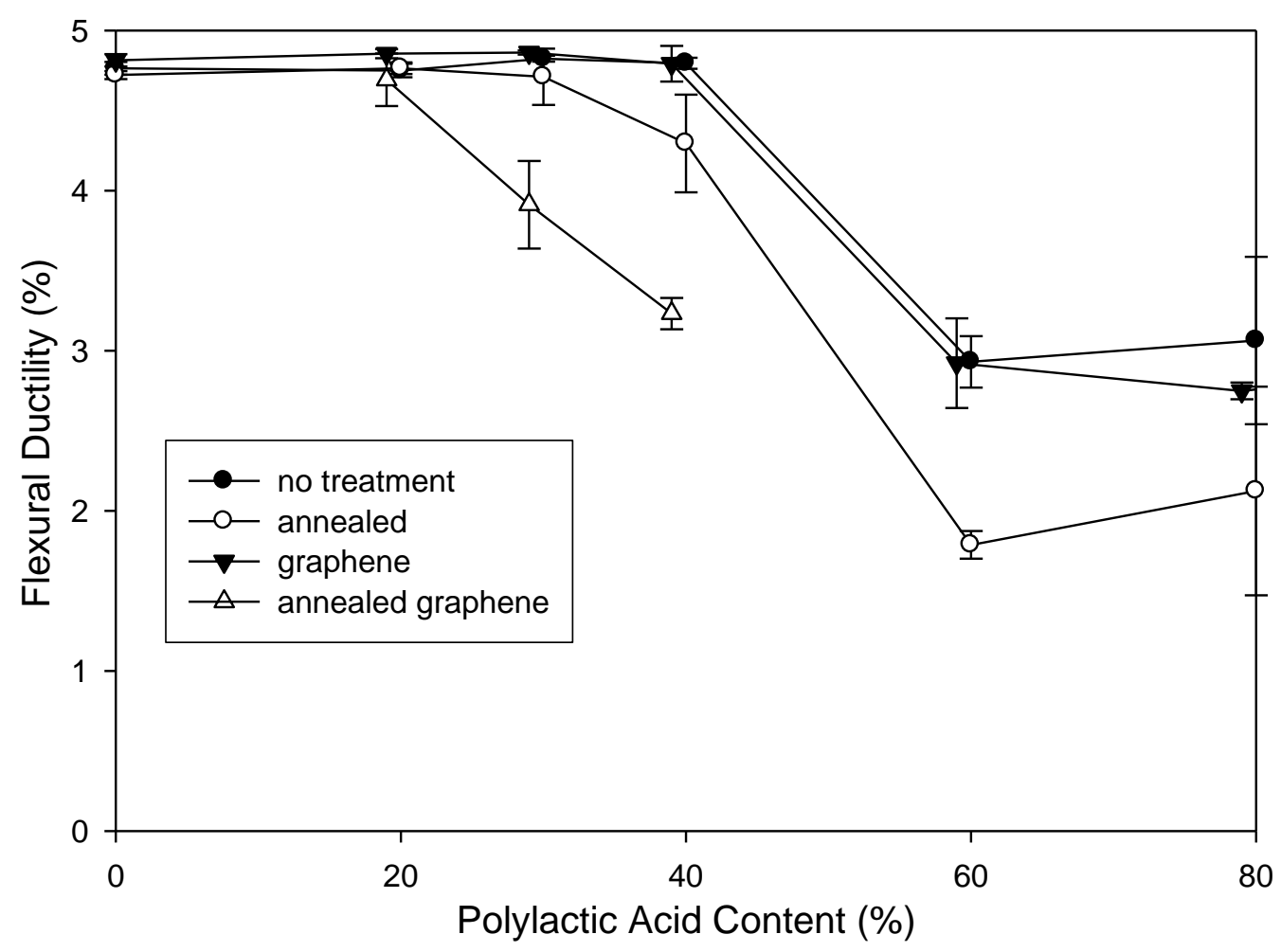

Figure 4.53: Initial flexural ductility of the PC/PLA and PC/PLA/graphene materials with various treatments. 
Figure 4.51 shows an expected result, but not for the strength and ductility; there may be a composition-based antagonism occurring in some formulations. Materials that are PLA-rich with any treatment are brittle and have low flexural strength as a result, but as the amount of highly ductile PC increases the materials become more ductile; we can also see that PLA has a higher modulus than PC. Annealing the PLA-rich samples improves the modulus and strength slightly as expected, but these materials are very brittle as well. As before, the change in flexural strength with aging is reported in Figure 4.54-57:

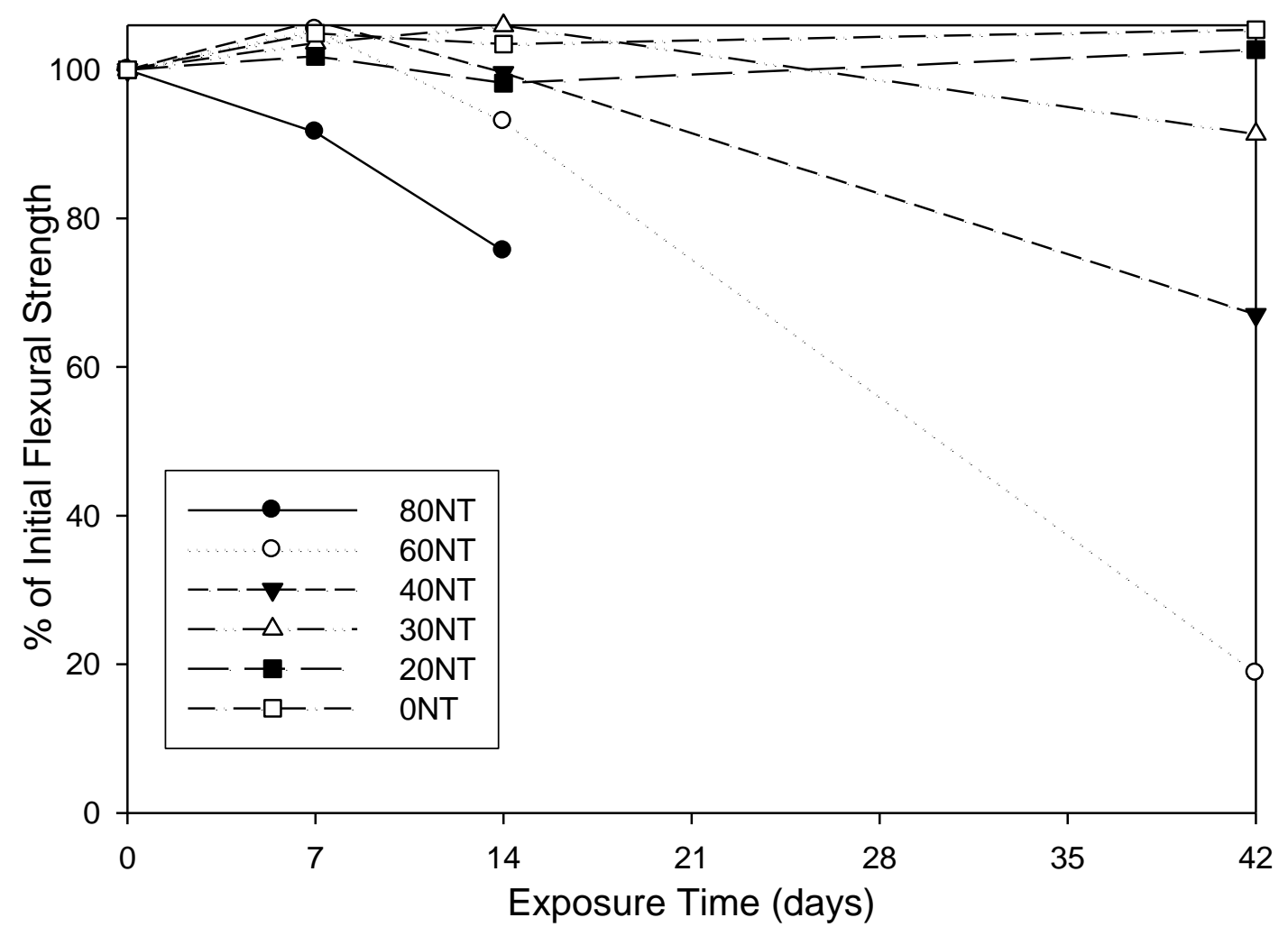

Figure 4.54: Flexural strength loss of untreated PC/PLA bars after hydrolysis. 


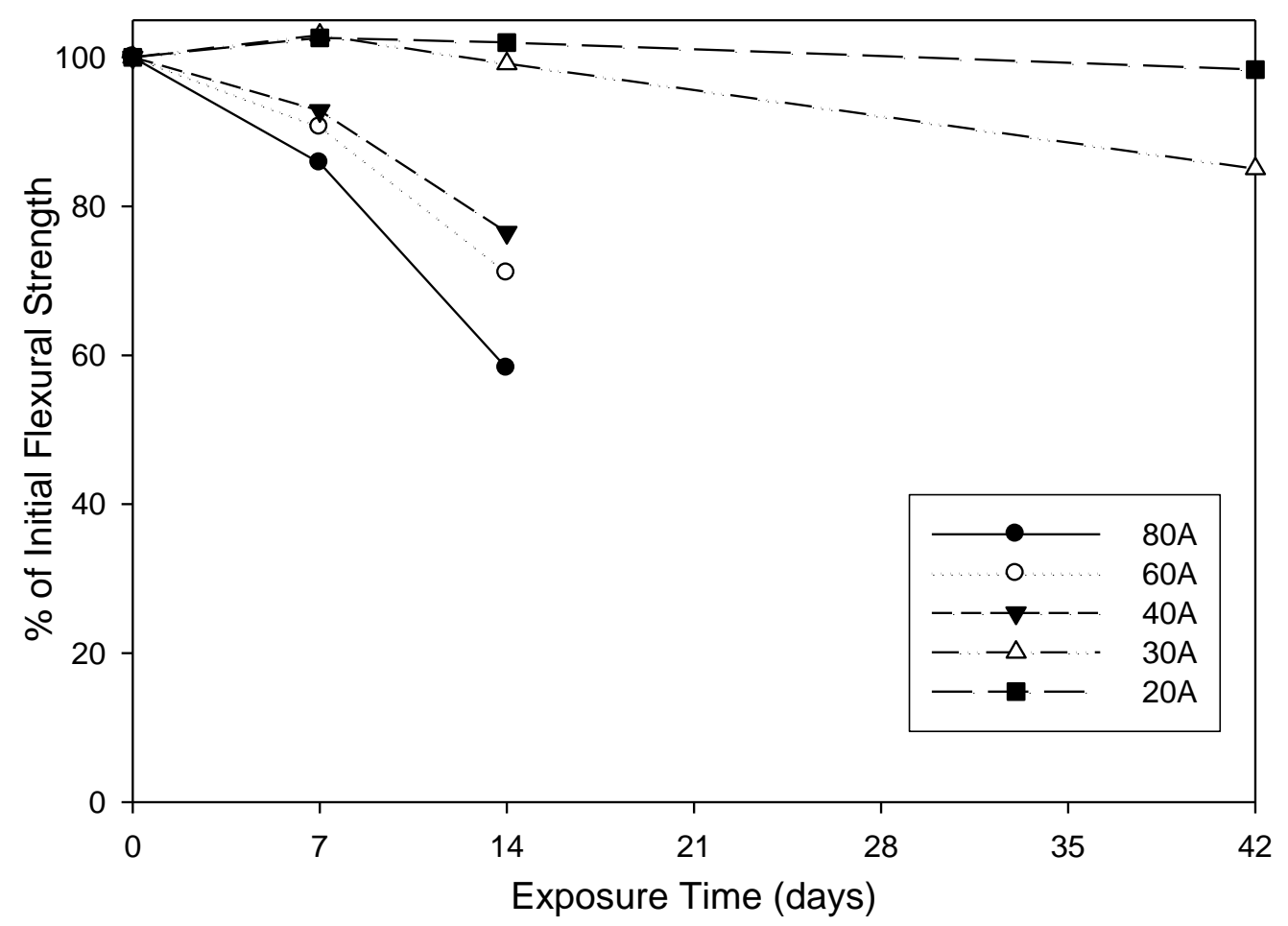

Figure 4.55: Flexural strength loss of annealed PC/PLA bars after hydrolysis.

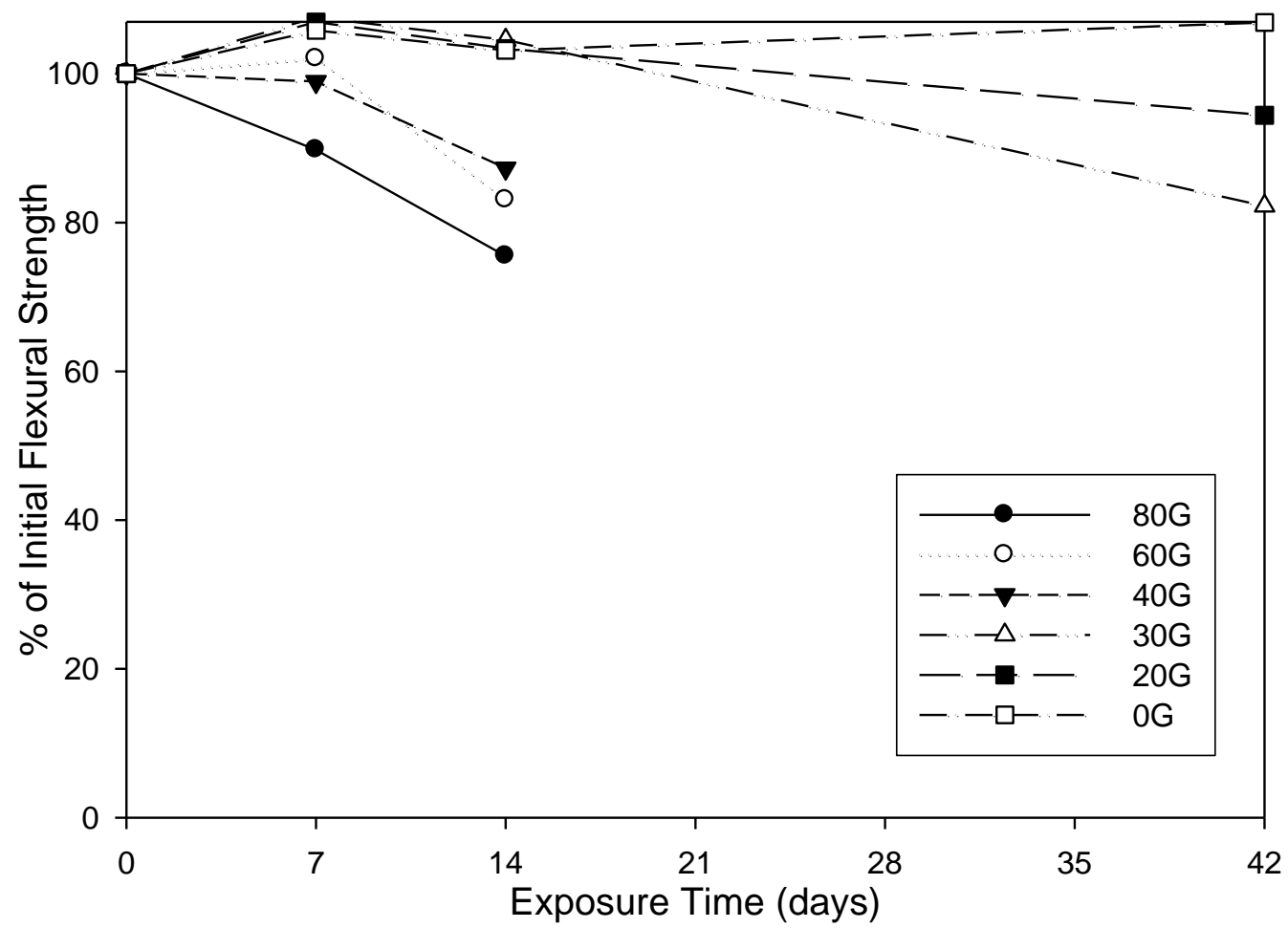

Figure 4.56: Flexural strength loss of PC/PLA/graphene bars after hydrolysis. 


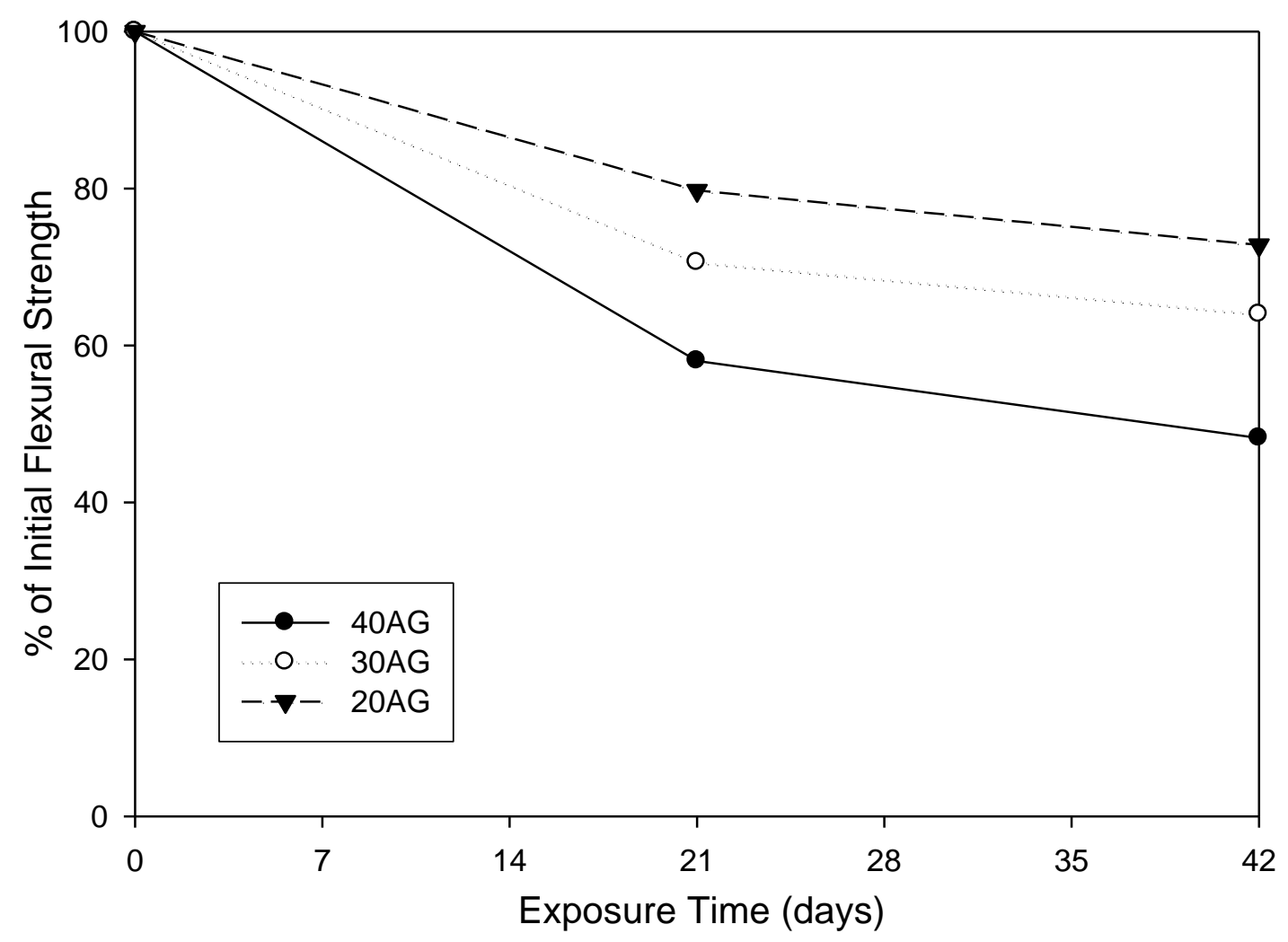

Figure 4.57: Flexural strength loss of annealed PC/PLA/graphene bars after hydrolysis.

Samples without treatment that are PLA-rich lose flexural strength and ductility very rapidly and are again shown to be too dimensionally unstable for use in durable applications. In terms of treatment options, both the annealing and graphene samples that are only 40\% PLA content (that is, still PC-rich!) are unsuitable for durable use as they are simply too brittle. Figure 4.56 shows that annealed/graphene samples with 30\% PLA content or less should be sturdy and sufficiently ductile for harsh environment applications.

The final examination of the PC/PLA and PC/PLA/graphene materials will come in the form of hydrolysis experiments carried out on pellets, again to remove any effect of diffusion resistance and give the worst-case data.

\subsubsection{Effect of Hydrolytic Degradation on PC/PLA and PC/PLA/Graphene Composites.}

The absolute values of the melt flow indices after varying exposure to the hydrolysis environment are plotted in Figures 4.58-61: 


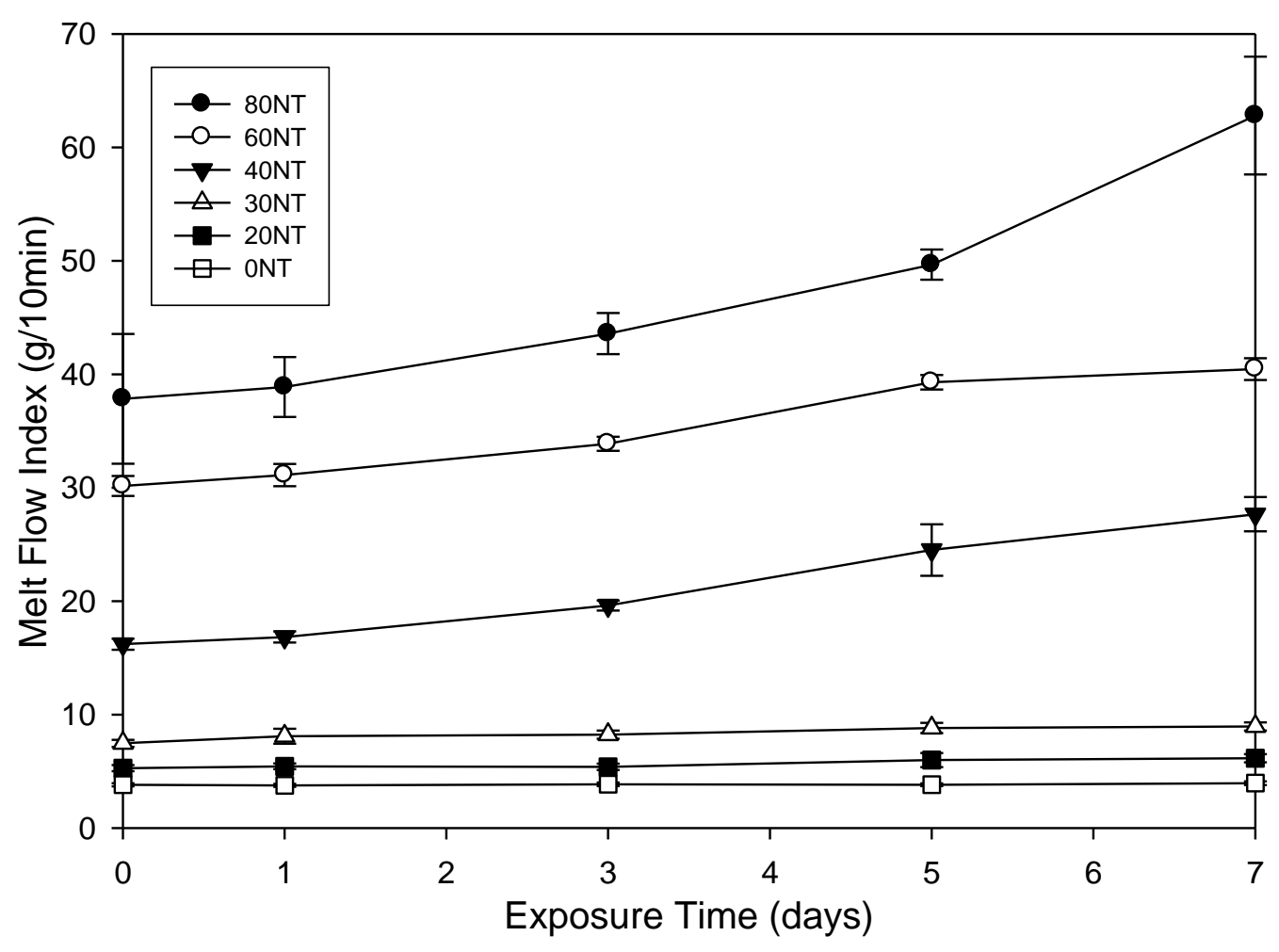

Figure 4.58: Increase in MFI of untreated PC/PLA pellets after hydrolysis.

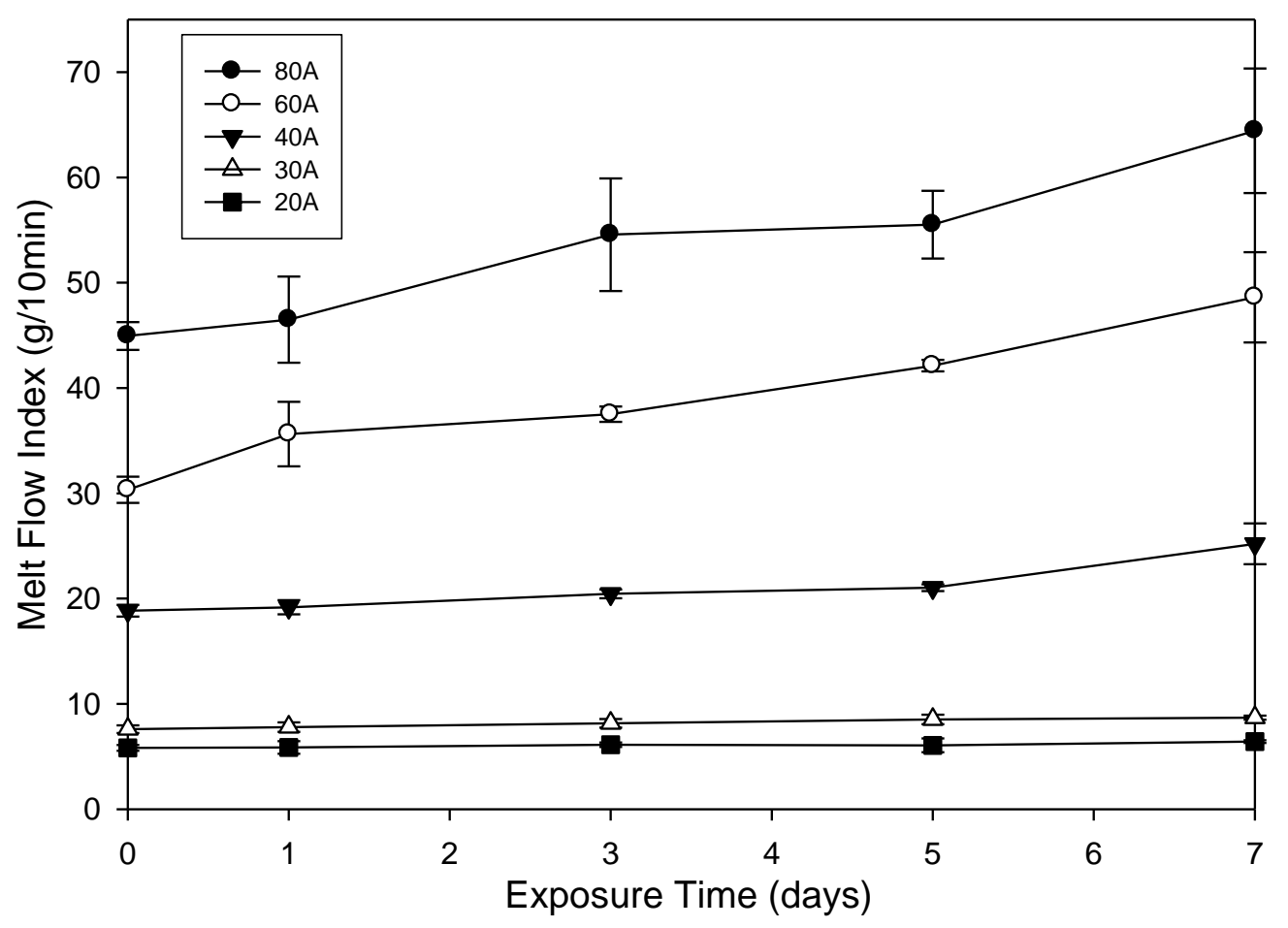

Figure 4.59: Increase in MFI of annealed PC/PLA pellets after hydrolysis. 


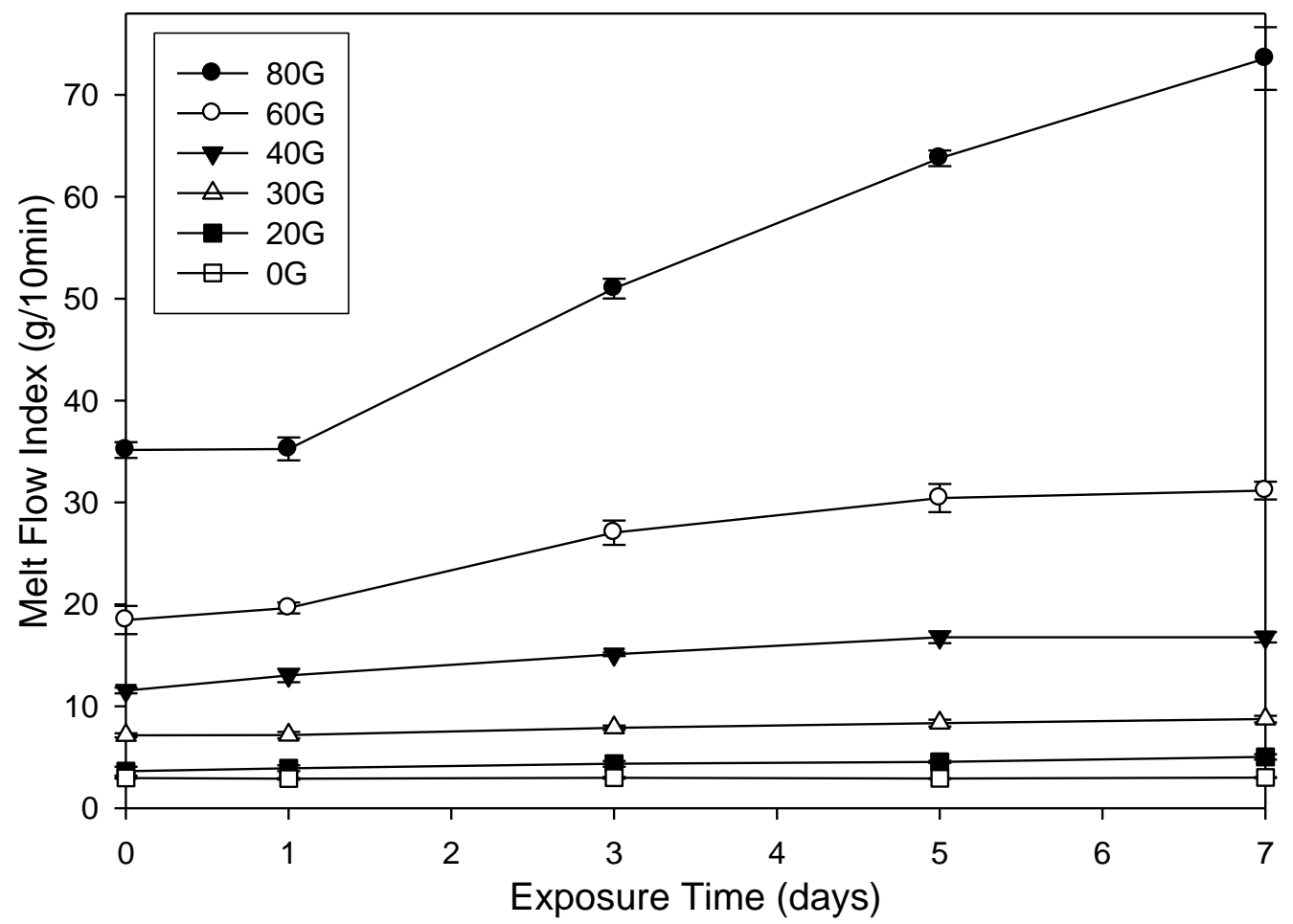

Figure 4.60: Increase in MFI of PC/PLA/graphene pellets after hydrolysis.

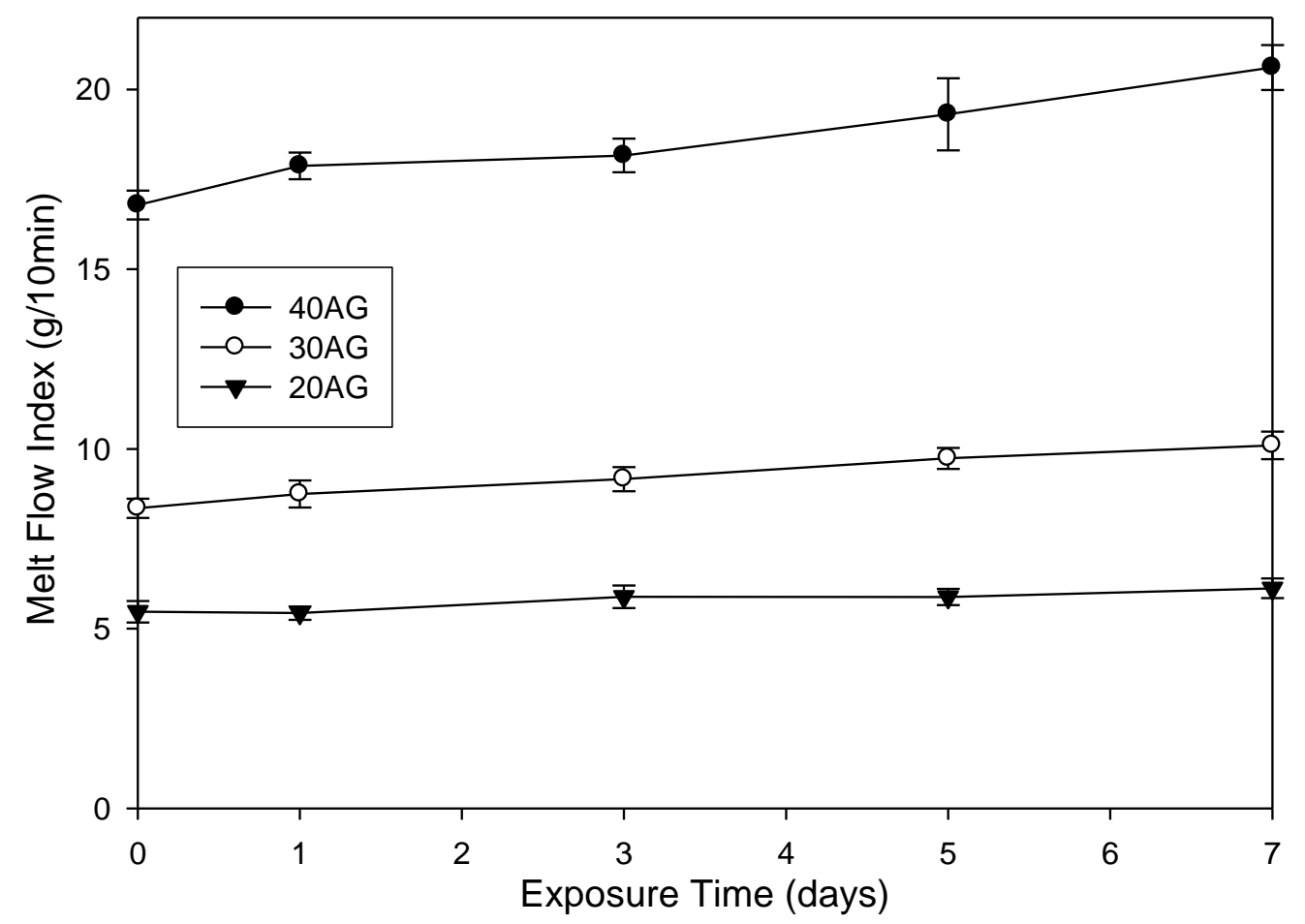

Figure 4.61: Increase in MFI of annealed PC/PLA/graphene pellets after hydrolysis. 
At first glance, the values for the different treatment options do not seem to indicate any discernable differences apart from the profound effect of composition. The annealed/graphene pellets also show this trend, where there is not much change in MFI with exposure but rather the difference lies in the formulation. We may now re-plot these figures and show the percent change of MFI to see if trends emerge in Figures 4.62-65:

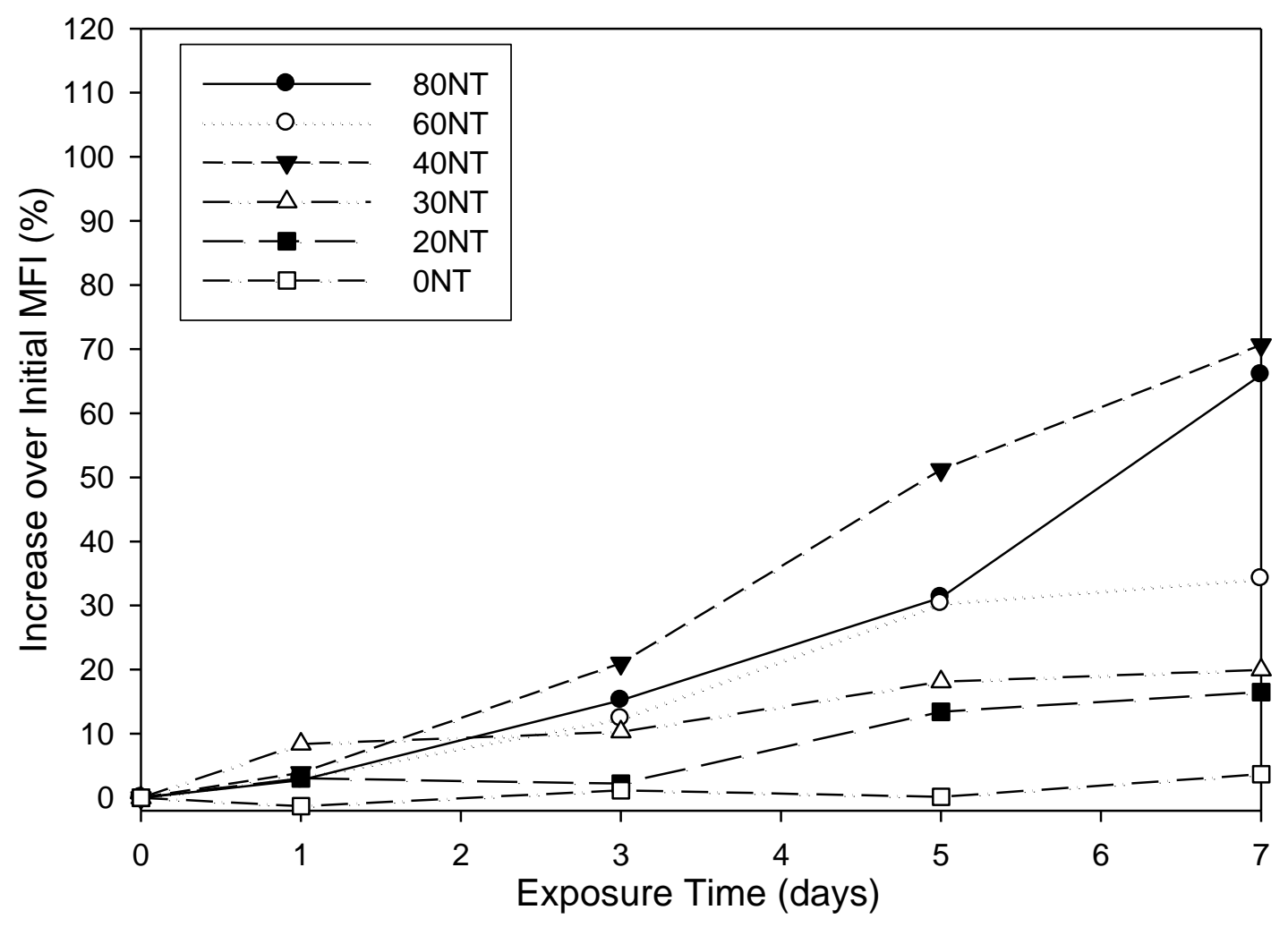

Figure 4.62: Normalized change in MFI for untreated PC/PLA pellets. 


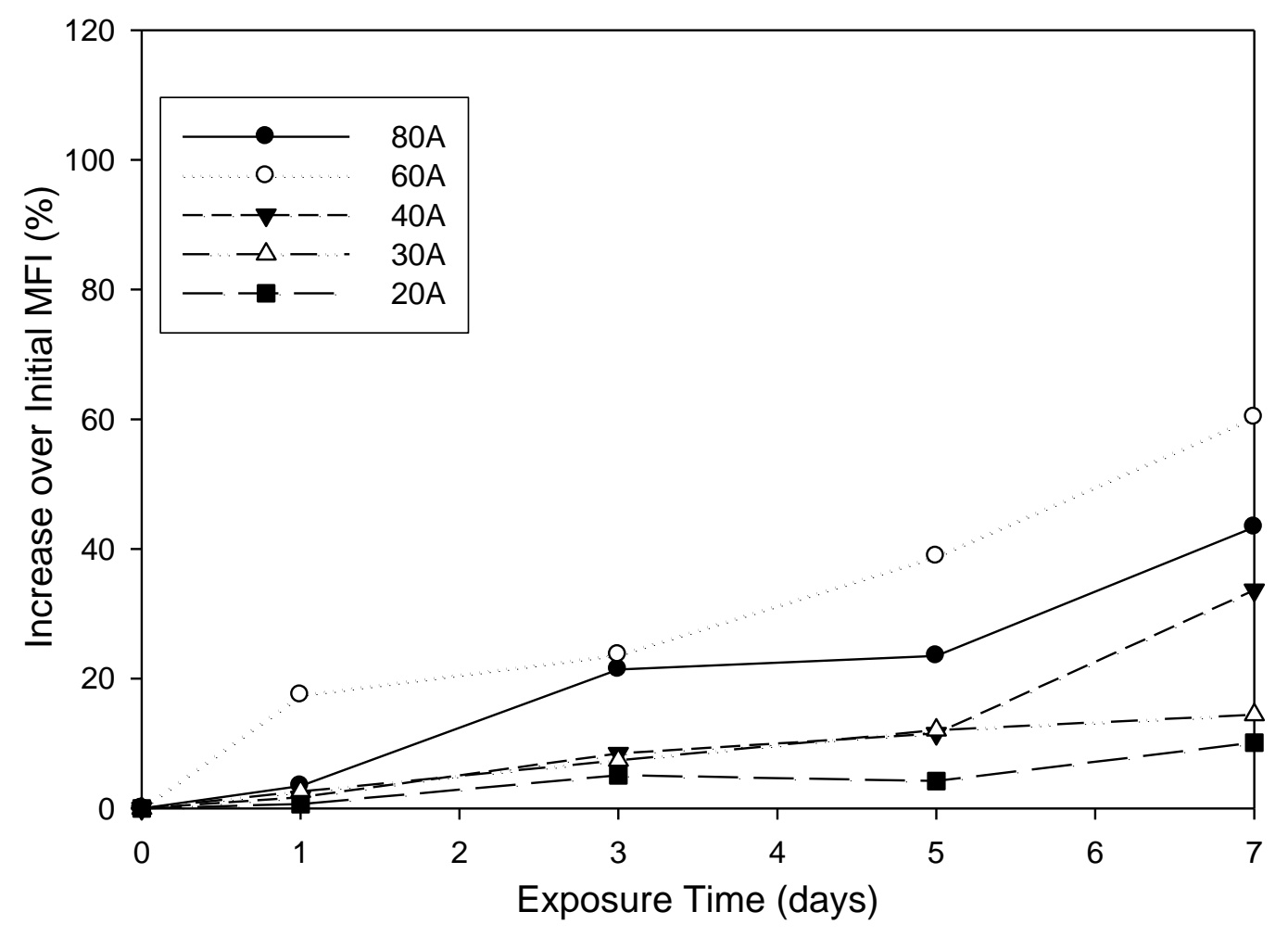

Figure 4.63: Normalized change in MFI for annealed PC/PLA pellets.

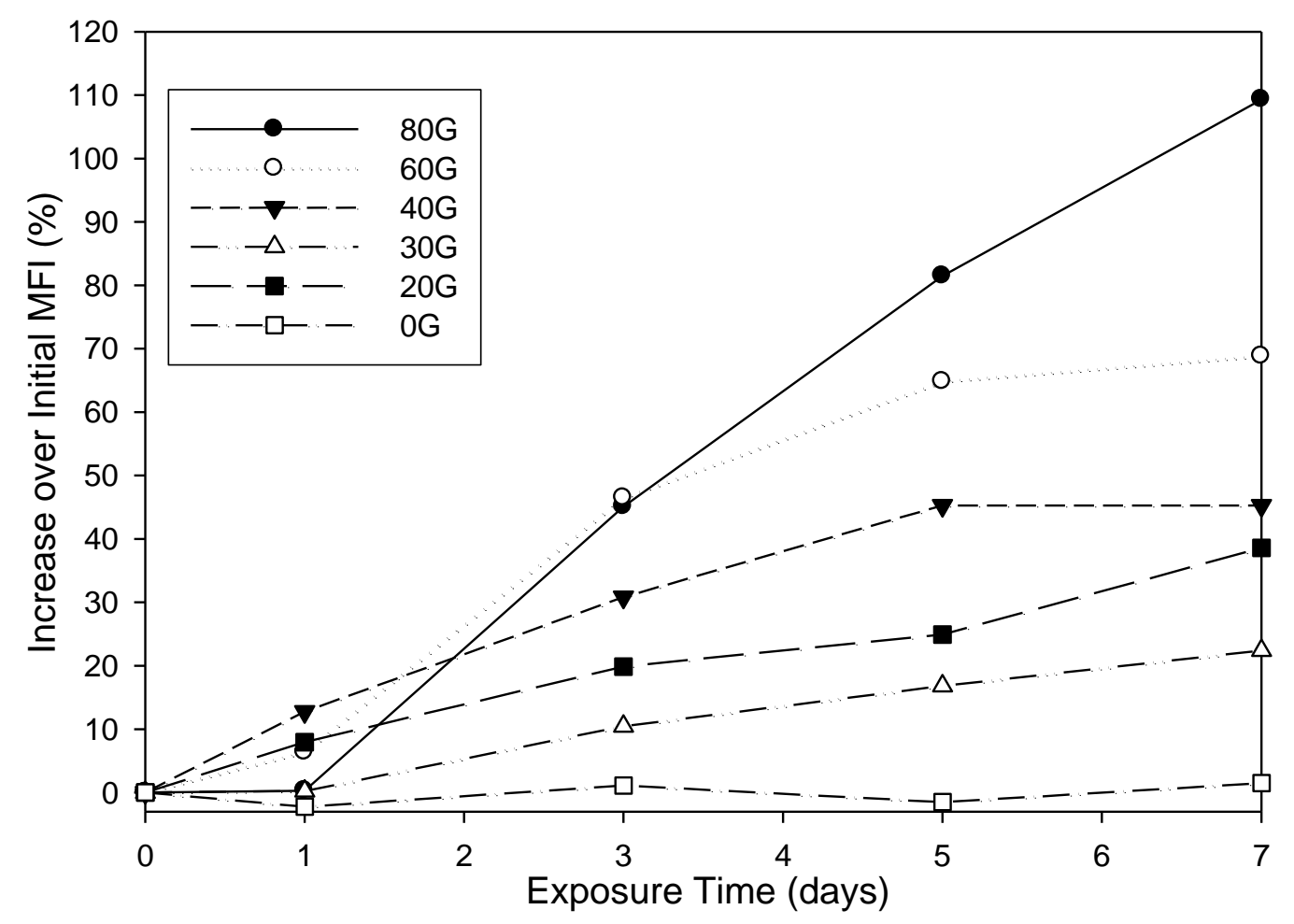

Figure 4.64: Normalized change in MFI for PC/PLA/graphene pellets. 


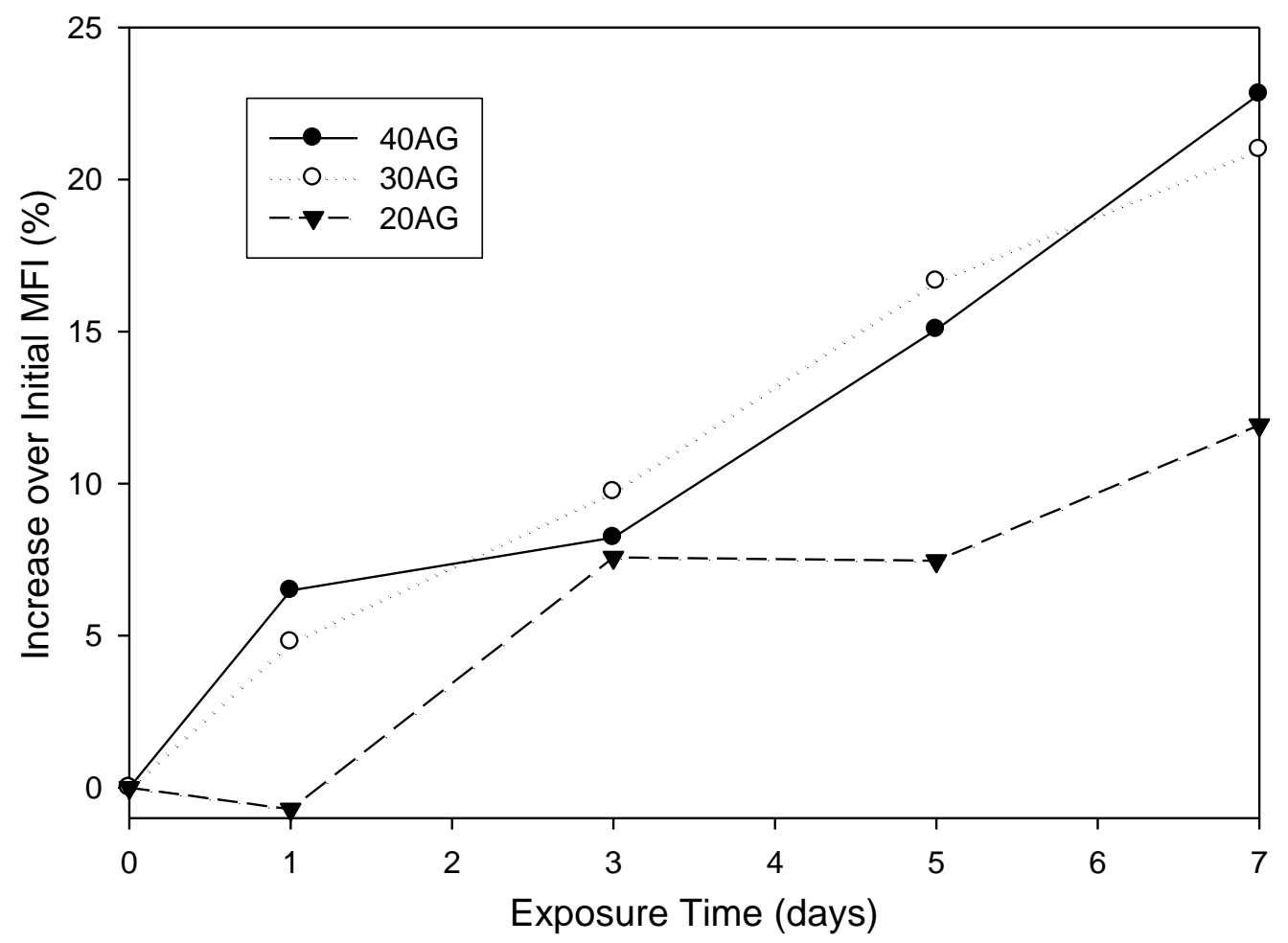

Figure 4.65: Normalized change in MFI for annealed PC/PLA/graphene pellets.

For the untreated, annealed and graphene-containing samples, any composition above 30\% PLA loading shows a significant increase in MFI after the 7 day exposure; the annealed/graphene pellets all give more satisfactory results. It is noted that both the untreated PC and the PC/graphene samples have essentially zero change in MFI, so it is likely in the other compositions that all of the increase comes from the degradation of the PLA phase. Therefore it is easy to generate an overall theme based on this set of results.

\subsection{Commentary on PC/PLA and PC/PLA/Graphene Durability.}

The same set of initial characterization and degradation experiments performed on PLA-only materials was reproduced using binary (PC/PLA) and ternary (PC/PLA/graphene) formulations to attempt to improve the very poor durability. It was shown that the presence of moderate to high polycarbonate loading does not significantly alter the crystallization of the PLA phase, but $60 \%$ or higher PC concentrations interfere with the kinetics. The effects of annealing, graphene addition and the combination of both on material durability were investigated; it was again shown that both treatments create a transient diffusion barrier which can slightly increase the usable lifetime of materials, but the complete encapsulation of PLA by PC is shown to have a 
tremendous effect on durability, as the PC is much more hydrolytically stable. Samples with $30 \%$ PLA phase or less are much more stable than any PLA-rich composition with any treatment option.

The last processing objective to investigate is to alter the interfacial morphology of a fully encapsulated blend. If the droplet structure can be affected by different mixing conditions, then it may be possible to create morphologies that minimize interfacial surface area which could create another diffusion barrier and increase the service life cycle of the PC/PLA blends.

\subsection{Fully Encapsulated 70/30 PC/PLA Blends.}

This set of fully encapsulated blends was examined to ascertain if any changes in processing conditions could affect the morphology in a significant way that could improve hydrolytic stability. The following table describes the internal mixing conditions used to fabricate these compositions:

Table 4.6: Mixing conditions for encapsulated 70/30 PC/PLA blends.

\begin{tabular}{|l|c|}
\hline Sample & Mixing Conditions \\
\hline $30 B$ & $240^{\circ} \mathrm{C}-40 \mathrm{rpm}-5$ minutes \\
\hline $30 C$ & $240^{\circ} \mathrm{C}-80 \mathrm{rpm}-5$ minutes \\
\hline $30 D$ & $240^{\circ} \mathrm{C}-40 \mathrm{rpm}-3$ minutes \\
\hline $30 E$ & $240^{\circ} \mathrm{C}-80 \mathrm{rpm}-15$ minutes \\
\hline
\end{tabular}

The extruded composition $30 N T$ was used as a reference material. The morphologies of these blends were collected in the same manner as all previous materials using the SEM and are shown in Figure 4.66: 

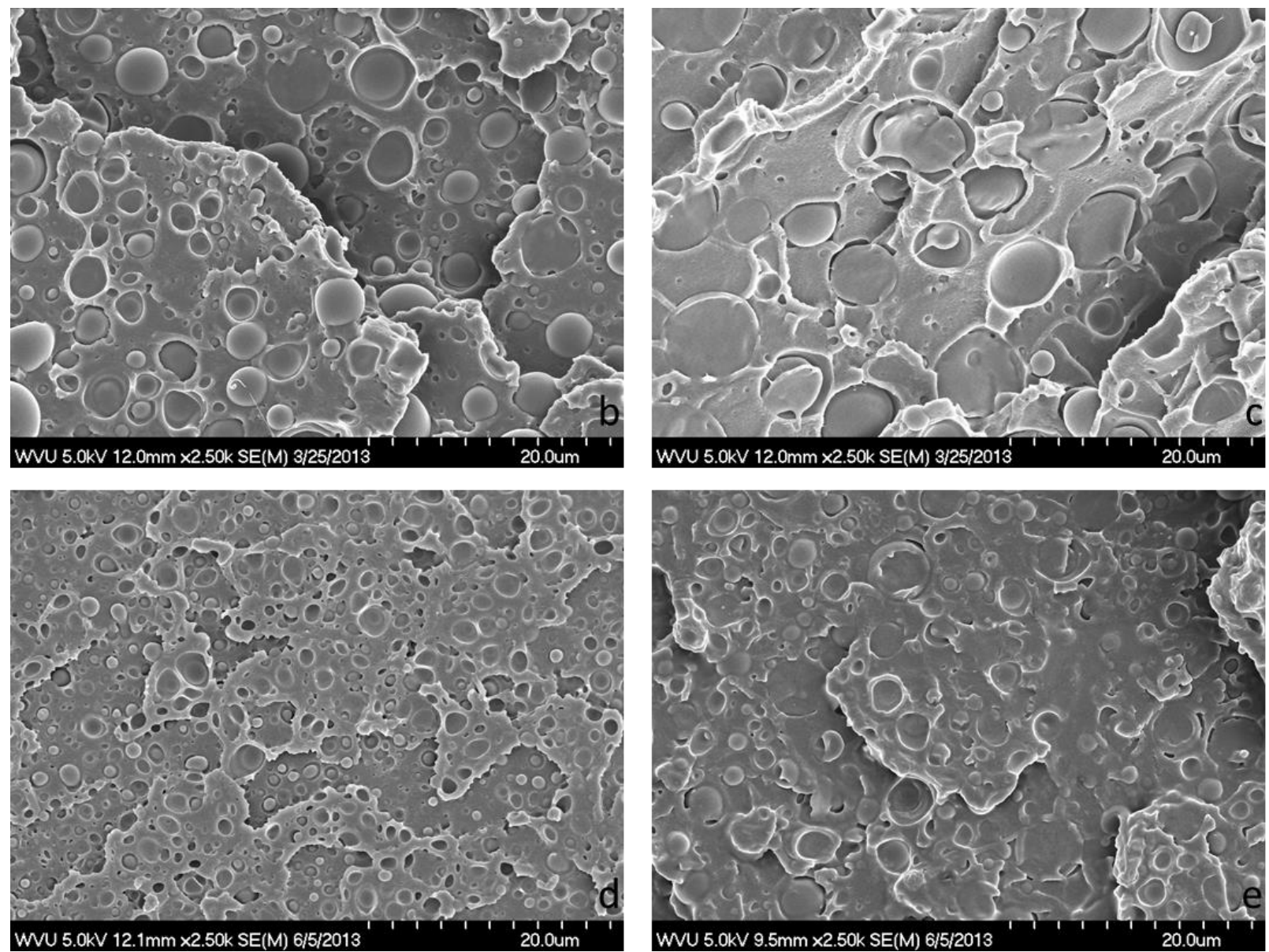

Figure 4.66: Images of internally mixed 70/30 PC/PLA blends 30B-E.

The small letter at the bottom right of each image denotes which blend is shown. Droplet sizes were calculated as before, and here sample $30 N T$ is included as well in Table 4.7:

Table 4.7: Particle sizes for the 70/30 PC/PLA blends.

\begin{tabular}{|l|r|r|}
\hline Sample & dB avg diam. $\mathbf{d}_{\mathbf{4 3}}(\boldsymbol{\mu m})$ & num. avg diam. $\mathbf{d}_{\mathbf{1 0}}(\boldsymbol{\mu m})$ \\
\hline $30 N T$ & 1.30 & 0.93 \\
\hline $30 B$ & 3.46 & 1.25 \\
\hline $30 C$ & 5.90 & 1.70 \\
\hline $30 D$ & 1.54 & 0.75 \\
\hline $30 E$ & 2.83 & 1.30 \\
\hline
\end{tabular}

Comparing these results to those from the previous blends, the sizes are similar to those of the 20 and 30\% PLA materials; it seems that the PC-rich blends provide morphologies with smaller dispersed phase droplets than those that are PLA-rich. Knowing the composition of these blends, 
one can show through a mass balance that in a highly theoretical sample of perfect monodisperse spheres, a morphology that contains only particles that are $d_{10}$ size will have an interfacial surface area that is $d_{43} / d_{10}$ times larger than a morphology that consists of spheres that are $d_{43}$ in size. This is sensible because for a given volume of dispersed phase particles, a large number of smaller droplets will have a larger surface area than a small number of large particles. For another highly stylized representation of these morphologies, if the $d_{43}$ and $d_{10}$ particle sizes for material 30NT are used as a benchmark, then the internally mixed samples would have the following normalized interfacial areas in Table 4.8:

Table 4.8: Theoretical interfacial surface areas of internally mixed 70/30 blends with respect to extruded blend.

\begin{tabular}{|l|r|r|}
\hline Sample & $\boldsymbol{d}_{\mathbf{4 3}}$ surface area relative to $\mathbf{3 0 N T}(\boldsymbol{\%})$ & $\boldsymbol{d}_{10}$ surface area relative to $\mathbf{3 0 N T}(\boldsymbol{\%})$ \\
\hline $30 N T$ & 100 & 100 \\
\hline $30 B$ & 37.5 & 74.4 \\
\hline $30 C$ & 22.0 & 54.7 \\
\hline $30 D$ & 84.4 & 124 \\
\hline $30 E$ & 45.9 & 71.5 \\
\hline
\end{tabular}

If there is an effect of morphology on the degradation resistance of these blends, one should logically expect that the internally mixed samples with the smaller particle sizes should be slightly less resistant than material $30 N T$ with the larger particles.

These samples were not analyzed for any crystalline domains as this was not the focus of the investigation; the only effect of interest was that of the particle size. Further, as we have shown in the previous sections, the influence of crystallinity is dramatically overshadowed by the effect of polycarbonate loading. We again examine the results of solvent and hydrolytic degradation for these materials.

\subsubsection{Solvent Degradation of Fully Encapsulated PC/PLA Blends.}

Compression molded panels of these blends were exposed to distilled water at $50^{\circ} \mathrm{C}$ for up to 42 days aging. None of the panels lost any appreciable mass during this time; because the PLA was encased by the much more stable PC phase this was not unexpected. The viscosity changes for the various morphologies are shown as the following Figures 4.67-71: 


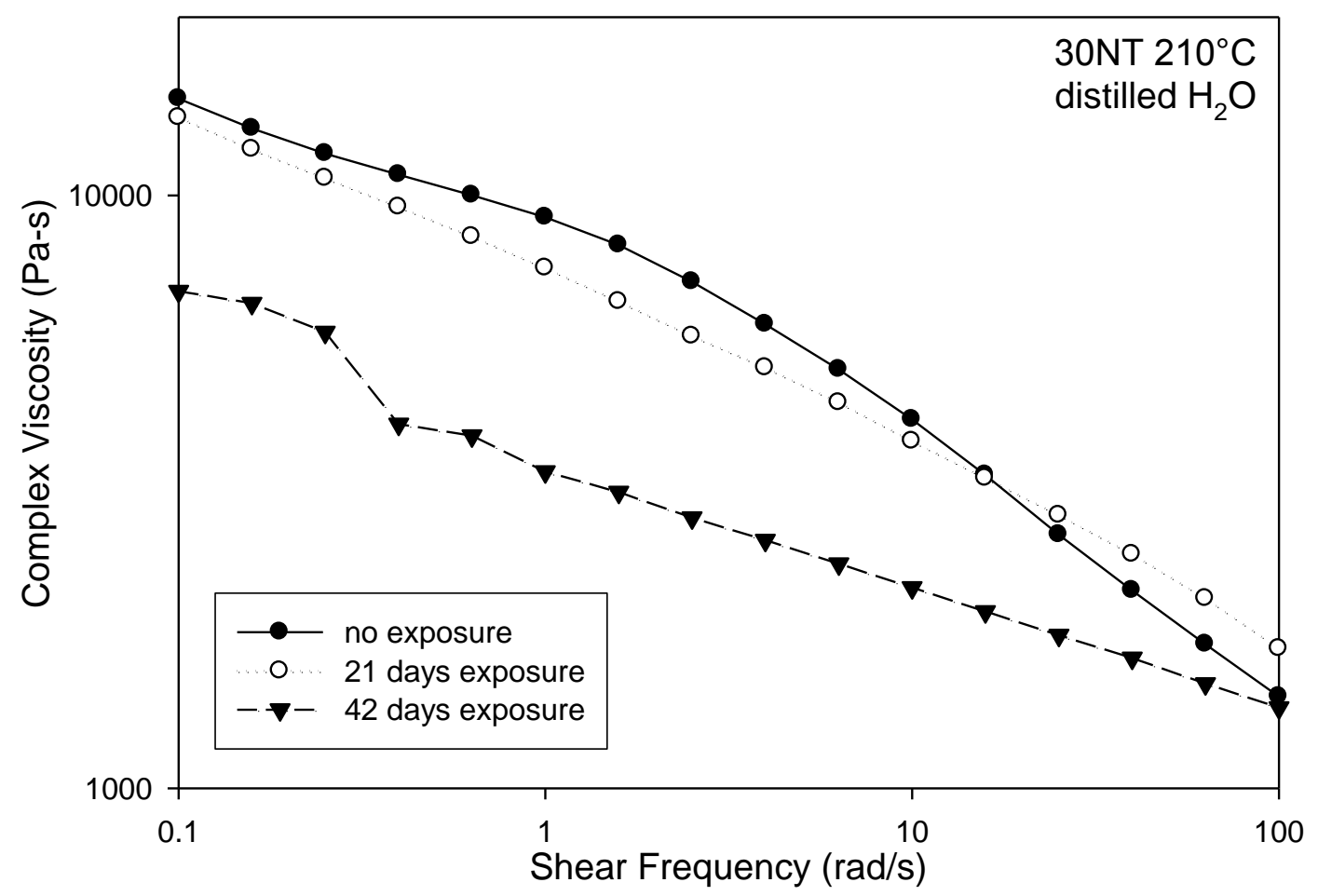

Figure 4.67: Viscosity change of sample $30 N T$ after distilled water degradation.

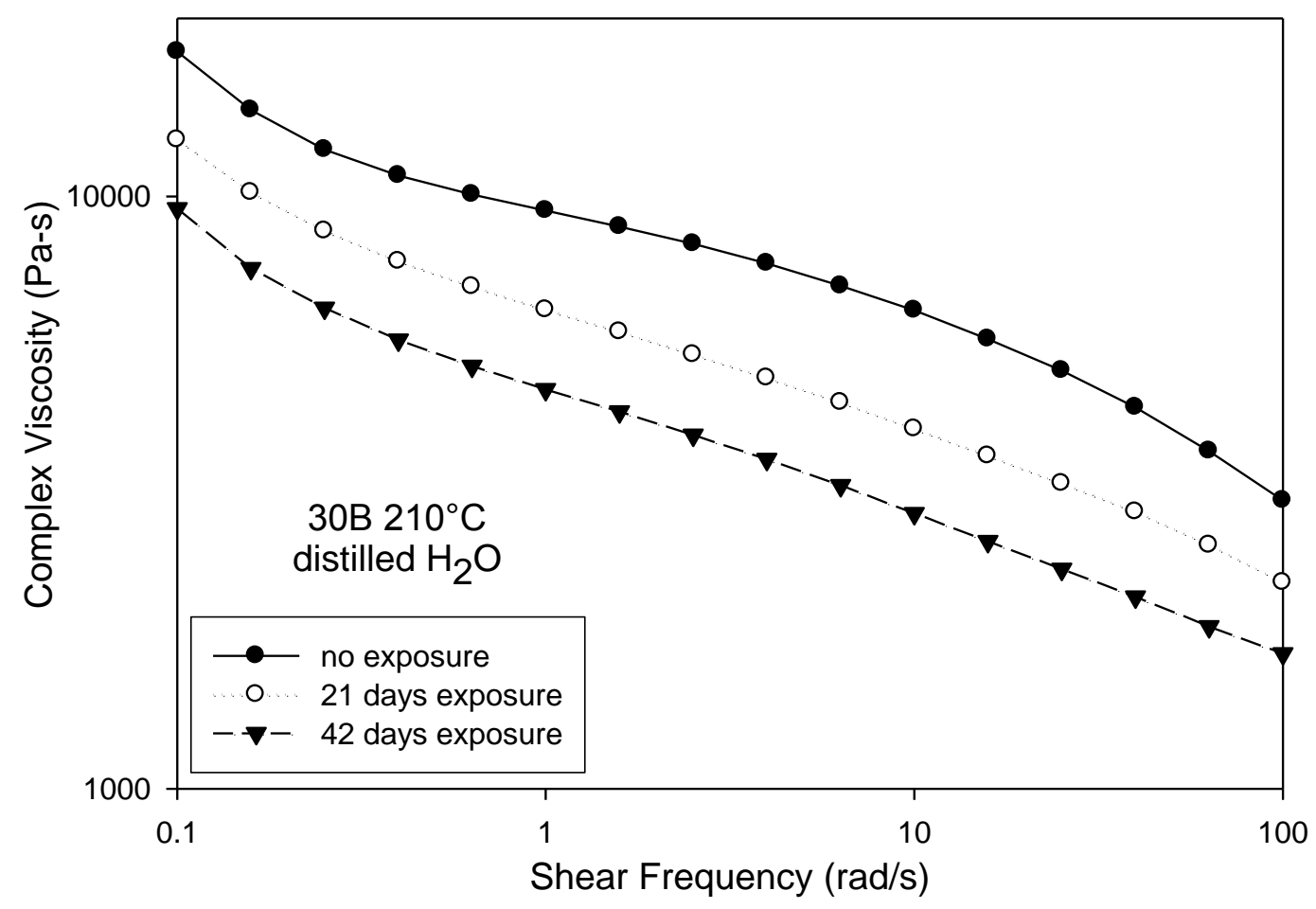

Figure 4.68: Viscosity change of sample $30 \mathrm{~B}$ after distilled water degradation. 


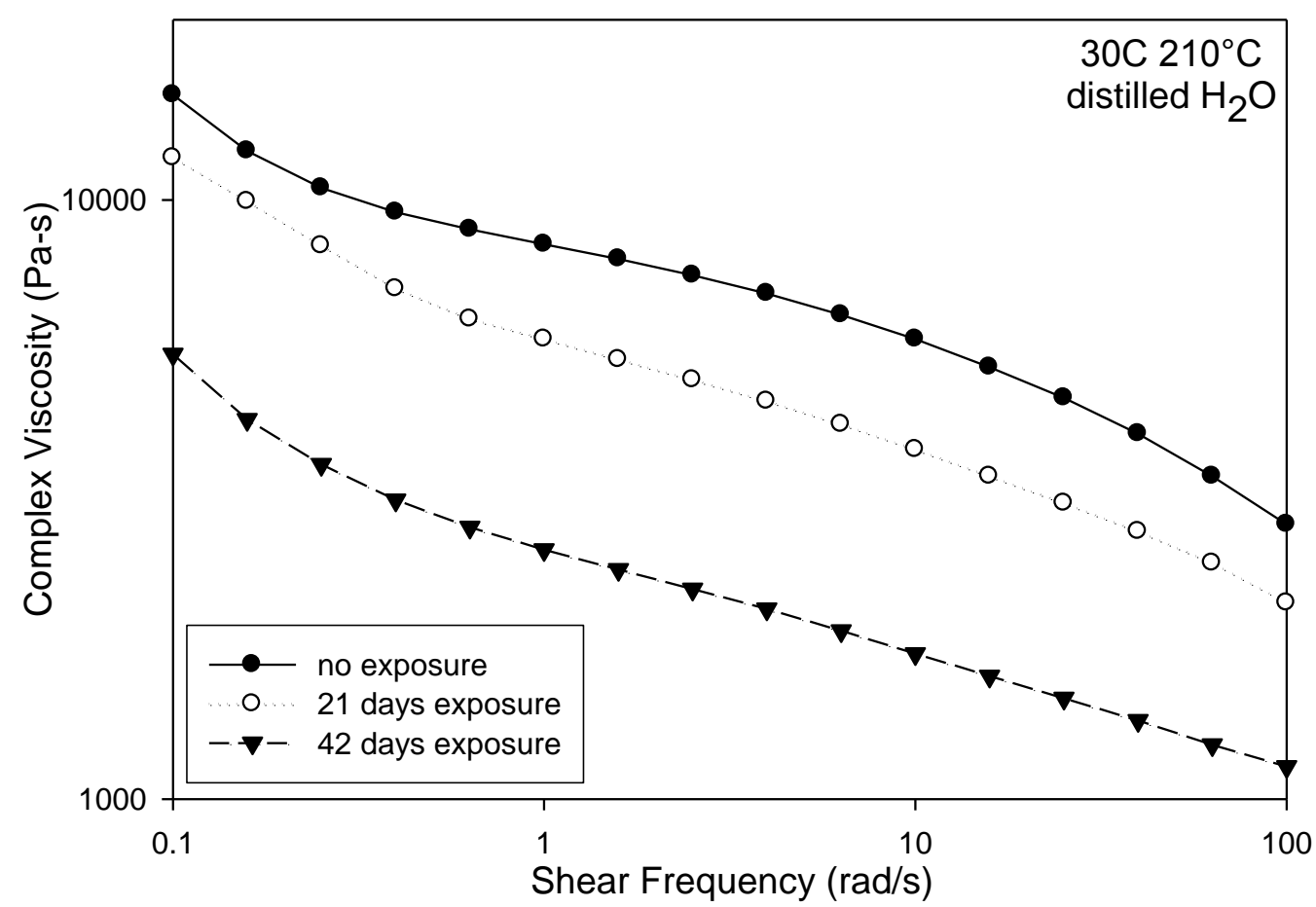

Figure 4.69: Viscosity change of sample $30 \mathrm{C}$ after distilled water degradation.

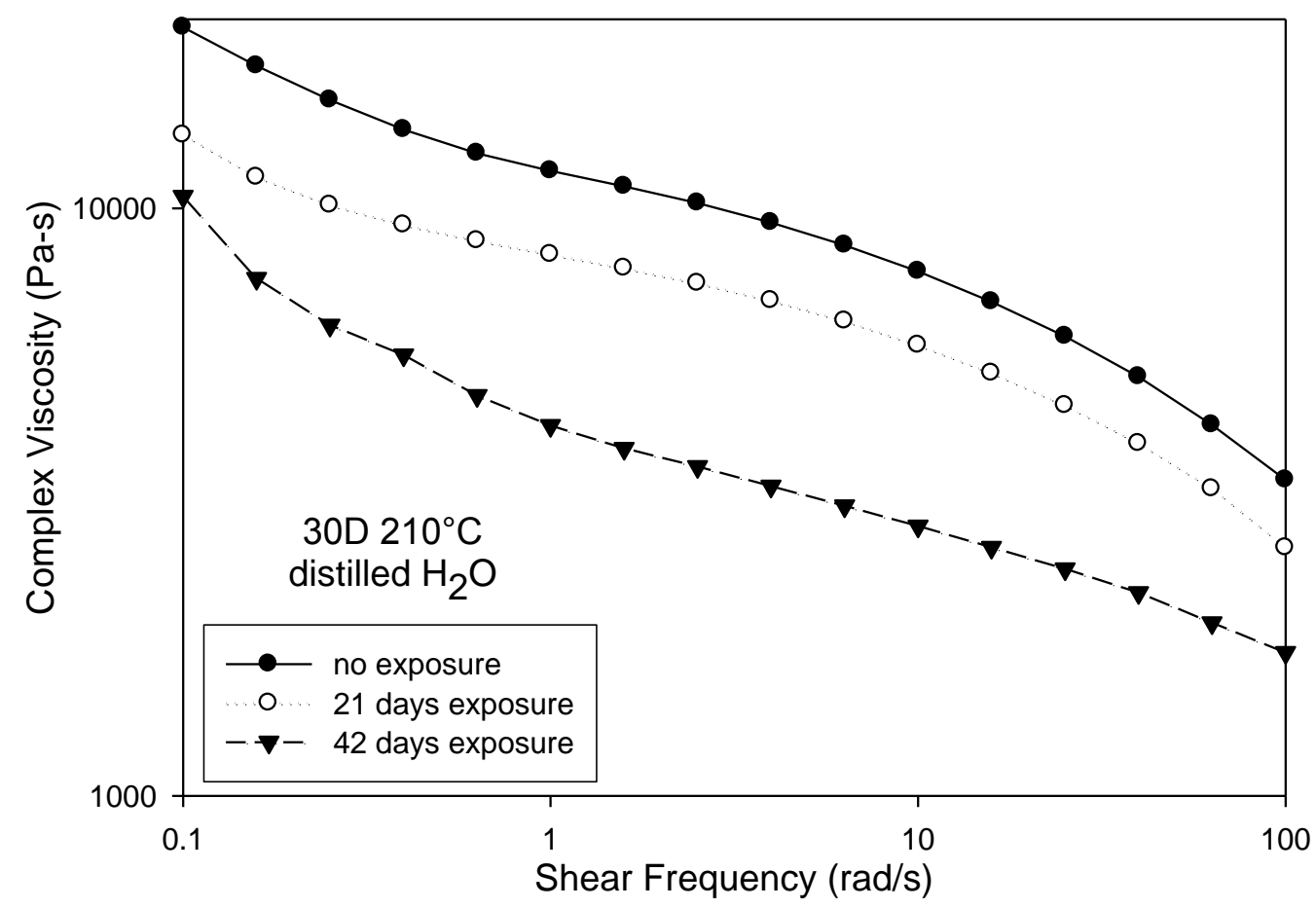

Figure 4.70: Viscosity change of sample $30 \mathrm{D}$ after distilled water degradation. 


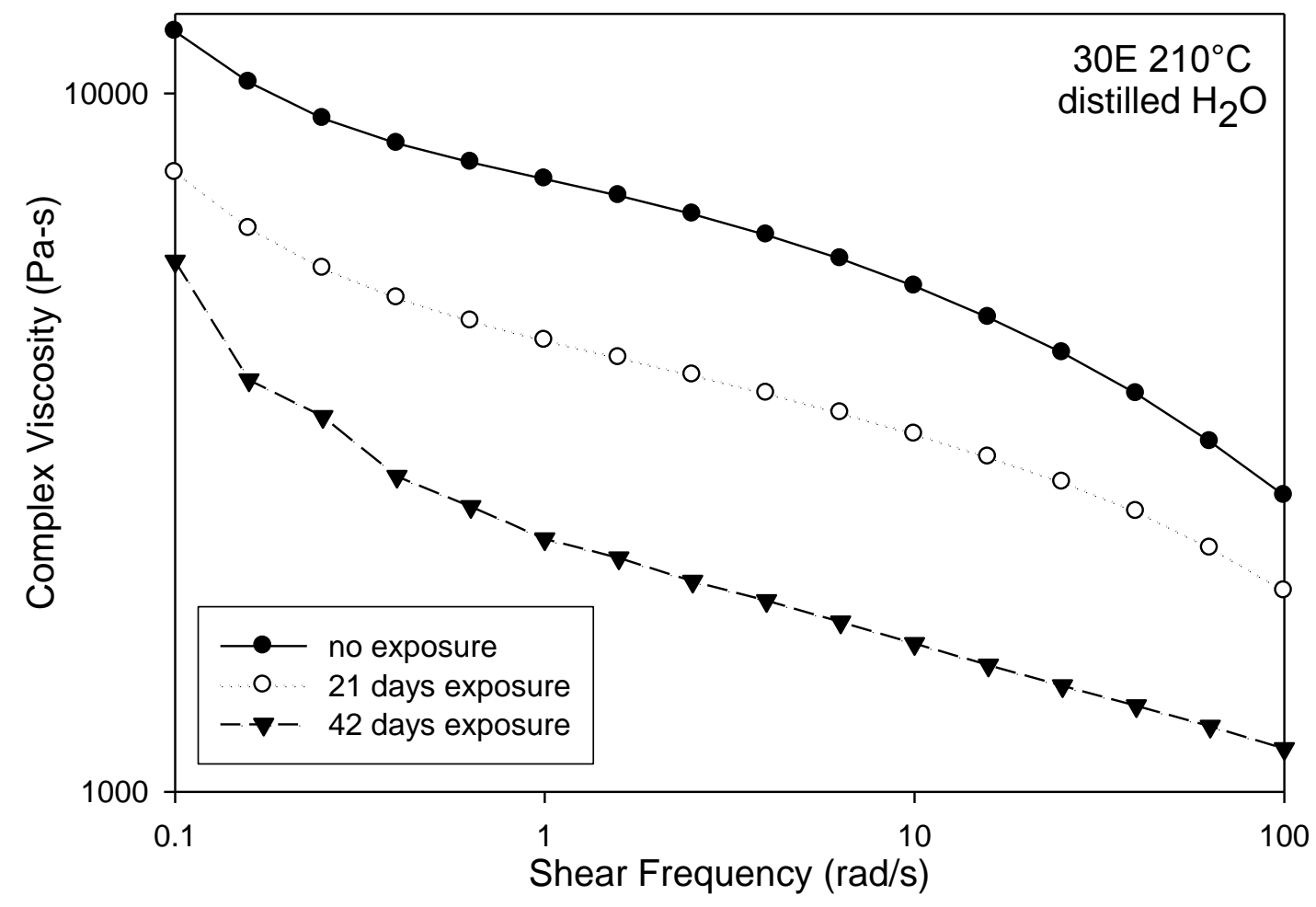

Figure 4.71: Viscosity change of sample $30 E$ after distilled water degradation.

From these figures it is not clear which material has the best viscosity retention, so we plot again the viscosity of all at $1 \mathrm{rad} / \mathrm{s}$ shear frequency in Figure 4.72: 


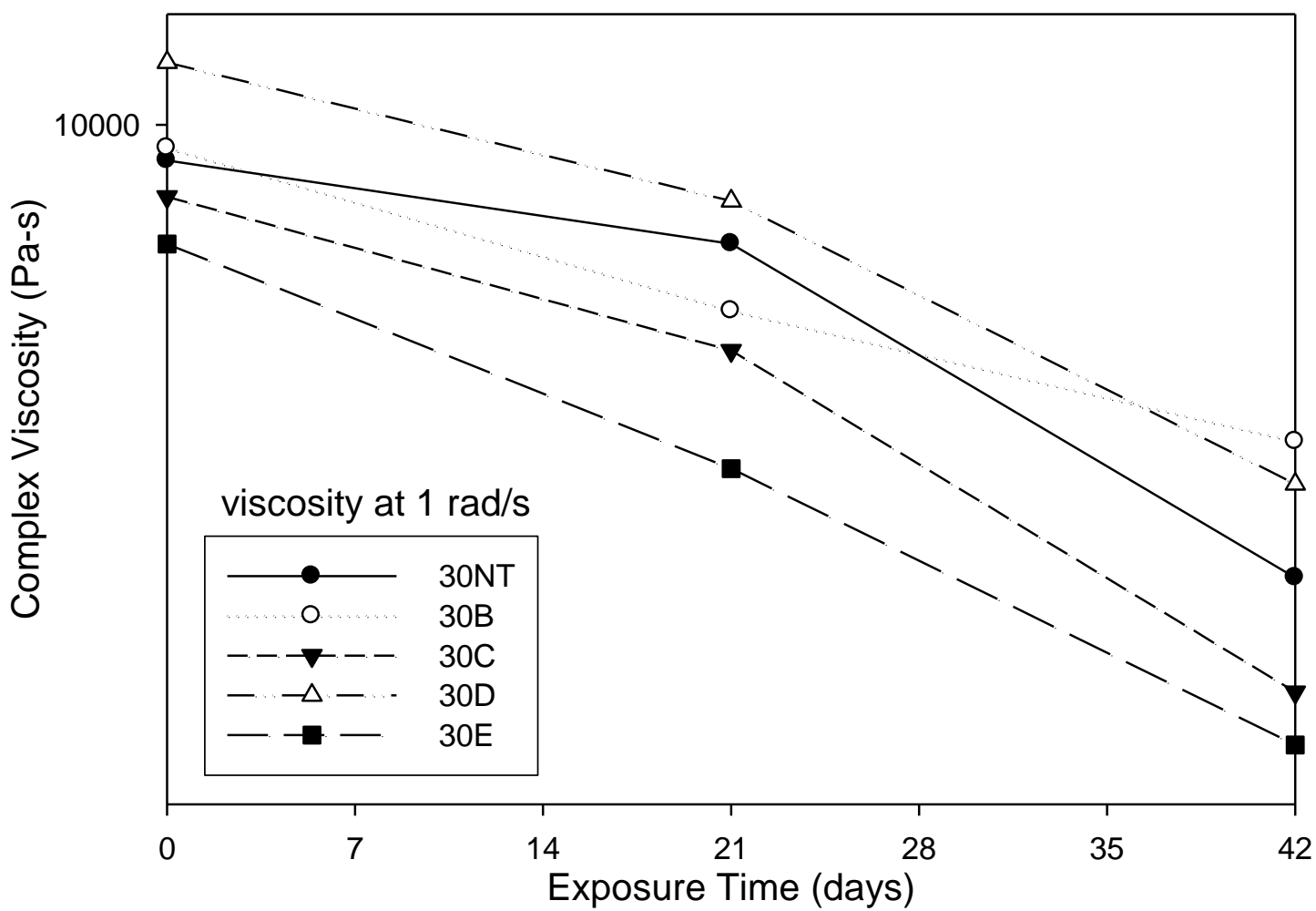

Figure 4.72: Comparison of viscosity loss between 30\% PLA blends of different internal morphologies.

Here we can see that there is not a great deal of difference between any of the blends, but it is clear that sample $30 B$ and $D$ have the best viscosity retention by the end of 42 days aging and sample $30 E$ has performed the worst. Recalling Table 4.7 , we observe while $30 B$ has the $2^{\text {nd }}$ largest droplets and $2^{\text {nd }}$ smallest relative area, the next best performing materials $30 D$ have the $2^{\text {nd }}$ smallest drops and $30 N T$ has the smallest droplets. The viscosity loss is tabulated here as it was in Table 4.4 for the blended materials:

Table 4.9: Viscosity loss at $1 \mathrm{rad} / \mathrm{s}$ after 42 days aging for the 30\% PLA blends of different internal morphologies.

\begin{tabular}{|l|r|}
\hline \multicolumn{1}{|c|}{ Sample } & Viscosity loss $(\boldsymbol{\%})$ \\
\hline 30NT & -62.8 \\
\hline 30B & -32.0 \\
\hline 30C & -69.1 \\
\hline 30D & -63.2 \\
\hline 30E & -69.5 \\
\hline
\end{tabular}


Since these results are clearly counterintuitive, it may be the case that the particle sizes are either too similar to have a profound difference in stability or that the particle size does not in fact matter; compiling the additional data will help explain these observations. A speculation could be made at this point that the materials with the best viscosity have been those that were processed the least. It is well known that excessive shear and thermal histories can degrade polymers, but more data need to be examined to verify this claim.

The next data to examine is the effect of hydrolysis on flexural properties and melt flow.

\subsubsection{Hydrolytic Degradation of Fully Encapsulated PC/PLA Blends.}

The initial mechanical properties of the encased PLA blends are given in Figures 4.73-75:

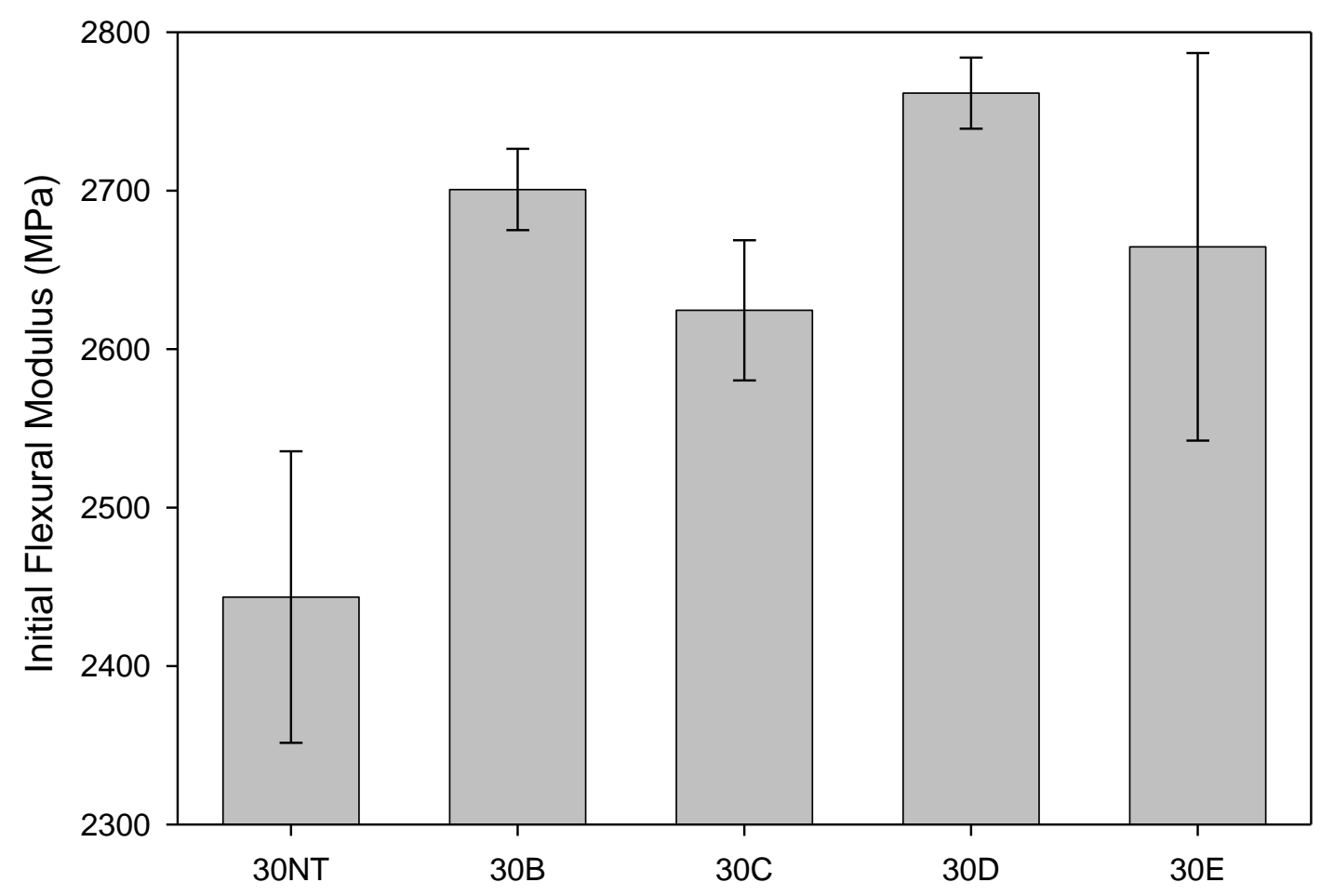

Figure 4.73: Initial flexural modulus of the encapsulated 70/30 PC/PLA blends. 


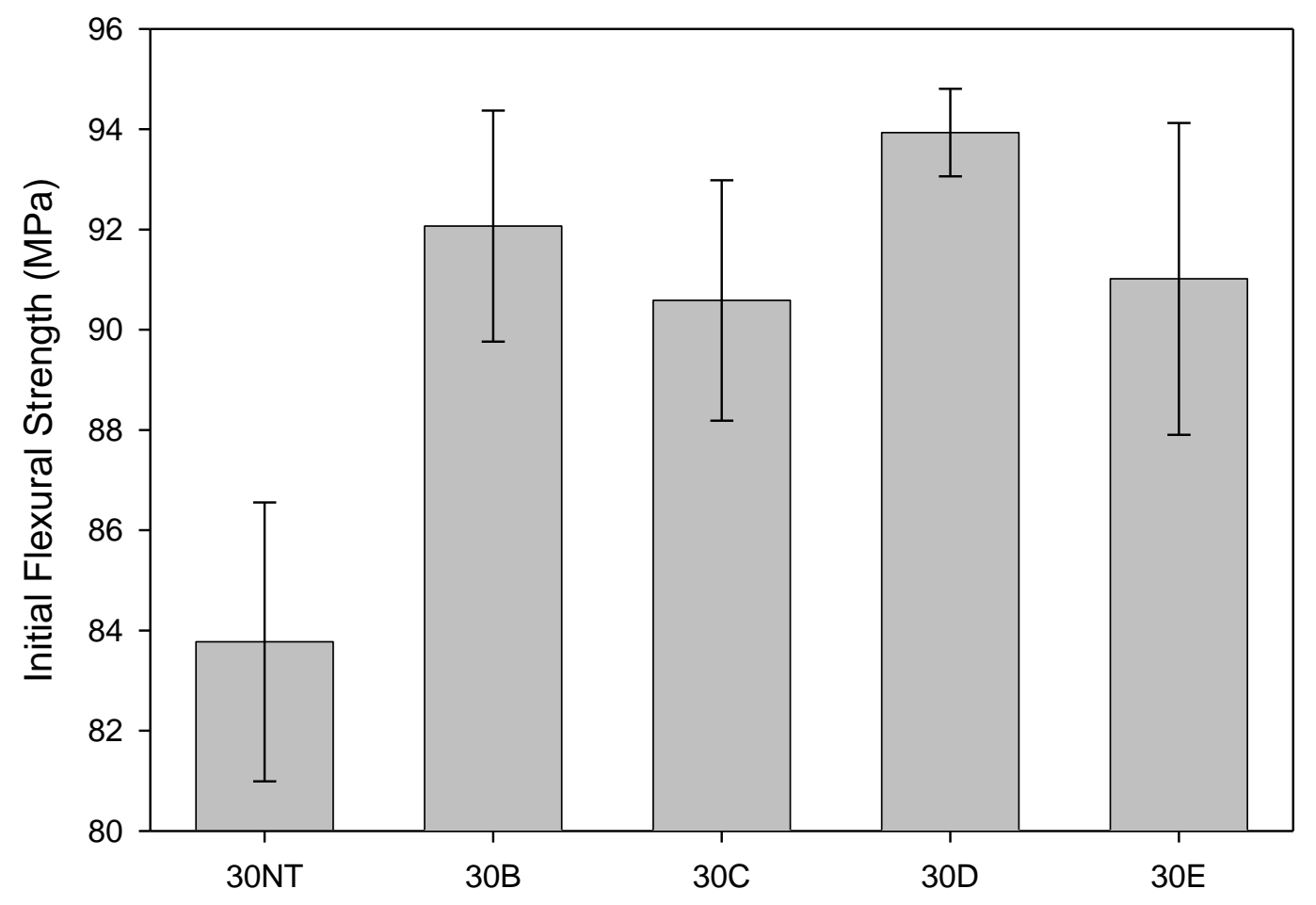

Figure 4.74: Initial flexural strength of the encapsulated 70/30 PC/PLA blends.

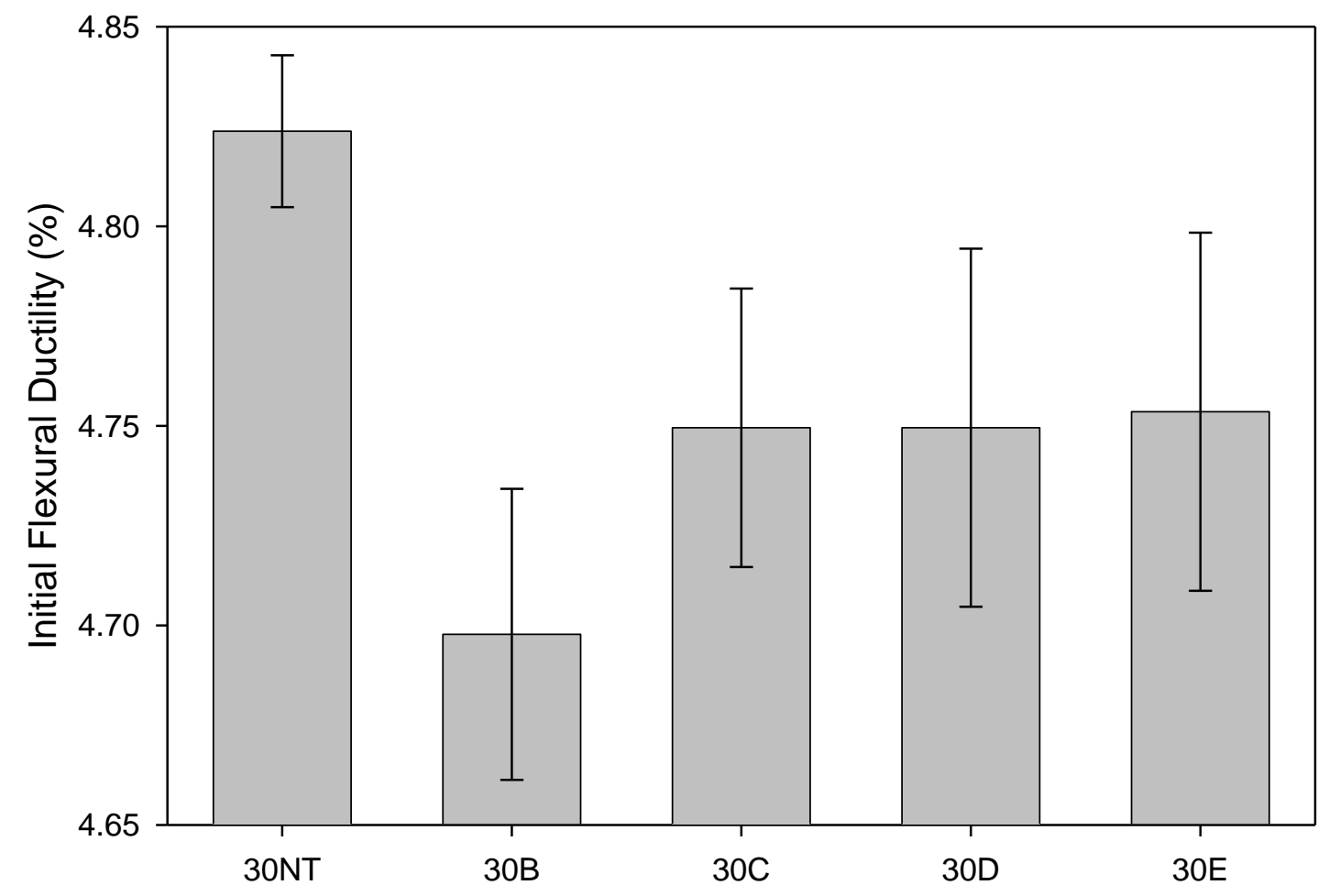

Figure 4.75: Initial flexural ductility of the encapsulated 70/30 PC/PLA blends. 
It is clear from the error bars that those materials internally mixed are statistically no different from each other. Single-factor ANOVA was performed on this data using a built-in Excel toolkit and showed that only the ductility of the extruded material 30NT was statistically different from the other data. Therefore, we can say the materials have roughly identical initial properties.

These molded bars were aged for up to 42 days to simulate 1 year of outdoor exposure. The flexural strength retention of the materials is given here in Figure 4.76:

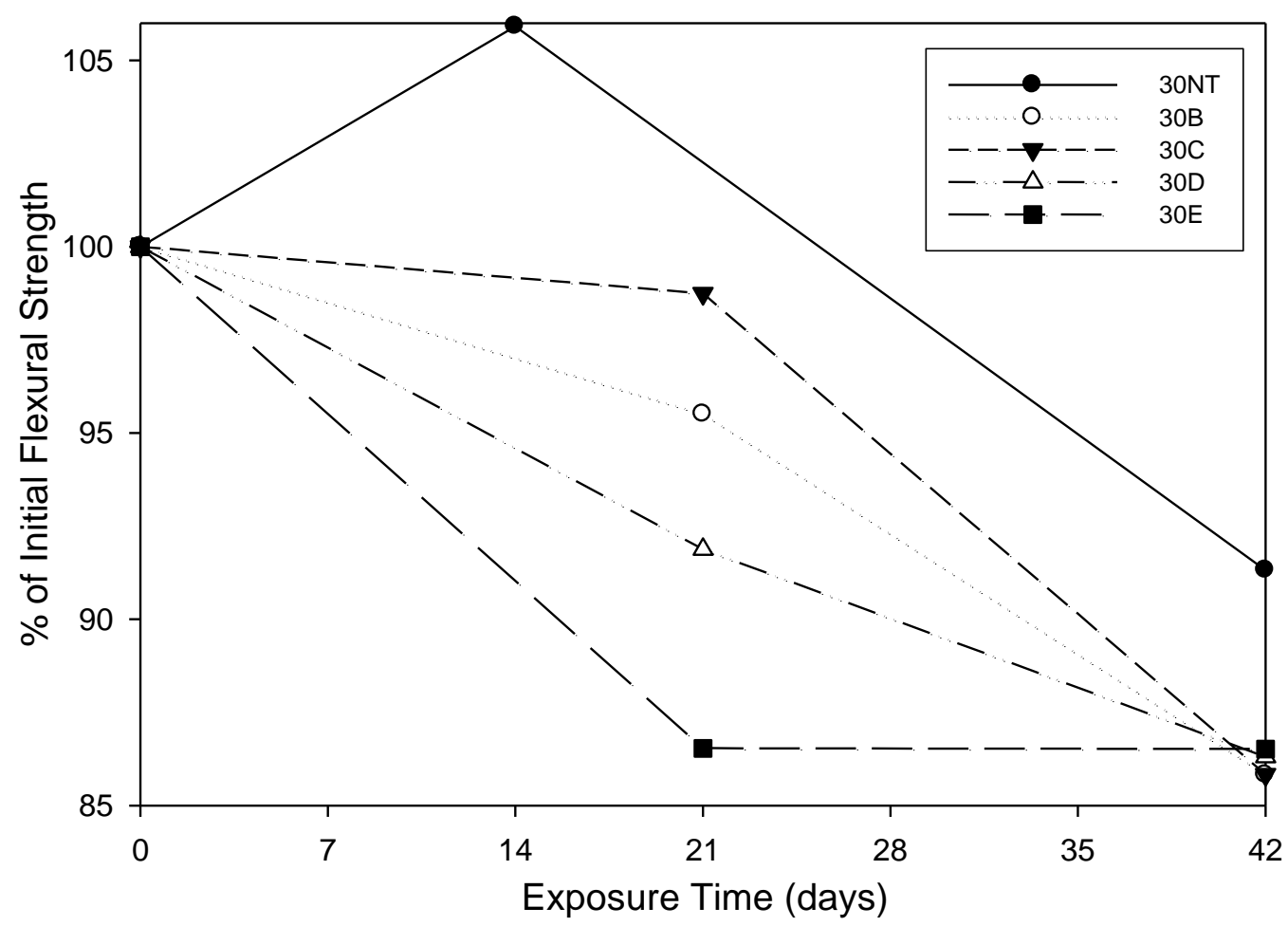

Figure 4.76: Flexural strength loss of encapsulated 70/30 PC/PLA blends of different internal morphologies.

After the 42 day exposure, the materials had about $85 \%$ at worst and $92 \%$ at best of the initial flexural strength remaining, a very good result if compared to any PLA-rich composition. In the worst case, samples $30 N T, 30 \mathrm{C}$ and $30 \mathrm{D}$ had ductility reduction to about $75 \%$ of initial after the 42 day period. These results also do not comport with the hypothesis about interfacial surface area as $30 N T$ had the smallest particles and $30 \mathrm{C}$ had the largest. Therefore, it cannot be said based on these results that the droplet morphology has played a role on the degradation resistance thus far. However, all the samples had superior resistance compared to any PLA-rich 
composition and even the worst performing blends still retained properties fairly well after 1 year equivalent aging.

Next, pellets of the fully encapsulated blends were subjected to hydrolysis for up to 7 days. The absolute value of the melt flow indices is plotted in Figure 4.77:

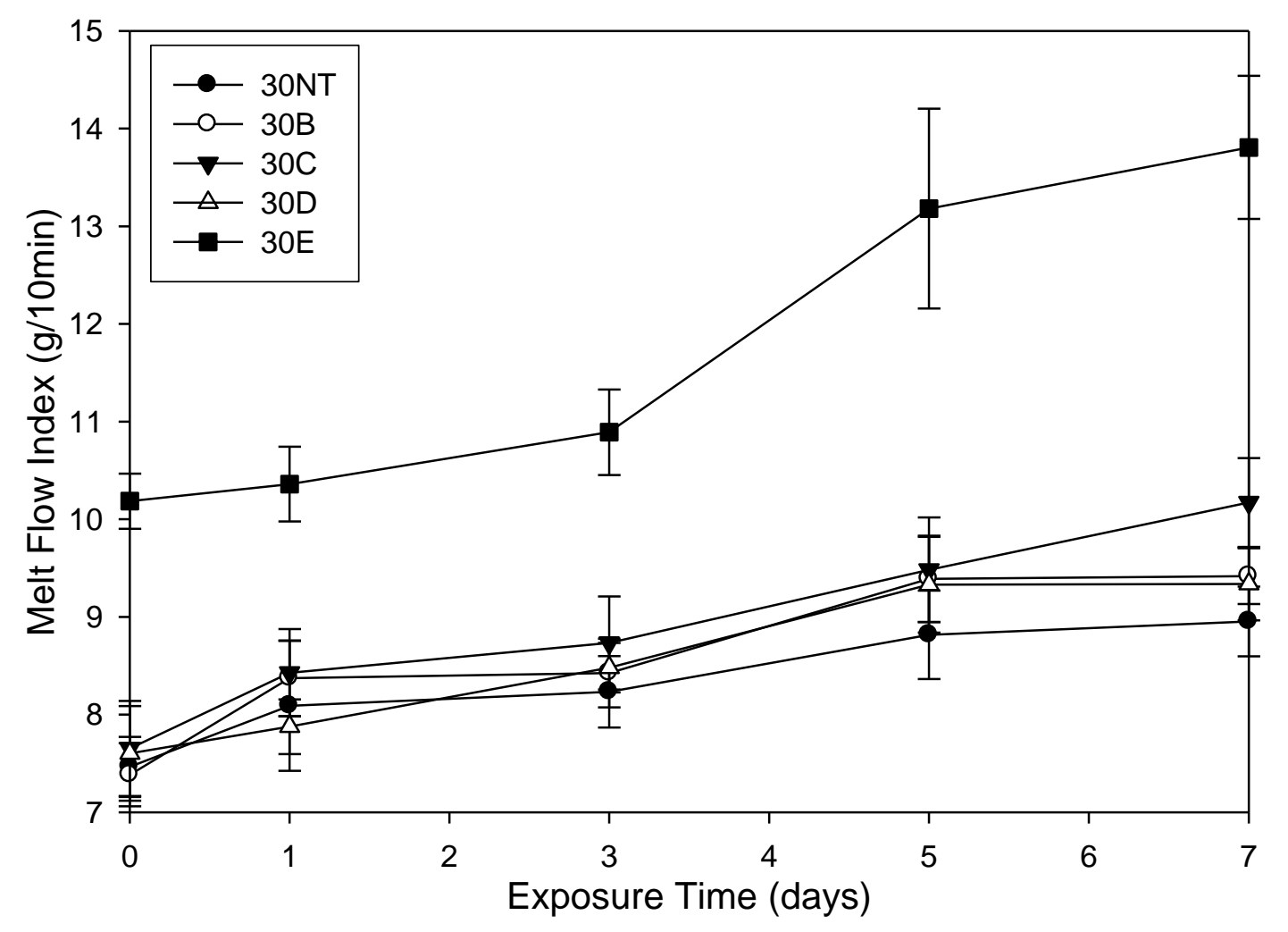

Figure 4.77: Increase in MFI of extruded and internally mixed encapsulated 70/30 PC/PLA blends.

While it is clear that sample $30 E$ processed at the most aggressive conditions has the largest MFI (and hence lowest viscosity), the remaining materials are similar with initial values of roughly 7 $\mathrm{g} / 10$ minutes increasing up to about 10 after 7 days exposure time. The percentage change will give additional information, shown in Figure 4.78: 


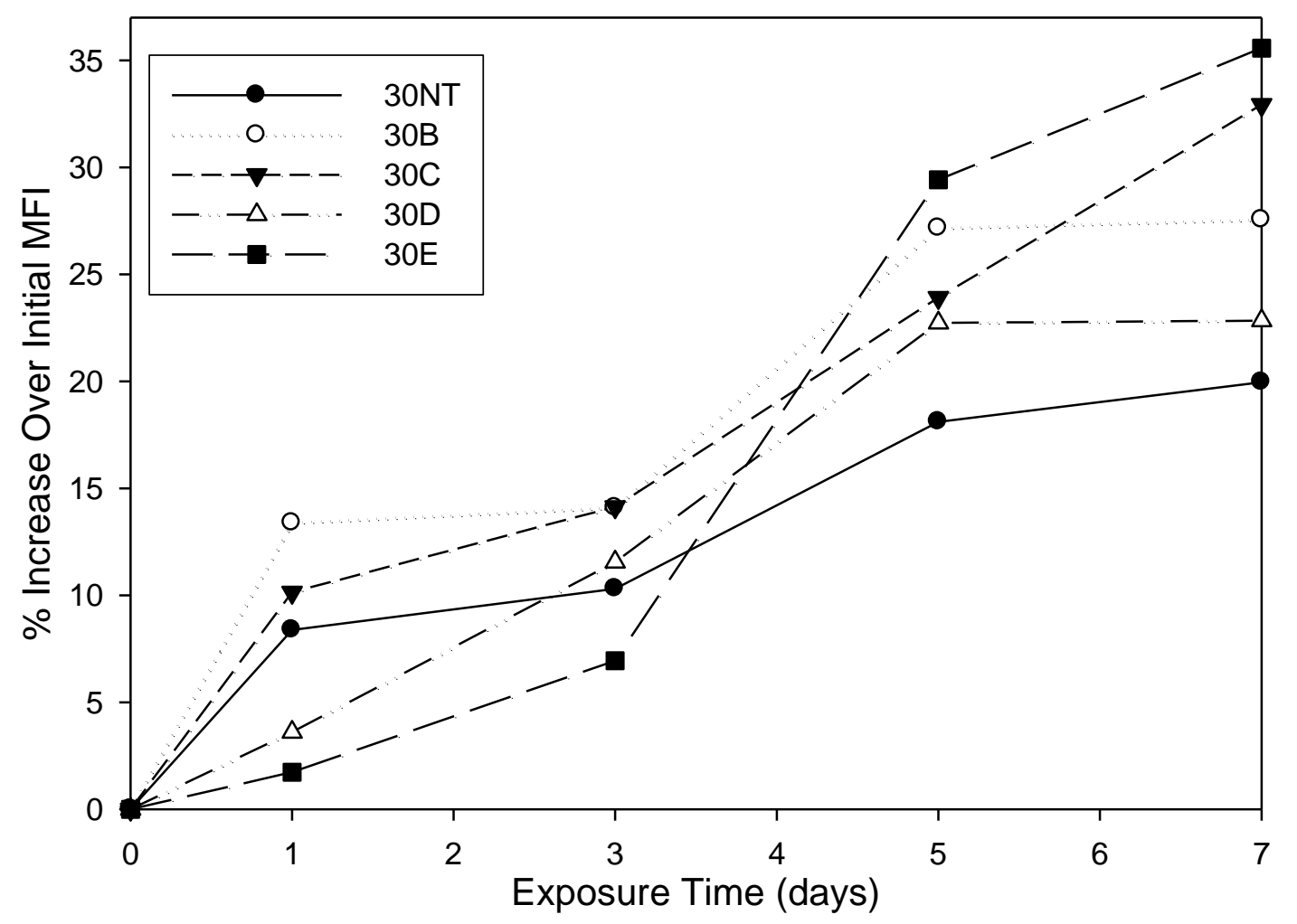

Figure 4.78: Normalized change in MFI for extruded and internally mixed encapsulated 70/30 PC/PLA blends.

Viewed in this light, the extruded material has the lowest increase followed by samples $30 \mathrm{D}$ and $30 B$, which are similar results obtained from the viscosity reduction plots. This seems to suggest strongly that in the circumstances investigated here, the effect of thermal and shear history from the compounding step has outweighed the effect of internal droplet morphology.

\subsection{Commentary on Fully Encapsulated 70/30 PC/PLA Blend Durability.}

In this section we have compared a number of blends in which $70 \%$ polycarbonate phase was used to completely encase 30\% PLA into discontinuous droplets throughout the PC matrix. It was hypothesized that alteration of the droplet structure to minimize their total area would be an avenue to increase the blends' durability. Investigation of the structure showed volume average particle sizes to range between about 1.3 to about $5.9 \mu \mathrm{m}$ in diameter, which would correlate to a sizable difference in interfacial area. However, degradation experiments did not show clear evidence that morphology played a role in increasing diffusion resistance or minimizing degradation. While it may be possible that there was not a significant enough difference in morphologies to realize any effect, there is stronger evidence that in this regime the effect of 
thermal and shear history plays a larger role than structure, so long as the PLA remains totally encapsulated by the more stable polycarbonate phase.

The final analysis is to examine the effect of increasing diffusion resistance by creating specimens of varying thickness. Like the annealing treatment and inclusion of graphene

previously, increasing the part thickness to retard diffusion will only be a transient effect. However, it is one we wish to quantify.

\subsection{Effect of Specimen Thickness on PC/PLA Blend Durability.}

Diffusion can be thought of most simply as the action of mass transfer through molecular movement rather than by means of velocity such as with advection. This action is known to occur fairly rapidly in either gases or liquids but is slower in solids. Thus, by increasing the dimensions of a solid specimen, there is additional material present through which attacking species or media must diffuse through in order to reach the centerline of the object.

Let us consider a flexural bar exposed to the humidity chamber as an example. A representation of this situation is given in Figure 4.79: 


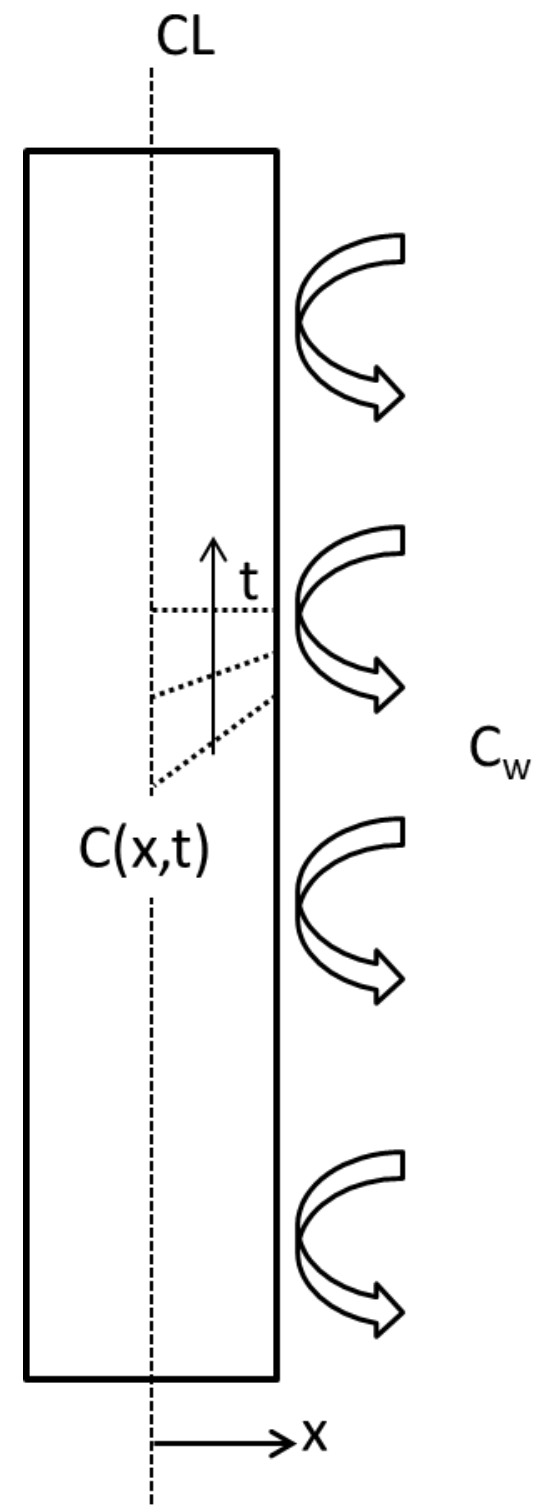

Figure 4.79: Representation of flexural bar exposed to humidity chamber.

There is some concentration of water vapor $C_{w}$ that the surface of the bar is exposed to, which will gradually penetrate into the body of the bar in the $x$-direction as time goes on. In this case, the aspect ratio of the bar is large so we can make an approximation that the surface is the dominant area over which diffusion takes place and we can ignore the ends and sides. There will be a concentration profile inside the bar that is a function of time and position. The boundary conditions for this situation are: 


$$
\begin{aligned}
& C(x, t=0)=0 \\
& C(x=L, t)=C_{w} \\
& \frac{\partial C}{\partial x}(x=0, t)=0
\end{aligned}
$$

Which describe that initially there is no moisture inside the bar, the surface concentration is always equal to that of the humidity chamber (making the assumption that whatever moisture that penetrates into the bar does not reduce the concentration of moisture in the air) and that the concentration profile inside the bar is symmetric. The concentration profile for similar cases has been solved previously (e.g ${ }^{99}$, and is given below):

$$
\frac{C}{C_{w}}=2 \sum_{0}^{\infty}\left(-1^{n)} \operatorname{erfc}\left(\frac{x(2 n+1)}{\sqrt{4 D t}}\right)\right.
$$

Where $x$ is the thickness of the bar, $D$ is the diffusion coefficient of water in the polymer and the complementary error function can be found in any mathematics textbook. Literature suggests that the diffusion coefficient for moisture in 95/5 L/D PLA is approximately ${ }^{100} 0.2 * 10^{-6} \mathrm{~cm}^{2} / \mathrm{sec}$ and is about the same for $\mathrm{PC}^{101}$ at $50^{\circ} \mathrm{C}$ and $90-100 \%$ relative humidity. Using these values, we can calculate that the time required for the centerline of the bars $(0.127 \mathrm{~cm}$ for thin, $0.238 \mathrm{~cm}$ for thick) to reach $50 \%$ of the moisture concentration of the air/water vapor would be about 8.5 hours for the thin bar and 29.7 hours for the thick bar. Thus, there is a theoretical advantage in retarding diffusion by increasing the thickness of the bars, which was investigated experimentally.

\subsubsection{Comparison of Thin-Walled and Thick-Walled Flexural Bars.}

As mentioned in Chapter 3, two thicknesses of flexural bars were compression molded. Steel molds of nominal thickness $1 / 10$ and $3 / 16$ inches (2.54 and $4.76 \mathrm{~mm}$, respectively) were used to cast these bars, which were then subjected to hydrolysis for up to 42 days exposure. The initial properties of the thin and thick bars are given in Figures 4.80-82: 


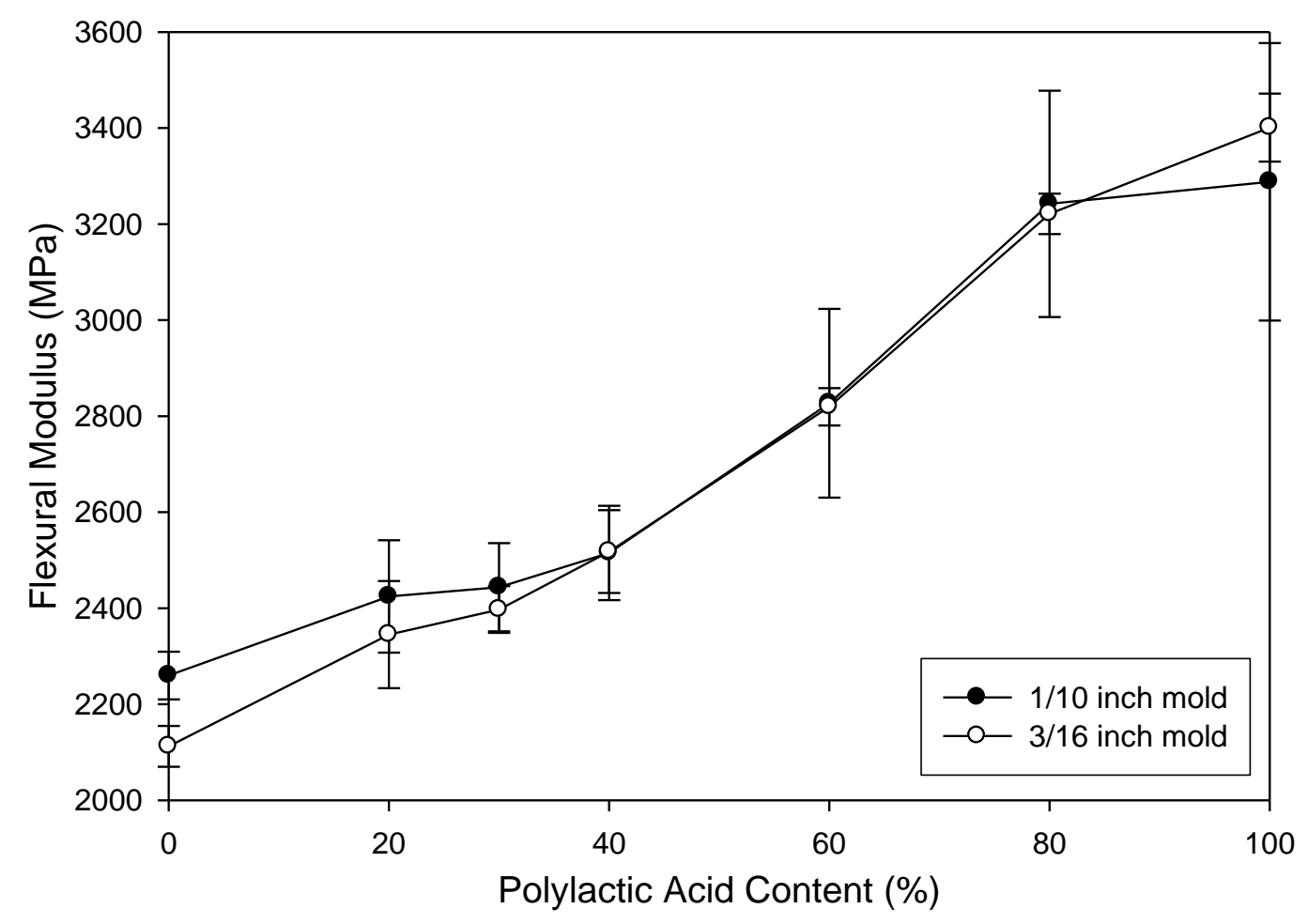

Figure 4.80: Initial flexural moduli of the thin and thick molded untreated PC/PLA blends.

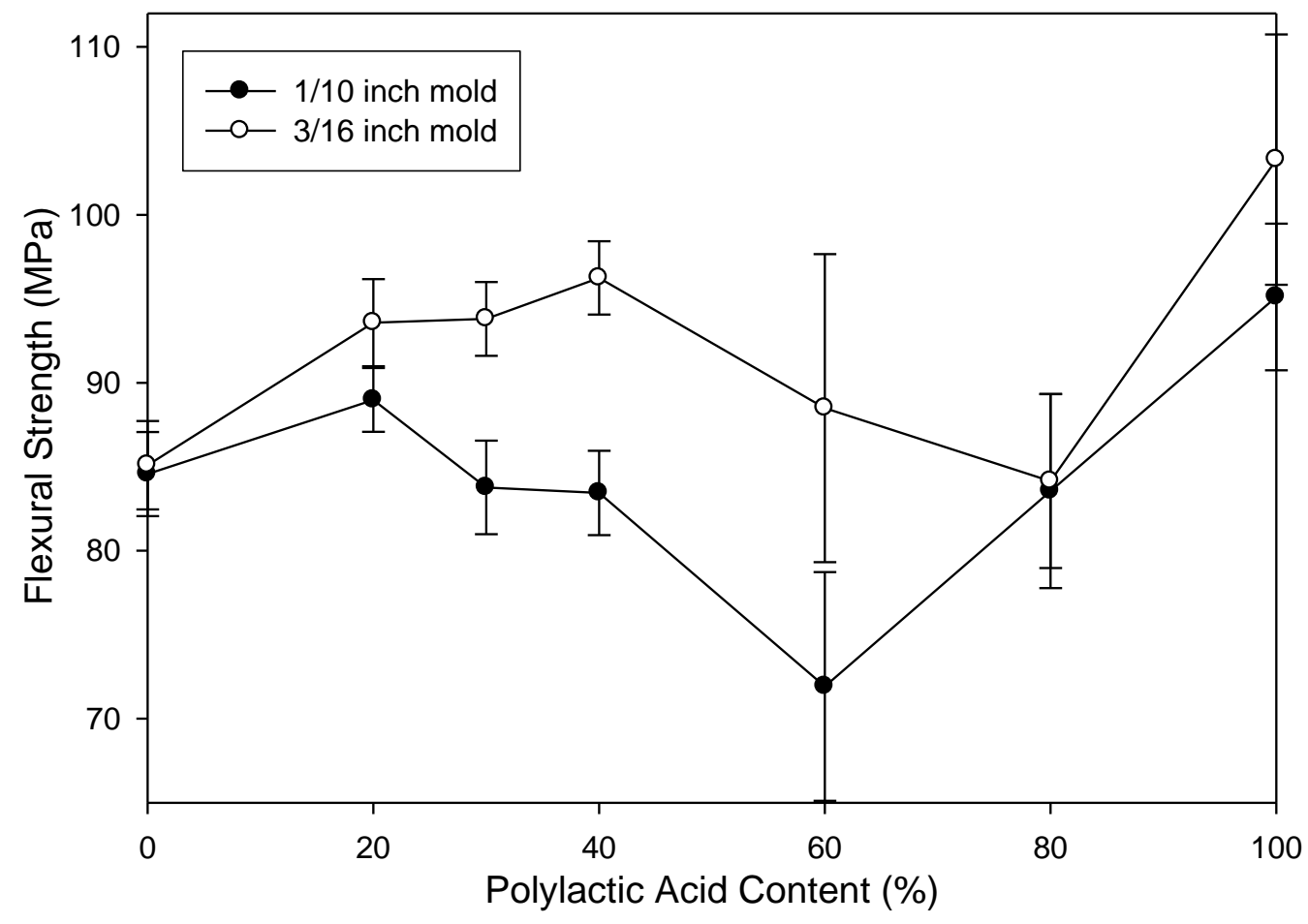

Figure 4.81: Initial flexural strength of the thin and thick molded untreated PC/PLA blends. 


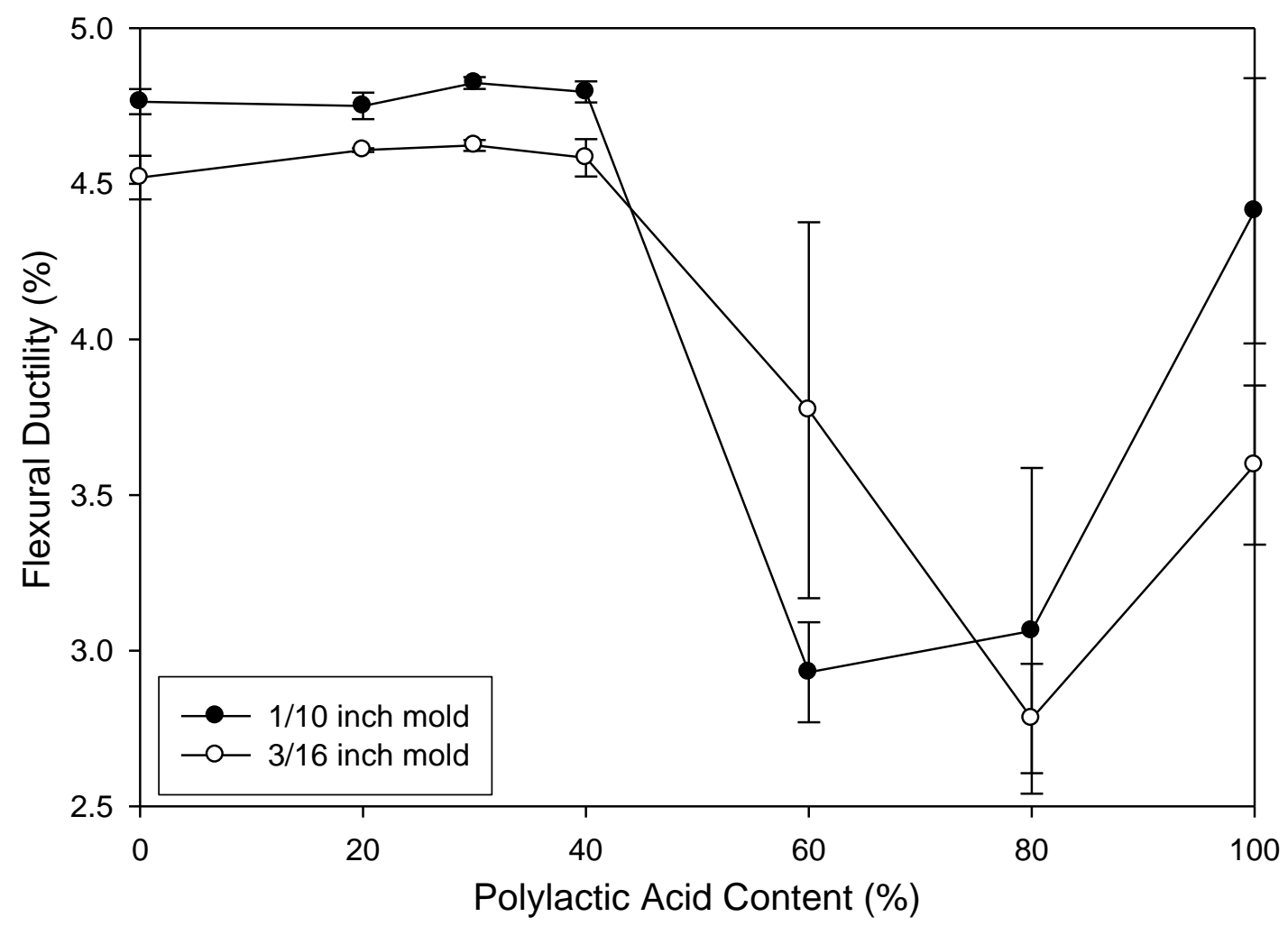

Figure 4.82: Initial flexural ductility of the thin and thick molded untreated PC/PLA blends.

Like previous samples, the flexural modulus appears to be linearly proportional with composition for both thin and thick samples, while the strength and ductility suffer at $60-80 \%$ PLA concentration. It is likely in these cases that the PC phase for these formulations are acting as impurities or stress concentrators (much like large aggregates of graphene would) which damage the mechanical properties. Comparing Figure 4.81 with 4.82, the thicker materials have slightly higher flexural strength and slightly lower ductility than the thinner bars; it is known that part thickness can have an effect on mechanical properties. It is also possible that once parts reach a critical thickness, the failure mode can change from ductile to brittle (e.g. $\left.{ }^{102}\right)$. This is related both to sample thickness and rate of mechanical loading, which suggests this critical point is where the material can no longer deform as rapidly as stress is applied and therefore brittle fracture occurs. In this work, however, that limit has not been reached.

In the following plots, bars made from materials $100 N T$ and $80 N T$ are omitted as these underwent severe degradation so rapidly that long-duration analysis was not performed. 
Therefore, the change in flexural strength and ductility are shown for untreated flexural bars containing 60\% PLA phase or less in Figures 4.83-84:

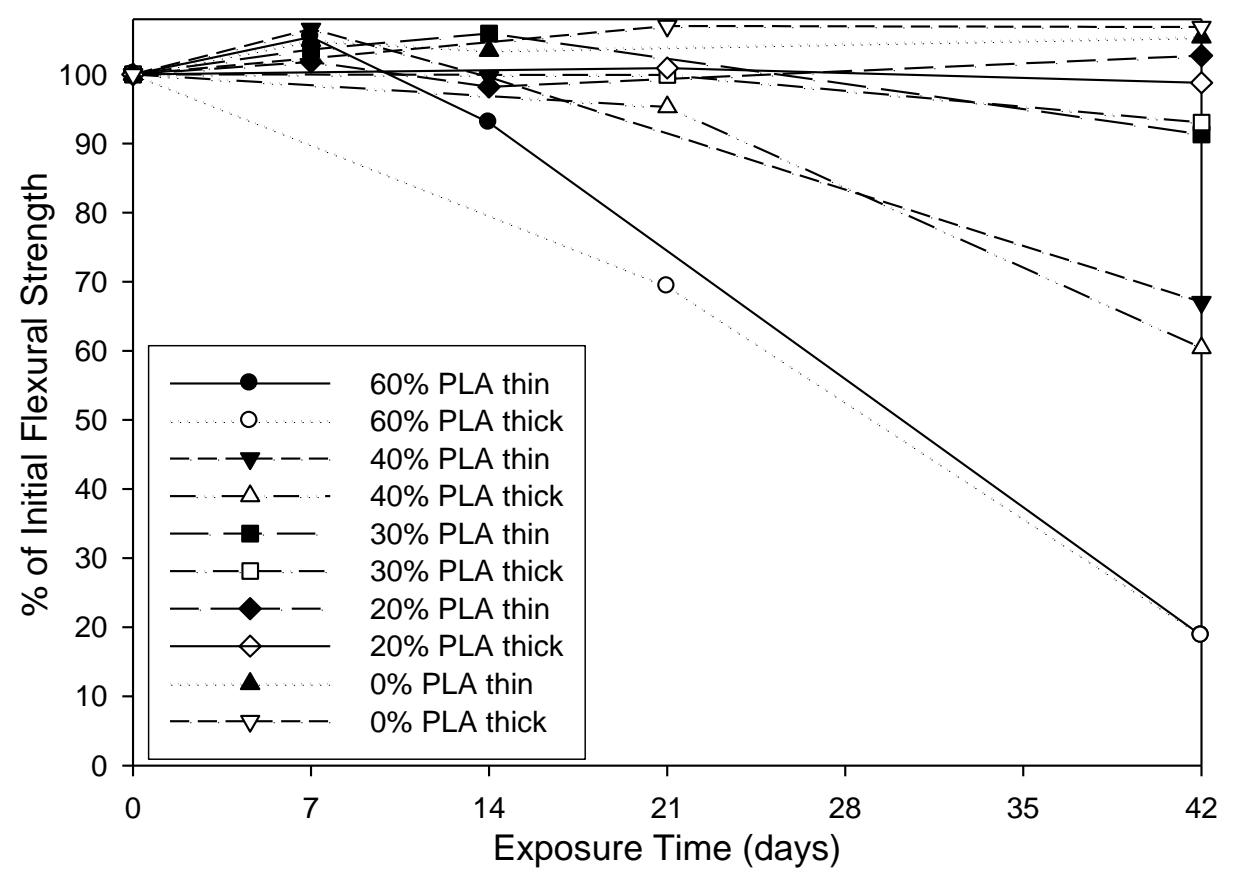

Figure 4.83: Flexural strength loss of thin and thick molded untreated PC/PLA blends.

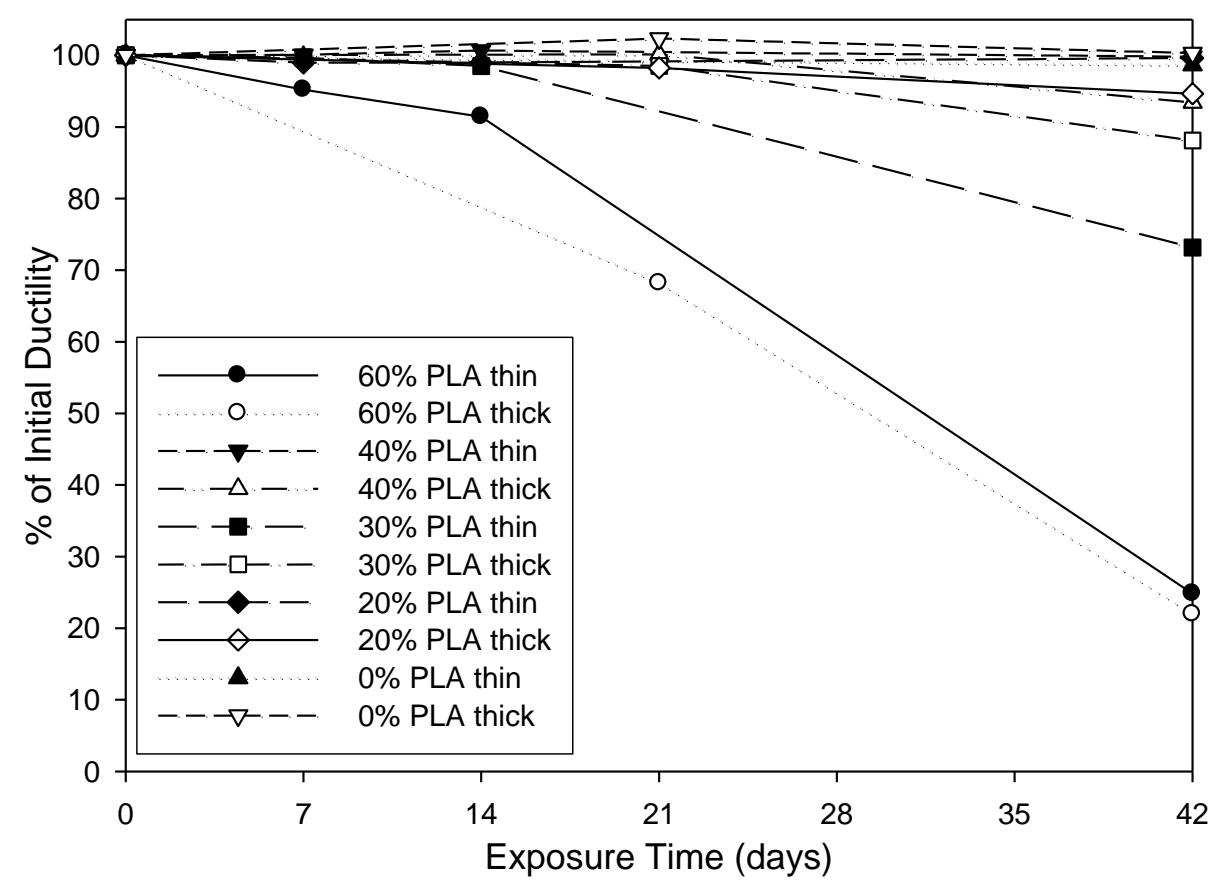

Figure 4.84: Flexural ductility loss of thin and thick molded untreated PC/PLA blends. 
The 60NT samples in both cases showed tremendous property losses by the end of 42 days degradation, which confirms that any PLA-rich composition is too unstable for a durable application. Here, there only appears to be an improvement visible with the ductility loss of the thin vs. thick $30 N T$ composition after 42 days; as the amount of PLA is decreased the effect of thickness should be reduced. In the other cases the effect of increasing thickness appears to be negligible. It is entirely plausible that by using a wider range of dimensions some significant effect of diffusion resistance may appear, but across this thickness range from roughly 2.5 to 4.5 $\mathrm{mm}$ no appreciable difference was observed, as the exposure times were much longer than the calculated time for significant diffusion to occur. Therefore, our previous speculation that the rate of diffusion is much faster than the hydrolysis reaction seems to hold true; furthermore, since the diffusion coefficient for both PC and PLA has been published to be the same, this would also explain why the effect of interfacial areas for the fully encapsulated blends was minimal. Therefore, if one wishes to attempt this avenue of creating more durable composites two caveats must be addressed, as parts would need to become exceedingly thick: materials will behave unexpectedly if the critical ductile-to-brittle transition is exceeded and since both PC and PLA are fairly dense polymers (density around $1200 \mathrm{~kg} / \mathrm{m}^{3}$ ), part weight would quickly become a negative factor.

Having completed the degradation research across a large range of formulations and treatment options, we may now make conclusions and recommendations based on the findings. 


\section{CHAPTER 5}

\section{CONCLUSIONS AND RECOMMENDATIONS.}

\subsection{Conclusions.}

The research focus of this work was to create durable plastic materials and composites that contained significant renewable and ecologically friendly polylactic acid polymer. To complete the study, several techniques were employed; exploiting the crystalline nature of the PLA polymer to enhance properties and chemical resistance, adding barrier materials to retard diffusion and strengthen the material, blending in a sturdier plastic phase, and attempting to alter the interfacial morphology of PC/PLA blended material to provide more chemical resistance. As a result of undertaking these studies, we can make the following conclusions:

- Polylactic acid polymer by itself is simply not durable enough to withstand our tests aimed at replicating up to 1 year outdoor exposure. Because the material is derived from a low molecular weight ester monomer, the overall concentration of ester bonds in the backbone is quite high which leads to extensive hydrolysis over time.

- By annealing the PLA polymer or otherwise enhancing the crystallinity, the material becomes slightly more mechanically stable but less ductile. This is due to rearrangement of polymer chains into a more regular structure, which enhances intermolecular hydrogen bonding. These crystal structures provide better resistance to chemical attack than do amorphous segments by virtue of excluding species and ions from the crystal gallery spacing. However, as PLA polymer is fundamentally prone to hydrolysis and even the crystalline regions will break down over relatively short timescales, improving the crystallinity is a short-term solution and ceases to be effective once diffusion resistances are overcome. Additionally, annealing the materials either in the mold or post-molding creates a higher cost for the materials and therefore may be impractical.

- The addition of graphene nano-platelets at $2 \%$ weight loading also serves to slightly improve mechanical properties at the expense of ductility. Furthermore, we have shown that graphene can act as a nucleating agent for PLA crystallization which improves the crystallization kinetics but does not alter the amount of crystal growth or the dimensions 
of crystals that are formed. Then, unlike the crystalline PLA domains, the graphene sheets will not erode over hydrolytic exposure, providing a permanent barrier within the plastic composite. Unfortunately, species will still eventually diffuse around these barrier plates, and then the PLA phase will ultimately degrade. As such, the addition of graphene is also a short-term solution.

- By compounding the PLA polymer with polycarbonate, a wide variety of compositions were created. As was previously determined, the two phases are found to be thermodynamically immiscible. Image analysis showed that depending on composition, either phase could become the matrix or dispersed phase, or when blended in roughly equal proportions a co-continuous phase would form. Addition of the PC phase was not shown to have an effect on the crystallization of PLA-rich blends, but difficulty was encountered when attempting to analyze the kinetics of materials containing less than $40 \%$ PLA. Degradation experiments showed that once the blended materials became PC-rich, they were much more stable over time. Ideal blends are created with the polycarbonate totally encapsulating the less stable PLA phase, which occur at PLA concentrations of 30 weight percent or less. Aging experiments up to 1 year outdoor equivalent showed these materials to have promising hydrolysis resistance and maintenance of physical properties.

- PC/PLA blends were compounded together in an internal mixer at constant 70/30 composition in order to evaluate the effect of droplet size and interfacial surface areas. Five different processing conditions were used which gave number average particle sizes ranging from about 0.8 to $1.7 \mu \mathrm{m}$, and volume average sizes from about 1.3 to $5.9 \mu \mathrm{m}$. The effect of morphology was not clear-cut based on evaluation of degradation results. It may be that the morphologies were not significantly different enough to observe any effects, or that since the diffusion coefficient for water moving through both PC and PLA is the same there may be no advantage in altering the morphology. The results seem to suggest that thermal and mechanical shear history play a more important role. Samples that received the most aggressive mixing history tended to be less durable in the face of degradation, likely due to molecular weight loss.

- By increasing the thickness of molded samples, a diffusional resistance can be added. While this does not alter the inherent stability of the material, it can provide a transient 
barrier which can enhance the usable lifetime of the formulations. In this work, we have found that the rate of diffusion into the polymer bulk is much faster than rate of PLA hydrolysis. For instance, the viscosity of all treatments of 98-100\% PLA was reduced to nearly zero after 42 days of exposure to solvents and distilled water at $50^{\circ} \mathrm{C}$. By using the complementary error function solution, it was determined that the time required for the concentration of moisture at the centerline of a $3 \mathrm{~mm}$ thick plaque to reach half the outside concentration was about 11.8 hours. Therefore, we can be confident in claiming that diffusion into the PLA bulk by water or other species is very fast with respect to the hydrolysis reaction.

- Based on these findings, it is recommended that materials must contain at minimum $70 \%$ polycarbonate matrix phase in order to maintain chemical and dimensional stability. These materials should also be compounded in a processing space which can adequately blend and disperse the PLA phase while minimizing thermal history and degradation. Lastly, since the PLA phase is dispersed in the ideal compositions, annealing and adding graphene in these composites can add an extra short-term boost in degradation resistance without causing unwanted embrittlement, but this effect is very small compared to total encapsulation by polycarbonate. Once this occurs, the material takes on the properties of the matrix phase.

We may also make a few recommendations for additional studies based on the information we have obtained.

\subsection{Recommended or Plausible Further Studies.}

The following concepts or topics may be of interest for future workers:

- Determine if a precise composition exists for maximum PLA content that is still fully encapsulated by the PC phase. Polycarbonate is a great deal more stable and has favorable mechanical properties as the matrix phase, especially ductility. Based on our findings with these particular raw materials, a maximum concentration could consist of between 30\%$40 \%$ polylactic acid by weight. This would likely be a difficult endeavor with the available extruder based on throughput and metering capability and therefore should be considered as an internal mixing project. 
- While it is likely a goal of industrial research, investigate if an accurate processingstructure-property relationship can be found for these materials. If so, one could presumably decouple the effects of material morphology from differing thermal and processing histories required to generate those structures.

- An unquestionably important role of industrial polymer scientists is to create optimized formulations depending on manufacturers' and customers' desired end applications. In this case, we know that PLA is much less chemically resistant and stable than polycarbonate. Therefore, while it may prove challenging in an academic setting, one may be interested in the effects of compounding in any number of (for example) thermal, hydrolytic, or even photo-oxidative stabilizing agents in an effort to enhance the PLA stability. Given the normal sub-percentage additions of these additives, this would almost certainly require use of the internal mixer for making precise formulations as metering capability with the current extruder and control systems is not sufficient.

- In the past few years, many publications have been written attempting to incorporate graphene and graphite sheets into composite materials. Authors often employ preprocessing steps on the inorganic additives; it has become conventional knowledge that polymer processing typically does not provide adequate energy input to fully exfoliate these materials. One could then attempt a study which links processing of a composite with subsequent analysis of the degree of dispersion of the graphene phase, and then to correlate the graphene structure with changes in physical and/or barrier properties. This could be especially interesting if performed in a multi-phase polymer blend. 


\section{REFERENCES}

${ }^{1}$ Wolf, O., Crank, M., Patel, M., Marscheider-Weidemann, F., Schleich, J., Hüsing, B., Angerer, G. Techno-economic Feasibility of Large Scale Production of Bio-Based Polymers in Europe. Technical Report EUR 22013 EN (2005).

${ }^{2} \mathrm{Yu}, \mathrm{L} .$, Dean, K., Li, L. Polymer blends and composites from renewable resources. Progress in Polymer Science 31 (2006) 576-602.

${ }^{3}$ Grunert, M., Winter, W.T . Nanocomposites of Cellulose Acetate Butyrate Reinforced with Cellulose Nanocrystals. Journal of Polymers and the Environment 10 (2002) 27-30.

${ }^{4}$ European Bioplastics (November 6, 2009). Bio-based plastics: New study forecasts enormous potential. Press Release. www.epnoe.eu

${ }^{5}$ European Bioplastics (October 2012). Policies for Bioplastics: A framework for sustainable industrial growth in the EU. Policy Paper. http://en.european-bioplastics.org/wpcontent/uploads/2012/publications/Policies_bioplastics2012.pdf

${ }^{6}$ Carole, T.M., Pellegrino, J., Paster, M.D. Opportunities in the Industrial Biobased Products Industry. Applied Biochemistry and Biotechnology 113-116 (2004) 871-885.

${ }^{7}$ Rasal, R.M., Janorkar, A.M., Hirt, D.E. Poly(lactic acid) modifications. Progress in Polymer Science 35 (2008) 338-356.

${ }^{8}$ Balkcom, M., Welt, B., Berger, K.. Notes from the Packaging Laboratory: Polylactic Acid - An Exciting New Packaging Material. University of Florida IFAS Extension publication ABE 339 (2002) 1-5.

${ }^{9}$ Yoon, J.T., Jeong, Y.G, Lee, S.C., Min, B.G. Influences of poly(lactic acid)-grafted carbon nanotube on thermal, mechanical and electrical properties of poly(lactic acid). Polymers for Advanced Technologies 20 (2009) 631-638.

${ }^{10}$ Braun, B., Dorgan, J.R., Knauss, D.M. Reactively Compatibilized Cellulosic Polylactide Microcomposites. Journal of Polymers and the Environment 14 (2006) 49-58.

${ }^{11}$ Witzke, D. Introduction to Properties, Engineering and Prospects of Polylactide Polymers. Ph.D dissertation. Michigan State University, 1997.

12 de Jong, S.J., Arias, E.R., Rijkers, D.T.S., van Nostrum, C.F., Kettenes-van den Bosch, J.J., Hennink, W. New Insights into the hydrolytic degradation of poly(lactic acid): participation of the alcohol terminus. Polymer 42 (2001) 2795-2802. 
${ }^{13} \mathrm{http}: / /$ www.natureworksllc.com/ /media/Technical_Resources/Technical_Data_Sheets/Technic alDataSheet_2003D_FFP-FSW_pdf.pdf

${ }^{14}$ Kawamoto, N., Sakai, A., Horikoshi, T., Urushihara, T., Tobita, El. Physical and Mechanical Properties of Poly(L-lactic acid) Nucleated by Dibenzoylhydrazide Compound. Journal of Applied Polymer Science 103 (2007) 244-250.

${ }^{15} \mathrm{Yu}, \mathrm{L} ., \mathrm{Liu}, \mathrm{H} ., \mathrm{Xie}, \mathrm{F} ., \mathrm{Li}, \mathrm{X}$. Effect of Annealing and Orientation on Microstructures and Mechanical Properties of Polylactic Acid. Polymer Engineering and Science 48 (2008) 634-641. ${ }^{16}$ Maharana, T., Mohanty, B., Negi, Y.S. Melt-Solid polycondensation of lactic acid and its biodegradibility. Progress in Polymer Science 34 (2009) 99-124.

${ }^{17}$ Renouf-Glauser, A.C., Rose, J., Farrar, D.F., Cameron, R.E. The effect of crystallinity on the deformation mechanism and bulk mechanical properties of PLLA. Biomaterials 26 (2005) 57715782.

${ }^{18} \mathrm{Kim}$, M.S., Kim, J.C., Kim, Y.H. Effects of take-up speed on the structure and properties of melt-spun poly(L-lactic acid) fibers. Polymers for Advanced Technologies 19 (2008) 748-755.

${ }^{19}$ Ghosh, S., Viana, J.C., Reis, R.L., Mano, J.F. Oriented morphology and enhanced mechanical properties of poly(L-lactic acid) from shear controlled orientation in injection molding.

Materials Science and Engineering A 490 (2008) 81-89.

${ }^{20}$ Hirata, M., Kimura, Y. et al. Thermomechanical properties of stereoblock poly(lactic acid)s with different PLLA/PDLA block compositions. Polymer 49 (2008) 2656-2661.

${ }^{21}$ Kolstad, J. Crystallization Kinetics of Poly(L-lactide-co-meso-lactide). Journal of Applied Polymer Science 62 (1996) 1079-1091.

${ }^{22}$ Ning, W., Xingxiang, Z., Jiugao, Y., Jianming, F. Study of the Properties of Plasticised Poly(Lactic Acid) with Poly(1,3-Butylene Adipate). Polymers and Polymer Composites 16 (2008) 297-604.

${ }^{23}$ Ali, F., Chang, Y.W., Kang, S.C., Yoon, J.Y. Thermal, mechanical and rheological properties of poly(lactic acid)/epoxidized soybean oil blends. Polymer Bulletin 62 (2009) 91-98.

${ }^{24}$ Xu, Y.Q., Qu, J.P. Mechanical and Rheological Properties of Epoxidized Soybean Oil Plasticized Poly(lactic acid). Journal of Applied Polymer Science 112 (2009) 3185-3191. 
${ }^{25}$ Okamoto, K., Ichikawa, T., Yokohara, T., Yamaguchi, M. Miscibility, mechanical and thermal properties of poly(lactic acid)/polyester-diol blends. European Polymer Journal 45 (2009) 23042312.

${ }^{26} \mathrm{Yu}, \mathrm{T} .$, Ren, J., Gu, S., Yang, M. Synthesis and Characterization of poly(lactic acid) and aliphatic polycarbonate copolymers. Polymer International 58 (2009) 1058-1064.

${ }^{27} \mathrm{Gu}$, S., Yang, M., Yu, T., Ren, T., Ren, J. Synthesis and characterization of biodegradable lactic acid-based polymers by chain extension. Polymer International 57 (2008) 982-986.

${ }^{28} \mathrm{Li}$, Y., Shimizu, H. Improvement in toughness of poly (L-lactide) (PLLA) through reactive blending with acrylonitrile-butadiene-styrene (ABS): Morphology and properties. European Polymer Journal 45 (2009) 738-746.

${ }^{29}$ Ko, S.W., Hong, M.K., Park, B.J., Gupta, R.K., Choi, H.J., Bhattacharya, S.N. Morphological and rheological characterization of multi-walled carbon nanotube/PLA/PBAT blend nanocomposites. Polymer Bulletin 63 (2009) 125-134.

${ }^{30}$ Xiao, H., Lu, W., Yeh, J.T. Crystallization Behavior of Fully Biodegradable Poly(Lactic Acid)/Poly(Butylene Adipate-co-Terephthalate) Blends. Journal of Applied Polymer Science 112 (2009) 3754-3763.

${ }^{31}$ Yokohara, T., Yamaguchi, M. Structure and properties for biomass-based polyester blends of PLA and PBS. European Polymer Journal 44 (2008) 677-685.

${ }^{32}$ Liu, F., Zhao, Z., Yang, J., Wei, J., Li, S. Enzyme-catalyzed degradation of poly(Llactide)/poly(E-caprolactone) diblock, triblock and four-armed copolymers. Polymer Degradation and Stability 94 (2009) 227-233.

${ }^{33}$ Takayama, T., Todo, M., Tsuji, H. Effect of annealing on the mechanical properties of PLA/PCL and PLA/PCL/LTI polymer blends. Journal of the Mechanical Behavior of Biomedical Materials (2010) doi:10.106/j.jmbbm.2010.10.003

${ }^{34}$ Chen, H., Pyda, M., Cebe, P. Non-isothermal crystallization of PET/PLA blends. Thermochimica Acta 492 (2009) 61-66.

${ }^{35}$ Cohen, S., Yoshioka, T., Lucarelli, M., Hwang, L.H., Langer, R. Controlled Delivery Systems for Proteins Based on Poly(Lactic/Glycolic Acid) Microspheres. Pharmaceutical Research 8 (1991) 713-720. 
${ }^{36}$ Dunne, M., Corrigan, O.I., Ramtoola, Z.. Influence of particle size and dissolution conditions on the degradation of polylactide-co-glycolide particles. Biomaterials 21 (2000) 1659-1668.

${ }^{37}$ Fu, B.X., Hsiao, B.S., Chen, G., Zhou, J., Koyfman, I., Jamiolkowski, D.D., Dormier, E. Structure and property studies of bioabsorbable poly(glycolide-co-lactide) fiber during processing and in vitro degradation. Polymer 43 (2002) 5527-5534.

${ }^{38} \mathrm{Li}$, S. Hydrolytic Degradation Characteristics of Aliphatic Polyesters Derived from Lactic and Glycolic Acids. Journal of Biomedical Material Research (Applied Biomaterials) 48 (1999) 342353.

${ }^{39}$ Wang, Z.G., Wang, X., Hsiao, B.S., Andjelić, S., Jamiolkowski, D.D., McDivitt, J., Fischer, J., Zhou, J., Han, C.C. Time-resolved isothermal crystallization of absorbable PGA-co-PLA copolymer by synchrotron small-angle X-ray scattering and wide-angle X-ray diffraction. Polymer 42 (2001) 8965-8973.

${ }^{40}$ Harper, C. (ed.) Modern Plastics Handbook. McGraw-Hill: 2000.

${ }^{41}$ Watanabe, M., Matsuo, Y., Matsushita, T., Inomata, H., Miyake, T., Hironaka, K. Chemical Recycling of Polycarbonate in high pressure high temperature steam at $573 \mathrm{~K}$. Polymer Degradation and Stability 94 (2009) 2157-2162.

${ }^{42}$ Ishihara, J., Kuyama, H., Ozeki, E., Ishitoku, T., Tanaka, M., Sakamoto, N. Crosslinked polycarbonate and polylactic acid composition containing the same. European Patent Application EP 0896013 A1, 1999.

${ }^{43}$ Kanamori, T., Urayama, H., Takakuwa, K., Nakamura, M., Teraya, Y., Shimotsuma, S. Biodegradable polyester/polyester carbonate resin composition. European Patent Application EP 0990678 A2, 2000.

${ }^{44}$ Aoki, Y., Noritaka, S. Resin composition. European Patent Application EP 2096142 A1, 2009.

${ }^{45}$ Lee, H.T., Ha, D.H., Jung, C.D., Kwon, Y.C., Cho, J.K., Chung, Y.M., Lee, B.C. Polylactic acid/polycarbonate resin composition and molded product made using the same. European Patent Application EP 2199342 A2, 2010.

${ }^{46}$ Chung, J.Y., Mason, J.P. Impact-Modified Polycarbonate-Polylactic Acid Composition. World Patent Application WO 2010/019207 A2, 2010. 
${ }^{47}$ Warth, H., Chen, S., Wang, Y., Wang, Y., Li, H. A Blend of Aromatic Polycarbonate and Polylactic Acid, the Method for Preparing the Same Und (sic) the Use Thereof. World Patent Application WO2010/028785 A1, 2010.

${ }^{48}$ Chung, Y.M., Jung, C.D., Lee, Y.J., Pack, J.W., Kwon, Y.C., Lee, H.T., Cho, J.K.

Environmentally Friendly Polylactic Acid Resin Composition. US Patent 8232343, issued July 31, 2012.

${ }^{49}$ Chung, Y.M., Jung, C.D. Polylactic Acid Resin Composition. EU Patent 2133392, issued February 23, 2011.

${ }^{50}$ Lee, J.B., Lee, Y.K., Choi, G.D., Na, S.W., Park, T.S., Kim, W.N. Compatibilizing effects for improving mechanical properties of biodegradable poly(lactic acid) and polycarbonate blends. Polymer Degradation and Stability 96 (2011) 553-560.

${ }^{51}$ Ikehara, T., Nishikawa, Y., Nishi, T. Evidence for the formation of interpenetrated spherulites in poly(butylenes succinate-co-butylene carbonate)/poly(L-lactic acid) blends investigated by atomic force microscopy. Polymer 44 (2003) 6657-6661.

${ }^{52}$ Hung, T.F., Lai, M.T., Wang, Y., Chiao, S.M. Morphology and Properties of Polylactide and Polycarbonate Green Blends. ANTEC (2011) 291-294.

${ }^{53}$ Zhou, Y., Wu, G.L., Zhuo, R.X., Liu, Z.L. Synthesis and properties of novel aliphatic poly(carbonate-ester)s. European Polymer Journal 45 (2009) 1868-1872.

${ }^{54}$ Bruice, P. Organic Chemistry. $3^{\text {rd }}$ ed. Prentice Hall: Upper Saddle River, NJ, 2001. ${ }^{55}$ Nabar, S., Kale, D.D. Rheology and Transesterification between Polycarbonate and Polyester. Journal of Applied Polymer Science 104 (2007) 2039-2047.

${ }^{56}$ de Jong, S. J., Arias, E.R., Rijkers, D.T.S., van Nostrum, C.F., Kettenes-van den Bosch, J.J., Hennink, W. et al. New Insights into the hydrolytic degradation of poly(lactic acid): participation of the alcohol terminus. Polymer 42 (2001) 2795-2802.

${ }^{57}$ Makino, K., Arakawa, M., Kondo, T. Preparation and in Vitro Degradation properties of Polylactide Microcapsules. Chemical and Pharmaceutical Bulletin 33 (1985) 1195-1201.

${ }^{58}$ Makino, K., Ohshima, H., Kondo, T. Mechanism of hydrolytic degradation of poly(L-lactide) microcapsules: effects of $\mathrm{pH}$, ionic strength and buffer concentration. Journal of Microencapsulation 3 (1986) 203-212. 
${ }^{59}$ Mohd-Adnan, A.F., Nishida, H., Shirai, Y. Evaluation of kinetics parameters for poly(L-lactic acid) hydrolysis under high pressure steam. Polymer Degradation and Stability 93 (2008) 10531058.

${ }^{60}$ von Burkersroda, F., Schedl, L., Göpferich, A. Why degradable polymers undergo surface erosion or bulk erosion. Biomaterials 23 (2002) 4221-4231.

61 Zhang, X., Espiritu, M., Bilyk, A., Kurniawan, L. Morphological behaviour of poly(lactic acid) during hydrolytic degradation. Polymer Degradation and Stability 93 (2008) 1964-1970.

62 Zhou, Q.; Xanthos, M. Nanoclay and crystallinity effects on the hydrolytic degradation of polylactides. Polymer Degradation and Stability 93 (2008) 1450-1459.

${ }^{63}$ Harris, A.M., Lee, E.C. Heat and Humidity Performance of Injection Molded PLA for durable applications. Journal of Applied Polymer Science 115 (2010) 1380-1389.

64 Tsuji, H., Saeki, T., Tsukegi, T., Daimon, H., Fujie, K. Comparative study on hydrolytic degradation and monomer recovery of poly(L-lactic acid) in the solid and in the melt. Polymer Degradation and Stability 93 (2008) 1956-1963.

65 Tsuji, H., Ikarashi, K., Fukuda, N. Poly(L-lactide): XII. Formation, growth, and morphology of crystalline residues as extended-chain crystallites through hydrolysis of poly(L-lactide) films in phosphate-buffered solution. Polymer Degradation and Stability 84 (2004) 515-523.

66 Tsuji, H. In vitro hydrolysis of blends from enantiomeric poly(lactide)s. Part 4: well-homocrystallized blend and nonblended films. Biomaterials 24 (2003) 537-547.

${ }^{67}$ Okamoto, H., Nakano, M., Ouchi, M., Usuki, A., Kageyama, Y. Improvement of Crystallization and Mechanical Properties of PLA by Means of Clay Nanocomposite. Materials Research Society Symposium Proceedings 791 (2004) 399-404.

${ }^{68}$ Rhim, J.W., Hong, S.I., Ha, C.S. Tensile, water vapor barrier and antimicrobial properties of PLA/nanoclay composite films. LWT - Food Science and Technology 42 (2009) 612-617.

${ }^{69}$ Tagaya, H., Katoh, K., Kadokawa, J., Chiba, K. Decomposition of polycarbonate in subcritical and supercritical water. Polymer Degradation and Stability 64 (1999) 289-292.

70 Zinbo, M., Golovoy, A. Determination of the Long-Term Hydrolytic Stability of Polycarbonate Engineering Resins. Polymer Engineering and Science 32 (1992) 786-791.

${ }^{71}$ Viljanmaa, M., Södergård, A., Törmälä, P. Lactic acid based polymers as hot melt adhesives for packaging applications. International Journal of Adhesion \& Adhesives 22 (2002) 219-226. 
${ }^{72}$ Broz, M.E., VanderHart, D.L., Washburn, N.R. Structure and mechanical properties of poly(D,L-lactic acid)/poly(E-caprolactone) blends. Biomaterials 24 (2003) 4181-4190.

${ }^{73}$ Leclair, A., Favis, B.D. The role of interfacial contact in immiscible binary polymer blends and its influence on mechanical properties. Polymer 37 (1996) 4723-4728.

${ }^{74}$ Zhang, G., Zhang, J., Wang, S., Shen, D. Miscibility and Phase Structure of Binary Blends of Polylactide and Poly(methyl methacrylate). Journal of Polymer Science: Part B: Polymer Physics 40 (2003) 23-30.

${ }^{75}$ Chang, J.H., An, Y.U., Sur, G.S. Poly(lactic acid) Nanocomposites with Various Organoclays. I. Thermomechanical Properties, Morphology, and Gas Permeability. Journal of Polymer Science: Part B: Polymer Physics 41 (2003) 94-103.

${ }^{76}$ Li, B., Dong, F.X., Wang, X.L., Yang, J., Wang, D.Y., Wang, Y.Z. Organically modified rectorite toughened poly(lactic acid): Nanostructures, crystallization and mechanical properties. European Polymer Journal 45 (2009) 2996-3003.

${ }^{77}$ Huda, M.S., Drzal, L.T., Mohanty, A.K., Misra, M. Effect of fiber surface-treatments on the properties of laminated biocomposites from poly(lactic acid) (PLA) and kenaf fibers. Composite Science and Technology 68 (2008) 424-432.

${ }^{78}$ Huda, M.S., Drzal, L.T., Mohanty, A.K., Misra, M. Effect of chemical modifications of the pineapple leaf fiber surfaces on the interfacial and mechanical properties of laminated biocomposites. Composite Interfaces 15 (2008) 169-191.

${ }^{79}$ Narimissa, E., Gupta, R., Bhaskaran, M., Sriram, S. Influence of nano-graphite platelet concentration on onset of crystalline degradation in polylactide composites. Polymer Degradation and Stability 97 (2012) 829-832.

${ }^{80}$ Avrami, M. Kinetics of Phase Change. I: General Theory. Journal of Chemical Physics 7 (1939) 1103-1112.

${ }^{81}$ Avrami, M. Kinetics of Phase Change. II: Transformation-Time Relations for Random Distribution of Nuclei. Journal of Chemical Physics 8 (1940) 212-224.

${ }^{82}$ Avrami, M. Kinetics of Phase Change. III: Granulation, Phase Change, and Microstructure. Journal of Chemical Physics 9 (1941) 177-184.

${ }^{83}$ Kumar, Anil; Gupta, Rakesh K.. Fundamentals of Polymer Engineering. $2^{\text {nd }}$ ed. Marcel Dekker, New York, 2003. 
${ }^{84}$ Sun, N.X., Liu, X.D., Lu, K. An explanation to the anomalous Avrami exponent. Scripta Materiala 34 (1996) 1201-1207.

${ }^{85}$ Pantani, R., De Santis, F., Sorrentino, A., De Maio, F., Titomanlio, G. Crystallization kinetics of virgin and processed poly(lactic acid). Polymer Degradation and Stability 95 (2010) 11481159.

${ }^{86}$ Cebe, P., Hong, S.D. Crystallization Behaviour of poly(ether-ether-ketone). Polymer 27 (1986) 1183-1192.

${ }^{87}$ Boon, J., Azcue, J.M. Crystallization Kinetics of Polymer-Diluent Mixtures. Influence of Benzophenone on the Spherulitic Growth Rate of Isotactic Polystyrene. Journal of Polymer Science 6 (1968) 885-894.

${ }^{88}$ Hoffman, J., Miller, R. Kinetics of crystallization from the melt and chain folding in polyethylene fractions revisited: theory and experiment. Polymer 38 (1997) 3151-3212.

${ }^{89}$ Al-Mulla, A., Al-Omairi, L., Mathew, J., Bhattacharya, S. Isothermal Crystallization Kinetics of Tricomponent Blends of Polycarbonate, Poly(Trimethylene Terephthalate) and Polybutylene Terephthalate. Polymers and Polymer Composites 18 (2010) 307-313.

${ }^{90}$ Yasuniwa, M., Tsubakihara, S., Sugimoto, Y., Nakafuku, C. Thermal Analysis of the DoubleMelting Behavior of Poly(L-lactic acid). Journal of Polymer Science: Part B: Polymer Physics 42 (2004) 25-32.

${ }^{91}$ Ginde, R.M., Gupta, R.K. In vitro chemical degradation of Poly(Glycolic Acid) Pellets and Fibers. Journal of Applied Polymer Science 33 (1987) 2411-2429.

92 ASTM D3826-98. Standard Practice for Determining Degradation End Point in Degradable Polyethylene and Polypropylene using a Tensile Test, 2013.

${ }^{93}$ Okada, A., Kawasumi, M., Usuki, A., Kojima, Y., Kurauchi, T., Kamigaito, O. Nylon 6-Clay Hybrid. Mat. Res. Soc. Symp. Proc. 171 (1990) 45-50.

${ }^{94}$ Lorenzo, A.T., Arnal, M.L., Albuerne, J., Müller, A.J. DSC isothermal polymer crystallization kinetics measurements and the use of the Avrami equation to fit the data: Guidelines to avoid common problems. Polymer Testing 26 (2007) 222-231.

${ }^{95}$ Wang, H., Qiu, Z. Crystallization behaviors of biodegradable poly(L-lactic acid)/graphene oxide nanocomposites from the amorphous state. Thermochimica Acta 526 (2011) 229-236. 
${ }^{96}$ Islam, M.S., Pickering, K.L., Foreman, N.J. Influence of accelerated ageing on the physicmechanical properties of alkali-treated industrial hemp fibre reinforced poly(lactic acid) (PLA) composites. Polymer Degradation and Stability 95 (2010) 59-65.

${ }^{97}$ Derkach, S. Rheology of emulsions. Advances in Colloid and Interface Science 151 (2009) 123.

${ }^{98}$ ASTM E799-03. Standard Practice for Determining Data Criteria and Processing for Liquid Drop Size Analysis, 2009.

${ }^{99}$ Crank, J. Mathematics of Diffusion, $2^{\text {nd }}$ ed. Oxford: Clarendon Press.

${ }^{100}$ Siparsky, G.L., Voorhees, K.J., Dorgan, J.R., Schilling, K. Water Transport in Polylactic Acid (PLA), PLA/Polycaprolactone Copolymers, and PLA/Polyethylene Glycol Blends. Journal of Environmental Polymer Degradation 5 (1997) 125-136.

${ }^{101}$ Moon, S.I., Extrand, C.W. Water Vapor Permeation Resistance of Polycarbonate at Various Temperatures. Ind. Eng. Chem. Res. 48 (2009) 8961-8965.

${ }^{102}$ Pogacnik, J. The Ductile to Brittle Transition in Polycarbonate. Ph.D. dissertation. Duke University, 2011. 


\section{Appendix A: Viscosity Curves for Solvent Degradation}

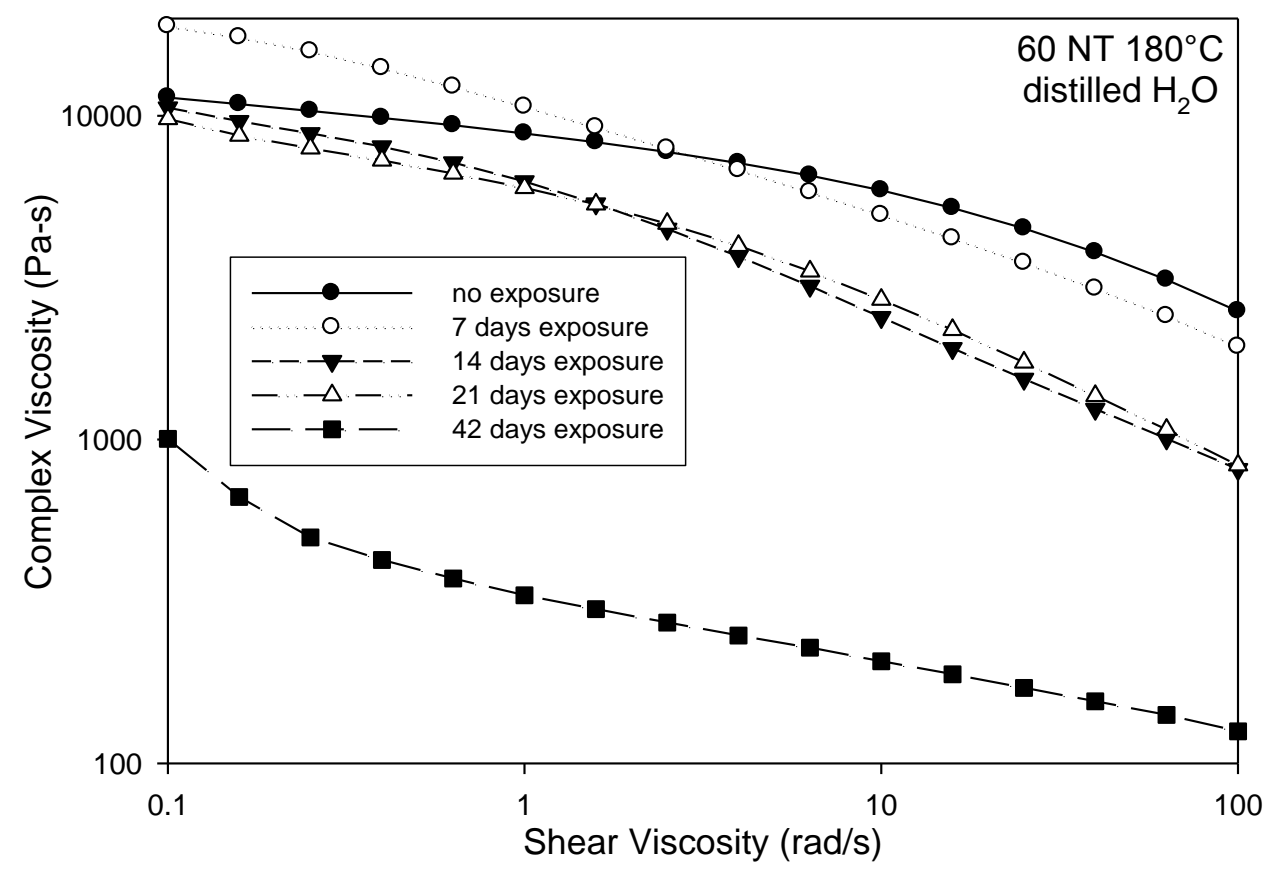

Figure A 1: Viscosity change of sample $60 \mathrm{NT}$ after distilled water degradation.

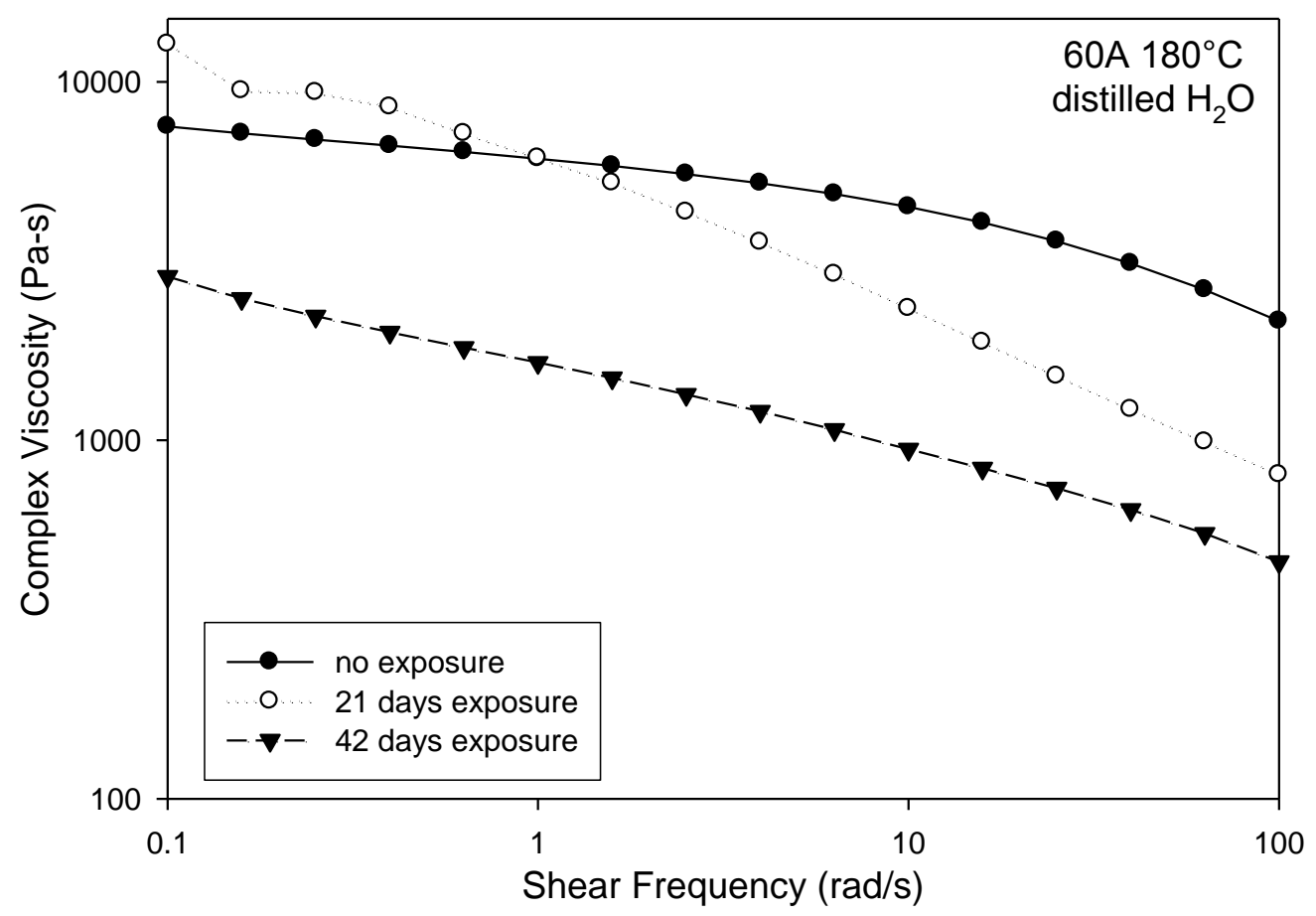

Figure A 2: Viscosity change of sample 60A after distilled water degradation. 


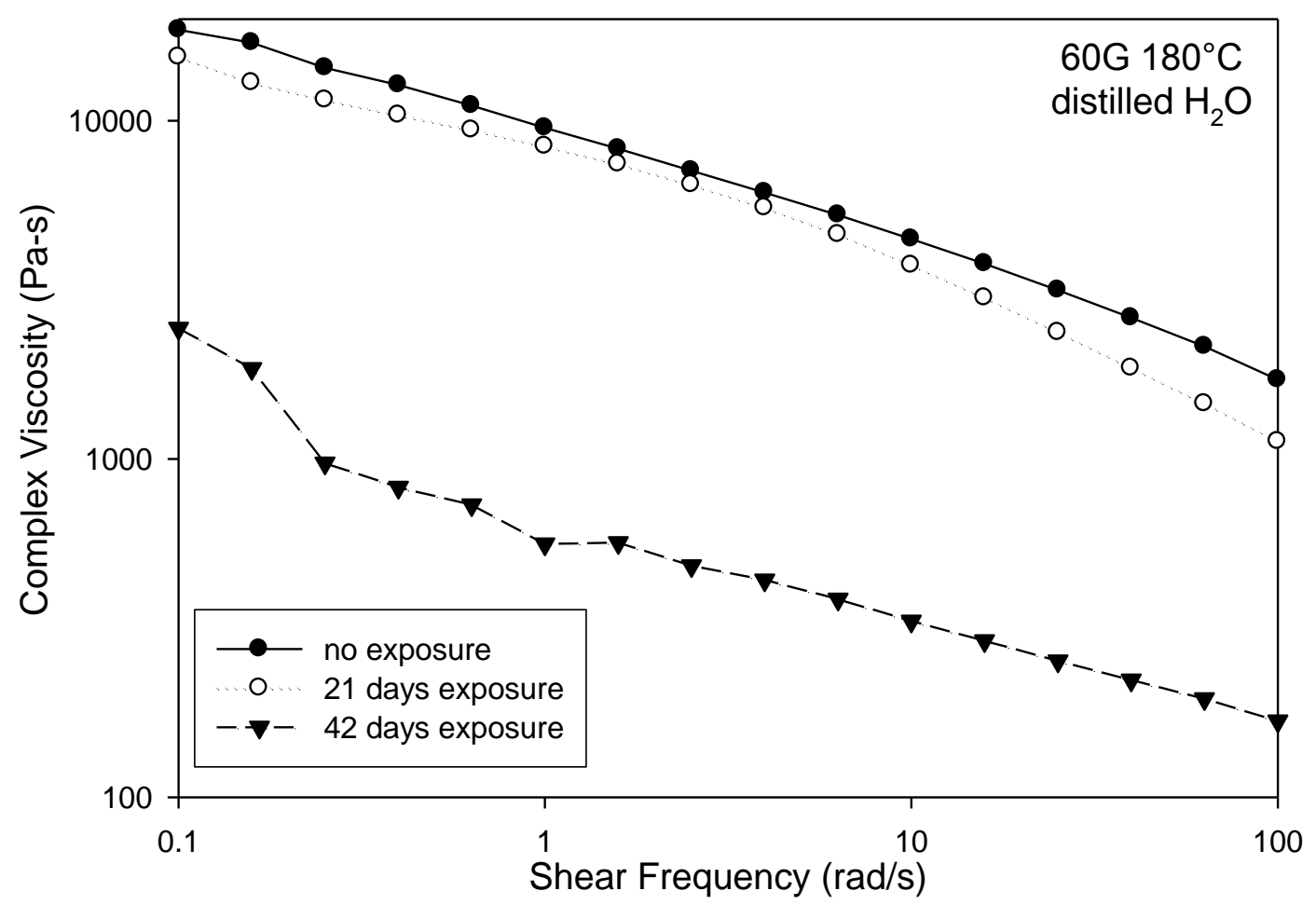

Figure A 3: Viscosity change of sample 60G after distilled water degradation.

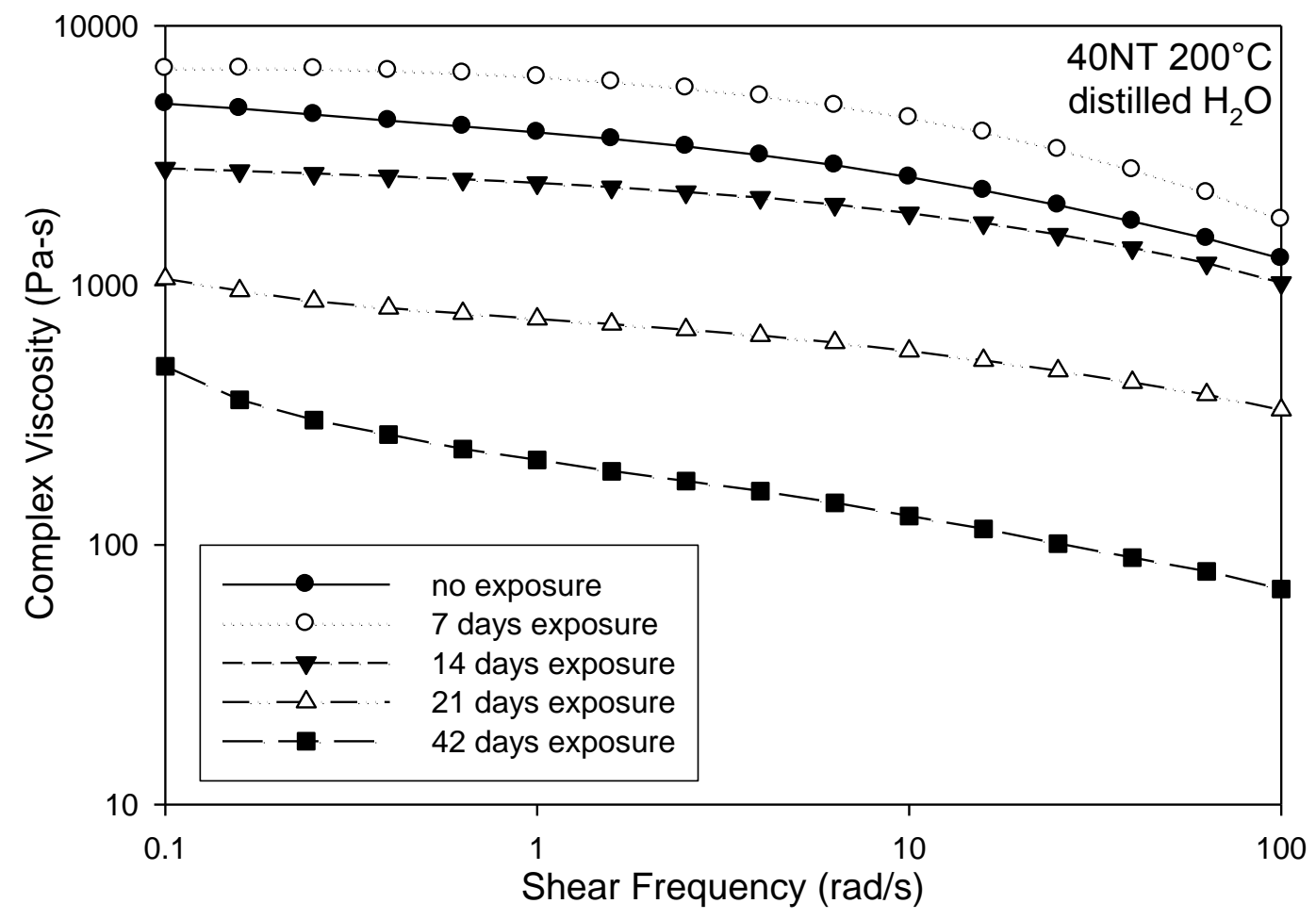

Figure A 4: Viscosity change of sample 40NT after distilled water degradation. 


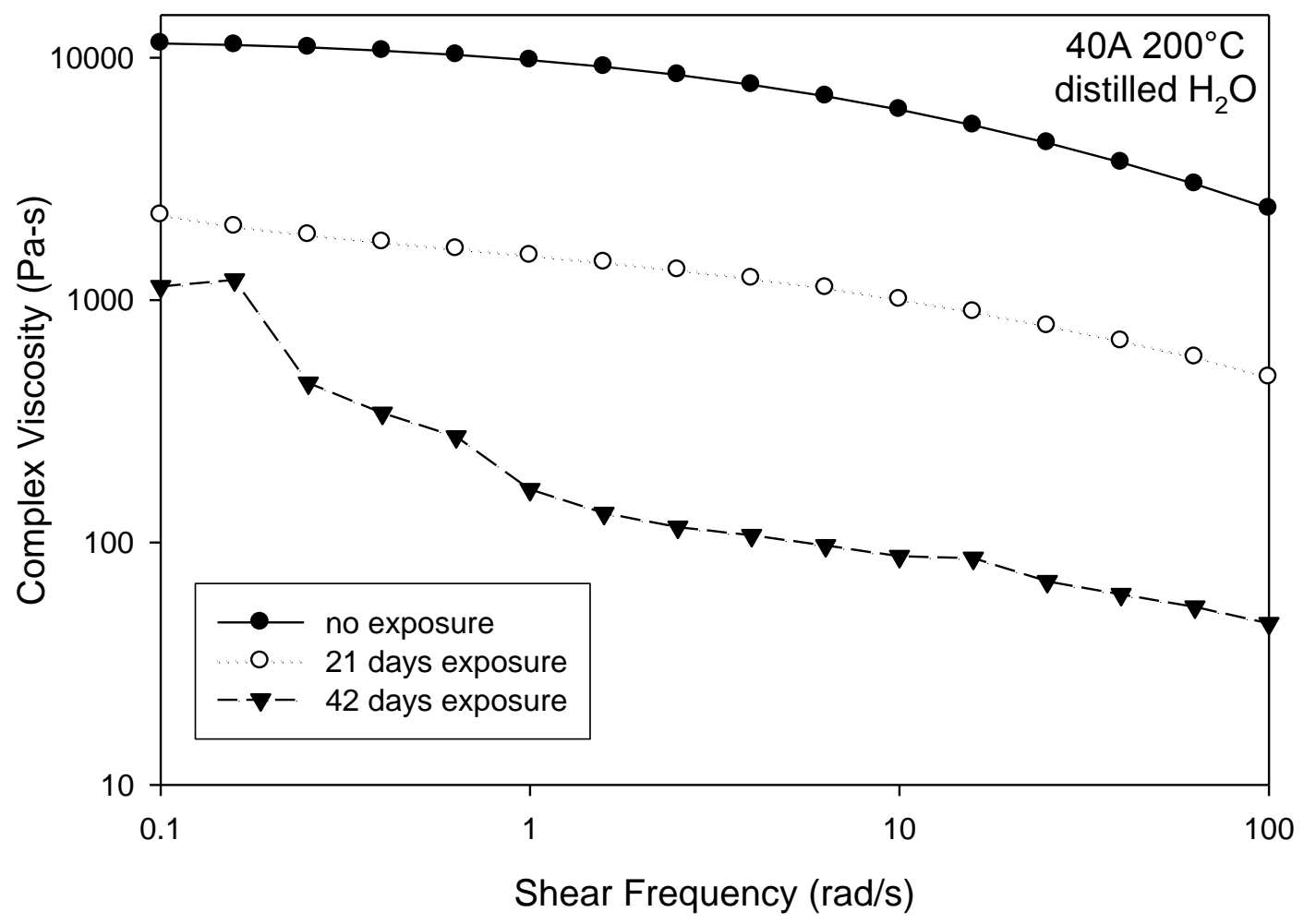

Figure A 5: Viscosity change of sample 40A after distilled water degradation.

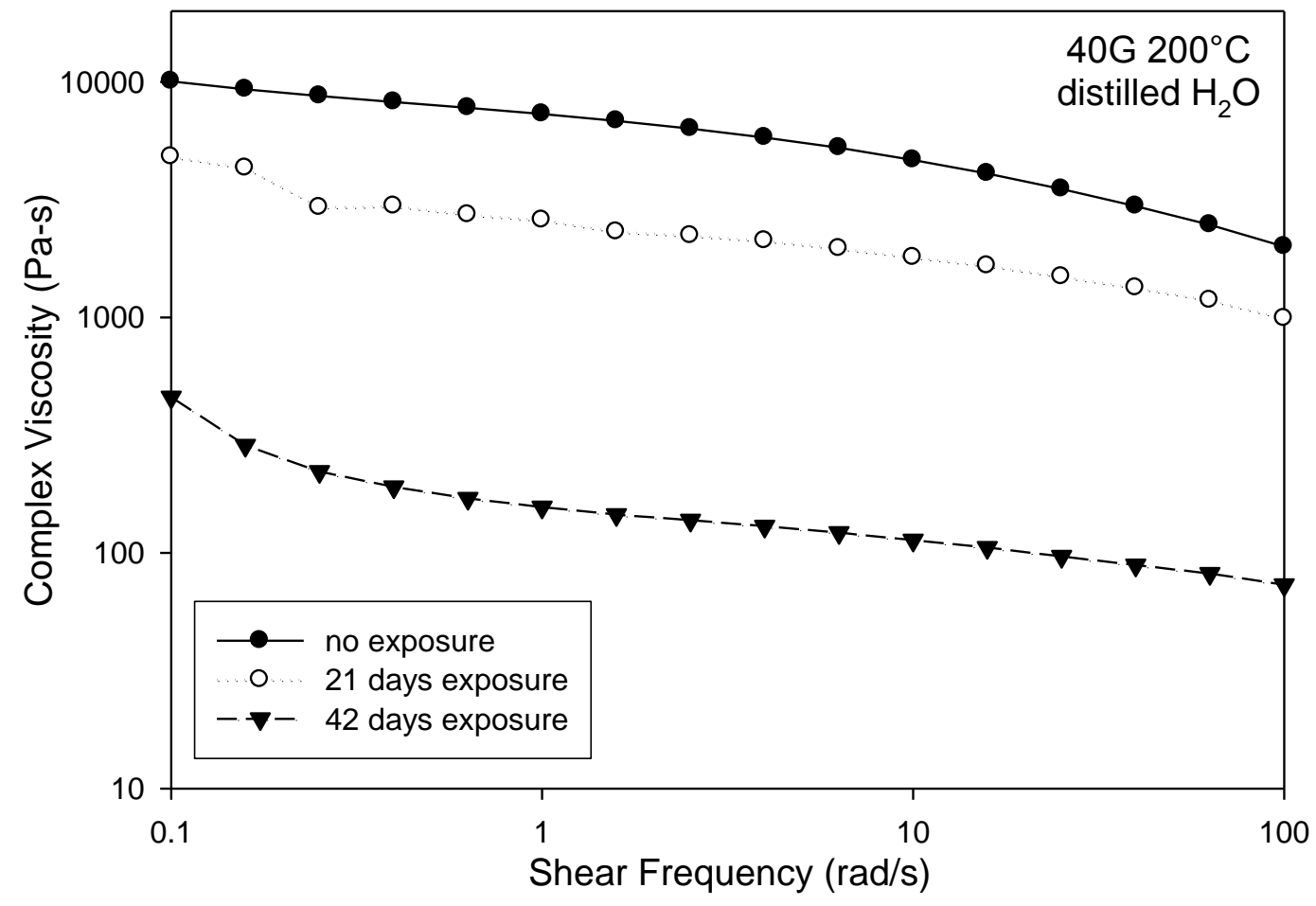

Figure A 6: Viscosity change of sample 40G after distilled water degradation. 


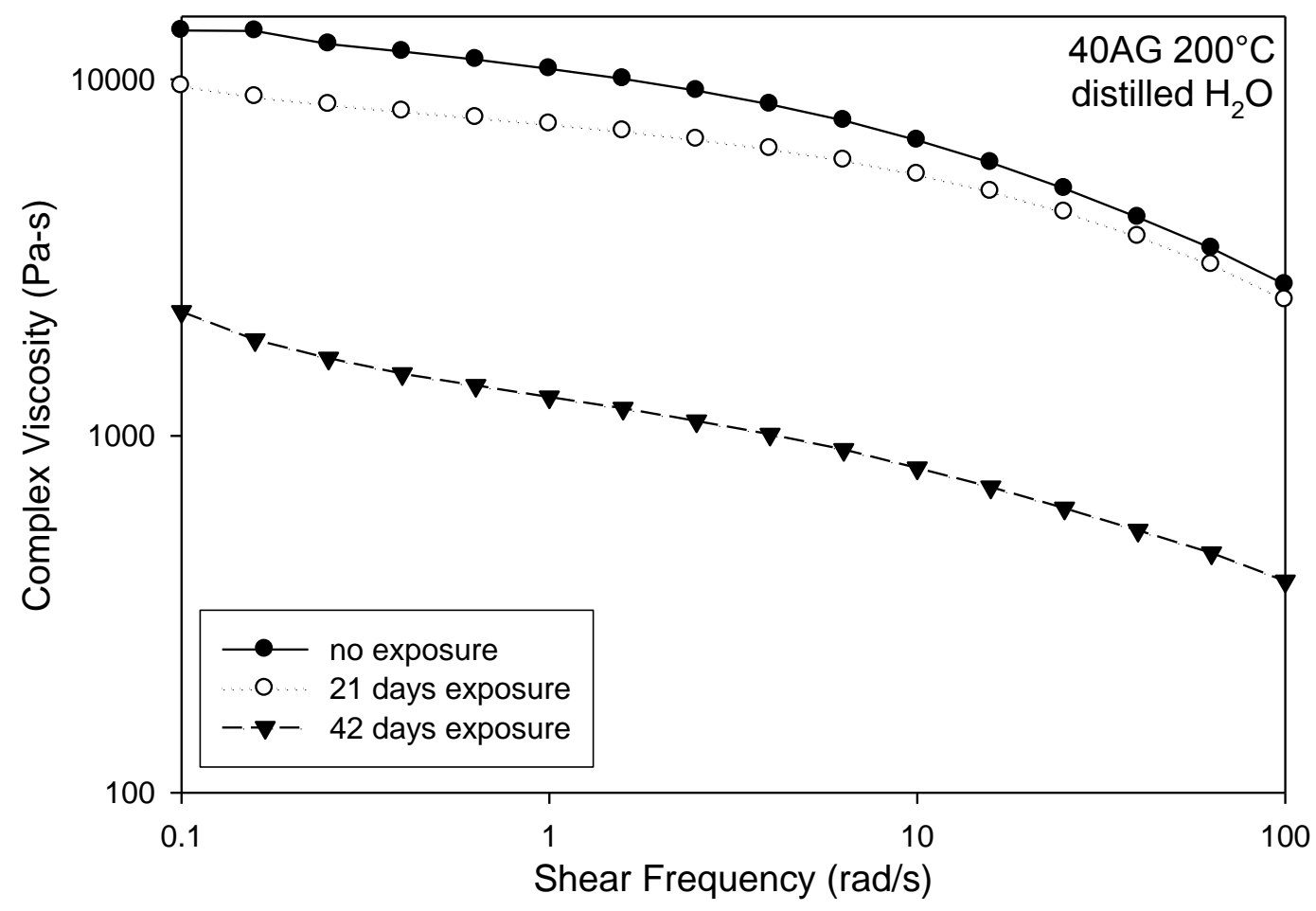

Figure A 7: Viscosity change of sample 40AG after distilled water degradation.

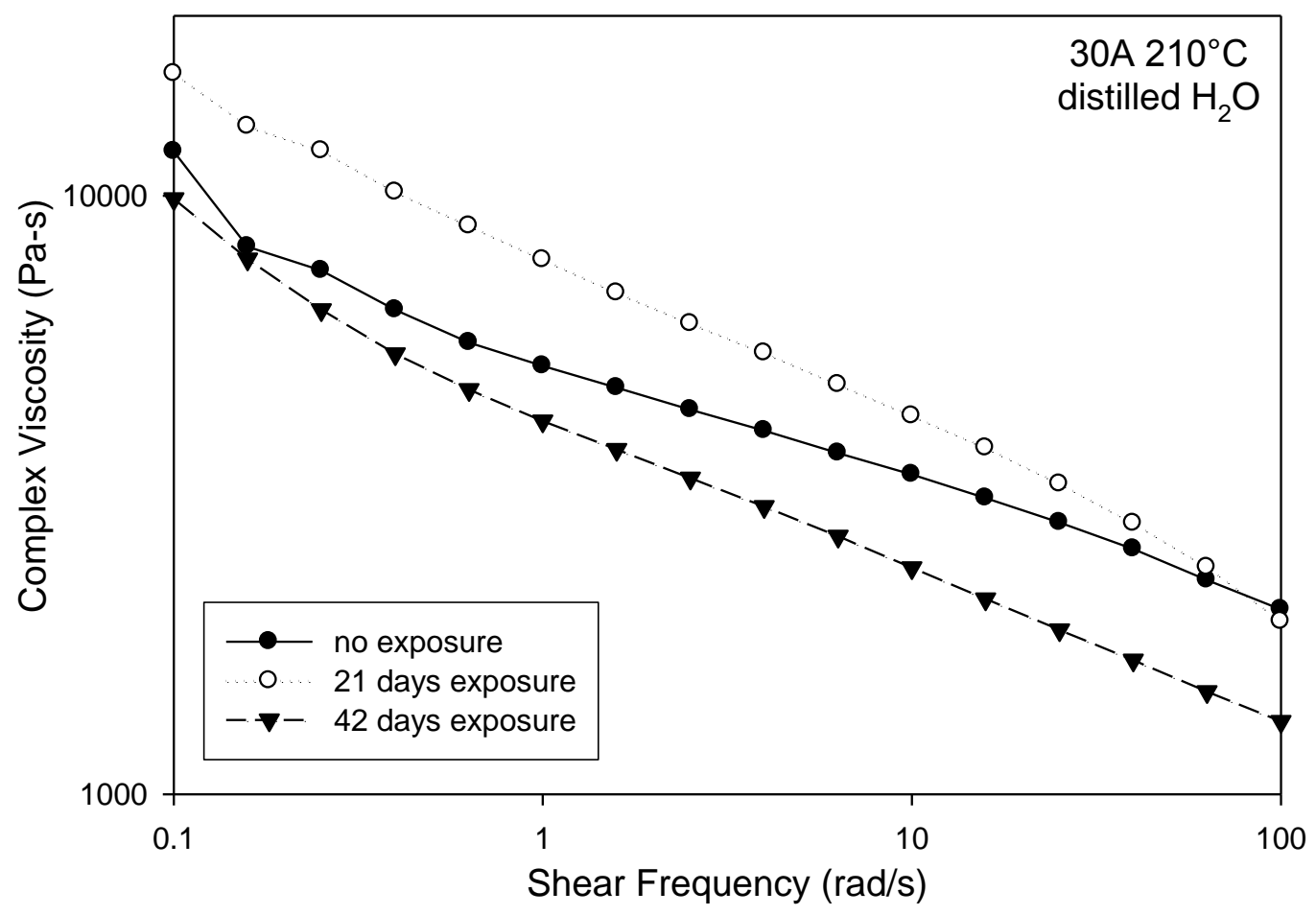

Figure A 8: Viscosity change of sample 30A after distilled water degradation. 


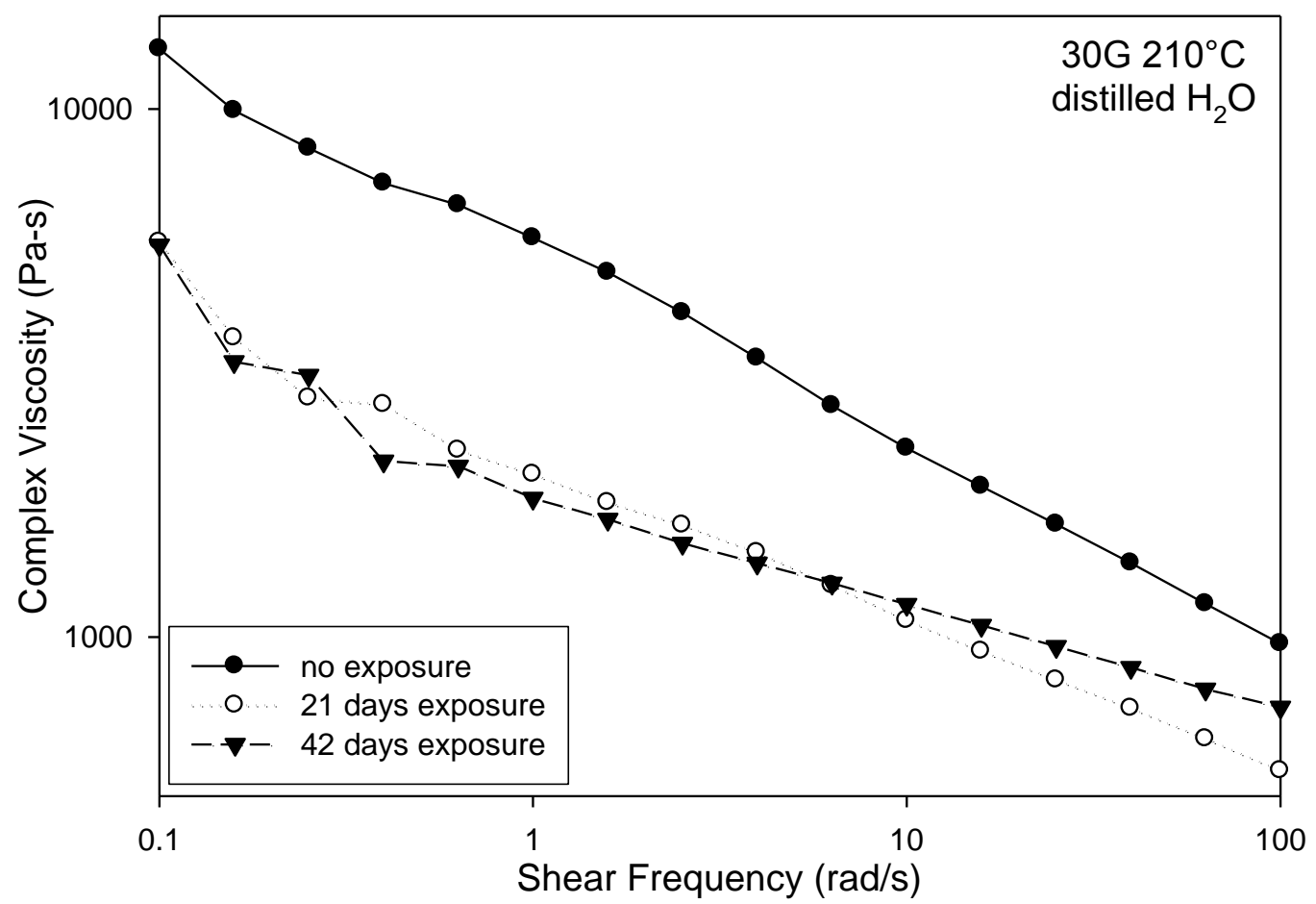

Figure A 9: Viscosity change of sample 30G after distilled water degradation.

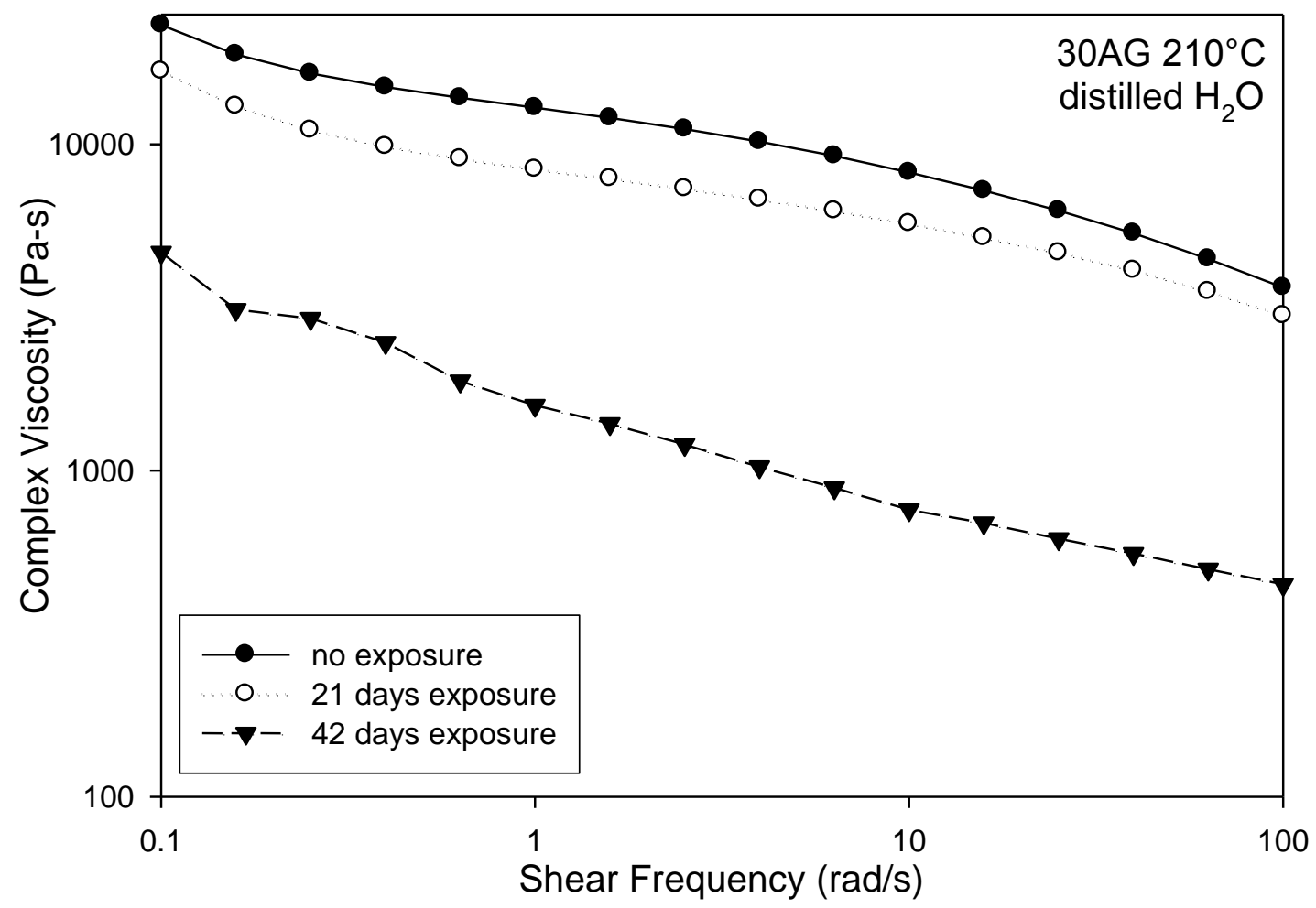

Figure A 10: Viscosity change of sample 30AG after distilled water degradation. 


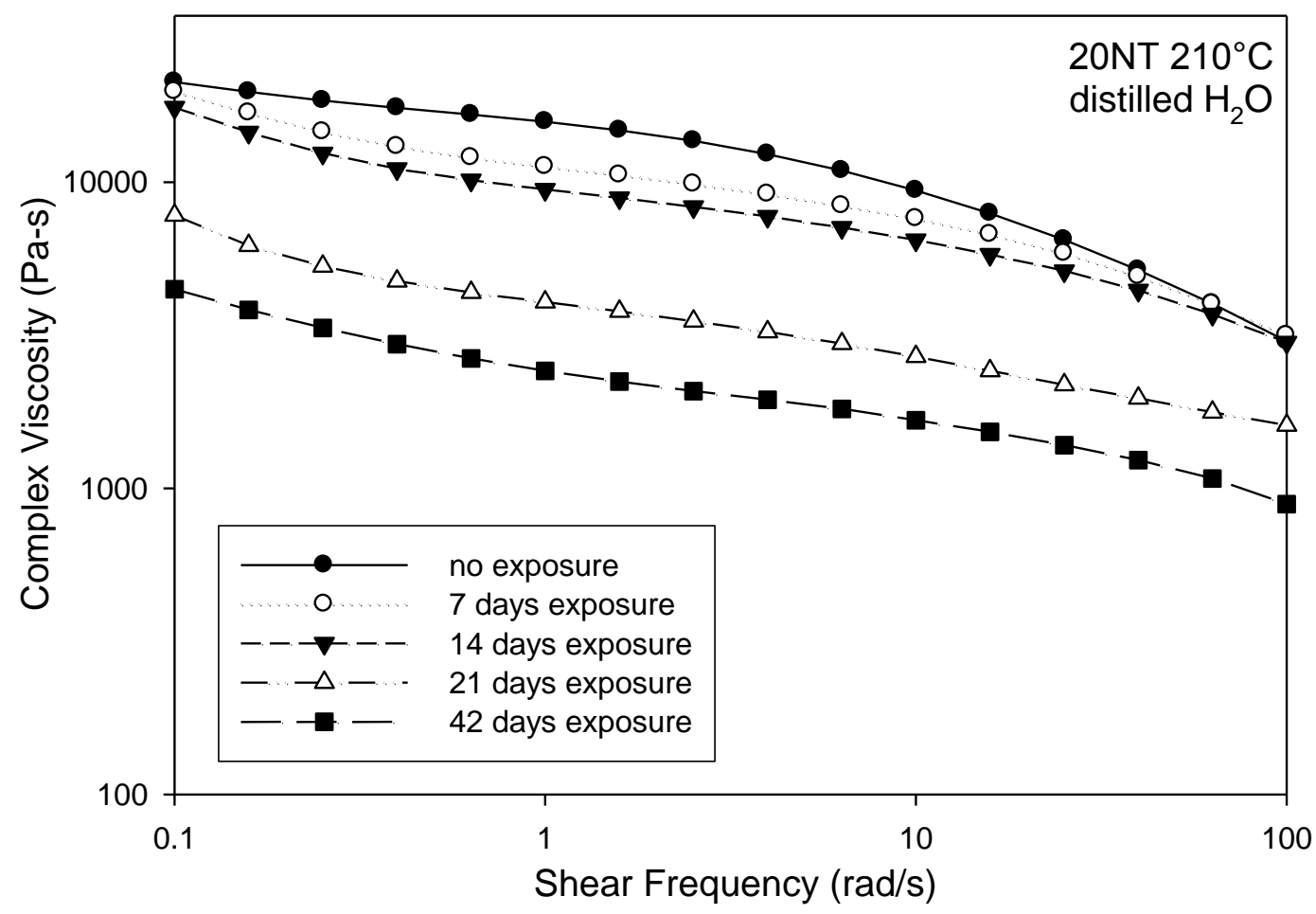

Figure A 11: Viscosity change of sample 20NT after distilled water degradation.

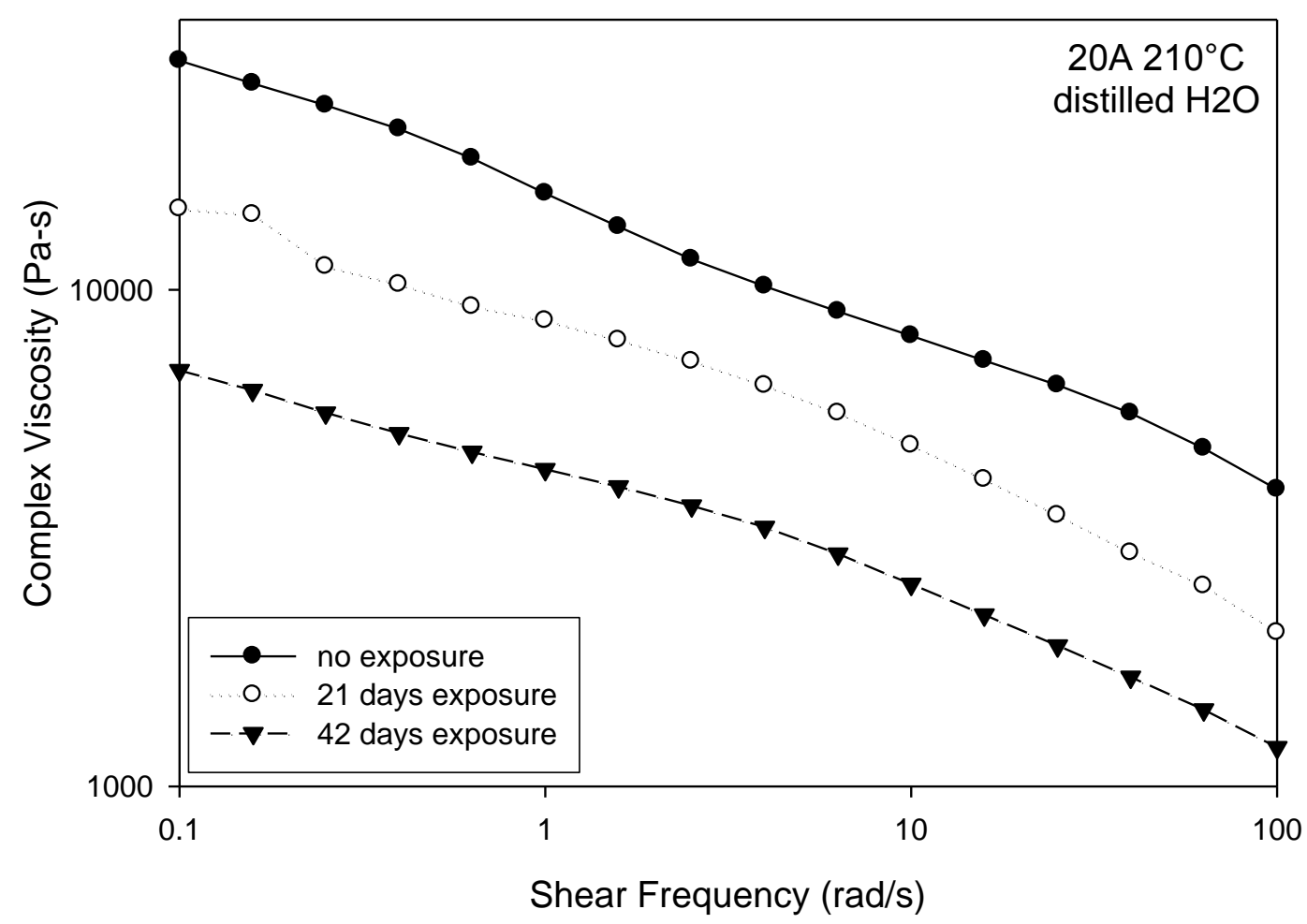

Figure A 12: Viscosity change of sample 20A after distilled water degradation. 


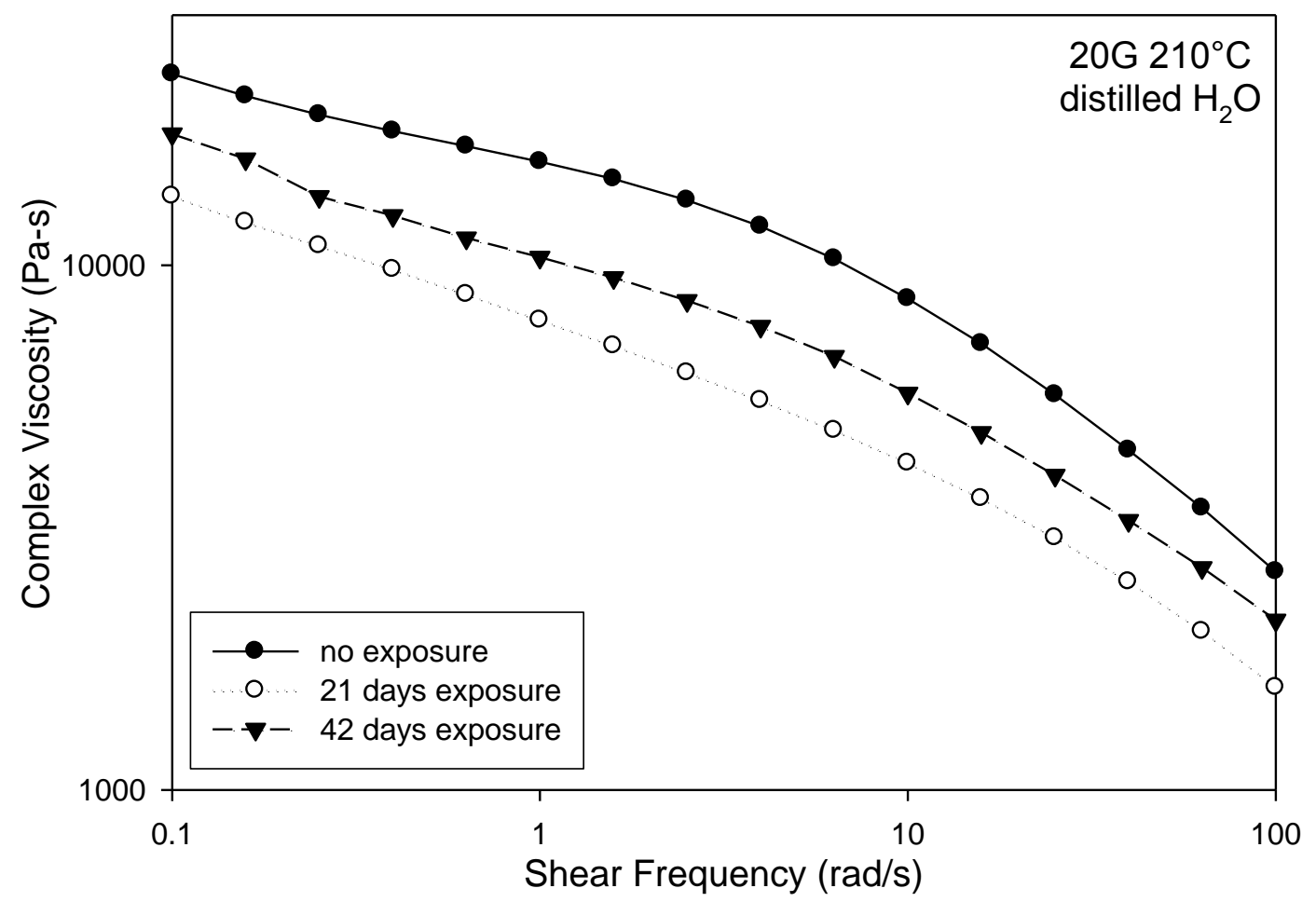

Figure A 13: Viscosity change of sample 20G after distilled water degradation.

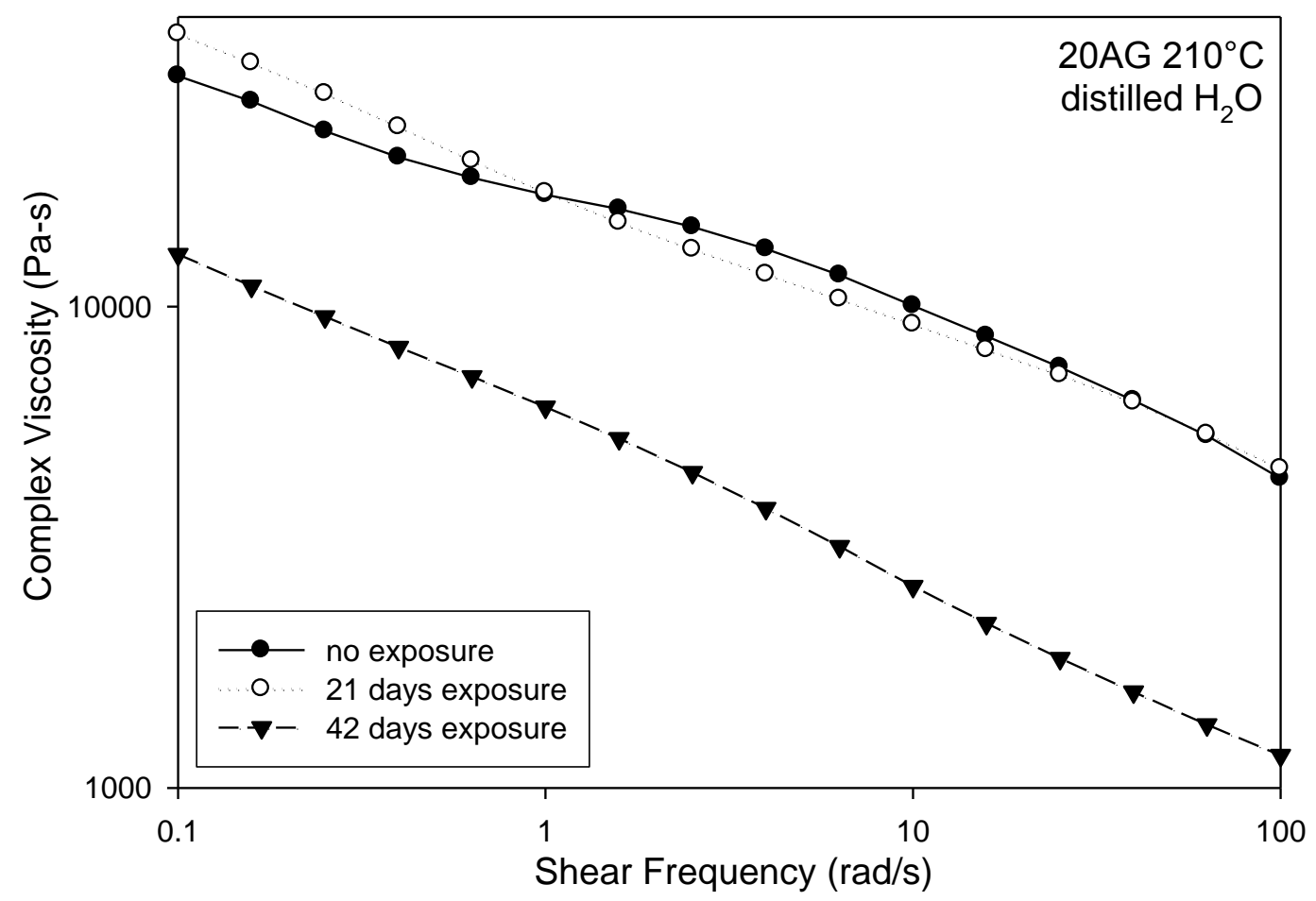

Figure A 14: Viscosity change of sample 20AG after distilled water degradation. 


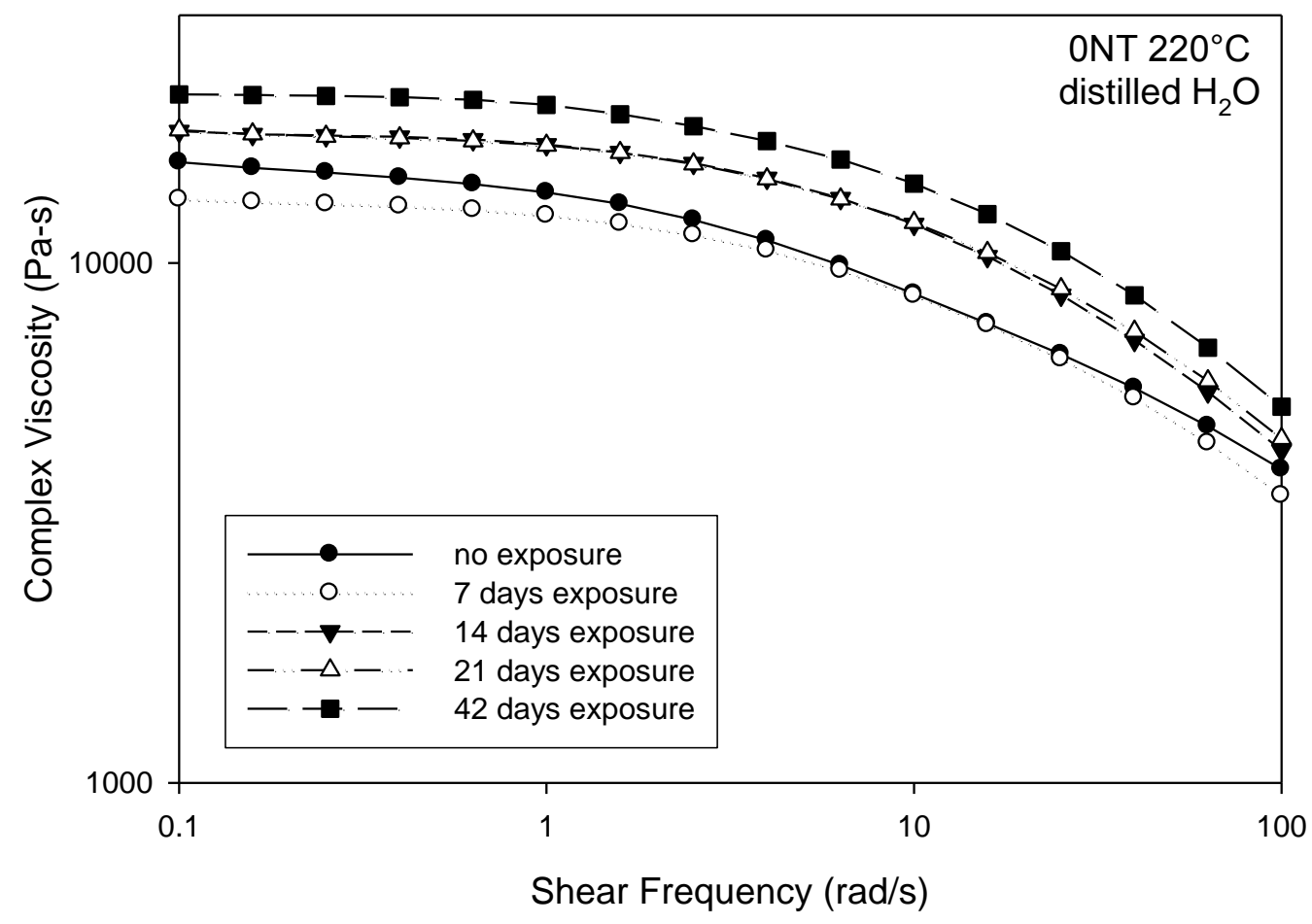

Figure A 15: Viscosity change of sample 0NT after distilled water degradation.

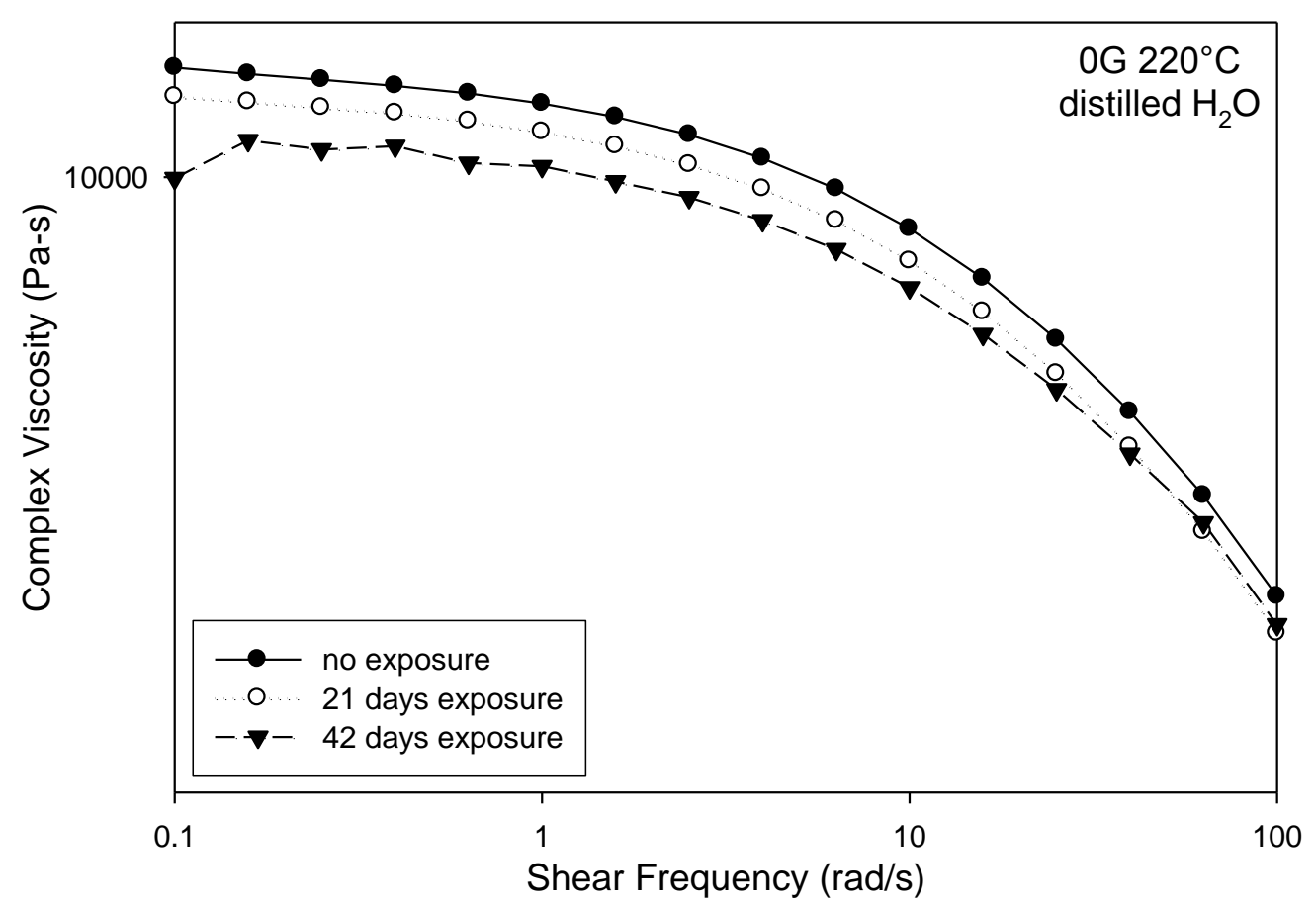

Figure A 16: Viscosity change of sample $0 \mathrm{G}$ after distilled water degradation. 